Portland State University

PDXScholar

Summer 8-2-2018

\title{
3D Hydrodynamic, Temperature, and Water Quality Numerical Model for Surface Waterbodies: Development, Verification, and Field Case Studies
}

Hussein Ali Mahdi Al-Zubaidi

Portland State University

Follow this and additional works at: https://pdxscholar.library.pdx.edu/open_access_etds

Part of the Civil and Environmental Engineering Commons Let us know how access to this document benefits you.

\section{Recommended Citation}

Al-Zubaidi, Hussein Ali Mahdi, "3D Hydrodynamic, Temperature, and Water Quality Numerical Model for Surface Waterbodies: Development, Verification, and Field Case Studies" (2018). Dissertations and Theses. Paper 4500.

https://doi.org/10.15760/etd.6384

This Dissertation is brought to you for free and open access. It has been accepted for inclusion in Dissertations and Theses by an authorized administrator of PDXScholar. Please contact us if we can make this document more accessible: pdxscholar@pdx.edu. 
3D Hydrodynamic, Temperature, and Water Quality Numerical Model for Surface Waterbodies: Development, Verification, and Field Case Studies

by

Hussein Ali Mahdi Al-Zubaidi

A dissertation submitted in partial fulfillment of the requirements for the degree of

Doctor of Philosophy

in

Civil and Environmental Engineering

Dissertation Committee:

Scott A. Wells, Chair

David Jay

Stefan Talke

Raúl Bayoán Cal

Portland State University

2018 
(C) 2018 Hussein Ali Mahdi Al-Zubaidi 


\begin{abstract}
Numerical modeling has become a major tool for managing water quality in surface waterbodies such as rivers, lakes, reservoirs, and estuaries. Since the two-dimensional longitudinal/vertical model CE-QUAL-W2 is a well-known model and it has been applied to thousands of waterbodies around the world successfully, its numerical scheme was adapted to develop a new three-dimensional numerical model for simulating hydrodynamics, temperature, and water quality in surface waterbodies. Finite difference approximations were used to solve the fluid dynamic governing equations of continuity, free water surface, momentums, and mass transport. No coordinate transformations were performed and the $z$-coordinate system has been used. Higher-order schemes (QUICK, QUICKEST, and ULTIMATE QUICKEST) in addition to the UPWIND scheme were used for the advective temperature and mass transport. A novel numerical approach was used for the numerical formulation of the three-dimensional scheme. This approach forced the numerical solution of the free surface equation to be a tri-diagonal matrix form rather than a more computationally intensive penta-diagonal matrix solution. This new approach was done by linking a method called line-by-line with the free water surface numerical solution. Another new approach was that the three-dimensional numerical scheme involved a simultaneous solution of hydrodynamics, temperature, and water quality at every model time level instead of saving the hydrodynamic results to be used later for water quality simulation. Hence, this scheme allowed feedback between the hydrodynamics and water quality every time step. In addition, various unique numerical algorithms were employed from CE-QUAL-W2 such as the W2 turbulence model,
\end{abstract}


selective withdrawal theory, surface heat fluxes, and water quality sources and sinks, making the three-dimensional model built on well-tested algorithms.

To test the model structure and assumptions, an analytical verification was performed by comparing model predictions to known analytical exact solutions test cases. Good agreement was showed by the model for all of these tests. A computation of the volume balance over the simulation period was also incorporated within the model to assess how well the code performed. Sensitivity tests were also made varying bed and wind shear.

The model was also applied to three reservoirs in the USA as field case studies: Lake Chaplain in WA, Laurance Lake in OR, and Cooper Creek Reservoir in OR. The model was validated by comparing the model predictions of water levels, velocities, vertical temperature profiles, and dissolved oxygen with field data. Through these real applications, the numerical predictions of the 3D model showed good agreement with field data based on error statistics. The model results of each field case study were discussed separately. In the Lake Chaplain model application, the study was focused on the importance of the higher-order schemes compared to the first-order UPWIND scheme. The model predictions of temperature were determined by using the UPWIND, QUICK, and QUICKEST scheme and compared with field data. The Error statistics of the model predictions compared to field data were an absolute mean error (AME) of $0.065 \mathrm{~m}$ for the water level predictions and an overall AME of $1.62{ }^{\circ} \mathrm{C}, 1.09{ }^{\circ} \mathrm{C}$, and 1.23 ${ }^{\circ} \mathrm{C}$ for the temperature predictions by using the UPWIND, QUICK, and QUICKEST scheme, respectively. In the Laurance Lake model application, a comparison was performed between the present 3D model and the 2D CE-QUAL-W2. Since the 3D 
model was build based on CE-QUAL-W2, differences between the two models were evaluated. Error statistics between the model predictions of water level and temperature compared to field data showed that both models were in good agreement with field data. However, the 3D model AME $\left(0.30 \mathrm{~m}\right.$ for the water level predictions and $0.48{ }^{\circ} \mathrm{C}$ for the temperature predictions) was higher than the $2 \mathrm{D}$ model $(0.03 \mathrm{~m}$ for the water level predictions and $0.42{ }^{\circ} \mathrm{C}$ for the temperature predictions). Finally, the Cooper Creek Reservoir case study was done to show the model predictions of temperature and dissolved oxygen. In this application, vertical temperature profiles were covered the entire simulation period in order to show how the model transfer heat between stratification and non- stratification conditions. The model showed good agreement with field data $\left(0.12 \mathrm{~m}\right.$ AME for the water level predictions, $1.00{ }^{\circ} \mathrm{C}$ overall $\mathrm{AME}$ for the temperature predictions, and $1.32 \mathrm{~g} / \mathrm{m}^{3}$ overall AME for the dissolved oxygen predictions).

Finally, comparisons were made between CE-QUAL-W2 and the 3D model. The 2D model generally performed better in the tests cases if the model user is unconcerned about lateral impacts. The 3D model is important to use when lateral currents and variation in the lateral dimension are important. 


\section{ACKNOWLEDGMENTS}

I would like to express the deepest appreciation to my advisor Dr. Scott A. Wells who has been aiding and assisting me doing this research project sponsored by the Iraqi Ministry of Higher Education and Scientific Research (MOHESR) / University of Babylon.

Furthermore, none of this would have been possible without the deep love, endless support, and extraordinary encouragement of my family. Many thanks to my mother, my wife, my children (Shadan, Mohammed, and Ali). 


\section{TABLE OF CONTENTS}

ABSTRACT i

ACKNOWLEDGMENTS ................................................................................................ iv

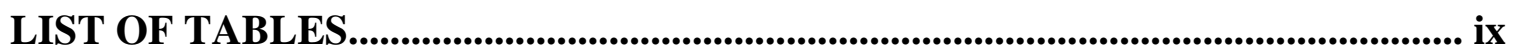

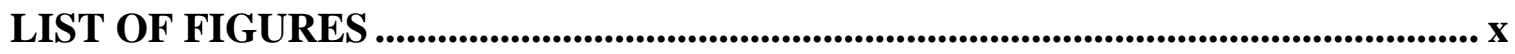

CHAPTER 1. INTRODUCTION ......................................................................................... 1

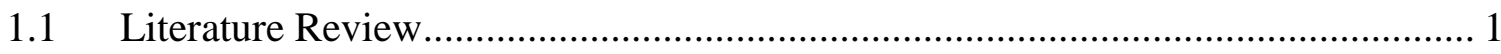

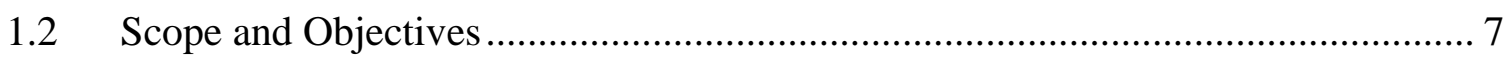

CHAPTER 2. MODEL DESCRIPTION ........................................................................ 10

2.1 Hydrodynamic Governing Equations ……………......................................... 10

2.2 Hydrodynamic Governing Equations Simplifications …………….................... 12

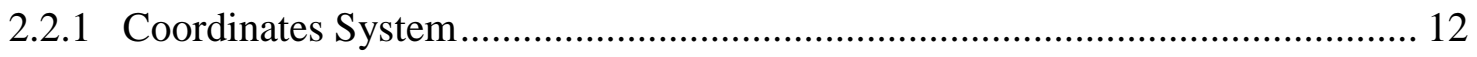

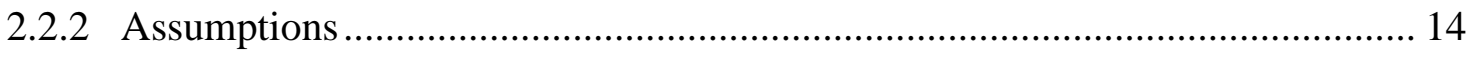

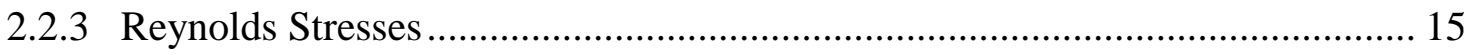

2.2.4 Gravitational Acceleration Components ......................................................... 16

2.2.5 The Hydrostatic Pressure Assumption ........................................................... 17

2.2.6 Lateral Flow Implementation Approach ......................................................... 19

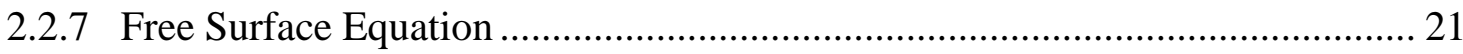

2.3 Heat and Water Quality Transport Governing Equation ..................................... 22

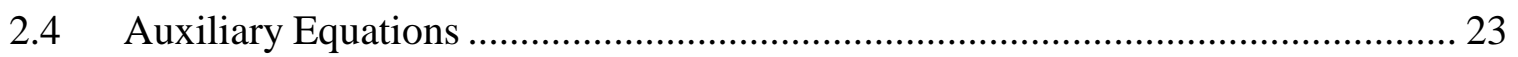

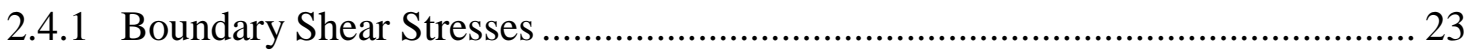

2.4.2 Horizontal Turbulent Eddy Viscosity and Diffusivity …………...................... 27

2.4.3 Vertical Turbulent Eddy Viscosity and Diffusivity ......................................... 28 


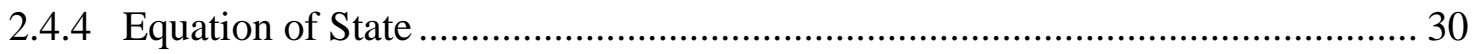

$2.5 \quad$ Numerical Solution Scheme …………………............................................. 33

2.5.1 Computational Grid, Physical Domain, and the Input Bathymetry ................... 33

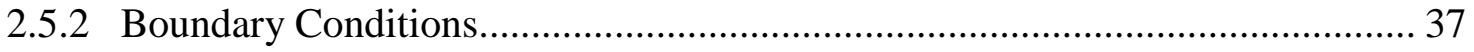

2.5.3 Numerical Solution of the Free Surface Equation ........................................... 42

2.5.4 Numerical Solution of the Momentum Equations........................................... 50

2.5.5 Model Implementation of the Semi-Implicit Finite Differences Scheme .......... 57

2.5.6 Numerical Solution of the Continuity Equation................................................ 65

2.5.7 Numerical Treatment of the Total Waterbody Height ...................................... 66

2.5.8 Numerical Solution of Heat and Water Quality Transport Equation ................. 66

2.5.9 Numerical Solution of the Linear Algebraic Equations ................................... 76

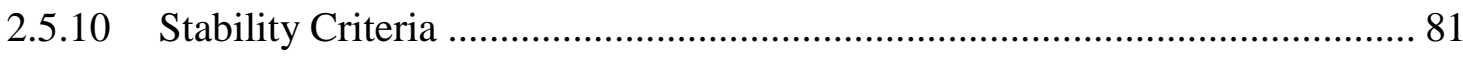

2.5.11 Model Procedure and Programming ........................................................... 82

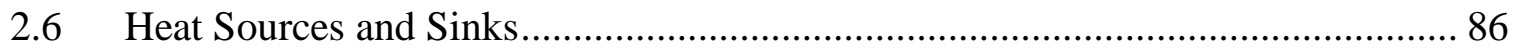

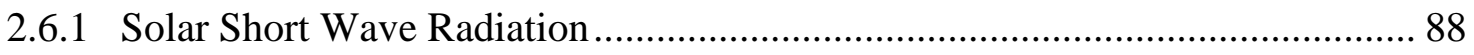

2.6.2 Atmospheric Long Wave Radiation .............................................................. 89

2.6.3 Back (Long Wave) Radiation from the Water Surface ...................................... 90

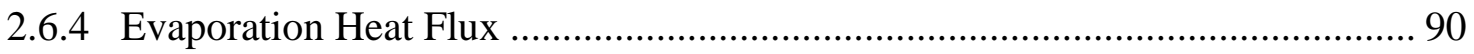

2.6.5 Conduction Heat Flux ……….................................................................. 93

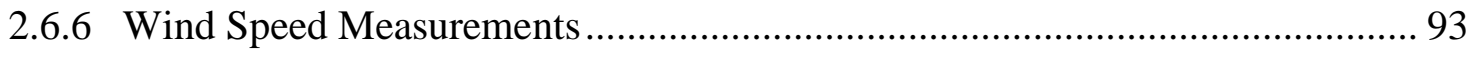

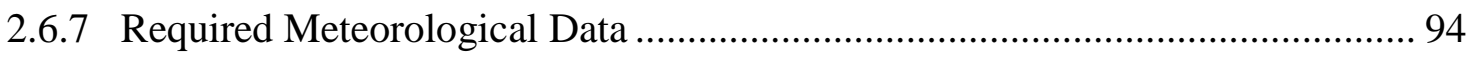

CHAPTER 3. MODEL VERIFICATION.......................................................................... 96

3.1 Test 1: Free Surface Seiching in a Closed Rectangular Basin ............................... 96 
3.2 Test 2: Free Water Surface Response to Wind-Induced Flow in a Closed

Rectangular Basin

3.3 Test 3: Velocity Profile Response to the Wind Induced Flow in a Closed

Rectangular Basin 114

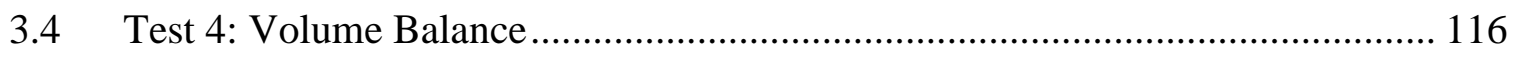

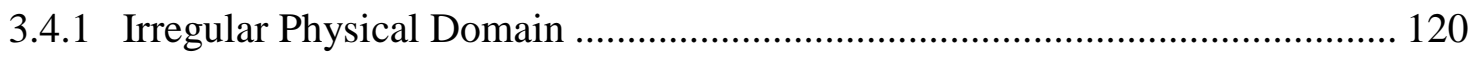

3.4.2 Rectangular Physical Domain .................................................................. 126

3.5 Test 5: Model Sensitivity to the Bottom Resistance............................................ 130

3.6 Test 6: Model Sensitivity to the Wind Induced Flow ......................................... 132

3.7 Test 7: Wetting/Drying Boundary Conditions .................................................... 134

CHAPTER 4. RESULTS AND DISCUSSION: FIELD CASE STUDIES .............. 141

4.1 Case Study 1: Lake Chaplain ...................................................................... 142

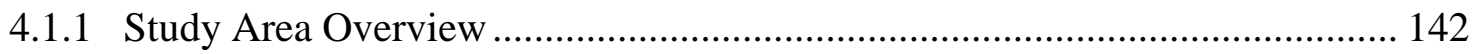

4.1.2 Input Bathymetry and Model Grid Development: ........................................ 144

4.1.3 Inflow/Outflow Boundary Conditions and Meteorological Data..................... 147

4.1.4 Lake Chaplain Model Calibration Results ................................................... 154

4.2 Case Study 2: Laurance Lake - Comparison between the 2D and 3D Model .... 174

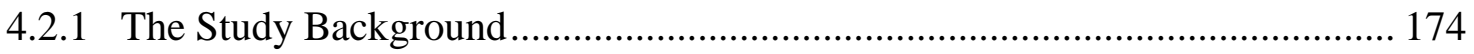

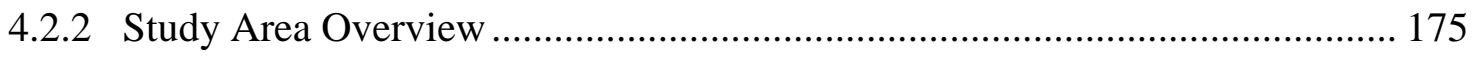

4.2.3 The 3D Model Input Bathymetry and the Grid Development ……………..... 177

4.2.4 The 2D CE-QUAL-W2 Model Grid Setup against the 3D Model Setup......... 178

4.2.5 Inflow/Outflow Boundary Conditions and Meteorological Data..................... 180

4.2.6 Comparison between the 2D and 3D Model for the Lake System.................. 187 vii 
4.2.7 Link between the Hydrodynamics and Water Quality ................................. 198

4.3 Case Study 3: Cooper Creek Reservoir ........................................................... 204

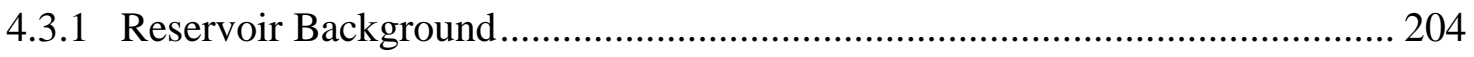

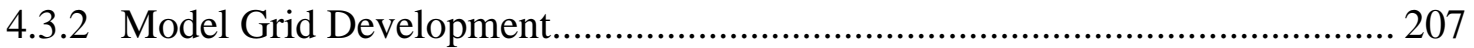

4.3.3 Flow Boundary Conditions and Meteorological Data.................................. 209

4.3.4 Dissolved Oxygen Source/Sink Computations ....................................... 214

4.3.5 Temperature Rate Multipliers ................................................................. 216

4.3.6 Oxygen Limit Computation ............................................................ 218

4.3.7 Cooper Creek Reservoir Model Calibration................................................ 219

CHAPTER 5. CONCLUSIONS AND FUTURE STUDIES .............................. 231

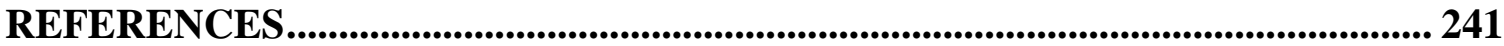




\section{LIST OF TABLES}

Table 1. Overall AME in ${ }^{\circ} \mathrm{C}$ based on $(7,8$, or, 9) comparisons for each temperature profile.

Table 2. Overall AME of CE-QUAL-W2 numerical predictions of Lake Chaplain...... 172

Table 3. Cooper Creek Reservoir dam withdrawals .............................................. 211

Table 4. Overall AME of CE-QUAL-W2 numerical predictions of Cooper Creek

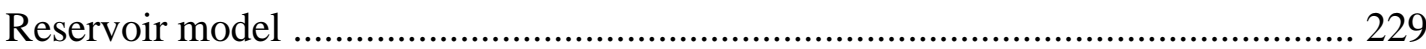

Table 5. Advantages and disadvantages of using the 2D model compared to the 3D

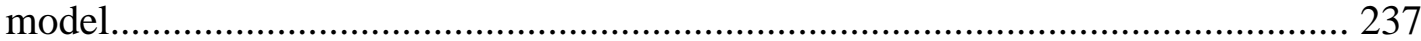

Table 6 . The shape factor corresponding to each case study.................................. 238 


\section{LIST OF FIGURES}

Figure 2-1. Positive direction coordinate system......................................................... 12

Figure 2-2. Shear stresses locations in the control volume........................................... 13

Figure 2-3. Lateral flow implementation in CE-QUAL-W2 model ................................. 20

Figure 2-4. Integration limits of the free surface equation ........................................... 22

Figure 2-5. Wind velocity distribution above the free water surface ............................. 25

Figure 2-6. Variables distribution in a cell ................................................................... 35

Figure 2-7. An example of the computational grid, physical domain, and input

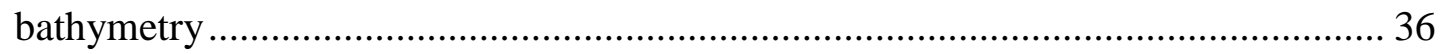

Figure 2-8. Boundary active cells in a waterbody ........................................................ 41

Figure 2-9. Comparison in the water depth between the sparse matrix direct solver

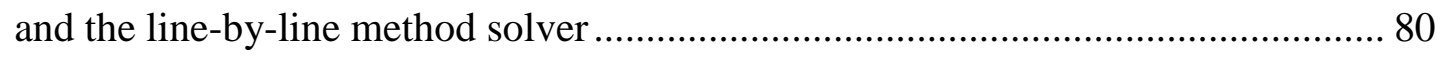

Figure 2-10. Comparison in the water balance generated by the sparse matrix direct

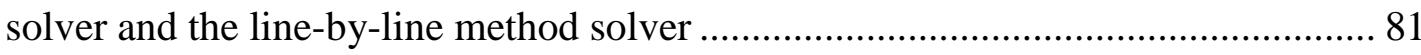

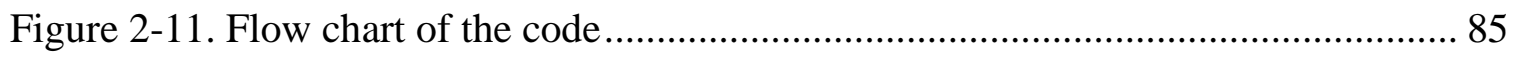

Figure 2-12. Components of the surface heat fluxes ..................................................... 87

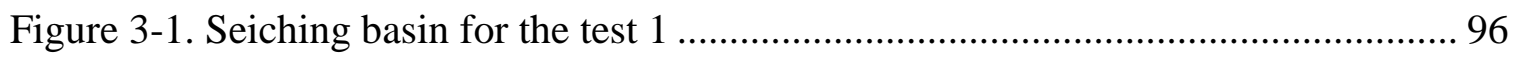

Figure 3-2. Test 1 basin domain and the input bathymetry ........................................ 102

Figure 3-3. Comparison in the water level ( $\eta$ ) between the model results and the analytical solution for the seiching basin near the right boundary $(i=19, j=3$, and $k=k t=3), \Delta \mathrm{t}=50 \mathrm{sec}$. 103 
Figure 3-4. Comparison in the longitudinal velocity $(\mathrm{u})$ between the model results and the analytical solution for the seiching basin near the right boundary $(i=19$, $j=3$, and $k=k t=3), \Delta \mathrm{t}=50 \mathrm{sec}$

Figure 3-5. Comparison in the water level $(\eta)$ between the model results and the analytical solution for the seiching basin near the right boundary $(i=19, j=3$, and $k=k t=3), \Delta \mathrm{t}=5 \mathrm{sec}$

Figure 3-6. Comparison in the longitudinal velocity (u) between the model results and the analytical solution for the seiching basin near the right boundary $(i=19$, $j=3$, and $k=k t=3), \Delta \mathrm{t}=5 \mathrm{sec}$

Figure 3-7. Comparison in the water level $(\eta)$ between the model results and the analytical solution for the seiching basin near the left boundary $(i=3, j=3$, and $k=k t=3), \Delta \mathrm{t}=50 \mathrm{sec}$ 105

Figure 3-8. Comparison in the longitudinal velocity $(\mathrm{u})$ between the model results and the analytical solution for the seiching basin near the left boundary $(i=3$, $j=3$, and $k=k t=3), \Delta \mathrm{t}=50 \mathrm{sec}$ 105

Figure 3-9. Comparison in the water level $(\eta)$ between the model results and the analytical solution for the seiching basin near the left boundary $(i=3, j=3$, and $k=k t=3), \Delta \mathrm{t}=5 \mathrm{sec}$ 106

Figure 3-10. Comparison in the longitudinal velocity (u) between the model results and the analytical solution for the seiching basin near the left boundary $(i=3$, $j=3$, and $k=k t=3), \Delta \mathrm{t}=5 \mathrm{sec}$ 106

Figure 3-11. Dumping effect on the computed water level wave using different time steps for the seiching basin near the right boundary $(i=19, j=3$, and $k=k t=3)$ 107 
Figure 3-12. Dumping effect on the computed water level wave using different time steps for the seiching basin near the right boundary $(i=19, j=3$, and $k=k t=3)$

Figure 3-13. Comparison in water level wave using two spatial resolutions at the same time step 108

Figure 3-14. Effect of the degree of implicitness on damping rate of the computed water level wave for the seiching basin near the left boundary $(i=3, j=3$, and $k=k t=3), \Delta \mathrm{t}=50 \mathrm{sec}$ 108

Figure 3-15. Effect of the degree of implicitness on damping rate of the computed velocity wave for the seiching basin near the left boundary $(i=3, j=3$, and $k=k t=3), \Delta \mathrm{t}=50 \mathrm{sec}$

Figure 3-16. Effect of the degree of implicitness on damping rate of the computed water level wave for the seiching basin near the left boundary $(i=3, j=3$, and $k=k t=3), \Delta \mathrm{t}=5 \mathrm{sec}$

Figure 3-17. Effect of the degree of implicitness on damping rate of the computed velocity wave for the seiching basin near the left boundary $(i=3, j=3$, and $k=k t=3), \Delta \mathrm{t}=5 \mathrm{sec}$

Figure 3-18. Seiching basin for the test 2

Figure 3-19. The computed water level under the wind effect using different time steps for the seiching basin near the left boundary $(i=3, j=3$, and $k=k t=3)$

Figure 3-20. The computed water level under the wind effect using different time steps for the seiching basin near the left and right boundaries ( $i=3$ and $i=19$, $\mathrm{j}=3$, and $k=k t=3$ )

Figure 3-21. Test 3 closed rectangular basin 114 xii 
Figure 3-22. The computed and analytical velocity profile under the effect of wind induced flow in the middle of the seiching basin at time $=1000 \mathrm{sec} \ldots \ldots \ldots \ldots \ldots \ldots \ldots . . . . . . .116$

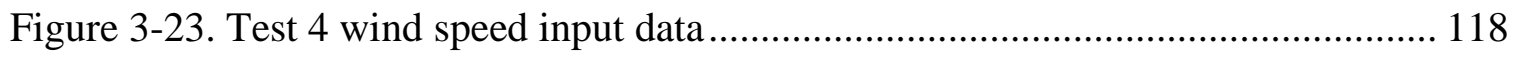

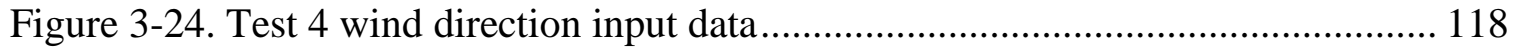

Figure $3-25$. Test 4 air temperature input data.................................................... 119

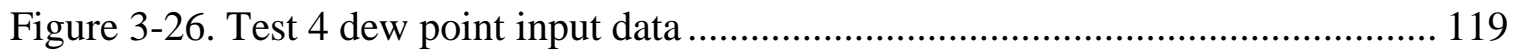

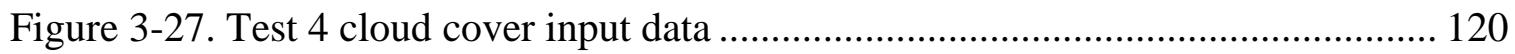

Figure 3-28. Test 4 irregular physical domain and the input bathymetry..................... 122

Figure 3-29. Test 4 surface velocity field for the irregular domain at Julian day $100 \ldots 123$

Figure 3-30. Test 4 volume balance for the irregular domain using $\theta=1$................... 124

Figure 3-31. Test 4 surface layer thickness over time for the irregular domain using

$$
\theta=1
$$

Figure 3-32. Test 4 the volume balance for the irregular domain using $\theta=1$ and $\theta$

$$
=0.5 \text {. }
$$

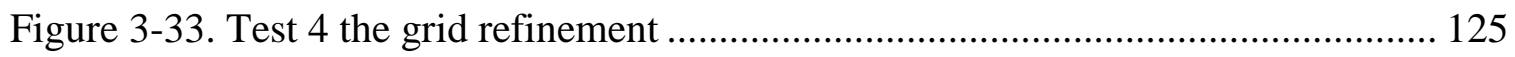

Figure 3-34. Test 4 rectangular physical domain and the input bathymetry ................. 127

Figure 3-35. Test 4 surface velocity field for the rectangular domain at Julian day 100

Figure 3-36. Test 4 volume balance for the rectangular domain using $\theta=1$

Figure 3-37. Test 4 surface layer thickness over time for the rectangular domain

$$
\text { using } \theta=1
$$

Figure 3-38. Test 4 the volume balance for the rectangular domain using $\theta=1$ and $\theta$ $=0.5$ 
Figure 3-39. Test 5 water level sensitivity to the bottom resistance

Figure 3-40 Test 5 vertical velocity profile sensitivity to the bottom resistance

Figure 3-41. Test 6 model sensitivity to the wind induced flow near the left boundary $(i=3, j=3$, and $k=k t=3)$ 133

Figure 3-42. Test 6 volume balance. 133

Figure 3-43. An initial physical domain bathymetry without the step function inflow/outflow boundary conditions 135

Figure 3-44. The step function inflow/outflow boundary conditions during the simulation period 136

Figure 3-45. Drying conditions, Julian day $=1.15$ 137

Figure 3-46. Drying conditions, Julian day $=3.15$ 137

Figure 3-47. Drying conditions, Julian day $=4.15$ 138

Figure 3-48. Wetting conditions, Julian day $=5.15$. 138

Figure 3-49. Wetting conditions, Julian day $=6.15$ 139

Figure 3-50. Wetting conditions, Julian day $=7.15$ 139

Figure 3-51. Wetting conditions, Julian day $=8.15$ 140

Figure 3-52. Wetting conditions, Julian day $=10.15$ 140

Figure 4-1. Lake Chaplain watershed (from Annear et al 2008) 143

Figure 4-2. Lake Chaplain surrounding terrain (Google, 2016) 144

Figure 4-3. Top view of lake Chaplain model grid. 145

Figure 4-4. The model physical domain of Lake Chaplain 146

Figure 4-5. Lake Chaplain inflow 148

Figure 4-6. Lake Chaplain outflow to the paper mill 148 
Figure 4-7. Lake Chaplain outflow to the water treatment plant

Figure 4-8. Lake Chaplain inflow temperature

Figure 4-9. Initial temperature profile and normalized u-velocity at the dam withdrawal

Figure 4-10. Initial temperature profile and normalized v-velocity at the paper mill withdrawal

Figure 4-11. Lake Chaplain wind speed input data 152

Figure 4-12. Lake Chaplain wind direction input data 152

Figure 4-13. Lake Chaplain air temperature input data

Figure 4-14. Lake Chaplain dew point temperature input data

Figure 4-15. Lake Chaplain cloud cover input data.

Figure 4-16. Lake Chaplain model predictions of water surface elevation compared with data using UPWIND scheme and WSC of 0.8

Figure 4-17. Comparison between Lake Chaplain model predictions of velocities with data at $i=11$ and $j=5$ at $6 \mathrm{~m}$ depth.

Figure 4-18. Comparison between Lake Chaplain model predictions of velocities with data at $i=11$ and $j=5$ at $8 \mathrm{~m}$ depth

Figure 4-19. Comparison between Lake Chaplain model predictions of velocities with data at $i=11$ and $j=5$ at $10 \mathrm{~m}$ depth

Figure 4-20. Comparison between Lake Chaplain model predictions of velocities with data at $i=11$ and $j=5$ at $12 \mathrm{~m}$ depth

Figure 4-21. Comparison between Lake Chaplain model predictions of velocities with data at $i=11$ and $j=5$ at $12 \mathrm{~m}$ depth.... 158 
Figure 4-22. Lake Chaplain model predictions of temperature vertical profile compared with data using UPWIND scheme and WSC of 0.85 for (Julian day $\leq 250$ ) and 1.00 for (Julian day $>250)$.

Figure 4-23. Lake Chaplain model predictions of temperature vertical profile compared with data using UPWIND scheme and WSC of 0.7 for $(228 \leq$ Julian day $\leq 258$ ) and 0.8 for any time else 164

Figure 4-24. Lake Chaplain model predictions of temperature vertical profile compared with data using QUICK scheme and WSC of 0.85 for (Julian day $\leq$ 250) and 1.00 for (Julian day > 250) 166

Figure 4-25. Lake Chaplain model predictions of temperature vertical profile compared with data using QUICK scheme and WSC of 0.7 for $(228 \leq$ Julian day $\leq 258$ ) and 0.8 for any time else 168

Figure 4-26. Lake Chaplain model predictions of temperature vertical profile compared with data using QUICKEST scheme and WSC of 0.85 for (Julian day $\leq 250)$ and 1.00 for (Julian day $>250)$

Figure 4-27. Lake Chaplain model predictions of temperature vertical profile compared with data using QUICKEST scheme and WSC of 0.7 for $(228 \leq$ Julian day $\leq 258$ ) and 0.8 for any time else 172

Figure 4-28. CE-QUAL-W2 water level numerical predictions of Lake Chaplain model compared to data.

Figure 4-29. CE-QUAL-W2 vertical temperature profiles of Lake Chaplain model compared to data. 173

Figure 4-30. The Laurance Lake aerial view (Berger et al., 2005) 176 
Figure 4-31. The Laurance Lake location (Google, 2017) ....................................... 176

Figure 4-32. Top view of the Laurance Lake model grid ......................................... 178

Figure 4-33. Models bathymetry and the 2D and 3D grid setup .............................. 179

Figure 4-34. Laurance Lake inflow from Clear Branch Creek ................................... 181

Figure 4-35. Laurance Lake inflow from Pinnacle Creek ...................................... 181

Figure 4-36. Laurance Lake inflow temperature of Clear Branch Creek ..................... 182

Figure 4-37. Laurance Lake inflow temperature of Pinnacle Creek............................ 182

Figure 4-38. Laurance Lake outflow to Clear Branch Creek...................................... 183

Figure 4-39. Initial temperature profile and normalized u-velocity at the dam............. 183

Figure 4-40. Wind speed input data of the Laurance Lake model ............................... 184

Figure 4-41. Wind direction input data of the Laurance Lake model........................... 184

Figure 4-42. Air temperature input data of the Laurance Lake model ......................... 185

Figure 4-43. Dew point temperature input data of the Laurance Lake model............... 185

Figure 4-44. Cloud cover input data of the Laurance Lake model .............................. 186

Figure 4-45. Short solar radiation input data of the Laurance Lake model ................... 186

Figure 4-46. The 2D and 3D model predictions of water level compared with field

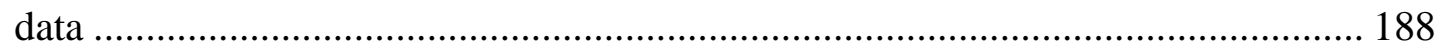

Figure 4-47. Extinction coefficient values based on Secchi disk data......................... 189

Figure 4-48. The 2D model predictions of vertical temperature profiles compared with field data ....................................................................................... 192

Figure 4-49. The 3D model predictions of vertical temperature profiles compared with field data using the QUICKEST scheme 
Figure 4-50. The 3D model predictions of vertical temperature profiles compared with field data using the ULTIMATE QUICKEST scheme 195

Figure 4-51. Surface velocity field of the 3D W3 model 196

Figure 4-52. The 2D and 3D numerical predictions of longitudinal velocities at the lake longitudinal center-line at the end of the simulation time 196

Figure 4-53. Laurance Lake model predictions of vertical ISS profiles in addition to the associated temperature distribution with and without ISS 203

Figure 4-54. Cooper Creek Reservoir location (Google, 2018)................................. 205

Figure 4-55. Cooper Creek Reservoir drainage basin........................................... 206

Figure 4-56. Cooper Creek Reservoir dam outlets ............................................... 207

Figure 4-57. Cooper Creek Reservoir model computational grid................................ 208

Figure 4-58. Creek Reservoir inflow from Cooper Creek ...................................... 210

Figure 4-59. Cooper Creek Reservoir inflow temperature ..................................... 210

Figure 4-60. Air temperature input data of the Cooper Creek Reservoir model ............ 211

Figure 4-61. Dew point temperature input data of the Cooper Creek Reservoir

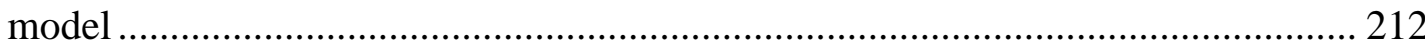

Figure 4-62. Wind speed input data of the Cooper Creek Reservoir model.................. 212

Figure 4-63. Wind direction input data of the Cooper Creek Reservoir model............. 213

Figure 4-64. Cloud cover input data of the Cooper Creek Reservoir model ................. 213

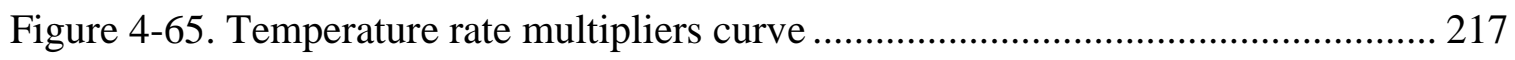

Figure 4-66. Cooper Creek Reservoir model predictions of water surface elevation compared with data using QUICKEST scheme ........................................... 220

Figure 4-67. Cooper Creek Reservoir spillway outflow at the dam ............................ 221 $\mathrm{x}$ viii 
Figure 4-68. Cooper Creek Reservoir model predictions of vertical temperature

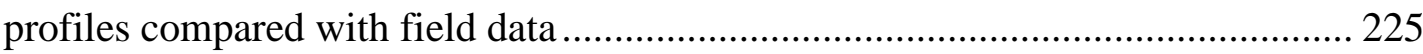

Figure 4-69. Cooper Creek Reservoir model predictions of vertical dissolved oxygen profiles compared with field data ................................................... 228

Figure 4-70. CE-QUAL-W2 water level numerical predictions of Cooper Creek

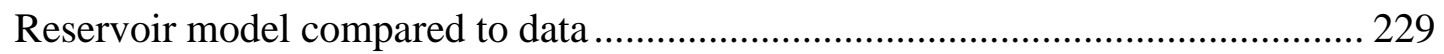

Figure 4-71. CE-QUAL-W2 vertical temperature and dissolved oxygen profiles of Cooper Creek Reservoir model compared to data. 


\section{CHAPTER 1. INTRODUCTION}

\subsection{Literature Review}

Many 3D hydrodynamic and water quality models have been developed since 1960s, and different numerical solution techniques have been used to solve the governing equations depending on the model complexity such as finite differences, finite volume, and finite elements approaches. In this work, we considered the finite differences technique to solve the governing equations. The main restriction in developing any model is the computation time which is related to the solution scheme of the governing equations. Also, each model has been tested either by comparing the predictions with the analytical solution, field data, or both. Thus, each approach has its advantages and disadvantages. Here is a brief description of some well-known three-dimensional models:

Most early group of 3D models used the mode splitting finite difference technique to solve the governing equations. Blumberg and Mellor (1987) developed a threedimensional numerical model for estuarine and coastal ocean circulation. The model was based on the vertical $\sigma$-coordinate and solved the continuity equation, free surface equation, Reynolds momentum equations, and conservation equations for temperature and salinity. The turbulent closure scheme proposed by Mellor and Yamada (1982) was used to obtain the vertical kinematic viscosity and diffusivity, while the horizontal viscosity was calculated according to Smagorinsky (1963). Also, the state equation of Fofonoff (1962) was used to calculate the density from temperature and salinity. The mode splitting finite difference technique based on staggered grids was used to solve the governing equations together with their boundary conditions, wind shear stresses at the 
surface and bottom shear stresses at the bottom. The mode splitting technique separated the governing equations into an external and internal mode. In the external mode, shallow water wave equations were obtained by integrating the governing equations vertically and then solving explicitly in a short time step to satisfy the gravity wave Courant-FriedrichsLevy or CFL limitation. The free surface elevation resulting from the external mode was then used to solve the internal mode, the original governing equations, in a long time step independently from the external mode by treating just the vertical diffusion terms implicitly. This technique helped the stability of the internal mode to not be affected by the gravity wave stability, leading to a much longer time step than the internal mode. Finally, the internal mode produced a tri-diagonal system of linear equations which were then solved by a Gaussian elimination method. Different experiments were done for testing the model performance by making comparisons with field data. This model has been developed by several authors since 1987 when the original model later became the POM, the Princeton Ocean Model (G. L. Mellor, 2002). In the late 1990s and the 2000s, many three-dimensional models have been derived from POM such as ECOM (A F Blumberg \& Mellor, 1987; A. F. Blumberg \& Mellor, 1980), NCOM (Barron, Kara, Martin, Rhodes, \& Smedstad, 2006; Martin et al., 2009), and FVCOM (Chen et al., 2011; Chen, Liu, \& Beardsley, 2003).

Hamrick (1992) developed the Environmental Fluid Dynamic Code, EFDC, which is a three-dimensional model equivalent to Blumberg and Mellor (1987) in its physics and many aspects of the computational scheme. The main differences between the two models are the internal and external mode solution of EFDC model is computed at the 
same model time step. The EFDC model also implements a number of alternate advection transport schemes, such as the central time central space scheme and the forward time upwind space scheme. A further development of EFDC model led to the model EFDCHydro, a special version developed for U.S. EPA Region 4 (Tetra Tech, 2002). The model verification was performed by comparing the numerical solution with field data and calculating the error.

Another group of 3D models is based on the semi-implicit finite difference technique. Casulli and Cheng (1992) developed a three-dimensional numerical model for shallow water flow (TRIM-3D). The governing equations were derived from the Navier-Stokes equations based on turbulent averaging and assuming a constant density and hydrostatic pressure. The non-conservative forms of vertically averaged horizontal and vertical momentum equations, free surface equation, and continuity equation were solved without coordinate transformations in addition to implementing a wetting/drying computational domain. The numerical solution was based on fixed staggered grids with a semi-implicit finite difference method and an Eulerian-Lagrangian method for the convective terms. The vertical diffusion terms were solved implicitly, and the horizontal diffusion terms were solved explicitly. The model stability condition depended on the horizontal viscosity. The numerical solution yielded two types of linear systems: a tri-diagonal matrix from the numerical solution of the horizontal velocities and a penta-diagonal matrix from the numerical solution of the free surface equation. The model was verified and calibrated by implementing it on two different case studies. Also, Casulli and Walters (2000) developed an unstructured grid version of this model, UnTRIM. 
Different models have been developed based on the model of Casulli and Cheng (1992) . The most well-known is ELCOM. Hodges and Dallimore (2006) developed the estuary, lake and coastal ocean model (ELCOM), a three-dimensional model has been used to simulate hydrodynamics and water quality in surface waters. The fundamental numerical scheme was based on the model of Casulli and Cheng (1992) with some adjustments relevant to accuracy, scalar conservation, numerical diffusion, and a new option for calculating vertical turbulent fluxes by application of a mixed-layer turbulence closure scheme. Using the mixed-layer scheme eliminated solving a tri-diagonal matrix for each water column. Whereas the advection terms in hydrodynamic equations were treated similar to TRIM model, a conservative third-order scalar transport method (ULTIMATE QUICKEST) proposed by Leonard (1991) was used.

Ahsan and Blumberg (1999) developed a three-dimensional numerical model for simulating the dynamic and thermal distribution in Onondaga Lake, New York. This model, called ECOMsiz, was another version of Blumberg and Mellor (1987) model, called ECOM. ECOMsiz employed the $z$-coordinate system and used the semi-implicit finite difference method of Casulli and Cheng (1992). Thus, the stability condition depended only on the horizontal viscosity and the solution scheme of the convective terms using an explicit discretization. Moreover, surface heat exchange was included in this work and based on bulk formulas reported in Buchak and Cole (1995). Two years of data, 1985 and 1989, were used to calibrate and validate ECOMsiz.

Furthermore, Edinger (2001) developed the GLLVHT model, the generalized longitudinal lateral vertical hydrodynamic and transport model. This model was 
developed by coupling the momentum equations with the free surface equation to solve for the water level and velocity field. The solution algorithm is similar to TRIM-3D model, but the $z$-coordinate system was employed using constant depth increments in addition to using the Von Karman model for determining the vertical diffusion coefficient of momentum.

Another approach to eliminate the stability related to gravity waves and provide a long time step for a large scale current system economically was illustrated in Bryan (1969) where gravity waves were filtered out of the solution by using a "rigid-lid" approximation. For oceanic circulation, this method may still be adequate, but for lakes and reservoirs under variable wind in space and time, this approach did not reproduce realistic results (P. E. Smith, 2006).

Wang and Falconer (1998) simulated the flow and disinfection processes in disinfection contact tanks by developing a three-dimensional model. Reynolds-averaged equations of continuity and momentums were integrated vertically and then free and bottom boundary conditions and different turbulent closure models were applied. The numerical solution based on a time marching method, an alternating-directions-implicit scheme. In an attempt to remove the numerical diffusion resulting from using the first order accurate upwind scheme, higher order upwind schemes (QUICK and a third order upwind scheme) were used in addition to the first order accurate scheme. This model was validated and investigated by comparing model results with physical model tank results. The mesh consisted of $49 \times 24$ grid with a spatial resolution of $0.043 \mathrm{~m} \times 0.042 \mathrm{~m}$. Various combinations of turbulent closure models and upwind schemes were 
investigated. The results showed that each combination has advantages and disadvantages relevant to chlorine contact tanks.

Thus, the need to develop a new 3D model arises based on the following issues related to water quality models used in practice for reservoir systems:

1) Most $3 \mathrm{D}$ models do not couple water quality and hydrodynamics, hence there is no feedback between hydrodynamics and water quality processes affecting density such as algae growth and suspended solids through effects on the light penetration.

2) The need to an efficient solution scheme for the free surface elevation is existed since this part of the model is the most time consuming part.

3) Most 3D models used for reservoirs do not include selective withdrawal theory (Imberger \& Fischer, 1970). This is an important algorithm to use for dam/reservoir withdrawals since it informs the model as to the vertical layers of the withdrawal without needing to solve the near-field dynamics with the vertical momentum equation.

4) The success and well-tested nature of the 2D CE-QUAL-W2 (Cole \& Wells, 2017) model in accurately simulating the thermal structure and water quality in lakes and reservoirs is a result of algorithms used for turbulence transport, heat transfer, and water quality algorithms that are not found in 3D models. These algorithms have been updated and used in the new 3D model. 
Hence, the present 3D model will solve for water quality transport at the same time step as the hydrodynamic model, use an efficient solution scheme for the free surface elevation resulting in a tri-diagonal matrix form rather than the traditional penta-diagonal matrix form for the solution allowing use of the efficient Thomas algorithm, and use the algorithms found in CE-QUAL-W2 for simulating vertical transport and dam outlet structure interactions.

\subsection{Scope and Objectives}

The objective of this dissertation is to develop a three-dimensional numerical model based on the 2D CE-QUAL-W2 model for simulating hydrodynamic, temperature, and water quality constituents in surface waterbodies. Therefore, this objective was categorized in this dissertation as follows:

- Building the three-dimensional numerical model based on the 2D CE-QUAL-W2 model for simulating hydrodynamic, temperature, and water quality constituents in surface waterbodies (Chapter 2).

- Implementing the semi-implicit scheme in the numerical solution of the free surface equation to account for the degree of implicitness since the 2D CE-QUAL-W2 model solves the free surface equation fully implicitly (Chapter 2).

- Implementing the line-by-line method for the solution of the linear algebraic equations system generated from the numerical solution of the free surface equation. The line-by-line method is a combination of direct and iterative method and has been used with finite volume methods. Unlike using costly iterative methods to solve this system as what has been done in 3D numerical models, the present model employs 
the line-by-line with finite differencing to save time and allow dealing with irregular boundaries (Chapter 2).

- Using higher-order schemes (QUICK, QUICKEST, and ULTIMATE QUICKEST) in the numerical solution of the transport equation and evaluating the effect of these schemes on the vertical temperature profile (Chapter 2).

- Calculating the sources/sinks of heat, dissolved oxygen, and other water quality constituents such as inorganic suspended solids, focusing on the surface heat fluxes and the impact on dissolved oxygen and inorganic suspended solid dynamics (Chapter 2 and 4).

- Verifying the numerical scheme of the present 3D numerical model by doing a series of test cases (Chapter 3):

Test 1: Free surface seiching in a closed rectangular basin.

Test 2: Free water surface response to wind-induced flow in a closed rectangular basin.

$>$ Test 3: Velocity profile response to the wind induced flow in a closed rectangular basin.

$>$ Test 4: Volume balance:

- Irregular physical domain.

- Rectangular physical domain.

> Test 5: Model sensitivity to the bottom resistance.

Test 6: Model sensitivity to the wind induced flow.

$>$ Test 7: Wetting/Drying boundary conditions. 
- Evaluating the effect of the time step and the gravity wave speed on wave damping as a result of this 3D numerical scheme (Chapter 3).

- Validating the model by applying it to different case studies and evaluate the results by doing comparisons with field data (Chapter 4).

Modeling Lake Chaplain, WA USA.

Modeling Laurance Lake, OR USA.

Modeling Cooper Creek Reservoir, OR USA.

- Implementing the 2D based selective withdrawal theory in the 3D case for simulating waterbody outflows at the outlet structures of the dam and at any other location that requires withdraw water from the waterbody. The selective withdrawal theory allows calculating the outflow temperature and water quality constituents based on their vertical variation upstream the dam (Chapter 4).

- Comparing the 3D model predictions with the 2D CE-QUAL-W2 model. Also, recommendations were made on when $2 \mathrm{D}$ versus $3 \mathrm{D}$ models should be used (Chapter 4 and 5). 


\section{CHAPTER 2. MODEL DESCRIPTION}

\subsection{Hydrodynamic Governing Equations}

The three-dimensional hydrodynamic governing equations are derived from the NavierStokes equations. After averaging the Navier-Stokes equations, the Reynolds-averaged equations were obtained (Cushman-Roisin \& Beckers, 2007). For a detailed derivation, see (Batchelor, 1967; Cushman-Roisin \& Beckers, 2007):

The continuity equation is:

$$
\frac{\partial \rho}{\partial t}+\frac{\partial \rho \bar{u}}{\partial x}+\frac{\partial \rho \bar{v}}{\partial y}+\frac{\partial \rho \bar{w}}{\partial z}=0
$$

Where: $\bar{u}, \bar{v}$, and $\bar{w}$ are the time-averaged velocities in the $x, y$, and $z$-directions, respectively, and $\rho$ is the density.

The momentum equations are:

The X-Momentum: $\frac{d \rho \bar{u}}{d t}+\rho f_{*} \bar{w}-\rho f \bar{v}=-\frac{\partial \bar{p}}{\partial x}+\frac{\partial\left(\tau^{x x}+\tau_{x x}\right)}{\partial x}+\frac{\partial\left(\tau^{x y}+\tau_{x y}\right)}{\partial y}+\frac{\partial\left(\tau^{x z}+\tau_{x z}\right)}{\partial z}$

The Y-Momentum: $\frac{d \rho \bar{v}}{d t}+\rho f \bar{u}=-\frac{\bar{p}}{\partial y}+\frac{\partial\left(\tau^{y x}+\tau_{y x}\right)}{\partial x}+\frac{\partial\left(\tau^{y y}+\tau_{y y}\right)}{\partial y}+\frac{\partial\left(\tau^{y z}+\tau_{y z}\right)}{\partial z}$

The Z-Momentum: $\frac{d \rho \bar{w}}{d t}-\rho f_{*} \bar{u}=-\frac{\partial \bar{p}}{\partial z}-\rho g+\frac{\partial\left(\tau^{z x}+\tau_{z x}\right)}{\partial x}+\frac{\partial\left(\tau^{z y}+\tau_{z y}\right)}{\partial y}+\frac{\partial\left(\tau^{z z}+\tau_{z z}\right)}{\partial z}$

The material derivative $\frac{d}{d t}$ includes the time rate of change and the advective terms:

$$
\frac{d}{d t}=\frac{\partial}{\partial t}+\bar{u} \frac{\partial}{\partial x}+\bar{v} \frac{\partial}{\partial y}+\bar{w} \frac{\partial}{\partial z}
$$


Where:

$f=2 \Omega \sin \varphi$, called Coriolis parameter (positive above the equator, zero at the equator, and negative under the equator),

$f_{*}=2 \Omega \cos \varphi$, called reciprocal Coriolis parameter (positive in both hemispheres and approaches to zero at the poles),

$\Omega=$ rotation rate of the earth,

$\varphi=$ the latitude,

$p=$ pressure

$\mathrm{g}=$ gravitational acceleration,

$\tau^{x x}, \tau^{x y}, \tau^{x z}, \tau^{y x}, \tau^{y y}, \tau^{y z}, \tau^{z x}, \tau^{z y}$, and $\tau^{z z}=$ viscous shear stresses, and

$\tau_{x x}, \tau_{x y}, \tau_{x z}, \tau_{y x}, \tau_{y y}, \tau_{y z}, \tau_{z x}, \tau_{z y}$, and $\tau_{z z}=$ turbulent shear stresses or Reynolds stresses. 


\subsection{Hydrodynamic Governing Equations Simplifications}

\subsubsection{Coordinates System}

In this work, the coordinate system was assumed as shown in Figure 2-1. The $x$-axis is at the free water surface, positive to the right in the flow direction, the $y$-axis is also at the free water surface and positive toward us, and $z$-axis is the vertical axis, positive downward. The tangent of the angle $\alpha$ is the slope of the waterbody in case of rivers modeling. This setting makes velocity components positive in the coordinates' positive directions. In addition, the main stream of the waterbody ( $x$-direction) makes an angle $\theta_{2}$ with the north direction.

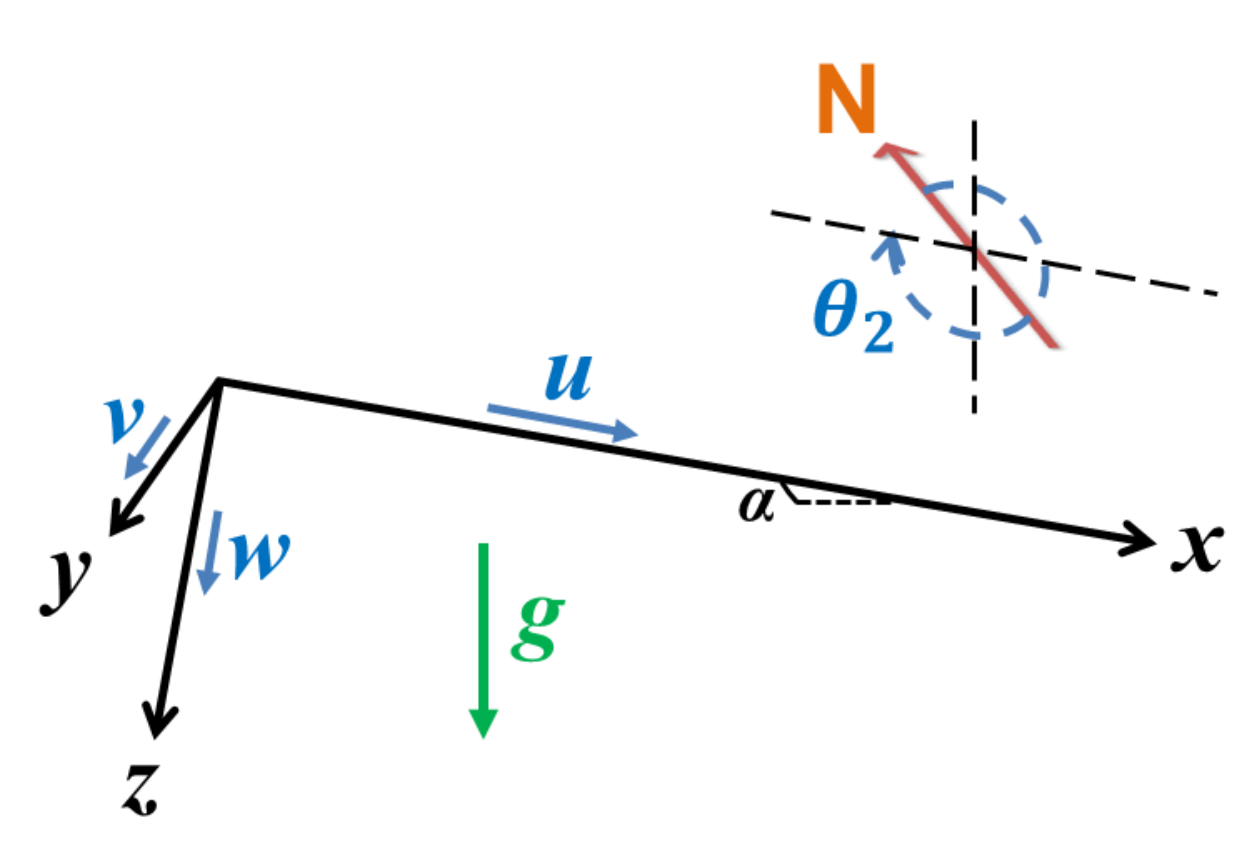

Figure 2-1. Positive direction coordinate system

Based on the present coordinates system, the shear stresses, which act on the control volume faces in $x, y$, and $z$-direction, of the momentum equations are as shown in 

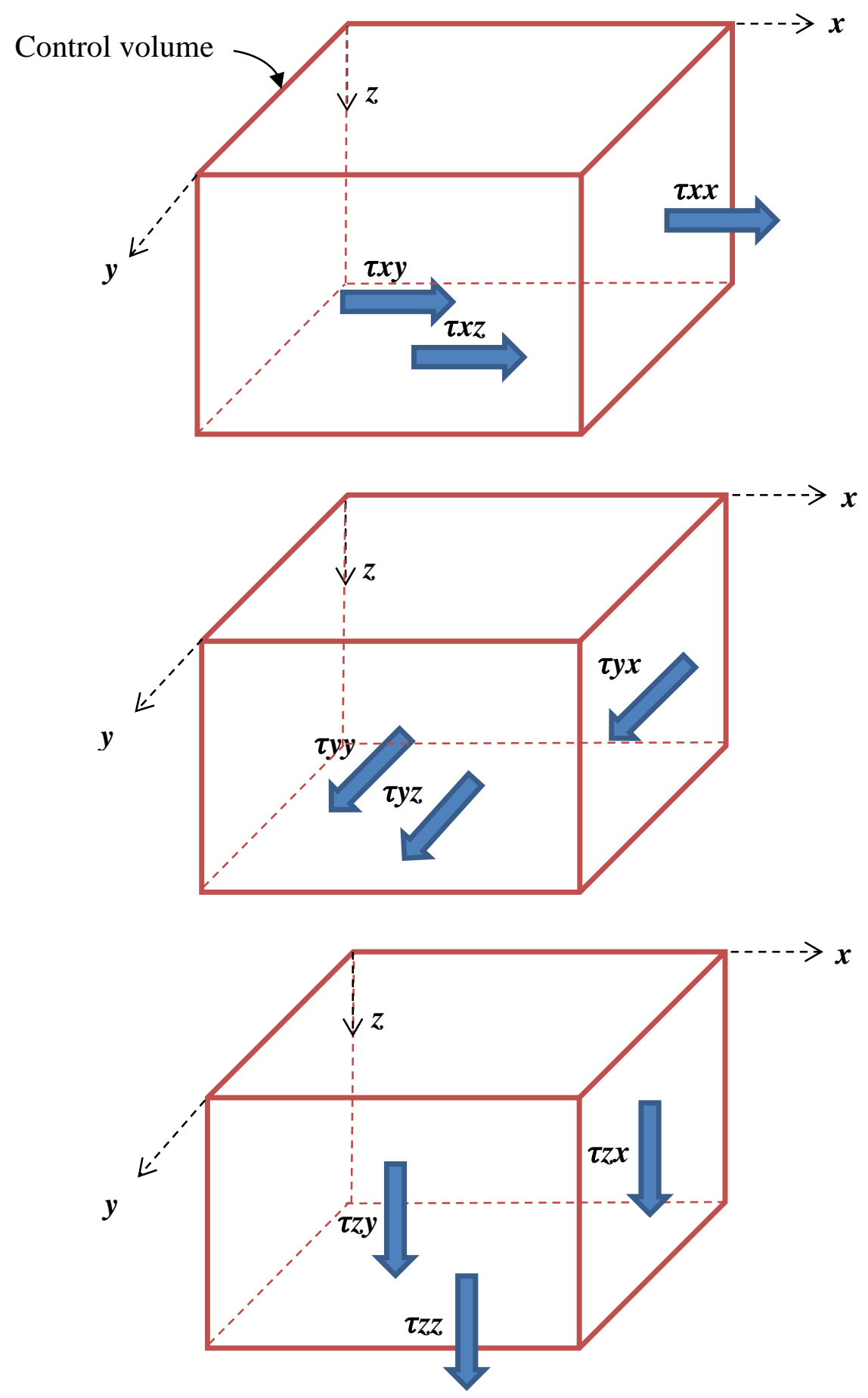

Figure 2-2. Shear stresses locations in the control volume 


\subsubsection{Assumptions}

We will make the following assumptions:

1. The fluid is incompressible.

2. Centripetal acceleration, which is normal to the direction of curved motion toward an instantaneous center of the motion, is used as an adjustment to gravity.

3. Boussinesq approximation of turbulence is valid, and $\rho=\rho_{\circ}+\Delta \rho=\rho_{\circ}$ where $\rho_{\circ}$ is the base density and $\Delta \rho$ represents the variation of $\rho_{\circ}$ with time.

4. The reciprocal Coriolis parameter $\left(f_{*}\right)$ is neglected.

5. The viscous shear stresses are neglected except at the boundaries where the turbulent shear stresses go to zero due to the small amount of energy transferred by the molecular diffusion compared to the turbulent diffusion.

Applying the assumptions to the continuity and momentum equations and rearrange terms, we obtained turbulent time-averaged equations:

The continuity equation:

$$
\frac{\partial \bar{u}}{\partial x}+\frac{\partial \bar{v}}{\partial y}+\frac{\partial \bar{w}}{\partial z}=0
$$

The X-Momentum equation:

$$
\frac{\partial \bar{u}}{\partial t}+\bar{u} \frac{\partial \bar{u}}{\partial x}+\bar{v} \frac{\partial \bar{u}}{\partial y}+\bar{w} \frac{\partial \bar{u}}{\partial z}=-\frac{1}{\rho_{\circ}} \frac{\partial \bar{p}}{\partial x}+f \bar{v}+\frac{1}{\rho_{\circ}} \frac{\partial\left(\tau_{x x}\right)}{\partial x}+\frac{1}{\rho_{\circ}} \frac{\partial\left(\tau_{x y}\right)}{\partial y}+\frac{1}{\rho_{\circ}} \frac{\partial\left(\tau_{x z}\right)}{\partial z}
$$

The Y-Momentum equation:

$$
\frac{\partial \bar{v}}{\partial t}+\bar{u} \frac{\partial \bar{v}}{\partial x}+\bar{v} \frac{\partial \bar{v}}{\partial y}+\bar{w} \frac{\partial \bar{v}}{\partial z}=-\frac{1}{\rho_{\mathrm{o}}} \frac{\partial \bar{p}}{\partial y}-f \bar{u}+\frac{1}{\rho_{\mathrm{o}}} \frac{\partial\left(\tau_{y x}\right)}{\partial x}+\frac{1}{\rho_{\mathrm{o}}} \frac{\partial\left(\tau_{y y}\right)}{\partial y}+\frac{1}{\rho_{\mathrm{o}}} \frac{\partial\left(\tau_{y z}\right)}{\partial z}
$$


The Z-Momentum equation:

$$
\frac{\partial \bar{w}}{\partial t}+\bar{u} \frac{\partial \bar{w}}{\partial x}+\bar{v} \frac{\partial \bar{w}}{\partial y}+\bar{w} \frac{\partial \bar{w}}{\partial z}=-\frac{1}{\rho_{\circ}} \frac{\partial \bar{p}}{\partial z}-\frac{\rho g}{\rho_{\circ}}+\frac{1}{\rho_{\circ}} \frac{\partial\left(\tau_{z x}\right)}{\partial x}+\frac{1}{\rho_{\circ}} \frac{\partial\left(\tau_{z y}\right)}{\partial y}+\frac{1}{\rho_{\circ}} \frac{\partial\left(\tau_{z z}\right)}{\partial z}
$$

\subsubsection{Reynolds Stresses}

Reynolds stresses can be expressed by using the eddy-viscosity concept (Rodi, 1980) in $i$, and $j$ notation form as follows:

$$
\frac{\tau_{i j}}{\rho_{\circ}}=-u_{i}^{\prime} u_{j}^{\prime}=v_{t j} \frac{\partial \bar{u}_{i}}{\partial x_{j}}
$$

Where:

$\bar{u}_{i}=$ the velocity component in the $i$-direction.

$u^{\prime}=$ the fluctuation of the velocity component.

$j=1,2$, and 3 are the coordinate system $x, y$, and $z$, respectively.

$v_{t j}=$ the turbulent kinematic viscosity in the $j$-direction.

This approach is a frequently applied approximation. It is based on assuming the Reynolds stress proportions linearly with the gradient of the time-averaged velocity and in analogous to viscous shear stress.

If we substitute the Reynolds stresses in the momentum equations and use turbulent kinematic viscosities in only the horizontal ( $x$ and $y)$ and vertical $(z)$ directions, the momentum equations in the three directions can be written as follows:

The X-Momentum:

$$
\frac{\partial \bar{u}}{\partial t}+\bar{u} \frac{\partial \bar{u}}{\partial x}+\bar{v} \frac{\partial \bar{u}}{\partial y}+\bar{w} \frac{\partial \bar{u}}{\partial z}=-\frac{1}{\rho_{\circ}} \frac{\partial \bar{p}}{\partial x}+f \bar{v}+\frac{\partial}{\partial x}\left[v_{h} \frac{\partial \bar{u}}{\partial x}\right]+\frac{\partial}{\partial y}\left[v_{h} \frac{\partial \bar{u}}{\partial y}\right]+\frac{\partial}{\partial z}\left[v_{v} \frac{\partial \bar{u}}{\partial z}\right]
$$


The Y-Momentum:

$$
\frac{\partial \bar{v}}{\partial t}+\bar{u} \frac{\partial \bar{v}}{\partial x}+\bar{v} \frac{\partial \bar{v}}{\partial y}+\bar{w} \frac{\partial \bar{v}}{\partial z}=-\frac{1}{\rho_{\circ}} \frac{\partial \bar{p}}{\partial y}-f \bar{u}+\frac{\partial}{\partial x}\left[v_{h} \frac{\partial \bar{v}}{\partial x}\right]+\frac{\partial}{\partial y}\left[v_{h} \frac{\partial \bar{v}}{\partial y}\right]+\frac{\partial}{\partial z}\left[v_{v} \frac{\partial \bar{v}}{\partial z}\right]
$$

The Z-Momentum:

$$
\frac{\partial \bar{w}}{\partial t}+\bar{u} \frac{\partial \bar{w}}{\partial x}+\bar{v} \frac{\partial \bar{w}}{\partial y}+\bar{w} \frac{\partial \bar{w}}{\partial z}=-\frac{1}{\rho_{\circ}} \frac{\partial \bar{p}}{\partial z}-\frac{\rho g}{\rho_{\circ}}+\frac{\partial}{\partial x}\left[v_{h} \frac{\partial \bar{w}}{\partial x}\right]+\frac{\partial}{\partial y}\left[v_{h} \frac{\partial \bar{w}}{\partial y}\right]+\frac{\partial}{\partial z}\left[v_{v} \frac{\partial \bar{w}}{\partial z}\right]
$$

Where: $v_{h}$ is the horizontal turbulent kinematic viscosity, and $v_{v}$ is the vertical turbulent kinematic viscosity.

\subsubsection{Gravitational Acceleration Components}

Assuming there is no change in the bottom elevation, $h$, with $y$-axis $(\partial h / \partial y=0)$, the gravitational acceleration, $\mathrm{g}$, can be resolved into two components. These components increase the momentum in the $x$ and $z$-direction. Thus, one of these components is in the $x$-axis direction, and the other is in the $z$-axis direction.

$$
\begin{aligned}
& g_{x}=-g \frac{\partial h}{\partial x}=g \sin \alpha \\
& g_{z}=-g \frac{\partial h}{\partial z}=g \cos \alpha
\end{aligned}
$$

Adding the two components of the gravitational acceleration to the momentum equations, the equations become:

The X-Momentum:

$$
\frac{\partial \bar{u}}{\partial t}+\bar{u} \frac{\partial \bar{u}}{\partial x}+\bar{v} \frac{\partial \bar{u}}{\partial y}+\bar{w} \frac{\partial \bar{u}}{\partial z}=-\frac{1}{\rho_{\circ}} \frac{\partial \bar{p}}{\partial x}+f \bar{v}+g \sin \alpha+\frac{\partial}{\partial x}\left[v_{h} \frac{\partial \bar{u}}{\partial x}\right]+\frac{\partial}{\partial y}\left[v_{h} \frac{\partial \bar{u}}{\partial y}\right]+\frac{\partial}{\partial z}\left[v_{v} \frac{\partial \bar{u}}{\partial z}\right]
$$


The Y-Momentum:

$$
\frac{\partial \bar{v}}{\partial t}+\bar{u} \frac{\partial \bar{v}}{\partial x}+\bar{v} \frac{\partial \bar{v}}{\partial y}+\bar{w} \frac{\partial \bar{v}}{\partial z}=-\frac{1}{\rho_{\circ}} \frac{\partial \bar{p}}{\partial y}-f \bar{u}+\frac{\partial}{\partial x}\left[v_{h} \frac{\partial \bar{v}}{\partial x}\right]+\frac{\partial}{\partial y}\left[v_{h} \frac{\partial \bar{v}}{\partial y}\right]+\frac{\partial}{\partial z}\left[v_{v} \frac{\partial \bar{v}}{\partial z}\right]
$$

The Z-Momentum:

$$
\frac{\partial \bar{w}}{\partial t}+\bar{u} \frac{\partial \bar{w}}{\partial x}+\bar{v} \frac{\partial \bar{w}}{\partial y}+\bar{w} \frac{\partial \bar{w}}{\partial z}=-\frac{1}{\rho_{\circ}} \frac{\partial \bar{p}}{\partial z}+\frac{\rho g}{\rho_{\circ}} \cos \alpha+\frac{\partial}{\partial x}\left[v_{h} \frac{\partial \bar{w}}{\partial x}\right]+\frac{\partial}{\partial y}\left[v_{h} \frac{\partial \bar{w}}{\partial y}\right]+\frac{\partial}{\partial z}\left[v_{v} \frac{\partial \bar{w}}{\partial z}\right]
$$

\subsubsection{The Hydrostatic Pressure Assumption}

This assumption is also called shallow water assumption. When the horizontal accelerations are larger than the vertical accelerations, a scaling analysis of the zmomentum equation shows that all terms can be cancelled except the first and second term in the right side of the equation because the vertical length scale is much less than the horizontal length scale (Cushman-Roisin \& Beckers, 2007). In other word, the vertical momentum transport is small compared to the horizontal momentum transport; therefore, it can be neglected and we can assume the flow is approximately horizontal (V. Casulli \& Stelling, 1995). Thus, the z-momentum equation becomes:

$$
0=-\frac{1}{\rho_{\circ}} \frac{\hat{\partial p}}{\partial z}+\frac{\rho g}{\rho_{\circ}} \cos \alpha
$$

Solving this first order differential equation leads to the following equation:

$$
\bar{p}=\overline{p_{a}}+g \cos \alpha \int_{\eta}^{z} \rho d z
$$

Where: $\eta$ is the free surface elevation, and $p_{a}$ is the atmospheric pressure at the free surface elevation. 
By taking the derivative of the pressure equation with respect to $x$ and $y$, and applying Leibnitz's rule, we get a new expression for the pressure gradient in $x$ and $y$-direction:

$$
\begin{aligned}
& \frac{\partial \bar{p}}{\partial x}=\frac{\partial \overline{p_{a}}}{\partial x}+g \cos \alpha \int_{\eta}^{z} \frac{\partial \rho}{\partial x} d z-\rho g \cos \alpha \frac{\partial \eta}{\partial x} \\
& \frac{\partial \bar{p}}{\partial y}=\frac{\partial \overline{p_{a}}}{\partial y}+g \cos \alpha \int_{\eta}^{z} \frac{\partial \rho}{\partial y} d z-\rho g \cos \alpha \frac{\partial \eta}{\partial y}
\end{aligned}
$$

Assuming there is no change in the atmospheric pressure $\left.\left(\partial \overline{p_{a}} / \partial x\right)=\left(\partial \overline{p_{a}} / \partial y\right)=0\right)$, and then by substituting the pressure terms in $x$ and $y$-momentum equation, the final governing momentum equations become:

The X-Momentum equation:

$$
\begin{aligned}
\frac{\partial \bar{u}}{\partial t}+\bar{u} \frac{\partial \bar{u}}{\partial x}+\bar{v} \frac{\partial \bar{u}}{\partial y}+\bar{w} \frac{\partial \bar{u}}{\partial z} & =g \cos \alpha \frac{\partial \eta}{\partial x}-\frac{g \cos \alpha}{\rho_{\circ}} \int_{\eta}^{z} \frac{\partial \rho}{\partial x} d z+f \bar{v}+g \sin \alpha \\
& +\frac{\partial}{\partial x}\left[v_{h} \frac{\partial \bar{u}}{\partial x}\right]+\frac{\partial}{\partial y}\left[v_{h} \frac{\partial \bar{u}}{\partial y}\right]+\frac{\partial}{\partial z}\left[v_{v} \frac{\partial \bar{u}}{\partial z}\right]
\end{aligned}
$$

The Y-Momentum equation:

$$
\begin{aligned}
\frac{\partial \bar{v}}{\partial t}+\bar{u} \frac{\partial \bar{v}}{\partial x}+\bar{v} \frac{\partial \bar{v}}{\partial y}+\bar{w} \frac{\partial \bar{v}}{\partial z} & =g \cos \alpha \frac{\partial \eta}{\partial y}-\frac{g \cos \alpha}{\rho_{\circ}} \int_{\eta}^{z} \frac{\partial \rho}{\partial y} d z-f \bar{u} \\
& +\frac{\partial}{\partial x}\left[v_{h} \frac{\partial \bar{v}}{\partial x}\right]+\frac{\partial}{\partial y}\left[v_{h} \frac{\partial \bar{v}}{\partial y}\right]+\frac{\partial}{\partial z}\left[v_{v} \frac{\partial \bar{v}}{\partial z}\right]
\end{aligned}
$$

The hydrostatic pressure equation:

$$
\frac{\hat{\phi}}{\partial z}=\rho g \cos \alpha
$$




\subsubsection{Lateral Flow Implementation Approach}

One implementation approach is the CE-QUAL-W2 approach. Additional momentums from the lateral tributaries cause shear stresses in the longitudinal and lateral direction. These shear stresses can be added to the governing momentum equations. If we assume the tributaries enter the main stream as shown in Figure 2-3, the main velocity of the tributary that enters the main stream can be analyzed into two components:

$$
\begin{aligned}
& u_{x}=u \cos \beta \\
& u_{y}=u \sin \beta
\end{aligned}
$$

Where:

$u_{x}=$ longitudinal velocity component of the tributary in $x$-direction of the main stream.

$u_{y}=$ lateral velocity component of the tributary in $y$-direction of the main stream.

$\beta=$ the angle between the main stream and the tributary as shown in Figure 2-3.

Now, the momentum equations become as follows after adding the two lateral velocities:

The X-Momentum:

$$
\begin{aligned}
\frac{\partial \bar{u}}{\partial t}+\bar{u} \frac{\partial \bar{u}}{\partial x}+\bar{v} \frac{\partial \bar{u}}{\partial y}+\bar{w} \frac{\partial \bar{u}}{\partial z} & =g \cos \alpha \frac{\partial \eta}{\partial x}-\frac{g \cos \alpha}{\rho_{\circ}} \int_{\eta}^{z} \frac{\partial \rho}{\partial x} d z+f \bar{v}+g \sin \alpha \\
& +\frac{\partial}{\partial x}\left[v_{h} \frac{\partial \bar{u}}{\partial x}\right]+\frac{\partial}{\partial y}\left[v_{h} \frac{\partial \bar{u}}{\partial y}\right]+\frac{\partial}{\partial z}\left[v_{v} \frac{\partial \bar{u}}{\partial z}\right]+q u_{x}
\end{aligned}
$$

The Y-Momentum:

$$
\begin{aligned}
\frac{\partial \bar{v}}{\partial t}+\bar{u} \frac{\partial \bar{v}}{\partial x}+\bar{v} \frac{\partial \bar{v}}{\partial y}+\bar{w} \frac{\partial \bar{v}}{\partial z} & =g \cos \alpha \frac{\partial \eta}{\partial y}-\frac{g \cos \alpha}{\rho_{\circ}} \int_{\eta}^{z} \frac{\partial \rho}{\partial y} d z-f \bar{u} \\
& +\frac{\partial}{\partial x}\left[v_{h} \frac{\partial \bar{v}}{\partial x}\right]+\frac{\partial}{\partial y}\left[v_{h} \frac{\partial \bar{v}}{\partial y}\right]+\frac{\partial}{\partial z}\left[v_{v} \frac{\partial \bar{v}}{\partial z}\right]+q u_{y}
\end{aligned}
$$


Where $q$ is the lateral flow per unit volume.

Another implementation approach, used in the present 3D model, to deal with the lateral flows instead of adding the two components $\left(u_{x}\right.$ and $\left.u_{y}\right)$ to the momentum equations is by keeping supplying the lateral flow velocity as a lateral velocity component $(v)$ at the boundary at the intersection face between the lateral flow path and the main stream of the waterbody in which the intersection face is treated as an open boundary. The lateral velocity component could be positive or negative based on its direction weather in the positive or in the negative direction of the $y$-axis.

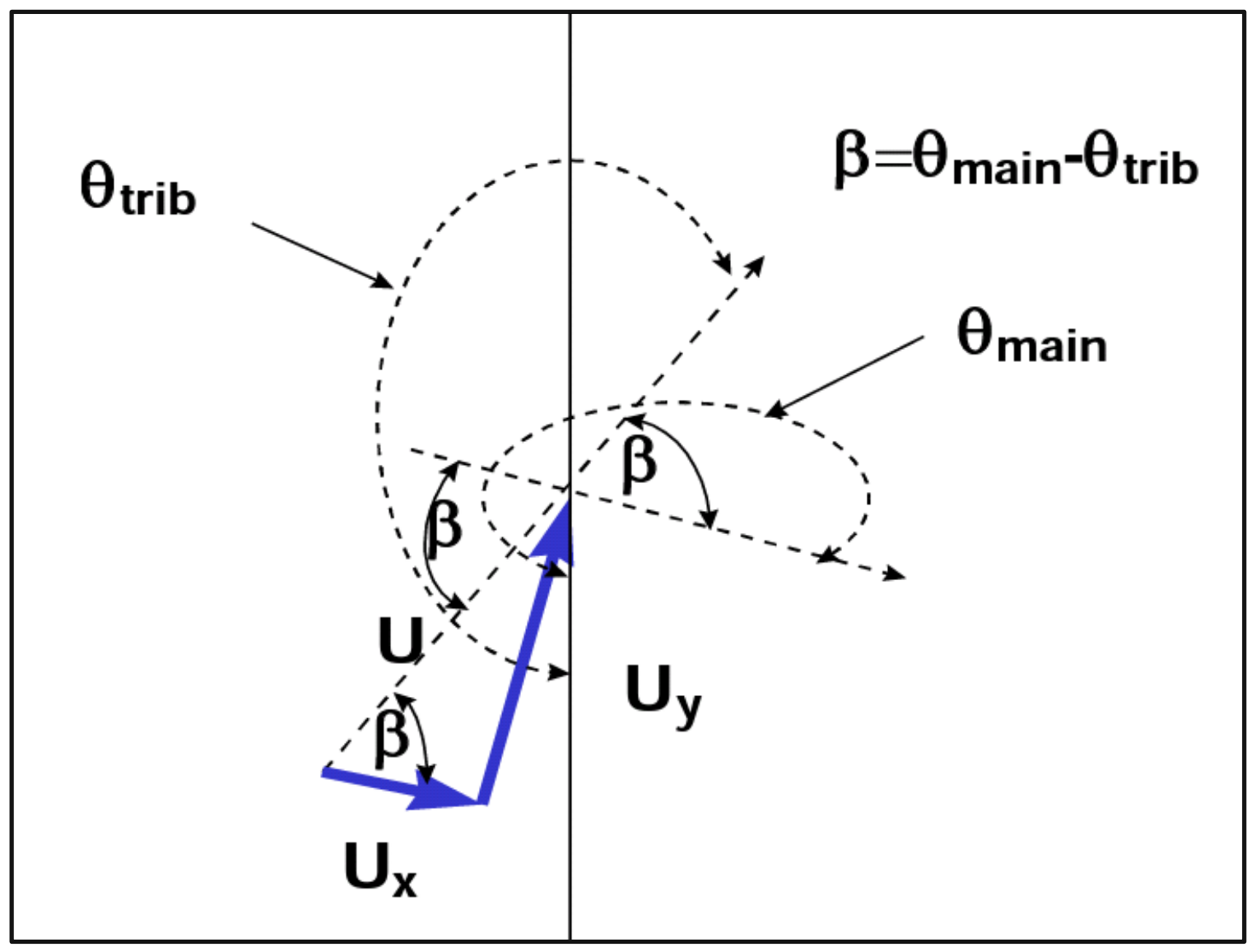

Figure 2-3. Lateral flow implementation in CE-QUAL-W2 model 


\subsubsection{Free Surface Equation}

The free surface equation can be derived by integrating the continuity equation over the total depth (see Figure 2-4 for free surface integration limits) and applying kinematic boundary conditions derived from a mass balance at the surface and bottom layer of the waterbody.

$$
\int_{\eta}^{h} \frac{\partial \bar{u}}{\partial x} d z+\int_{\eta}^{h} \frac{\partial \bar{v}}{\partial y} d z+\int_{\eta}^{h} \frac{\partial \bar{w}}{\partial z} d z=0
$$

Each term is simplified as follows:

$$
\begin{aligned}
& \int_{\eta}^{h} \frac{\partial \bar{u}}{\partial x} d z=\frac{\partial}{\partial x} \int_{\eta}^{h} \bar{u} d z-\frac{\partial h}{\partial x} \bar{u}_{h}+\frac{\partial \eta}{\partial x} \bar{u}_{\eta} \\
& \int_{\eta}^{h} \frac{\partial \bar{v}}{\partial y} d z=\frac{\partial}{\partial y} \int_{\eta}^{h} \bar{v} d z-\frac{\partial h}{\partial y} \bar{v}_{h}+\frac{\partial \eta}{\partial y} \bar{v}_{\eta} \\
& \int_{\eta}^{h} \frac{\partial \bar{w}}{\partial z} d z=\bar{w}_{h}-\bar{w}_{\eta}
\end{aligned}
$$

After substituting the simplified terms in the continuity equation and applying the following boundary conditions:

$$
\begin{array}{ll}
\bar{w}_{h}=\frac{\partial h}{\partial x} \bar{u}_{h}+\frac{\partial h}{\partial y} \bar{v}_{h} & \text { At } z=h \\
\bar{w}_{\eta}=\frac{\partial \eta}{\partial t}+\frac{\partial \eta}{\partial x} \bar{u}_{\eta}+\frac{\partial \eta}{\partial y} \bar{v}_{\eta} & \text { At } z=\eta
\end{array}
$$

Then, the free surface equation is:

$$
\frac{\partial \eta}{\partial t}=\frac{\partial}{\partial x} \int_{\eta}^{h} \bar{u} d z+\frac{\partial}{\partial y} \int_{\eta}^{h} \bar{v} d z
$$




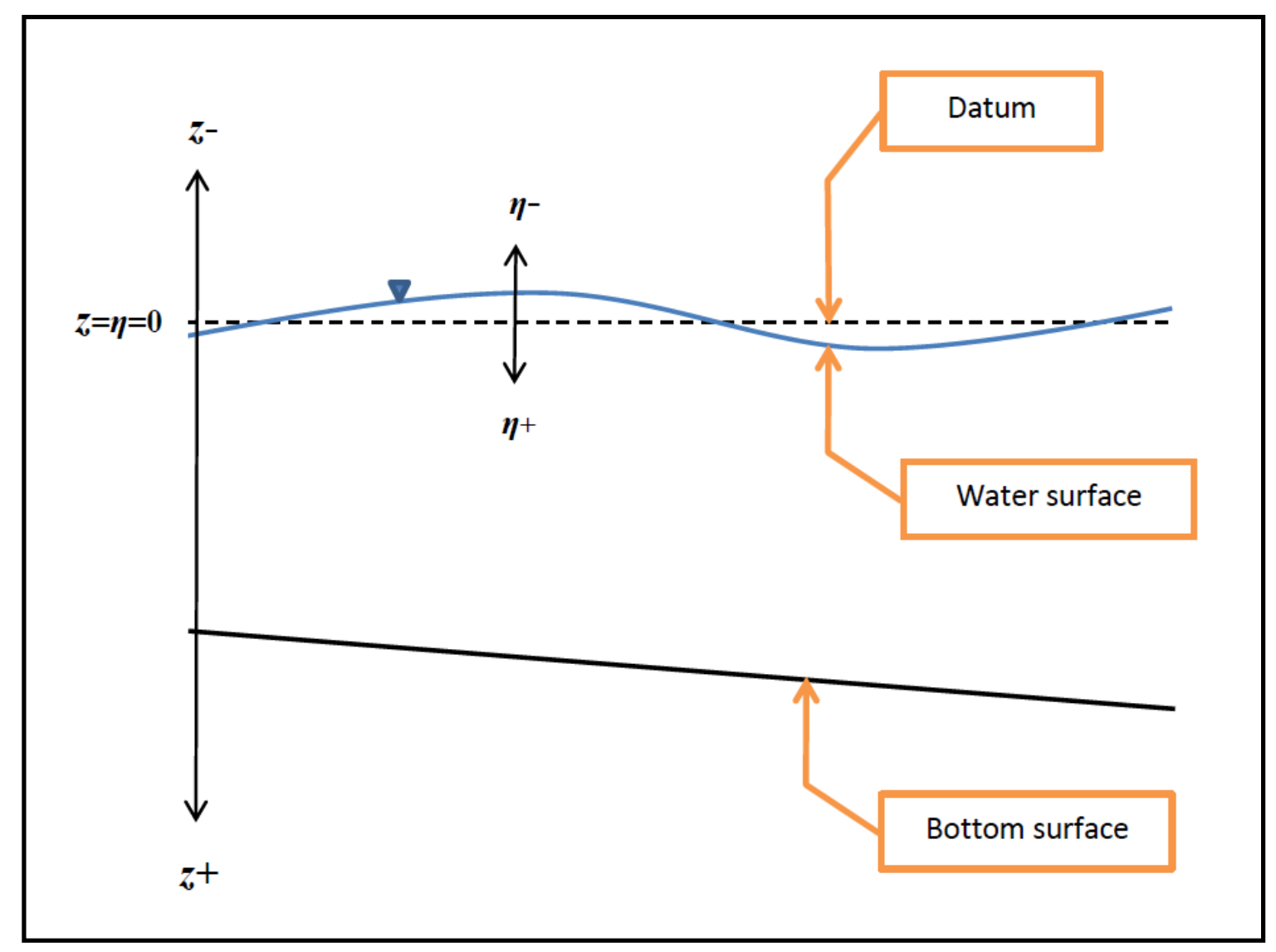

Figure 2-4. Integration limits of the free surface equation

\subsection{Heat and Water Quality Transport Governing Equation}

Heat and water quality transport are governed by the advection diffusion equation which can be written in a general form as follows:

$$
\frac{\partial \phi}{\partial t}+\frac{\partial \bar{u} \phi}{\partial x}+\frac{\partial \bar{v} \phi}{\partial y}+\frac{\partial \bar{w} \phi}{\partial z}=\frac{\partial}{\partial x}\left[D_{x} \frac{\partial \phi}{\partial x}\right]+\frac{\partial}{\partial y}\left[D_{y} \frac{\partial \phi}{\partial y}\right]+\frac{\partial}{\partial z}\left[D_{z} \frac{\partial \phi}{\partial z}\right]+S_{\phi}
$$

Where:

$\phi=$ the constituent concentration $\left(\mathrm{g} / \mathrm{m}^{3}\right)$ 
$S_{\phi}=$ the source/sink term of the constituent $\phi\left(\mathrm{g} / \mathrm{m}^{3} / \mathrm{sec}\right)$, and

$D_{x}, D_{y}$, and $D_{z}=$ the $x, y$, and $z$ diffusion coefficient (diffusivity) $\left(\mathrm{m}^{2} / \mathrm{sec}\right)$, respectively.

Note that, in case of heat transport, $\phi$ becomes the temperature $(T)$ measured in Celsius

$\left({ }^{\circ} \mathrm{C}\right)$ and $S_{\phi}$ measured in a unit of ${ }^{\circ} \mathrm{C} / \mathrm{sec}$. Therefore. $\phi$ is converted to a concentration of

"heat", i.e., using the specific heat of water $\left(\boldsymbol{c}_{\boldsymbol{p}}\right)$ and the water density $(\rho)$,

$$
\phi=\rho c_{p} T
$$

\subsection{Auxiliary Equations}

\subsubsection{Boundary Shear Stresses}

Wind that is blowing above the water surface results in a shear force at the surface acting in the flow direction. Surface shear stresses, or wind shear stresses, are connected to the surface boundary conditions $(z=\eta)$. These stresses are related to the wind velocity distribution above the waterbody, as shown in Figure 2-5, and can be described by using the quadratic drag law (Csanady, 2013; Wu, 1969):

$$
\begin{gathered}
\tau_{s}=\rho_{a} C_{D} U_{w}\left|U_{w}\right| \\
U_{w}=W_{h}-U_{s}
\end{gathered}
$$

Where:

$\tau_{s}=$ the surface shear stress.

$\rho_{a}=$ the air density.

$C_{D}=$ the drag coefficient.

$W_{h}=$ the wind velocity at height $h$, usually is taken at $10 \mathrm{~m}$ height. 
$U_{s}=$ the surface shear velocity.

Because $W_{h} \gg U_{s}, U_{w}$ is assumed equal to $W_{h}$, and the surface shear stresses can be written as follows after analyzing the wind velocity into two components:

$$
\left(\tau_{s x}, \tau_{s y}\right)=\rho_{a} C_{D}\left(W_{x}, W_{y}\right) \sqrt{W_{x}^{2}+W_{y}^{2}}
$$

Where:

$\tau_{s x}$, and $\tau_{s y}=$ the surface shear stresses in the $x$, and $y$-direction, respectively.

$W_{x}$, and $W_{y}=$ the wind velocities in the $x$, and $y$-direction, respectively measured at $10 \mathrm{~m}$ height above the free surface elevation. If the measured wind height is at different height than $10 \mathrm{~m}$, we can calculate the wind velocity at $10 \mathrm{~m}$ height from the following equation (Ryan \& Harleman, 1973):

$$
\frac{W_{Z}}{W_{Z 1}}=\frac{\ln \left(\frac{Z}{Z_{\circ}}\right)}{\ln \left(\frac{Z_{1}}{Z_{\circ}}\right)}
$$

Where:

$W_{z}=$ the wind velocity at elevation $z$.

$W_{Z 1}=$ the wind velocity at elevation $\mathrm{z}_{1}$.

$Z_{\circ}=$ the wind roughness height.

$=0.003 \mathrm{ft}(0.001 \mathrm{~m})$ for wind velocity less than $5 \mathrm{mph}(2.2 \mathrm{~m} / \mathrm{sec})$

$=0.015 \mathrm{ft}(0.0049 \mathrm{~m})$ for wind velocity greater than $5 \mathrm{mph}(2.2 \mathrm{~m} / \mathrm{sec})$ 


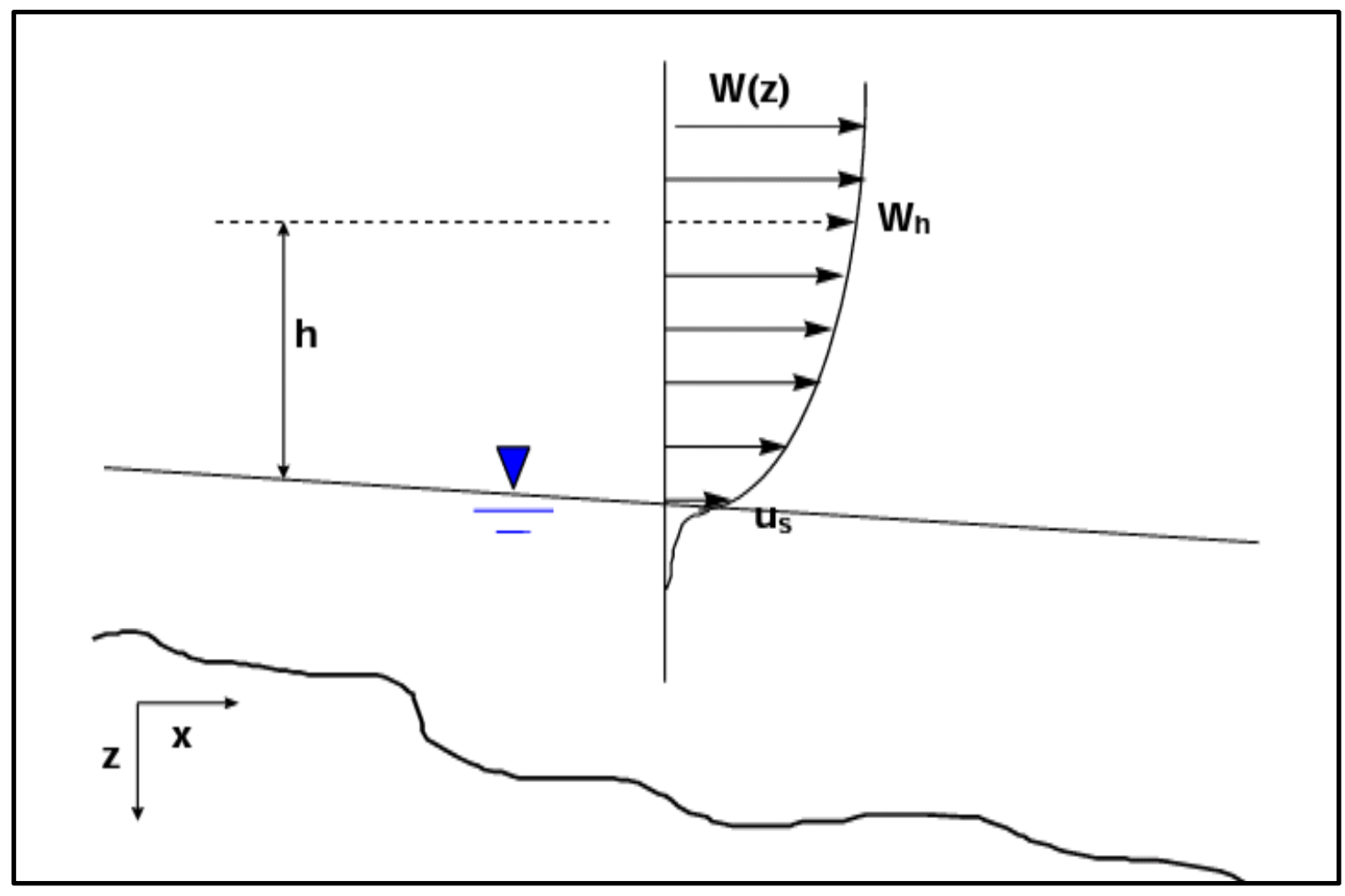

Figure 2-5. Wind velocity distribution above the free water surface

In addition, the wind shear stresses may be written depending on the angle that wind makes with the northern direction:

$$
\left(\tau_{s x}, \tau_{s y}\right)=\rho_{a} C_{D} W_{h}^{2}(\cos \theta, \sin \theta)
$$

Where:

$$
\theta=\theta_{1}-\theta_{2}
$$

$\theta_{1}=$ the angle that wind makes with the northern direction in radians (measured clockwise from the north).

$\theta_{2}=$ the angle that the $x$-direction makes with the northern direction in radians (measured clockwise from the north). 
Bed roughness results in a shear force acting as a resistance force in an opposite direction to the main flow. Bottom shear stresses, or wall shear stresses, are connected to the bottom boundary conditions $(z=h)$ and can also be described by using the quadratic drag law (Dronkers, 1964):

$$
\tau_{b}=\frac{\rho_{w} g}{C^{2}} U|U|
$$

Where:

$\tau_{b}=$ the bottom shear stress.

$U=$ the depth-averaged horizontal velocity.

$\rho_{w}=$ the water density.

$C=$ Chezy's coefficient.

Chezy's coefficient may also be related to the Manning's coefficient,

$$
C=\frac{1}{n} R^{1 / 6}
$$

Where:

$n=$ Manning's coefficient.

$R=$ the hydraulic radius .

In the present three-dimensional model, the bottom shear stresses were calculated depending on the horizontal velocities that are just above the bottom, the bottom layer velocities, by using the quadratic bed stress after analyzing the horizontal velocity into two components $(\bar{u}, \bar{v})$ :

$$
\left(\tau_{b x}, \tau_{b y}\right)=\frac{\rho_{w} g}{C^{2}}(\bar{u}, \bar{v}) \sqrt{\bar{u}^{2}+\bar{v}^{2}}
$$




\subsubsection{Horizontal Turbulent Eddy Viscosity and Diffusivity}

The horizontal turbulent eddy viscosity, $v_{h}$, describes the diffusion of horizontal

momentums. It was assumed a constant value in the present three-dimensional model. A value of $1 \mathrm{~m}^{2} / \mathrm{sec}$ was chosen here as a default value. The user can specify $v_{h}$ based on the case study that the model is developed for. The chosen value of the horizontal turbulent eddy viscosity must not affect the model stability. Thus, a proper value should be investigated during the model calibration in which the model is stable.

The diffusion process of heat and water quality is characterized by the horizontal turbulent eddy diffusivity. Mostly the horizontal turbulent eddy diffusivity is determined experimentally by doing a dye study. One study was conducted by Okubo (1971) in which the apparent diffusivity was expressed as:

$$
D_{a}=\xi \times 10^{-\mathcal{L} \ell}
$$

Where:

$D_{a}=$ the apparent diffusivity, $\mathrm{m}^{2} / \mathrm{sec}$.

$\xi$ and $\mathcal{L}=$ empirical constants.

$\ell=$ the diffusion length scale, $\mathrm{m}$.

Thus, the horizontal turbulent eddy diffusivity in $x$ and $y$-direction $\left(D_{x}\right.$ and $D_{y}$, respectively) can be related to the grid resolution based on Okubo (1971) as follows (Cole \& Wells, 2017; Edinger, 2001):

$$
\begin{aligned}
& D_{x}=5.85 \times 10^{-1.1 \Delta x} \\
& D_{y}=5.85 \times 10^{-1.1 \Delta y}
\end{aligned}
$$




\subsubsection{Vertical Turbulent Eddy Viscosity and Diffusivity}

Eddy viscosity and diffusivity vary significantly in the vertical direction in contrast to the horizontal directions. Therefore, vertical eddy viscosity should be modeled accurately to account for the vertical mixing variability. The present model separates the vertical turbulent kinematic viscosity, $v_{v}$, into two parts (turbulent plus minimum turbulent mixing) in which the turbulent part is adjusted by a stability function:

$$
v_{v}=v_{t}+v_{m}
$$

Where:

$v_{t}=$ the vertical turbulent kinematic viscosity.

$v_{m}=$ the minimum turbulent kinematic viscosity, assumed $\sim 10^{-6} \mathrm{~m}^{2} / \mathrm{sec}$, which is basically the molecular viscosity.

This approach allows a minimum vertical mixing to happen in case of zero turbulent mixing such as at the boundaries where the turbulent shear stresses go to zero.

The vertical turbulent kinematic viscosity under neutral stability conditions, $v_{t o}$, was calculated based on the Von Karman formula (Cole \& Buchak, 1995; Edinger, 2001):

$$
v_{t_{\circ}}=\mathrm{k} \frac{l^{2}}{2} \sqrt{\left[\frac{\partial \bar{u}}{\partial z}\right]^{2}+\left[\frac{\partial \bar{v}}{\partial z}\right]^{2}}
$$

Where:

$\mathrm{k}=$ Von Karman constant, 0.4.

$l=$ the vertical mixing length scale, chosen as a vertical increment $\Delta z$ (Cole \& Buchak, 1995). 
The length scale describes the large eddy size in a turbulent flow. Since the maximum eddy size is the dimension of the problem, it is easy to approximate a reasonable value that gives maximum turbulence energy. Because the model solves the governing equations within a control volume of $(\Delta x \times \Delta y \times \Delta z)$, the vertical length scale that leads to a high vertical turbulent viscosity within the control volume is $\Delta z$. As the mixing length decreases or increases, the amount of mixing intensity or the momentum transfer between the fluid parcels varies based on the Richardson number. Therefore, the vertical turbulent kinematic viscosity under neutral stability conditions needs to be adjusted.

To account for the turbulent mixing intensity due to vertical density stratification, the vertical turbulent mixing is modified by employing the Richardson number criterion for vertical transport of momentum. Different stability functions have been used to adjust the neutral vertical turbulent kinematic viscosity. The following modification proposed by Leendertse and Liu (1975) was adopted:

$$
v_{t}=v_{t_{0}} e^{-1.5 R i}
$$

Where $R i$ is the Richardson number, as high as 10 (Cole \& Buchak, 1995).

For the three-dimensional case, the Richardson number can be written as follows (Sheng \& Butler, 1982):

$$
R i=\frac{g \frac{\partial \rho}{\partial z}}{\rho_{\circ}\left[\left(\frac{\partial \bar{u}}{\partial z}\right)^{2}+\left(\frac{\partial \bar{v}}{\partial z}\right)^{2}\right]}
$$

As a consequence, 
- $R i=0$ neutral stability (no density gradient) conditions in the presence of shear velocity. Therefore, $v_{t}=v_{t_{\mathrm{o}}}$ and stratification does not exist.

- $R i<0$ instability (negative density gradient) conditions. Therefore, $v_{t}>v_{t}$ and there is more turbulent vertical mixing due to buoyancy forces (no stratification).

- $R i>0$ stability (positive density gradient) conditions. Therefore, $v_{t}<v_{t_{0}}$ and there is stratification with a suppression of vertical turbulent mixing. The higher the $R i$ is, the stronger the stratification conditions are.

For the case where the velocity gradient is zero with the presence of density gradient, $R i \rightarrow \infty$, implying that the vertical turbulent eddy viscosity has a value of the molecular viscosity (Ater \& Macdonald, 2013).

The vertical turbulent eddy diffusivity (vertical diffusion coefficient), $D_{v}$, was calculated based on the vertical turbulent kinematic viscosity, $v_{v}$, using the Reynold's analogy (Cole \& Wells, 2017):

$$
D_{v}=0.14 v_{v}
$$

Also and in case of zero turbulent mixing, the vertical diffusivity has a value of the molecular diffusivity $\left(\sim 10^{-6} \mathrm{~m}^{2} / \mathrm{sec}\right)$.

\subsubsection{Equation of State}

The effect of water temperature variation on water density is important, especially during stratified conditions when the water column stability depends on density. Therefore, an accurate density is required for calculating hydrostatic pressure terms of the momentum 
equations. Density variation under the influence of temperature change has been included by applying the equation of state. Additionally, the equation of state links hydrodynamics with the water quality through including the effect of total suspended and dissolved solids on water density. Generally, the water density under the effect of temperature, suspended solid, and dissolved solid can be described as follows (Ford \& Johnson, 1983):

$$
\rho\left(T_{w}, T S S, T D S\right)=\rho_{T_{w}}+\Delta \rho_{T S S}+\Delta \rho_{(T D S \text { or Salinity })}
$$

Where:

$\rho\left(T_{w}, T S S, T D S\right)=$ the water density in $\mathrm{kg} / \mathrm{m}^{3}$ as a function of total suspended solid. (TSS), and total dissolved solid (TDS or Salinity).

$\rho_{T_{w}}=$ the water density in $\mathrm{kg} / \mathrm{m}^{3}$ at a water temperature of $T_{w}$ in ${ }^{\circ} \mathrm{C}$.

$\Delta \rho_{T S S}=$ the water density change in $\mathrm{kg} / \mathrm{m}^{3}$ due to TSS.

$\Delta \rho_{(T D S \text { or Salinity })}=$ the water density change in $\mathrm{kg} / \mathrm{m}^{3}$ due to $T D S$ or Salinity.

Many formulas have been used to write the water density as a function of water temperature. The more precise and the most recommended one is the state equation given by Millero and Poisson (1981) and UNESCO (1981), also restated by Gill (1982) and Martin and McCutcheon (1999):

$$
\begin{gathered}
\rho_{T_{w}}=999.842594+6.793952 \times 10^{-2} T_{w}-9.095290 \times 10^{-3} T_{w}^{2}+1.001685 \\
\times 10^{-4} T_{w}^{3}-1.120083 \times 10^{-6} T_{w}^{4}+6.536332 \times 10^{-9} T_{w}^{5}
\end{gathered}
$$

The effect of total suspended solids, $\Delta \rho_{T S S}$, can be calculated as follows (Gill, 1982):

$$
\Delta \rho_{T S S}=\phi_{T S S}\left[1-\frac{1}{S G}\right] \times 10^{-3}
$$


Where:

$\phi_{T S S}=$ the total suspended solids concentration in $\mathrm{g} / \mathrm{m}^{3}$.

$S G=$ the specific gravity of the suspended solids.

For $S G=2.65$,

$$
\Delta \rho_{T S S}=0.00062 \phi_{T S S}
$$

$\Delta \rho_{\text {sal }}$ can also be calculated as follows (Gill, 1982):

$$
\begin{aligned}
& \Delta \rho_{\text {Sal }}=\left(0.824493-4.0899 \times 10^{-3} T_{w}+7.6438 \times 10^{-5} T_{w}^{2}-8.2467 \times\right. \\
& \left.10^{-7} T_{w}^{3}+\quad 5.3875 \times 10^{-9} T_{w}^{4}\right) \phi_{\text {Sal }}+\left(-5.72466 \times 10^{-3}+1.0277 \times\right. \\
& \left.10^{-4} T_{w}-1.6546 \times \quad 10^{-6} T_{w}^{2}\right) \phi_{\text {Sal }}^{1.5}+4.8314 \times 10^{-4} \phi_{\text {Sal }}^{2}
\end{aligned}
$$

Where $\phi_{\text {Sal }}$ is the salinity in $\mathrm{kg} / \mathrm{m}^{3}$.

If the water is not saline and it has a specified amount of total dissolved solids, the change in density due to total dissolved solids can be determined as follows (Ford \& Johnson, 1983):

$$
\Delta \rho_{T D S}=\left(8.221 \times 10^{-4}-3.87 \times 10^{-7} T_{w}+4.99 \times 10^{-8} T_{w}^{2}\right) \phi_{T D S}
$$

Where $\phi_{T D S}$ is the total dissolved solids in $\mathrm{g} / \mathrm{m}^{3}$. 


\subsection{Numerical Solution Scheme}

\subsubsection{Computational Grid, Physical Domain, and the Input Bathymetry}

An equally spacing staggered grid distribution was used for all variables in the domain as shown in Figure 2-6. The water depth within the computational grid cell is $H$. Each cell was defined at the center by $i, j$, and $k$ in which:

$i=1,2, \ldots \ldots \ldots$, imax-2, imax-1, imax.

$j=1,2, \ldots \ldots \ldots, j \max -2, j \max -1$, jmax.

$k=1,2, \ldots \ldots \ldots, k \max -2, k \max -1, k \max$.

Where the parameters imax, jmax, and kmax are the maximum value of $i, j$, and $k$ in the $x$, $y$, and $z$-direction, respectively.

The domain was divided into computational cells. Some variables other than velocities were defined at the center of the cell, while others were defined at the sides of the cell. The variables $\rho, \phi$, and $H$ are defined at the center $(i, j, k)$, whereas $u, D_{x}, \tau_{x x}$, and $\tau_{y x}$ were defined at $(i+1 / 2, j, k)$ and $(i-1 / 2, j, k)$. The variables $v, D_{y}, \tau_{y y}$, and $\tau_{x y}$ were defined at $(i, j+1 / 2, k)$ and $(i, j-1 / 2, k)$, whereas $w, D_{z}$, and $v_{v}$ were defined at $(i, j, k+$ $1 / 2)$ and $(i, j, k-1 / 2)$. Wherever a variable is required at a location other than its original defined location, linear interpolation based on its surrounding values is used. The variable $\eta$ was taken to be positive downward and negative upward (see Figure 2-4) because $z=0$ was set at the top of the water surface.

An example of the input bathymetry is shown in Figure 2-7. The blue cells hold the water depth in meters at the initial time of simulation, while zero depth cells represent land in 
which there is no water initially. The active cells, where the calculations are done, have a specified water depth, whereas the inactive cells have a zero depth. The inactive cells that are located beside the active cells are used for implementing the boundary conditions. Therefore, inactive cells either have a depth of zero or have a specified depth where the inflow/outflow boundaries exist. The present model assumes the inflow/outflow inactive cells that have a flow in the $x$-direction (in the main stream direction) are located at $i=1$ or $i=$ imax, whereas the inflow/outflow inactive cells that have a flow in the $y$-direction (lateral flow) are located anywhere at the $y$-boundaries that are perpendicular to the $y$ axis. In both cases, the flow that is in the positive axis-direction has a positive velocity component in that direction (see Figure 2-1 for the positive direction of the velocity components).

In addition, we need to define the bottom cell for each water column, $k b(i, j)$. The bottom cells are variable spatially within the physical domain based on the waterbody bathymetry. Therefore, the bottom cell of a water column $(i, j)$ has a maximum $k$ value in that water column in which the bottom cell is wet. Another definition is the top layer, $k t$. The free water surface elevation can be placed initially within the grid at any $z$-level. Then, this location will change with time if the water level went up or down. Thus, the surface cell has a $k$ value of $k t$ during the simulation time, giving:

$$
k t \leq k b(i, j) \leq k \max
$$




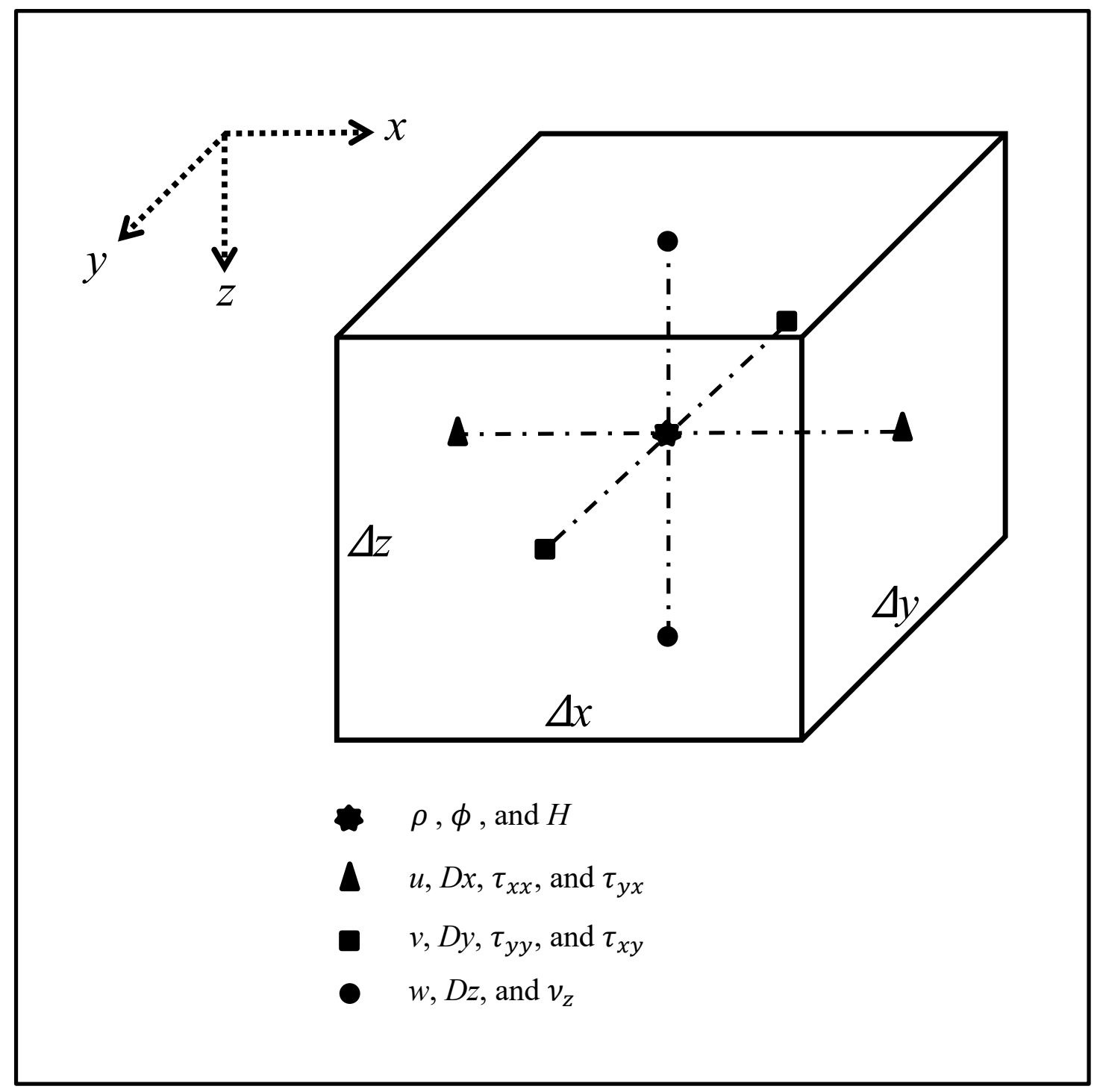

Figure 2-6. Variables distribution in a cell 


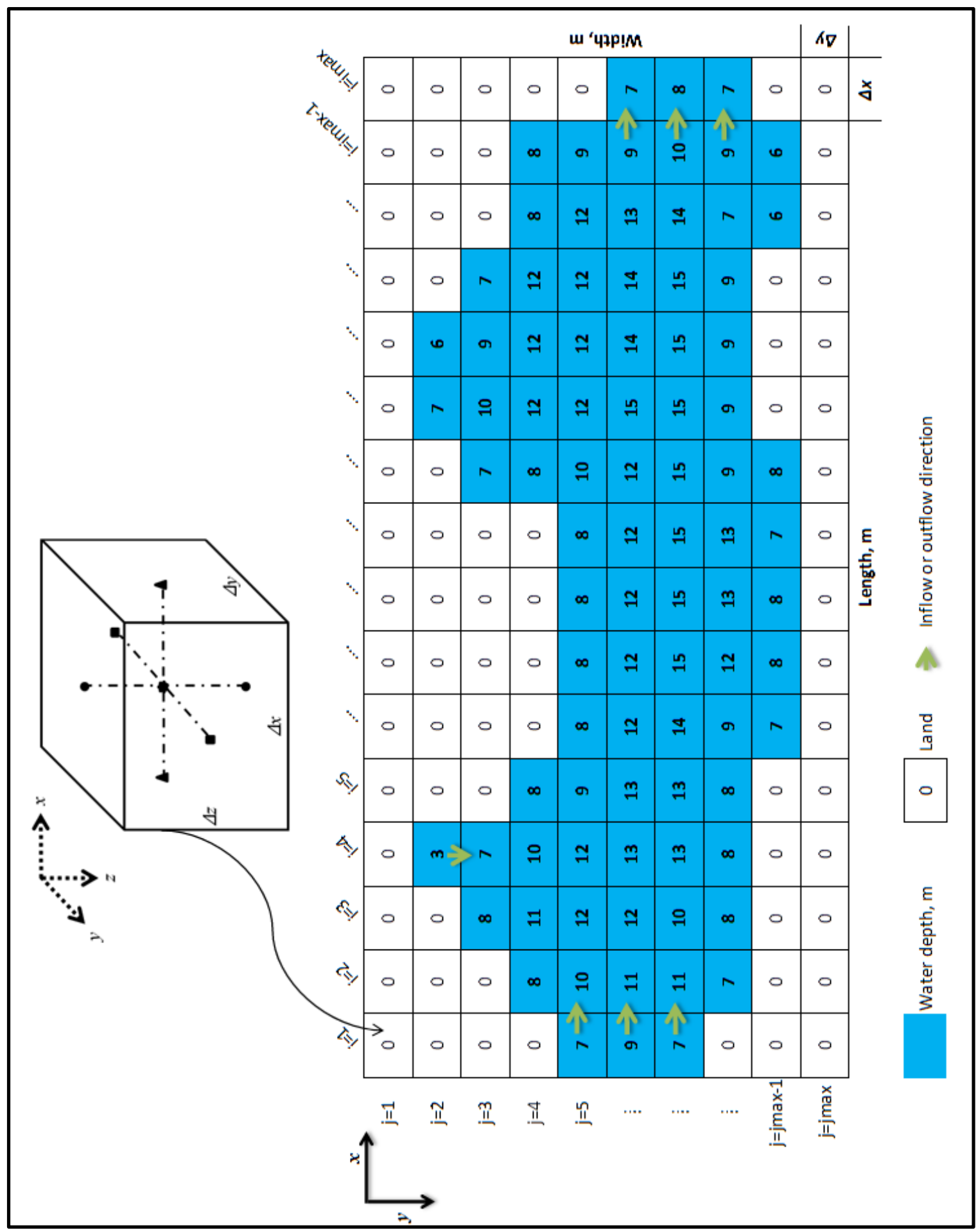

Figure 2-7. An example of the computational grid, physical domain, and input bathymetry 


\subsubsection{Boundary Conditions}

The numerical solution of the governing partial differential equations requires boundary conditions to be specified at the domain boundaries. The number of the boundary conditions needed for each governing equation depends on the highest order spatial derivative of the equation. Since the present three-dimensional model adopted staggered grids, the dependent variables were defined differently at the boundary face depending on the location of that boundary in the domain (west, east, north, and south). Two types of boundaries, either closed or opened, surround the surface waterbodies. Figure 2-8 shows an example of these two boundaries and how the dependent variables were defined at the boundary active cells based on their locations within a waterbody domain of total computational cells $80(5 \times 4 \times 4)$.

The model adopted the following boundary conditions for the hydrodynamic dependent variables:

At the closed boundary faces that are normal to the velocity component direction,

$$
\begin{gathered}
\left.u\right|_{x=\text { closed boundary face }(\text { west or east })}=0 \\
\left.v\right|_{y=\text { closed boundary face }(\text { north or south })}=0 \\
\left.w\right|_{z=\text { bottom }}=0
\end{gathered}
$$

In addition to the following Neumann boundary conditions:

$$
\begin{aligned}
& \left.\frac{\partial \eta}{\partial x}\right|_{x=\text { closed boundary face (west or east) }}=0 \\
& \left.\frac{\partial \eta}{\partial y}\right|_{y=\text { closed boundary face }(\text { north or south) }}=0
\end{aligned}
$$




$$
\frac{\partial u}{\partial z}=\left.\frac{\partial v}{\partial z}\right|_{z=0, z=\text { bottom closed boundary face }}=0
$$

At the opened boundary faces, the model reads the inflow to the domain and the outflow leaving the domain from the input data. These input inflow/outflow data are supplied to the model as time series. The model reads the inflow/outflow at each single time step to determine the equivalent velocity component at the boundary face. Thus, boundary velocities are known at their defined locations and they are functions of time, $u(t)$ and $v(t)$.

$$
\begin{gathered}
\left.u\right|_{x=\text { opened boundary face }(\text { west or east })}=u(t) \\
\left.v\right|_{y=\text { opened boundary face }(\text { north or south })}=v(t)
\end{gathered}
$$

Also, for the free surface elevation, same Neumann boundary conditions that are applied at the close boundaries are used at the opened boundary faces,

$$
\begin{array}{ll}
\left.\frac{\partial \eta}{\partial x}\right|_{x=\text { opened boundary face (west or east) }} & =0 \\
\left.\frac{\partial \eta}{\partial y}\right|_{y=\text { opened boundary face (north or south) }} & =0
\end{array}
$$

For the temperature and constituent transport part of the model, no fluxes exist across the closed boundary faces, giving:

$$
\begin{gathered}
\left.\frac{\partial \phi}{\partial x}\right|_{x=\text { closed boundary face (west or east) }}=0 \\
\left.\frac{\partial \phi}{\partial y}\right|_{y=\text { closed boundary face (north or south) }}=0 \\
\left.\frac{\partial \phi}{\partial z}\right|_{z=\text { bottom closed boundary face }}=0
\end{gathered}
$$


The model uses inactive opened boundary cells to feed the inflow temperature and water quality constituents into the waterbody domain. Therefore, the temperature and the constituent's concentration are known at the inflow inactive opened boundary cells from the input time series data, giving:

$$
\left.\phi\right|_{\text {inflow inactive boundary cells }}=\phi(t)
$$

The outflow temperature and water quality constituents are determined at the outflow inactive cells from the adjacent active cell by setting Neumann boundary conditions for the temperature and the constituent's concentration at the opened boundary faces,

$$
\begin{array}{ll}
\left.\frac{\partial \phi}{\partial x}\right|_{x=\text { opened boundary face (west or east) }} & =0 \\
\left.\frac{\partial \phi}{\partial y}\right|_{y=\text { opened boundary face (north or south) }} & =0
\end{array}
$$

In addition, at the water surface $(z=0)$, the following boundary condition was applied:

$$
\left.\frac{\partial \phi}{\partial z}\right|_{z=0}=0
$$




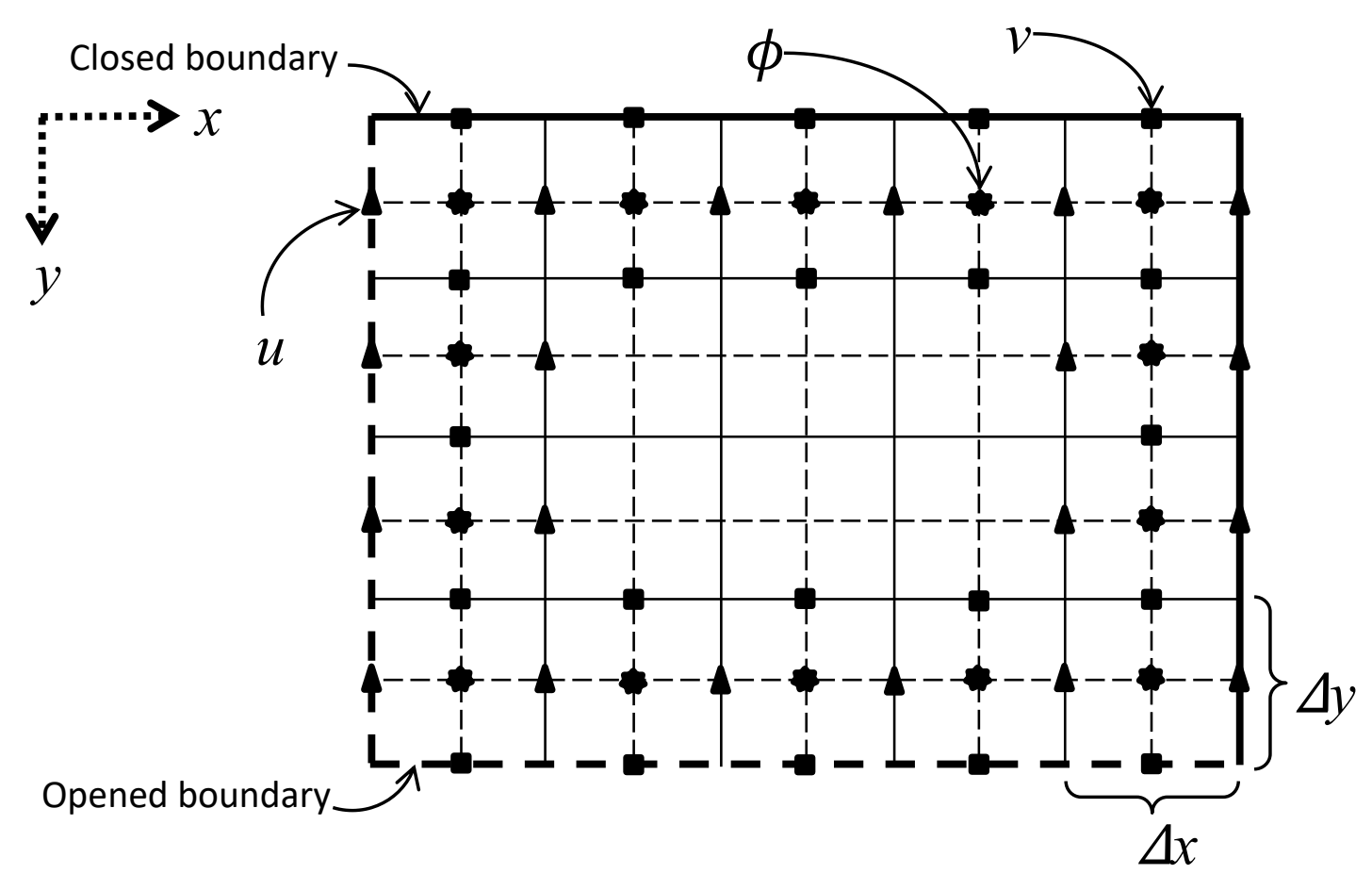

(a) $x-y$ plan view of the waterbody

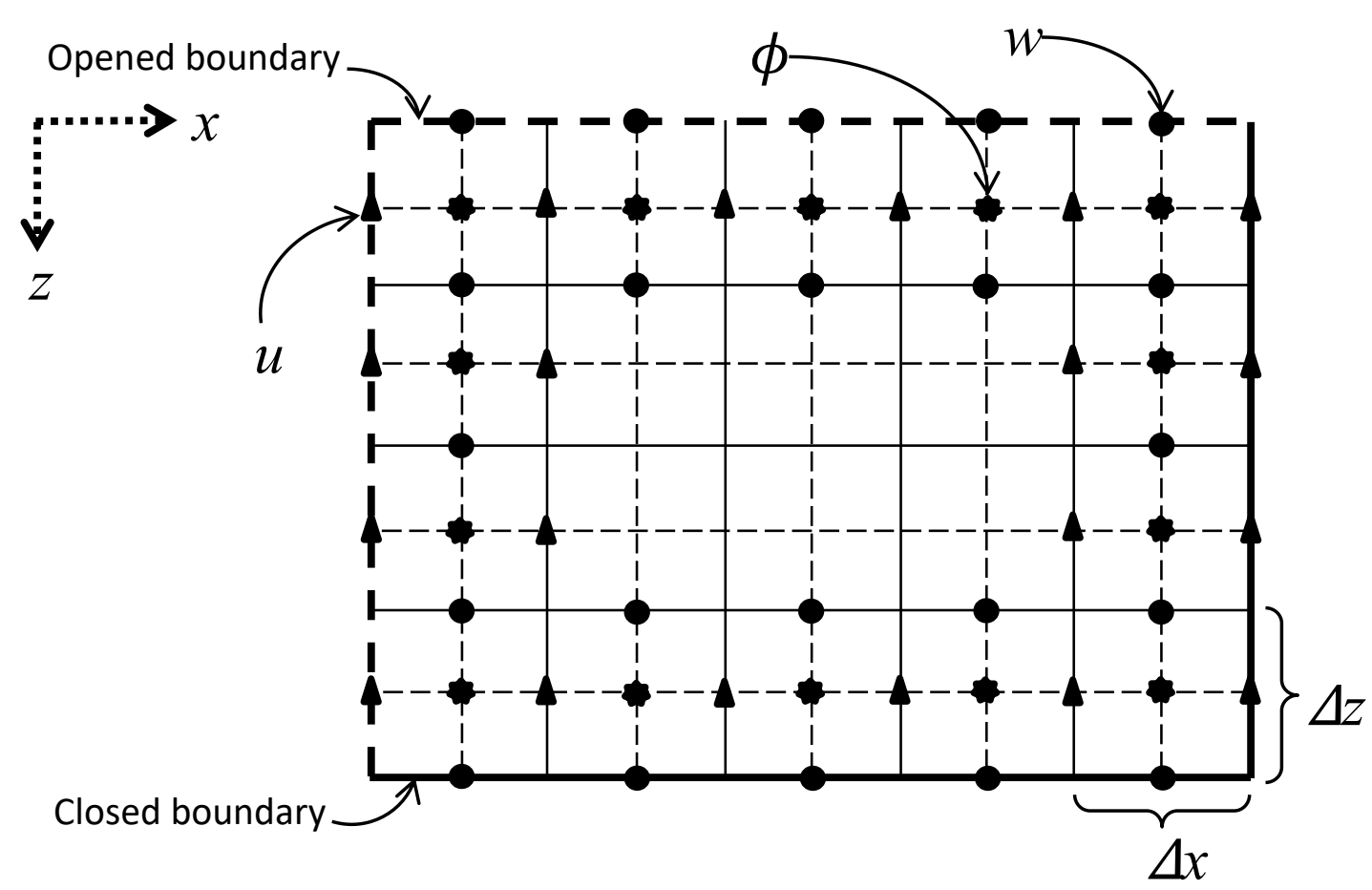

(b) $x-z$ plan view of the waterbody 


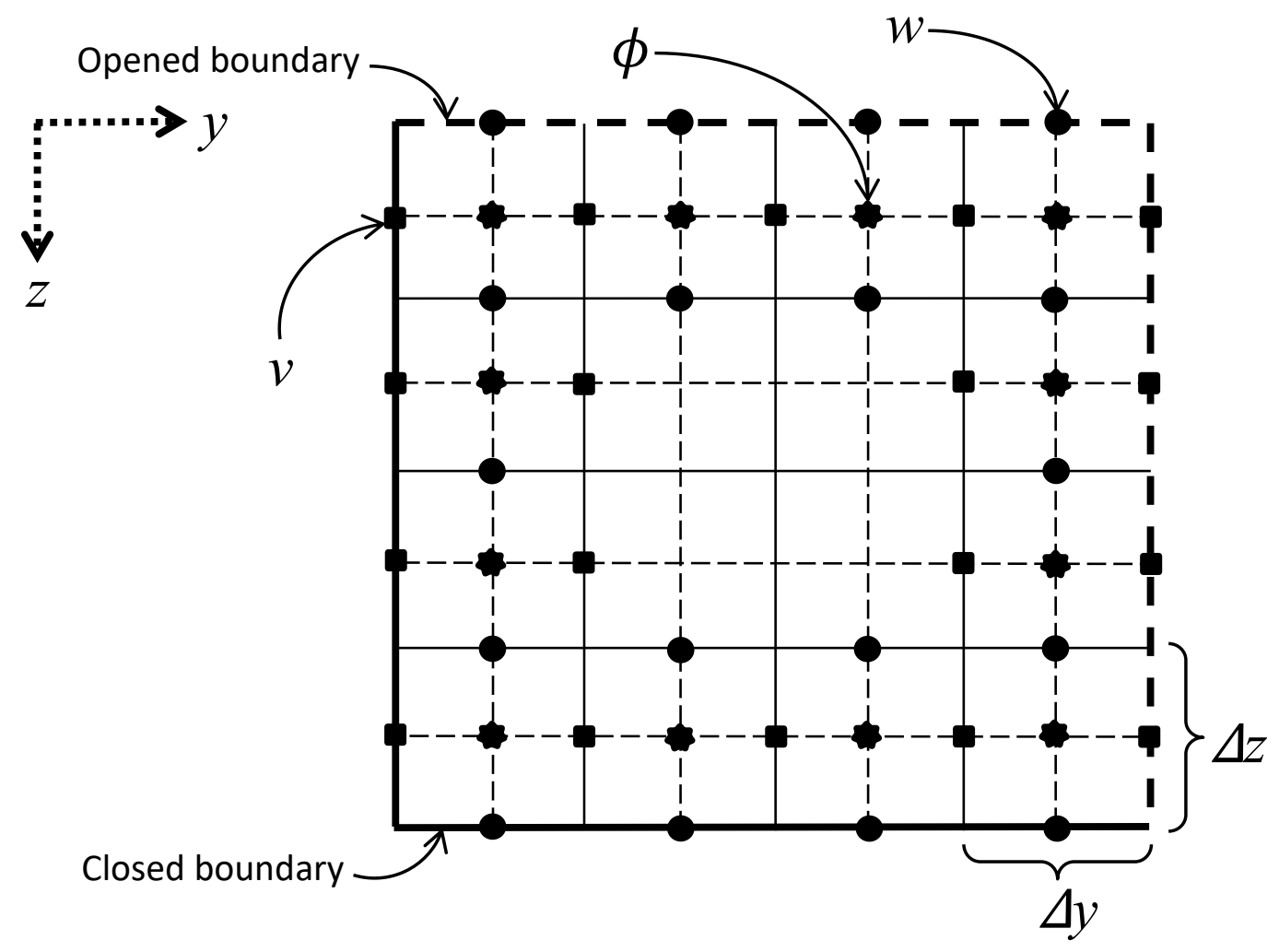

(c) $y-z$ plan view of the waterbody

Figure 2-8. Boundary active cells in a waterbody 


\subsubsection{Numerical Solution of the Free Surface Equation}

The free water surface equation was solved by substituting $\mathrm{X}$ and $\mathrm{Y}$-momentum equation into the free surface equation.

The X-Momentum:

$$
\begin{aligned}
\frac{\partial \bar{u}}{\partial t}+\bar{u} \frac{\partial \bar{u}}{\partial x}+\bar{v} \frac{\partial \bar{u}}{\partial y}+\bar{w} \frac{\partial \bar{u}}{\partial z} & =g \cos \alpha \frac{\partial \eta}{\partial x}-\frac{g \cos \alpha}{\rho_{\circ}} \int_{\eta}^{z} \frac{\partial \rho}{\partial x} d z+f \bar{v}+g \sin \alpha \\
& +\frac{1}{\rho_{\circ}} \frac{\partial\left(\tau_{x x}\right)}{\partial x}+\frac{1}{\rho_{\circ}} \frac{\partial\left(\tau_{x y}\right)}{\partial y}+\frac{1}{\rho_{\circ}} \frac{\partial\left(\tau_{x z}\right)}{\partial z}
\end{aligned}
$$

The Y-Momentum:

$$
\begin{aligned}
\frac{\partial \bar{v}}{\partial t}+\bar{u} \frac{\partial \bar{v}}{\partial x}+\bar{v} \frac{\partial \bar{v}}{\partial y}+\bar{w} \frac{\hat{\mathcal{v}}}{\partial z} & =g \cos \alpha \frac{\partial \eta}{\partial y}-\frac{g \cos \alpha}{\rho_{\circ}} \int_{\eta}^{z} \frac{\partial \rho}{\partial y} d z-f \bar{u} \\
& +\frac{1}{\rho_{\circ}} \frac{\partial\left(\tau_{y x}\right)}{\partial x}+\frac{1}{\rho_{\circ}} \frac{\partial\left(\tau_{y y}\right)}{\partial y}+\frac{1}{\rho_{\circ}} \frac{\partial\left(\tau_{y z}\right)}{\partial z}
\end{aligned}
$$

Converting $\mathrm{X}$ and $\mathrm{Y}$-Momentum equation into finite difference form as follows:

$$
\begin{gathered}
\bar{u}_{i, j, k}^{n+1}=\bar{u}_{i, j, k}^{n}+\Delta t\left[\begin{array}{l}
-\bar{u} \frac{\partial \bar{u}}{\partial x}-\bar{v} \frac{\partial \bar{u}}{\partial y}-\bar{w} \frac{\partial \bar{u}}{\partial z}+f \bar{v}+g \sin \alpha+g \cos \alpha \frac{\partial \eta}{\partial x} \\
\left.-\frac{g \cos \alpha}{\rho_{\circ}} \int_{\eta}^{z} \frac{\partial \rho}{\partial x} d z+\frac{1}{\rho_{\circ}} \frac{\partial\left(\tau_{x x}\right)}{\partial x}+\frac{1}{\rho_{\circ}} \frac{\partial\left(\tau_{x y}\right)}{\partial y}+\frac{1}{\rho_{\circ}} \frac{\partial\left(\tau_{x z}\right)}{\partial z}\right]_{i, j, k}^{n}
\end{array}\right. \\
\bar{v}_{i, j, k}^{n+1}=\bar{v}_{i, j, k}^{n}+\Delta t\left[\begin{array}{l}
-\bar{u} \frac{\partial}{\partial x}-\bar{v} \frac{\partial}{\partial y}-\bar{w} \frac{\partial \bar{v}}{\partial z}-f \bar{u}+g \cos \alpha \frac{\partial \eta}{\partial y}-\frac{g \cos \alpha}{\rho_{\circ}} \int_{\eta}^{z} \frac{\partial \rho}{\partial y} d z \\
+\frac{1}{\rho_{\circ}} \frac{\partial\left(\tau_{y x}\right)}{\partial x}+\frac{1}{\rho_{\circ}} \frac{\partial\left(\tau_{y y}\right)}{\partial y}+\frac{1}{\rho_{\circ}} \frac{\partial\left(\tau_{y z}\right)}{\partial z}
\end{array}\right]_{i, j, k}^{n}
\end{gathered}
$$

Assuming, 


$$
\begin{gathered}
F_{x}=-\bar{u} \frac{\partial \bar{u}}{\partial x}-\bar{v} \frac{\partial \bar{u}}{\partial y}-\bar{w} \frac{\partial \bar{u}}{\partial z}+\frac{1}{\rho_{\circ}} \frac{\partial\left(\tau_{x x}\right)}{\partial x}+\frac{1}{\rho_{\circ}} \frac{\partial\left(\tau_{x y}\right)}{\partial y}+f \bar{v}+g \sin \alpha \\
F_{y}=-\bar{u} \frac{\partial \bar{v}}{\partial x}-\bar{v} \frac{\partial \bar{v}}{\partial y}-\bar{w} \frac{\partial \bar{v}}{\partial z}+\frac{1}{\rho_{\circ}} \frac{\partial\left(\tau_{y x}\right)}{\partial x}+\frac{1}{\rho_{\circ}} \frac{\partial\left(\tau_{y y}\right)}{\partial y}-f \bar{u}
\end{gathered}
$$

The finite difference form of $\mathrm{X}$ and $\mathrm{Y}$-Momentum equation becomes as follows after substituting $F_{x}$ and $F_{y}$ :

$$
\begin{aligned}
& \bar{u}_{i, j, k}^{n+1}=\bar{u}_{i, j, k}^{n}+\Delta t\left[F_{x}+g \cos \alpha \frac{\partial \eta}{\partial x}-\frac{g \cos \alpha}{\rho_{\circ}} \int_{\eta}^{z} \frac{\partial \rho}{\partial x} d z+\frac{1}{\rho_{\circ}} \frac{\partial\left(\tau_{x z}\right)}{\partial z}\right]_{i, j, k}^{n} \\
& \bar{v}_{i, j, k}^{n+1}=\bar{v}_{i, j, k}^{n}+\Delta t\left[F_{y}+g \cos \alpha \frac{\partial \eta}{\partial y}-\frac{g \cos \alpha}{\rho_{\circ}} \int_{\eta}^{z} \frac{\partial \rho}{\partial y} d z+\frac{1}{\rho_{\circ}} \frac{\partial\left(\tau_{y z}\right)}{\partial z}\right]_{i, j, k}^{n}
\end{aligned}
$$

Substituting $\bar{u}_{i, j, j}^{n+1}$ and $\bar{v}_{i, j, j}^{n+1}$ into free water surface equation for $\bar{u}$ and $\bar{v}$, respectively, the free water surface equation becomes,

$$
\begin{aligned}
\frac{\partial \eta}{\partial t} & =\frac{\partial}{\partial x} \int_{\eta}^{h} \bar{u}_{i, j, k}^{n} d z+\left.\Delta t \frac{\partial}{\partial x} \int_{\eta}^{h} F_{x}\right|_{i, j, k} ^{n} d z+\left.\Delta t \frac{\partial}{\partial x} \int_{\eta}^{h} g \cos \alpha \frac{\partial \eta}{\partial x}\right|_{i, j} ^{n} d z-\left.\Delta t \frac{\partial}{\partial x} \int_{\eta}^{h} \frac{g \cos \alpha_{x}}{\rho_{\circ}} \int_{\eta}^{z} \frac{\partial \rho}{\partial x} d z\right|_{i, j, k} ^{n} d z \\
& +\left.\Delta t \frac{\partial}{\partial x} \int_{\eta}^{h} \frac{1}{\rho_{\circ}} \frac{\partial\left(\tau_{x z}\right)}{\partial z}\right|_{i, j, k} ^{n} d z+\frac{\partial}{\partial y} \int_{\eta}^{h} \bar{v}_{i, j, k}^{n} d z+\left.\Delta t \frac{\partial}{\partial y} \int_{\eta}^{h} F_{y}\right|_{i, j, k} ^{n} d z+\left.\Delta t \frac{\partial}{\partial y} \int_{\eta}^{h} g \cos \alpha \frac{\partial \eta}{\partial y}\right|_{i, j} ^{n} d z \\
& -\left.\Delta t \frac{\partial}{\partial y} \int_{\eta}^{h} \frac{g \cos \alpha_{y}}{\rho_{\circ}} \int_{\eta}^{z} \frac{\partial \rho}{\partial y} d z\right|_{i, j, k} ^{n} d z+\left.\Delta t \frac{\partial}{\partial y} \int_{\eta}^{h} \frac{1}{\rho_{\circ}} \frac{\partial\left(\tau_{y z}\right)}{\partial z}\right|_{i, j, k} ^{n} d z
\end{aligned}
$$

By defining new variables $\left.H_{r x}\right|_{i, j, k}$ and $\left.H_{r y}\right|_{i, j, k}$, 


$$
\begin{aligned}
& \left.H_{r x}\right|_{i, j, k}=\frac{\left.H\right|_{i, j, k}+\left.H\right|_{i+1, j, k}}{2} \\
& \left.H_{r y}\right|_{i, j, k}=\frac{\left.H\right|_{i, j, k}+\left.H\right|_{i, j+1, k}}{2}
\end{aligned}
$$

, and by assuming $k t=1$ and $k b(i, j)=K$, the terms are simplified as follows:

$$
\begin{aligned}
\frac{\partial}{\partial x} \int_{\eta}^{h} \bar{u}_{i, j, k}^{n} d z=\left.\frac{\partial}{\partial x} \sum_{k=1}^{K} \bar{u} H_{r x}\right|_{i, j}=\frac{1}{\Delta x}\left[\left.\sum_{k=1}^{K} \bar{u} H_{r x}\right|_{i, j}-\left.\sum_{k=1}^{K} \bar{u} H_{r x}\right|_{i-1, j}\right] \\
\begin{aligned}
\left.\Delta t \frac{\partial}{\partial x} \int_{\eta}^{h} F_{x}\right|_{i, j, k} d z=\Delta t & \left.\frac{\partial}{\partial x} \sum_{k=1}^{K} F_{x} H_{r x}\right|_{i, j}=\frac{\Delta t}{\Delta x}\left[\left.\sum_{k=1}^{K} F_{x} H_{r x}\right|_{i, j}-\left.\sum_{k=1}^{K} F_{x} H_{r x}\right|_{i-1, j}\right] \\
\left.\Delta t \frac{\partial}{\partial x} \int_{\eta}^{h} g \cos \alpha \frac{\partial \eta}{\partial x}\right|_{i, j} ^{n} d z & =\Delta t g \cos \alpha \frac{\partial}{\partial x}\left[\frac{\partial \eta}{\partial x} \int_{\eta}^{h} d z\right]_{i, j}^{n} \\
= & \left.\Delta t g \cos \alpha \frac{\partial \eta}{\partial x}\right|_{i, j} ^{n} \frac{\partial}{\partial x} \int_{\eta}^{h} d z+\left.\Delta t g \cos \alpha \int_{\eta}^{h} d z \frac{\partial^{2} \eta}{\partial x^{2}}\right|_{i, j} ^{n} \\
& =\Delta t g \cos \alpha \frac{\eta_{i, j}^{n}-\eta_{i-1, j}^{n}}{\Delta x} \frac{\left.\int_{\eta}^{h} d z\right|_{i, j} ^{n}-\left.\int_{\eta}^{h} d z\right|_{i-1, j}}{\Delta x} \\
& +\Delta t g \cos \alpha \frac{\eta_{i+1, j}^{n}-2 \eta_{i, j}^{n}+\left.\eta_{i-1, j}^{n} \int_{\eta}^{h} d z\right|_{i, j}}{\Delta x^{2}} \\
& =\frac{\Delta t g \cos \alpha}{\Delta x^{2}}\left[\left.\eta_{i-1, j}^{n} \sum_{k=1}^{K} H\right|_{i-1, j}-\eta_{i, j}^{n}\left(\left.\sum_{k=1}^{K} H\right|_{i, j}+\left.\sum_{k=1}^{K} H\right|_{i-1, j}\right)+\left.\eta_{i+1, j}^{n} \sum_{k=1}^{K} H\right|_{i, j}\right]
\end{aligned}
\end{aligned}
$$




$$
\begin{aligned}
& \left.\Delta t \frac{\partial}{\partial x} \int_{\eta}^{h} \frac{g \cos \alpha_{x}}{\rho_{\circ}} \int_{\eta}^{z} \frac{\partial \rho}{\partial x} d z\right|_{i, j, k} ^{n} d z=\left.\frac{\Delta \operatorname{tg} \cos \alpha}{\rho_{\circ}} \frac{\partial}{\partial x} \int_{\eta}^{h} \int_{\eta}^{z} \frac{\partial \rho}{\partial x} d z\right|_{i, j, k} d z \\
& =\left.\frac{\Delta t g \cos \alpha}{\rho_{\circ}} \frac{\partial}{\partial x} \int_{\eta}^{h} \sum_{k=1}^{K} \frac{\partial \rho}{\partial x} H\right|_{i, j} d z \\
& =\left.\frac{\Delta t g \cos \alpha}{\rho_{\circ}} \frac{\partial}{\partial x} \sum_{k=1}^{K} \sum_{k=1}^{K} \frac{\partial \rho}{\partial x} H H\right|_{i, j} \\
& =\frac{\Delta \operatorname{tg} \cos \alpha}{\rho_{\mathrm{o}} \Delta x} \sum_{k=1}^{K}\left(\left.\sum_{k=1}^{K} \frac{\partial \rho}{\partial x} H^{2}\right|_{i, j}-\left.\sum_{k=1}^{K} \frac{\partial \rho}{\partial x} H^{2}\right|_{i-1, j}\right) \\
& \left.\Delta t \frac{\partial}{\partial x} \int_{\eta}^{h} \frac{1}{\rho_{\circ}} \frac{\partial\left(\tau_{x z}\right)}{\partial z}\right|_{i, j, k} ^{n} d z=\frac{\Delta t}{\rho_{\circ}} \frac{\partial}{\partial x}\left[\left.\tau_{x z}\right|_{h}-\left.\tau_{x z}\right|_{\eta}\right]_{i, j} \\
& =\frac{\Delta t}{\rho_{\mathrm{o}}} \frac{\partial}{\partial x}\left[\tau_{b x}-\tau_{s x}\right]_{i, j} \\
& =\frac{\Delta t}{\rho_{\mathrm{o}} \Delta x}\left[\left(\tau_{b x}-\tau_{s x}\right)_{i, j}-\left(\tau_{b x}-\tau_{s x}\right)_{i-1, j}\right]
\end{aligned}
$$

The same simplifications are applied to all terms that are related to Y-Momentum. Also, a backward difference is used for the unsteady term,

$$
\frac{\partial \eta}{\partial t}=\frac{\eta_{i, j}^{n}-\eta_{i, j}^{n-1}}{\Delta t}
$$

Substituting all the above terms in the free surface finite difference form, multiplying both sides by $\Delta t \Delta x \Delta y$, and re-arranging terms,

$$
\begin{aligned}
& {\left[\left.\frac{-\Delta t^{2} \Delta y g \cos \alpha}{\Delta x} \sum_{k=1}^{K} H\right|_{i-1, j}\right] \eta_{i-1, j}^{n}+\left[\left.\frac{-\Delta t^{2} \Delta x g \cos \alpha}{\Delta y} \sum_{k=1}^{K} H\right|_{i, j-1}\right] \eta_{i, j-1}^{n}+} \\
& {\left[\Delta x \Delta y+\frac{\Delta t^{2} \Delta y g \cos \alpha}{\Delta x}\left(\left.\sum_{k=1}^{K} H\right|_{i, j}+\left.\sum_{k=1}^{K} H\right|_{i-1, j}\right)+\frac{\Delta t^{2} \Delta x g \cos \alpha}{\Delta y}\left(\left.\sum_{k=1}^{K} H\right|_{i, j}+\left.\sum_{k=1}^{K} H\right|_{i, j-1}\right)\right] \eta_{i, j}^{n}+} \\
& {\left[\left.\frac{-\Delta t^{2} \Delta x g \cos \alpha}{\Delta y} \sum_{k=1}^{K} H\right|_{i, j}\right] \eta_{i, j+1}^{n}+\left[\left.\frac{-\Delta t^{2} \Delta y g \cos \alpha}{\Delta x} \sum_{k=1}^{K} H\right|_{i, j}\right] \eta_{i+1, j}^{n}=\Delta t \Delta x \Delta y[R H S]_{i, j, k}^{n}+\Delta x \Delta y \eta_{i, j}^{n-1}}
\end{aligned}
$$


Where:

$$
\begin{aligned}
R H S & =\frac{1}{\Delta x}\left[\left.\sum_{k=1}^{K} \bar{u} H_{r x}\right|_{i, j}-\left.\sum_{k=1}^{K} \bar{u} H_{r x}\right|_{i-1, j}\right]+\frac{1}{\Delta y}\left[\left.\sum_{k=1}^{K} \bar{v} H_{r y}\right|_{i, j}-\left.\sum_{k=1}^{K} \bar{v} H_{r y}\right|_{i, j-1}\right] \\
& +\frac{\Delta t}{\Delta x}\left[\left.\sum_{k=1}^{K} F_{x} H_{r x}\right|_{i, j}-\left.\sum_{k=1}^{K} F_{x} H_{r x}\right|_{i-1, j}\right]+\frac{\Delta t}{\Delta y}\left[\left.\sum_{k=1}^{K} F_{y} H_{r y}\right|_{i, j}-\left.\sum_{k=1}^{K} F_{y} H_{r y}\right|_{i, j-1}\right] \\
& -\frac{\Delta t g \cos \alpha}{\rho_{\mathrm{o}} \Delta x} \sum_{k=1}^{K}\left(\left.\sum_{k=1}^{K} \frac{\partial \rho}{\partial x} H^{2}\right|_{i, j}-\left.\sum_{k=1}^{K} \frac{\partial \rho}{\partial x} H^{2}\right|_{i-1, j}\right) \\
& -\frac{\Delta t g \cos \alpha}{\rho_{\mathrm{o}} \Delta y} \sum_{k=1}^{K}\left(\left.\sum_{k=1}^{K} \frac{\partial \rho}{\partial y} H^{2}\right|_{i, j}-\left.\sum_{k=1}^{K} \frac{\partial \rho}{\partial y} H^{2}\right|_{i, j-1}\right) \\
& +\frac{\Delta t}{\rho_{\mathrm{o}} \Delta x}\left[\left(\tau_{b x}-\tau_{s x}\right)_{i, j}-\left(\tau_{b x}-\tau_{s x}\right)_{i-1, j}\right]+\frac{\Delta t}{\rho_{\mathrm{o}} \Delta y}\left[\left(\tau_{b y}-\tau_{s y}\right)_{i, j}-\left(\tau_{b y}-\tau_{s y}\right)_{i, j-1}\right]
\end{aligned}
$$

For more simplification, the above equation can be written as follows:

$$
W \eta_{i-1, j}^{n}+N \eta_{i, j-1}^{n}+C \eta_{i, j}^{n}+S \eta_{i, j+1}^{n}+E \eta_{i+1, j}^{n}=R
$$

Where:

$$
\begin{aligned}
& W=\left[\left.\frac{-\Delta t^{2} \Delta y g \cos \alpha}{\Delta x} \sum_{k=1}^{K} H\right|_{i-1, j}\right] \\
& N=\left[\left.\frac{-\Delta t^{2} \Delta x g \cos \alpha}{\Delta y} \sum_{k=1}^{K} H\right|_{i, j-1}\right] \\
& C=\left[\Delta x \Delta y+\frac{\Delta t^{2} \Delta y g \cos \alpha}{\Delta x}\left(\left.\sum_{k=1}^{K} H\right|_{i, j}+\left.\sum_{k=1}^{K} H\right|_{i-1, j}\right)+\frac{\Delta t^{2} \Delta x g \cos \alpha}{\Delta y}\left(\left.\sum_{k=1}^{K} H\right|_{i, j}+\left.\sum_{k=1}^{K} H\right|_{i, j-1}\right)\right] \\
& S=\left[\left.\frac{-\Delta t^{2} \Delta x g \cos \alpha}{\Delta y} \sum_{k=1}^{K} H\right|_{i, j}\right] \\
& E=\left[\left.\frac{-\Delta t^{2} \Delta y g \cos \alpha}{\Delta x} \sum_{k=1}^{K} H\right|_{i, j}\right]
\end{aligned}
$$




$$
\begin{aligned}
R & =\Delta t \Delta y\left[\left.\sum_{k=1}^{K} \bar{u} H_{r x}\right|_{i, j}-\left.\sum_{k=1}^{K} \bar{u} H_{r x}\right|_{i-1, j}\right]+\Delta t \Delta x\left[\left.\sum_{k=1}^{K} \bar{v} H_{r y}\right|_{i, j}-\left.\sum_{k=1}^{K} \bar{v} H_{r y}\right|_{i, j-1}\right] \\
& +\Delta t^{2} \Delta y\left[\left.\sum_{k=1}^{K} F_{x} H_{r x}\right|_{i, j}-\left.\sum_{k=1}^{K} F_{x} H_{r x}\right|_{i-1, j}\right]+\Delta t^{2} \Delta x\left[\left.\sum_{k=1}^{K} F_{y} H_{r y}\right|_{i, j}-\left.\sum_{k=1}^{K} F_{y} H_{r y}\right|_{i, j-1}\right] \\
& -\frac{\Delta t^{2} \Delta y g \cos \alpha}{\rho_{\circ}} \sum_{k=1}^{K}\left(\left.\sum_{k=1}^{K} \frac{\partial \rho}{\partial x} H^{2}\right|_{i, j}-\left.\sum_{k=1}^{K} \frac{\partial \rho}{\partial x} H^{2}\right|_{i-1, j}\right) \\
& -\frac{\Delta t^{2} \Delta x g \cos \alpha}{\rho_{\circ}} \sum_{k=1}^{K}\left(\left.\sum_{k=1}^{K} \frac{\partial \rho}{\partial y} H^{2}\right|_{i, j}-\left.\sum_{k=1}^{K} \frac{\partial \rho}{\partial y} H^{2}\right|_{i, j-1}\right) \\
& +\frac{\Delta t^{2} \Delta y}{\rho_{\circ}}\left[\left(\tau_{b x}-\tau_{s x}\right)_{i, j}-\left(\tau_{b x}-\tau_{s x}\right)_{i-1, j}\right]+\frac{\Delta t^{2} \Delta x}{\rho \circ}\left[\left(\tau_{b y}-\tau_{s y}\right)_{i, j}-\left(\tau_{b y}-\tau_{s y}\right)_{i, j-1}\right] \\
& +\Delta x \Delta y \eta_{i, j}^{n-1}
\end{aligned}
$$

$F_{x}$ and $F_{y}$ are explicit operators that account for the advection, horizontal and vertical turbulent shear stresses, Coriolis, and gravitational acceleration component. These operators are converted to a finite difference form and calculated at the cells faces in order to use it in the free surface calculation. Then, by using the upwind difference scheme for the advection terms, each term is discretized as follows:

For $\bar{u}_{i, j, k}>0$,

$$
\left.\bar{u} \frac{\partial \bar{u}}{\partial x}\right|_{i, j, k} ^{n}=\left[\bar{u}_{i, j, k}\left(\frac{\bar{u}_{i, j, k}-\bar{u}_{i-1, j, k}}{\Delta x}\right)\right]^{n}
$$

For $\bar{u}_{i, j, k}<0$,

$$
\left.\bar{u} \frac{\partial u}{\partial x}\right|_{i, j, k} ^{n}=\left[\bar{u}_{i, j, k}\left(\frac{\bar{u}_{i+1, j, k}-\bar{u}_{i, j, k}}{\Delta x}\right)\right]^{n}
$$


We need to define $[[a 1, a 2]]$ and $((a 1, a 2))$ to be the maximum and minimum value of $(a 1$ and $a 2$ ), respectively. Then, a general expression for the above two cases can be represented in one expression as follows:

$\left.\bar{u} \frac{\partial \bar{u}}{\partial x}\right|_{i, j, k} ^{n}=\left[\frac{\bar{u}_{i, j, k}\left[\left[\bar{u}_{i, j, k}, 0\right]\right]-\bar{u}_{i, j, k}\left(\left(\bar{u}_{i, j, k}, 0\right)\right)-\bar{u}_{i-1, j, k}\left[\left[\bar{u}_{i, j, k}, 0\right]\right]+\bar{u}_{i+1, j, k}\left(\left(\bar{u}_{i, j, k}, 0\right)\right)}{\Delta x}\right]^{n}$

Where: the term $\left[\left[\bar{u}_{i, j, k}, 0\right]\right]$ and $\left(\left(\bar{u}_{i, j, k}, 0\right)\right)$ represent a single positive and negative value of $\bar{u}_{i, j, k}$, respectively.

In the same way, the other advection terms are

$$
\begin{aligned}
& \left.\bar{v} \frac{\partial \bar{u}}{\partial y}\right|_{i, j, k} ^{n}=\left[\frac{\bar{u}_{i, j, k}\left[\left[\bar{v}_{i, j, k}, 0\right]\right]-\bar{u}_{i, j, k}\left(\left(\bar{v}_{i, j, k}, 0\right)\right)-\bar{u}_{i, j-1, k}\left[\left[\bar{v}_{i, j, k}, 0\right]\right]+\bar{u}_{i, j+1, k}\left(\left(\bar{v}_{i, j, k}, 0\right)\right)}{\Delta y}\right]^{n} \\
& \left.\bar{w} \frac{\partial \bar{u}}{\partial z}\right|_{i, j, k} ^{n}=\left[\frac{\bar{u}_{i, j, k}\left[\left[\bar{w}_{i, j, k}, 0\right]\right]-\bar{u}_{i, j, k}\left(\left(\bar{w}_{i, j, k}, 0\right)\right)-\bar{u}_{i, j, k-1}\left[\left[\bar{w}_{i, j, k}, 0\right]\right]+\bar{u}_{i, j, k+1}\left(\left(\bar{w}_{i, j, k}, 0\right)\right)}{\Delta z}\right]^{n}
\end{aligned}
$$

By using the central difference scheme for diffusion terms, each term is discretized as follows:

$$
\begin{aligned}
& \left.\frac{1}{\rho_{\circ}} \frac{\partial\left(\tau_{x x}\right)}{\partial x}\right|_{i, j, k} ^{n}=\frac{v_{h}}{\rho_{i, j, k} \Delta x_{i, j, k}}\left[\frac{\bar{u}_{i+1, j, k}-\bar{u}_{i, j, k}}{\Delta x_{i+1 / 2, j, k}}-\frac{\bar{u}_{i, j, k}-\bar{u}_{i-1, j, k}}{\Delta x_{i-1 / 2, j, k}}\right]^{n} \\
& \left.\frac{1}{\rho_{\circ}} \frac{\partial\left(\tau_{x y}\right)}{\partial y}\right|_{i, j, k} ^{n}=\frac{v_{h}}{\rho_{i, j, k} \Delta y_{i, j, k}}\left[\frac{\bar{u}_{i, j+1, k}-\bar{u}_{i, j, k}}{\Delta y_{i, j+1 / 2, k}}-\frac{\bar{u}_{i, j, k}-\bar{u}_{i, j-1, k}}{\Delta y_{i, j-1 / 2, k}}\right]^{n}
\end{aligned}
$$

By collecting terms and adding Coriolis and gravitational acceleration component, $F_{x}$ will be as follows: 


$$
\begin{aligned}
\left.F_{x}\right|_{i, j, k} ^{n}= & -\left[\frac{\bar{u}_{i, j, k}[}{\left.\left[\bar{u}_{i, j, k}, 0\right]\right]-\bar{u}_{i, j, k}\left(\left(\bar{u}_{i, j, k}, 0\right)\right)-\bar{u}_{i-1, j, k}\left[\left[\bar{u}_{i, j, k}, 0\right]\right]+\bar{u}_{i+1, j, k}\left(\left(\bar{u}_{i, j, k}, 0\right)\right)} \Delta\right]^{n} \\
& -\left[\frac{\bar{u}_{i, j, k}\left[\left[\bar{v}_{i, j, k}, 0\right]\right]-\bar{u}_{i, j, k}\left(\left(\bar{v}_{i, j, k}, 0\right)\right)-\bar{u}_{i, j-1, k}\left[\left[\bar{v}_{i, j, k}, 0\right]\right]+\bar{u}_{i, j+1, k}\left(\left(\bar{v}_{i, j, k}, 0\right)\right)}{\Delta y}\right]^{n} \\
& -\left[\frac{\bar{u}_{i, j, k}\left[\left[\bar{w}_{i, j, k}, 0\right]\right]-\bar{u}_{i, j, k}\left(\left(\bar{w}_{i, j, k}, 0\right)\right)-\bar{u}_{i, j, k-1}\left[\left[\bar{w}_{i, j, k}, 0\right]\right]+\bar{u}_{i, j, k+1}\left(\left(\bar{w}_{i, j, k}, 0\right)\right)}{\Delta z}\right]^{n} \\
& +\frac{v_{h}}{\rho_{i, j, k} \Delta x_{i, j, k}}\left[\frac{\bar{u}_{i+1, j, k}-\bar{u}_{i, j, k}}{\Delta x_{i+1 / 2, j, k}}-\frac{\bar{u}_{i, j, k}-\bar{u}_{i-1, j, k}}{\Delta x_{i-1 / 2, j, k}}\right]^{n} \\
& +\frac{v_{h}}{\rho_{i, j, k} \Delta y_{i, j, k}}\left[\frac{\bar{u}_{i, j+1, k}-\bar{u}_{i, j, k}}{\Delta y_{i, j+1 / 2, k}}-\frac{\bar{u}_{i, j, k}-\bar{u}_{i, j-1, k}}{\Delta y_{i, j-1 / 2, k}}\right]^{n} \\
& +f \bar{v}_{i, j, k}^{n}+g \sin \alpha
\end{aligned}
$$

In the same way, $F_{y}$ will be as follows:

$$
\begin{aligned}
\left.F_{y}\right|_{i, j, k} ^{n}= & -\left[\frac{\bar{v}_{i, j, k}\left[\left[\bar{u}_{i, j, k}, 0\right]\right]-\bar{v}_{i, j, k}\left(\left(\bar{u}_{i, j, k}, 0\right)\right)-\bar{v}_{i, j-1, k}\left[\left[\bar{u}_{i, j, k}, 0\right]\right]+\bar{v}_{i, j+1, k}\left(\left(\bar{u}_{i, j, k}, 0\right)\right)}{\Delta x}\right]^{n} \\
& -\left[\frac{\bar{v}_{i, j, k}\left[\left[\bar{v}_{i, j, k}, 0\right]\right]-\bar{v}_{i, j, k}\left(\left(\bar{v}_{i, j, k}, 0\right)\right)-\bar{v}_{i, j-1, k}\left[\left[\bar{v}_{i, j, k}, 0\right]\right]+\bar{v}_{i, j+1, k}\left(\left(\bar{v}_{i, j, k}, 0\right)\right)}{\Delta y}\right]^{n} \\
& -\left[\frac{\bar{v}_{i, j, k}\left[\left[\bar{w}_{i, j, k}, 0\right]\right]-\bar{v}_{i, j, k}\left(\left(\bar{w}_{i, j, k}, 0\right)\right)-\bar{v}_{i, j, k-1}\left[\left[\bar{w}_{i, j, k}, 0\right]\right]+\bar{v}_{i, j, k+1}\left(\left(\bar{w}_{i, j, k}, 0\right)\right)}{\Delta z}\right]^{n} \\
& +\frac{v_{h}}{\rho_{i, j, k} \Delta x_{i, j, k}}\left[\frac{\bar{v}_{i+1, j, k}-\bar{v}_{i, j, k}}{\Delta x_{i+1 / 2, j, k}}-\frac{\bar{v}_{i, j, k}-\bar{v}_{i-1, j, k}}{\Delta x_{i-1 / 2, j, k}}\right]^{n} \\
& +\frac{v_{h}}{\rho_{i, j, k} \Delta y_{i, j, k}}\left[\frac{\bar{v}_{i, j+1, k}-\bar{v}_{i, j, k}}{\Delta y_{i, j+1 / 2, k}}-\frac{\bar{v}_{i, j, k}-\bar{v}_{i, j-1, k}}{\Delta y_{i, j-1 / 2, k}}\right]^{n} \\
& -f \bar{u}_{i, j, k}^{n}
\end{aligned}
$$




\subsubsection{Numerical Solution of the Momentum Equations}

After solving the free surface equation and determining $\eta_{i, j}^{n}$ at the center of each cell, the $\mathrm{X}$ and Y-Momentum equation are solved numerically to get $\bar{u}_{i, j, k}^{n+1}$ and $\bar{v}_{i, j, k}^{n+1}$ at the cells faces depending on known values of $\eta_{i, j}^{n}, \bar{u}_{i, j, k}^{n} \bar{v}_{i, j, k}^{n}$ from the previous time level. The solution of the $\mathrm{X}$ and $\mathrm{Y}$-Momentum equation was done by employing the time splitting technique.

The X-Momentum equation is

$$
\begin{aligned}
\frac{\partial \bar{u}}{\partial t}+\bar{u} \frac{\partial \bar{u}}{\partial x}+\bar{v} \frac{\partial \bar{u}}{\partial y}+\bar{w} \frac{\partial \bar{u}}{\partial z} & =g \cos \alpha \frac{\partial \eta}{\partial x}-\frac{g \cos \alpha}{\rho_{\circ}} \int_{\eta}^{z} \frac{\partial \rho}{\partial x} d z+f \bar{v}+g \sin \alpha \\
& +\frac{1}{\rho_{\circ}} \frac{\partial\left(\tau_{x x}\right)}{\partial x}+\frac{1}{\rho_{\circ}} \frac{\partial\left(\tau_{x y}\right)}{\partial y}+\frac{1}{\rho_{\circ}} \frac{\partial\left(\tau_{x z}\right)}{\partial z}
\end{aligned}
$$

The equation is split into two equations in two stages at each model time step. One of them is treated explicitly, while the second equation is treated implicitly.

Stage 1:

$$
\begin{aligned}
\frac{\partial \bar{u}}{\partial t} & =-\bar{u} \frac{\partial \bar{u}}{\partial x}-\bar{v} \frac{\partial \bar{u}}{\partial y}-\bar{w} \frac{\partial \bar{u}}{\partial z}+g \cos \alpha \frac{\partial \eta}{\partial x}-\frac{g \cos \alpha}{\rho_{\circ}} \int_{\eta}^{z} \frac{\partial \rho}{\partial x} d z+f \bar{v}+g \sin \alpha \\
& +\frac{1}{\rho_{\circ}} \frac{\partial\left(\tau_{x x}\right)}{\partial x}+\frac{1}{\rho_{\circ}} \frac{\partial\left(\tau_{x y}\right)}{\partial y}+\frac{1}{\rho_{\circ}} \frac{\partial\left(\tau_{s x}+\tau_{b x}\right)}{\partial z}
\end{aligned}
$$

Stage 2:

$$
\frac{\partial \bar{u}}{\partial t}=\frac{1}{\rho_{\circ}} \frac{\partial\left(\tau_{x z}\right)}{\partial z}
$$

In the first stage, the equation is solved explicitly as follows: 


$$
\bar{u}_{i, j, k}^{*}=\bar{u}_{i, j, k}^{n}+\Delta t\left[\begin{array}{l}
-\bar{u} \frac{\partial \bar{u}}{\partial x}-\bar{v} \frac{\partial \bar{u}}{\partial y}-\bar{w} \frac{\partial \bar{u}}{\partial z}+g \cos \alpha \frac{\partial \eta}{\partial x}-\frac{g \cos \alpha}{\rho_{\circ}} \int_{\eta}^{z} \frac{\partial \rho}{\partial x} d z+f \bar{v} \\
+g \sin \alpha+\frac{1}{\rho_{\circ}} \frac{\partial\left(\tau_{x x}\right)}{\partial x}+\frac{1}{\rho_{\circ}} \frac{\partial\left(\tau_{x y}\right)}{\partial y}+\frac{1}{\rho_{\circ}} \frac{\partial\left(\tau_{s x}+\tau_{b x}\right)}{\partial z}
\end{array}\right]_{i, j, k}^{n}
$$

Where: $\bar{u}_{i, j, k}^{*}$ represents the value of $\bar{u}_{i, j, k}$ at $t=t+\Delta t$.

The terms inside the parentheses are simplified as follows:

$$
\begin{aligned}
& \left.g \cos \alpha \frac{\partial \eta}{\partial x}\right|_{i, j, k} ^{n}-\left.\frac{g \cos \alpha}{\rho_{\circ}} \int_{\eta}^{z} \frac{\partial \rho}{\partial x} d z\right|_{i, j, k} ^{n}=\frac{g \cos \alpha}{\Delta x}\left(\eta_{i+1, j, k}^{n}-\eta_{i, j, k}^{n}\right)-\left.\frac{g \cos \alpha}{\rho \Delta x} \sum_{k=1}^{K}\left(\rho_{i+1, j, k}^{n}-\rho_{i, j, k}^{n}\right) H_{r x}\right|_{i, j, k} ^{n} \\
& \left.\frac{1}{\rho_{\circ}} \frac{\partial\left(\tau_{s x}+\tau_{b x}\right)}{\partial z}\right|_{i, j, k} ^{n}=\frac{1}{\rho_{\circ} \Delta z}\left[\left(\tau_{s x}+\tau_{b x}\right)_{i, j, k+1 / 2}-\left(\tau_{s x}+\tau_{b x}\right)_{i, j, k-1 / 2}\right]^{n}
\end{aligned}
$$

The remaining terms are similar to what we did in the solution of the free surface equation.

By collecting all terms, the final explicit finite difference equation of $\bar{u}_{i, j, k}^{*}$ will be as follows: 


$$
\begin{aligned}
\bar{u}_{i, j, k}^{*}= & \bar{u}_{i, j, k}^{n}-\frac{\Delta t}{\Delta x}\left[\bar{u}_{i, j, k}\left[\left[\bar{u}_{i, j, k}, 0\right]\right]-\bar{u}_{i, j, k}\left(\left(\bar{u}_{i, j, k}, 0\right)\right)-\bar{u}_{i-1, j, k}\left[\left[\bar{u}_{i, j, k}, 0\right]\right]+\bar{u}_{i+1, j, k}\left(\left(\bar{u}_{i, j, k}, 0\right)\right)\right]^{n} \\
& -\frac{\Delta t}{\Delta y}\left[\bar{u}_{i, j, k}\left[\left[\bar{v}_{i, j, k}, 0\right]\right]-\bar{u}_{i, j, k}\left(\left(\bar{v}_{i, j, k}, 0\right)\right)-\bar{u}_{i, j-1, k}\left[\left[\bar{v}_{i, j, k}, 0\right]\right]+\bar{u}_{i, j+1, k}\left(\left(\bar{v}_{i, j, k}, 0\right)\right)\right]^{n} \\
& -\frac{\Delta t}{\Delta z}\left[\bar{u}_{i, j, k}\left[\left[\bar{w}_{i, j, k}, 0\right]\right]-\bar{u}_{i, j, k}\left(\left(\bar{w}_{i, j, k}, 0\right)\right)-\bar{u}_{i, j-1, k}\left[\left[\bar{w}_{i, j, k}, 0\right]\right]+\bar{u}_{i, j+1, k}\left(\left(\bar{w}_{i, j, k}, 0\right)\right)\right]^{n} \\
& +\frac{\Delta t g \cos \alpha}{\Delta x}\left(\eta_{i+1, j, k}^{n}-\eta_{i, j, k}^{n}\right)-\left.\frac{\Delta t g \cos \alpha}{\rho \Delta x} \sum_{k=1}^{K}\left(\rho_{i+1, j, k}^{n}-\rho_{i, j, k}^{n}\right) H_{r x}\right|_{i, j, k} ^{n}+\Delta t f \bar{v}_{i, j, k}^{n}+\Delta t g \sin \alpha \\
& +\frac{\Delta t v_{h}}{\Delta x_{i, j, k}}\left[\frac{\bar{u}_{i+1, j, k}-\bar{u}_{i, j, k}}{\Delta x_{i+1 / 2, j, k}}-\frac{\bar{u}_{i, j, k}-\bar{u}_{i-1, j, k}}{\Delta x_{i-1 / 2, j, k}}\right]^{n}+\frac{\Delta t v_{h}}{\Delta y_{i, j, k}}\left[\frac{\bar{u}_{i, j+1, k}-\bar{u}_{i, j, k}}{\Delta y_{i, j+1 / 2, k}}-\frac{\bar{u}_{i, j, k}-\bar{u}_{i, j-1, k}}{\Delta y_{i, j-1 / 2, k}}\right]^{n} \\
& +\frac{\Delta t}{\rho_{\mathrm{o}} \Delta z}\left[\left(\tau_{s x}+\tau_{b x}\right)_{i, j, k+1 / 2}-\left(\tau_{s x}+\tau_{b x}\right)_{i, j, k-1 / 2}\right]^{n}
\end{aligned}
$$

Now, $\bar{u}_{i, j, k}^{*}$ is calculated at each cell face and will be used to calculate $\bar{u}_{i, j, k}^{n+1}$ by solving the equation of the second stage implicitly. In this stage, the equation is solved by using a fully implicit finite difference technique for the vertical diffusion term as follows:

$$
\begin{gathered}
\frac{\partial \bar{u}}{\partial t}=\frac{\partial}{\partial z}\left[v_{v} \frac{\partial \bar{u}}{\partial z}\right] \\
\frac{\bar{u}_{i, j, k}^{n+1}-\bar{u}_{i, j, k}^{*}}{\Delta t}=\frac{1}{\Delta z_{i, j, k}}\left[v_{v_{i, j, k}} \frac{\bar{u}_{i, j, k+1}^{n+1}-\bar{u}_{i, j, k}^{n+1}}{\Delta z_{i, j, k+1 / 2, k}}-v_{v_{i, j, k-1}} \frac{\bar{u}_{i, j, k}^{n+1}-\bar{u}_{i, j, k-1}^{n+1}}{\Delta z_{i, j, k-1 / 2, k}}\right] \\
{\left[-\frac{\Delta t v_{v_{i, j, k-1}}}{\Delta z_{i, j, k} \Delta z_{i, j, k-1 / 2}}\right] \bar{u}_{i, j, k-1}^{n+1}+\left[1+\frac{\Delta t v_{v_{i, j, k-1}}}{\Delta z_{i, j, k} \Delta z_{i, j, k+1 / 2}}+\frac{\Delta t v_{v_{i, j, k}}}{\Delta z_{i, j, k} \Delta z_{i, j, k-1 / 2}}\right] \bar{u}_{i, j, k}^{n+1}} \\
+\left[-\frac{\Delta t v_{v_{i, j, k}}}{\Delta z_{i, j, k} \Delta z_{i, j, k+1 / 2}}\right] \bar{u}_{i, j, k+1}^{n+1}=\bar{u}_{i, j, k}^{*}
\end{gathered}
$$

For more simplification, the above equation can be written as follows:

$$
U \bar{u}_{i, j, k-1}^{n+1}+C \bar{u}_{i, j, k}^{n+1}+D \bar{u}_{i, j, k+1}^{n+1}=R
$$


Where:

$$
\begin{aligned}
& U=\left[-\frac{\Delta t v_{v_{i, j, k-1}}}{\Delta z_{i, j, k} \Delta z_{i, j, k-1 / 2}}\right] \\
& C=\left[1+\frac{\Delta t v_{v_{i, j, k-1}}}{\Delta z_{i, j, k} \Delta z_{i, j, k+1 / 2}}+\frac{\Delta t v_{v_{i, j, k}}}{\Delta z_{i, j, k} \Delta z_{i, j, k-1 / 2}}\right] \\
& D=\left[-\frac{\Delta t v_{v_{i, j, k}}}{\Delta z_{i, j, k} \Delta z_{i, j, k+1 / 2}}\right] \\
& R=\bar{u}_{i, j, k}^{*}
\end{aligned}
$$

A system of linear algebraic equations for each water column in the domain is solved by using Thomas algorithm to calculate $\bar{u}_{i, j, k}^{n+1}$ at the center of each cell face perpendicular to $x$-direction.

Similarly, the Y-Momentum equation is

$$
\begin{aligned}
\frac{\partial \bar{v}}{\partial t}+\bar{u} \frac{\partial \bar{v}}{\partial x}+\bar{v} \frac{\partial \bar{v}}{\partial y}+\bar{w} \frac{\partial \bar{v}}{\partial z} & =g \cos \alpha \frac{\partial \eta}{\partial y}-\frac{g \cos \alpha}{\rho_{\circ}} \int_{\eta}^{z} \frac{\partial \rho}{\partial y} d z-f \bar{u} \\
& +\frac{1}{\rho_{\circ}} \frac{\partial\left(\tau_{y x}\right)}{\partial x}+\frac{1}{\rho_{\circ}} \frac{\partial\left(\tau_{y y}\right)}{\partial y}+\frac{1}{\rho_{\circ}} \frac{\partial\left(\tau_{y z}\right)}{\partial z}
\end{aligned}
$$

This equation is also solved by splitting the equation into two equations in two stages at the same model time step. One of them is treated explicitly, while the second equation is treated implicitly. 
Stage 1:

$$
\begin{aligned}
\frac{\partial \bar{v}}{\partial t} & =-\bar{u} \frac{\partial \bar{v}}{\partial x}-\bar{v} \frac{\partial \bar{v}}{\partial y}-\bar{w} \frac{\partial \bar{v}}{\partial z}+g \cos \alpha \frac{\partial \eta}{\partial y}-\frac{g \cos \alpha}{\rho_{\circ}} \int_{\eta}^{z} \frac{\partial \rho}{\partial y} d z-f \bar{u} \\
& +\frac{1}{\rho_{\circ}} \frac{\partial\left(\tau_{y x}\right)}{\partial x}+\frac{1}{\rho_{\circ}} \frac{\partial\left(\tau_{y y}\right)}{\partial y}+\frac{1}{\rho_{\circ}} \frac{\partial\left(\tau_{s y}+\tau_{b y}\right)}{\partial z}
\end{aligned}
$$

Stage 2:

$$
\frac{\partial \bar{v}}{\partial t}=\frac{1}{\rho_{\circ}} \frac{\partial\left(\tau_{y z}\right)}{\partial z}
$$

In the first stage, the equation is solved explicitly as follows:

$$
\bar{v}_{i, j, k}^{*}=\bar{v}_{i, j, k}^{n}+\Delta t\left[\begin{array}{l}
-\bar{u} \frac{\partial \bar{v}}{\partial x}-\bar{v} \frac{\partial}{\partial y}-\bar{w} \frac{\partial}{\partial z}+g \cos \alpha \frac{\partial \eta}{\partial y}-\frac{g \cos \alpha}{\rho_{\circ}} \int_{\eta}^{z} \frac{\partial \rho}{\partial y} d z-f \bar{u} \\
+\frac{1}{\rho_{\circ}} \frac{\partial\left(\tau_{y x}\right)}{\partial x}+\frac{1}{\rho_{\circ}} \frac{\partial\left(\tau_{y y}\right)}{\partial y}+\frac{1}{\rho_{\circ}} \frac{\partial\left(\tau_{s y}+\tau_{b y}\right)}{\partial z}
\end{array}\right]_{i, j, k}^{n}
$$

Where: $\bar{v}_{i, j, k}^{*}$ represents the value of $\bar{v}_{i, j, k}$ at $t=t+\Delta t$.

The terms inside the parentheses are simplified as follows:

$$
\begin{aligned}
& \left.g \cos \alpha \frac{\partial \eta}{\partial y}\right|_{i, j, k} ^{n}-\left.\frac{g \cos \alpha}{\rho_{\circ}} \int_{\eta}^{z} \frac{\partial \rho}{\partial y} d z\right|_{i, j, k} ^{n}=\frac{g \cos \alpha}{\Delta y}\left(\eta_{i, j+1, k}^{n}-\eta_{i, j, k}^{n}\right)-\left.\frac{g \cos \alpha}{\rho \Delta y} \sum_{k=1}^{K}\left(\rho_{i, j+1, k}^{n}-\rho_{i, j, k}^{n}\right) H_{r y}\right|_{i, j, k} ^{n} \\
& \left.\frac{1}{\rho_{\circ}} \frac{\partial\left(\tau_{s y}+\tau_{b y}\right)}{\partial z}\right|_{i, j, k} ^{n}=\frac{1}{\rho_{\circ} \Delta z}\left[\left(\tau_{s y}+\tau_{b y}\right)_{i, j, k+1 / 2}-\left(\tau_{s y}+\tau_{b y}\right)_{i, j, k-1 / 2}\right]^{n}
\end{aligned}
$$

The remaining terms are similar to what we did in the solution of the free surface equation. 
By collecting all terms, the final explicit finite difference equation of $\bar{v}_{i, j, k}^{*}$ will be as follows:

$$
\begin{aligned}
\bar{v}_{i, j, k}^{*}= & \bar{v}_{i, j, k}^{n}-\frac{\Delta t}{\Delta x}\left[\bar{v}_{i, j, k}\left[\left[\bar{u}_{i, j, k}, 0\right]\right]-\bar{v}_{i, j, k}\left(\left(\bar{u}_{i, j, k}, 0\right)\right)-\bar{v}_{i-1, j, k}\left[\left[\bar{u}_{i, j, k}, 0\right]\right]+\bar{v}_{i+1, j, k}\left(\left(\bar{u}_{i, j, k}, 0\right)\right)\right]^{n} \\
& -\frac{\Delta t}{\Delta y}\left[\bar{v}_{i, j, k}\left[\left[\bar{v}_{i, j, k}, 0\right]\right]-\bar{v}_{i, j, k}\left(\left(\bar{v}_{i, j, k}, 0\right)\right)-\bar{v}_{i, j-1, k}\left[\left[\bar{v}_{i, j, k}, 0\right]\right]+\bar{v}_{i, j+1, k}\left(\left(\bar{v}_{i, j, k}, 0\right)\right)\right]^{n} \\
& -\frac{\Delta t}{\Delta z}\left[\bar{v}_{i, j, k}\left[\left[\bar{w}_{i, j, k}, 0\right]\right]-\bar{v}_{i, j, k}\left(\left(\bar{w}_{i, j, k}, 0\right)\right)-\bar{v}_{i, j-1, k}\left[\left[\bar{w}_{i, j, k}, 0\right]\right]+\bar{v}_{i, j+1, k}\left(\left(\bar{w}_{i, j, k}, 0\right)\right)\right]^{n} \\
& +\frac{\Delta t g \cos \alpha}{\Delta y}\left(\eta_{i, j+1, k}^{n}-\eta_{i, j, k}^{n}\right)-\left.\frac{\Delta t g \cos \alpha}{\rho \Delta y} \sum_{k=1}^{K}\left(\rho_{i, j+1, k}^{n}-\rho_{i, j, k}^{n}\right) H_{r y}\right|_{i, j, k} ^{n}-\Delta t f \bar{u}_{i, j, k}^{n} \\
& +\frac{\Delta t v_{h}}{\Delta x_{i, j, k}}\left[\frac{\bar{v}_{i+1, j, k}-\bar{v}_{i, j, k}}{\Delta x_{i+1 / 2, j, k}}-\frac{\bar{v}_{i, j, k}-\bar{v}_{i-1, j, k}}{\Delta x_{i-1 / 2, j, k}}\right]^{n}+\frac{\Delta t v_{h}}{\Delta y_{i, j, k}}\left[\frac{\bar{v}_{i, j+1, k}-\bar{v}_{i, j, k}}{\Delta y_{i, j+1 / 2, k}}-\frac{\bar{v}_{i, j, k}-\bar{v}_{i, j-1, k}}{\Delta y_{i, j-1 / 2, k}}\right]^{n} \\
& +\frac{\Delta t}{\rho_{\mathrm{o}} \Delta z}\left[\left(\tau_{s y}+\tau_{b y}\right)_{i, j, k+1 / 2}-\left(\tau_{s y}+\tau_{b y}\right)_{i, j, k-1 / 2}\right]^{n}
\end{aligned}
$$

Now, $\bar{v}_{i, j, k}^{*}$ is calculated at each cell face and will be used to calculate $\bar{v}_{i, j, k}^{n+1}$ by solving the equation of the second stage implicitly and as follows:

$$
\begin{gathered}
\frac{\partial \bar{v}}{\partial t}=\frac{\partial}{\partial z}\left[v_{v} \frac{\partial \bar{v}}{\partial z}\right] \\
\frac{\bar{v}_{i, j, k}^{n+1}-\bar{v}_{i, j, k}^{*}}{\Delta t}=\frac{1}{\Delta z_{i, j, k}}\left[v_{v_{i, j, k}} \frac{\bar{v}_{i, j, k+1}^{n+1}-\bar{v}_{i, j, k}^{n+1}}{\Delta z_{i, j, k+1 / 2, k}}-v_{v_{i, j, k-1}} \frac{\bar{v}_{i, j, k}^{n+1}-\bar{v}_{i, j, k-1}^{n+1}}{\Delta z_{i, j, k-1 / 2, k}}\right] \\
{\left[-\frac{\Delta t v_{v_{i, j k-1}}}{\Delta z_{i, j, k} \Delta z_{i, j, k-1 / 2}}\right] \bar{v}_{i, j, k-1}^{n+1}+\left[1+\frac{\Delta t v_{v_{i, j, k-1}}}{\Delta z_{i, j, k} \Delta z_{i, j, k+1 / 2}}+\frac{\Delta t v_{v_{i, j, k}}}{\Delta z_{i, j, k} \Delta z_{i, j, k-1 / 2}}\right] \bar{v}_{i, j, k}^{n+1,}} \\
+\left[-\frac{\Delta t v_{v_{i, j, k}}}{\Delta z_{i, j, k} \Delta z_{i, j, k+1 / 2}}\right] \bar{v}_{i, j, k+1}^{n+1}=\bar{v}_{i, j, k}^{*}
\end{gathered}
$$

For more simplification, the above equation can be written as follows: 


$$
U \bar{v}_{i, j, k-1}^{n+1}+C \bar{v}_{i, j, k}^{n+1}+D \bar{v}_{i, j, k+1}^{n+1}=R
$$

Where:

$$
\begin{aligned}
& U=\left[-\frac{\Delta t v_{v_{i, j, k-1}}}{\Delta z_{i, j, k} \Delta z_{i, j, k-1 / 2}}\right] \\
& C=\left[1+\frac{\Delta t v_{v_{i, j, k-1}}}{\Delta z_{i, j, k} \Delta z_{i, j, k+1 / 2}}+\frac{\Delta t v_{v_{i, j, k}}}{\Delta z_{i, j, k} \Delta z_{i, j, k-1 / 2}}\right] \\
& D=\left[-\frac{\Delta t v_{v_{i, j, k}}}{\Delta z_{i, j, k} \Delta z_{i, j, k+1 / 2}}\right] \\
& R=\bar{v}_{i, j, k}^{*}
\end{aligned}
$$

A system of linear algebraic equations for each water column in the domain is solved by using Thomas algorithm to calculate $\bar{v}_{i, j, k}^{n+1}$ at the center of each cell face perpendicular to $y$-direction. 


\subsubsection{Model Implementation of the Semi-Implicit Finite Differences Scheme}

The numerical solution of the free surface equation discretized the surface elevation implicitly, while the numerical solution of the momentum equations treated the water surface elevation term explicitly. Fully implicit discretization of the free surface equation results in free surface wave damping regardless the approach that could be adopted to treat the free surface elevation gradient of the momentum equations, explicitly (Scott A. Wells, 2002b) or implicitly (V. Casulli \& Cattani, 1994; Vincenzo Casulli \& Cheng, 1992). Thus, an issue associated with solving the free surface equation fully implicitly is the diffusive of the surface wave predictions (Scott A. Wells, 2002b). The higher the time step is, the more the free surface wave damping is. In an attempt to reduce the amount of damping, a new scheme will be derived based on the inclusion of a semi-implicit parameter for the free surface elevation gradient terms.

The semi-implicit scheme bases is first proposed by Vincenzo Casulli and Cheng (1992) and since then it has been implemented by many modelers, see Section 1.1. A more accurate general scheme for solving the three-dimensional hydrodynamic equations is done by discretizing the free surface elevation gradient using the degree of implicitness $(\theta$-method) as in the semi-implicit scheme of Casulli and Cattani (1994), in which the scheme is stable for $1 / 2 \leq \theta \leq 1$ and unstable for $\theta<1 / 2$.

The previous 3D formulation was based on the 2D CE-QUAL-W2 fully implicit scheme. The fully implicit scheme solves for the free surface elevation implicitly from the free surface equation, but the solution of the momentum equations treats the free surface elevation explicitly. In order to make both solutions linked in which the free surface 
elevation is treated either explicitly or implicitly at the same time step, a semi-implicit scheme for the free surface elevation gradient was employed in both solutions of free surface elevation and momentums.

The inclusion of the degree of implicitness $(\theta)$ to the previous formulations is as follows:

$$
\begin{aligned}
& \bar{u}_{i, j, k}^{n+1}=\bar{u}_{i, j, k}^{n}+\Delta t\left[\begin{array}{l}
-\bar{u} \frac{\partial \bar{u}}{\partial x}-\bar{v} \frac{\partial \bar{u}}{\partial y}-\bar{w} \frac{\partial \bar{u}}{\partial z}+f \bar{v}+g \sin \alpha+(1-\theta) g \cos \alpha \frac{\partial \eta}{\partial x} \\
-\frac{g \cos \alpha}{\rho_{\circ}} \int_{\eta}^{z} \frac{\partial \rho}{\partial x} d z+\frac{1}{\rho_{\circ}} \frac{\partial\left(\tau_{x x}\right)}{\partial x}+\frac{1}{\rho_{\circ}} \frac{\partial\left(\tau_{x y}\right)}{\partial y}+\frac{1}{\rho_{\circ}} \frac{\partial\left(\tau_{x z}\right)}{\partial z}
\end{array}\right]_{i, j, k}^{n} \\
& +\Delta t\left[\theta g \cos \alpha \frac{\partial \eta}{\partial x}\right]_{i, j, k}^{n+1} \\
& \bar{v}_{i, j, k}^{n+1}=\bar{v}_{i, j, k}^{n}+\Delta t\left[\begin{array}{l}
-\bar{u} \frac{\partial \bar{v}}{\partial x}-\bar{v} \frac{\partial \bar{v}}{\partial y}-\bar{w} \frac{\partial}{\partial z}-f \bar{u}+(1-\theta) g \cos \alpha \frac{\partial \eta}{\partial y} \\
-\frac{g \cos \alpha}{\rho_{\circ}} \int_{\eta}^{z} \frac{\partial \rho}{\partial y} d z+\frac{1}{\rho_{\circ}} \frac{\partial\left(\tau_{y x}\right)}{\partial x}+\frac{1}{\rho_{\circ}} \frac{\partial\left(\tau_{y y}\right)}{\partial y}+\frac{1}{\rho_{\circ}} \frac{\partial\left(\tau_{y z}\right)}{\partial z}
\end{array}\right]_{i, j, k}^{n} \\
& +\Delta t\left[\theta g \cos \alpha \frac{\partial \eta}{\partial y}\right]_{i, j, k}^{n+1}
\end{aligned}
$$

Or, the above two equations could be written in term of $F_{x}$ and $F_{y}$ :

$$
\begin{aligned}
\bar{u}_{i, j, k}^{n+1} & =\bar{u}_{i, j, k}^{n}+\Delta t\left[F_{x}+(1-\theta) g \cos \alpha \frac{\partial \eta}{\partial x}-\frac{g \cos \alpha}{\rho_{\circ}} \int_{\eta}^{z} \frac{\partial \rho}{\partial x} d z+\frac{1}{\rho_{\circ}} \frac{\partial\left(\tau_{x z}\right)}{\partial z}\right]_{i, j, k}^{n} \\
& +\Delta t\left[\theta g \cos \alpha \frac{\partial \eta}{\partial x}\right]_{i, j, k}^{n+1} \\
\bar{v}_{i, j, k}^{n+1} & =\bar{v}_{i, j, k}^{n}+\Delta t\left[F_{y}+(1-\theta) g \cos \alpha \frac{\partial \eta}{\partial y}-\frac{g \cos \alpha}{\rho_{\circ}} \int_{\eta}^{z} \frac{\partial \rho}{\partial y} d z+\frac{1}{\rho_{\circ}} \frac{\partial\left(\tau_{y z}\right)}{\partial z}\right]_{i, j, k}^{n} \\
& +\Delta t\left[\theta g \cos \alpha \frac{\partial \eta}{\partial y}\right]_{i, j, k}^{n+1}
\end{aligned}
$$


Substituting $\bar{u}_{i, j, j}^{n+1}$ and $\bar{v}_{i, j, j}^{n+1}$ into free water surface equation for $\bar{u}$ and $\bar{v}$, respectively, the free water surface equation becomes,

$$
\begin{aligned}
\frac{\partial \eta}{\partial t} & =\frac{\partial}{\partial x} \int_{\eta}^{h} \bar{u}_{i, j, k}^{n} d z+\left.\Delta t \frac{\partial}{\partial x} \int_{\eta}^{h} F_{x}\right|_{i, j, k} ^{n} d z+\left.(1-\theta) \Delta t \frac{\partial}{\partial x} \int_{\eta}^{h} g \cos \alpha \frac{\partial \eta}{\partial x}\right|_{i, j} ^{n} d z \\
& -\left.\Delta t \frac{\partial}{\partial x} \int_{\eta}^{h} \frac{g \cos \alpha x}{\rho_{\circ}} \int_{\eta}^{z} \frac{\partial \rho}{\partial x} d z\right|_{i, j, k} ^{n} d z+\left.\Delta t \frac{\partial}{\partial x} \int_{\eta}^{h} \frac{1}{\rho_{\circ}} \frac{\partial\left(\tau_{x z}\right)}{\partial z}\right|_{i, j, k} ^{n} d z+\left.\theta \Delta t \frac{\partial}{\partial x} \int_{\eta}^{h} g \cos \alpha \frac{\partial \eta}{\partial x}\right|_{i, j} ^{n+1} d z \\
& +\frac{\partial}{\partial y} \int_{\eta}^{h} \bar{v}_{i, j, k}^{n} d z+\left.\Delta t \frac{\partial}{\partial y} \int_{\eta}^{h} F_{y}\right|_{i, j, k} ^{n} d z+\left.(1-\theta) \Delta t \frac{\partial}{\partial y} \int_{\eta}^{h} g \cos \alpha \frac{\partial \eta}{\partial y}\right|_{i, j} ^{n} d z \\
& -\left.\Delta t \frac{\partial}{\partial y} \int_{\eta}^{h} \frac{g \cos \alpha}{\rho_{\circ}} \int_{\eta}^{z} \frac{\partial \rho}{\partial y} d z\right|_{i, j, k} ^{n} d z+\left.\Delta t \frac{\partial}{\partial y} \int_{\eta}^{h} \frac{1}{\rho_{\circ}} \frac{\partial\left(\tau_{y z}\right)}{\partial z}\right|_{i, j, k} ^{n} d z+\theta \Delta t \frac{\partial}{\partial y} \int_{\eta}^{h} g \cos \alpha \frac{\partial \eta}{\left.\partial\right|_{i, j} ^{n+1}} d z
\end{aligned}
$$

The new terms are simplified as follows:

$$
\begin{aligned}
\left.(1-\theta) \Delta t \frac{\partial}{\partial x} \int_{\eta}^{h} g \cos \alpha \frac{\partial \eta}{\partial x}\right|_{i, j} ^{n} d z & =(1-\theta) \Delta \operatorname{tg} \cos \alpha \frac{\partial}{\partial x}\left[\frac{\partial \eta}{\partial x} \int_{\eta}^{h} d z\right]_{i, j}^{n} \\
& =\left.(1-\theta) \Delta \operatorname{tg} \cos \alpha \frac{\partial \eta}{\partial x}\right|_{i, j} ^{n} \frac{\partial}{\partial x} \int_{\eta}^{h} d z+\left.(1-\theta) \Delta \operatorname{tg} \cos \alpha \int_{\eta}^{h} d z \frac{\partial^{2} \eta}{\partial x^{2}}\right|_{i, j} ^{n} \\
& =(1-\theta) \Delta \operatorname{tg} \cos \alpha \frac{\eta_{i, j}^{n}-\eta_{i-1, j}^{n}}{\Delta x} \frac{\left.\int_{\eta}^{h} d z\right|_{i, j}-\left.\int_{\eta}^{h} d z\right|_{i-1, j}}{\Delta x} \\
& +\left.(1-\theta) \Delta \operatorname{tg} \cos \alpha \frac{\eta_{i+1, j}^{n}-2 \eta_{i, j}^{n}+\eta_{i-1, j}^{n}}{\Delta x^{2}} \int_{\eta}^{h} d z\right|_{i, j} \\
& =(1-\theta) \frac{\Delta \operatorname{tg} \cos \alpha}{\Delta x^{2}}\left[\left.\eta_{i-1, j}^{n} \sum_{k=1}^{K} H\right|_{i-1, j}-\eta_{i, j}^{n}\left(\left.\sum_{k=1}^{K} H\right|_{i, j}+\left.\sum_{k=1}^{K} H\right|_{i-1, j}\right)+\left.\eta_{i+1, j}^{n} \sum_{k=1}^{K} H\right|_{i, j}\right]
\end{aligned}
$$




$$
\begin{aligned}
\left.\theta \Delta t \frac{\partial}{\partial x} \int_{\eta}^{h} g \cos \alpha \frac{\partial \eta}{\partial x}\right|_{i, j} ^{n+1} d z & =\theta \Delta \operatorname{tg} \cos \alpha \frac{\partial}{\partial x}\left[\frac{\partial \eta}{\partial x} \int_{\eta}^{h} d z\right]_{i, j}^{n+1} \\
& =\left.\theta \Delta \operatorname{tg} \cos \alpha \frac{\partial \eta}{\partial x}\right|_{i, j} ^{n+1} \frac{\partial}{\partial x} \int_{\eta}^{h} d z+\left.\theta \Delta \operatorname{tg} \cos \alpha \int_{\eta}^{h} d z \frac{\partial^{2} \eta}{\partial x^{2}}\right|_{i, j} ^{n+1} \\
= & \theta \Delta \operatorname{tg} \cos \alpha \frac{\eta_{i, j}^{n+1}-\eta_{i-1, j}^{n+1}}{\Delta x} \frac{\left.\int_{\eta}^{h} d z\right|_{i, j}-\left.\int_{\eta}^{h} d z\right|_{i-1, j}}{\Delta x} \\
& +\left.\theta \Delta \operatorname{tg} \cos \alpha \frac{\eta_{i+1, j}^{n+1}-2 \eta_{i, j}^{n+1}+\eta_{i-1, j}^{n+1}}{\Delta x^{2}} \int_{\eta}^{h} d z\right|_{i, j} \\
= & \theta \frac{\Delta \operatorname{tg} \cos \alpha}{\Delta x^{2}}\left[\left.\eta_{i-1, j}^{n+1} \sum_{k=1}^{K} H\right|_{i-1, j}-\eta_{i, j}^{n+1}\left(\left.\sum_{k=1}^{K} H\right|_{i, j}+\left.\sum_{k=1}^{K} H\right|_{i-1, j}\right)+\left.\eta_{i+1, j}^{n+1} \sum_{k=1}^{K} H\right|_{i, j}\right]
\end{aligned}
$$

Same previous simplifications are applied to all other terms. Also, a forward difference is used for the unsteady term,

$$
\frac{\partial \eta}{\partial t}=\frac{\eta_{i, j}^{n+1}-\eta_{i, j}^{n}}{\Delta t}
$$

Substituting all the above terms in the free surface finite difference form, multiplying both sides by $\Delta t \Delta x \Delta y$, and re-arranging terms,

$$
\begin{aligned}
& {\left[\left.\frac{-\theta \Delta t^{2} \Delta y g \cos \alpha}{\Delta x} \sum_{k=1}^{K} H\right|_{i-1, j}\right] \eta_{i-1, j}^{n+1}+\left[\left.\frac{-\theta \Delta t^{2} \Delta x g \cos \alpha}{\Delta y} \sum_{k=1}^{K} H\right|_{i, j-1}\right] \eta_{i, j-1}^{n+1}+} \\
& {\left[\Delta x \Delta y+\frac{\theta \Delta t^{2} \Delta y g \cos \alpha}{\Delta x}\left(\left.\sum_{k=1}^{K} H\right|_{i, j}+\left.\sum_{k=1}^{K} H\right|_{i-1, j}\right)+\frac{\theta \Delta t^{2} \Delta x g \cos \alpha}{\Delta y}\left(\left.\sum_{k=1}^{K} H\right|_{i, j}+\left.\sum_{k=1}^{K} H\right|_{i, j-1}\right)\right] \eta_{i, j}^{n+1}+} \\
& {\left[\left.\frac{-\theta \Delta t^{2} \Delta x g \cos \alpha}{\Delta y} \sum_{k=1}^{K} H\right|_{i, j}\right] \eta_{i, j+1}^{n+1}+\left[\left.\frac{-\theta \Delta t^{2} \Delta y g \cos \alpha}{\Delta x} \sum_{k=1}^{K} H\right|_{i, j}\right] \eta_{i+1, j}^{n+1}=\Delta t \Delta x \Delta y[R H S]_{i, j, k}^{n}+\Delta x \Delta y \eta_{i, j}^{n}}
\end{aligned}
$$

Where: 


$$
\begin{aligned}
R H S & =\frac{1}{\Delta x}\left[\left.\sum_{k=1}^{K} \bar{u} H_{r x}\right|_{i, j}-\left.\sum_{k=1}^{K} \bar{u} H_{r x}\right|_{i-1, j}\right]+\frac{1}{\Delta y}\left[\left.\sum_{k=1}^{K} \bar{v} H_{r y}\right|_{i, j}-\left.\sum_{k=1}^{K} \bar{v} H_{r y}\right|_{i, j-1}\right] \\
& +\frac{\Delta t}{\Delta x}\left[\left.\sum_{k=1}^{K} F_{x} H_{r x}\right|_{i, j}-\left.\sum_{k=1}^{K} F_{x} H_{r x}\right|_{i-1, j}\right]+\frac{\Delta t}{\Delta y}\left[\left.\sum_{k=1}^{K} F_{y} H_{r y}\right|_{i, j}-\left.\sum_{k=1}^{K} F_{y} H_{r y}\right|_{i, j-1}\right] \\
& -\frac{\Delta t g \cos \alpha}{\rho_{\circ} \Delta x} \sum_{k=1}^{K}\left(\left.\sum_{k=1}^{K} \frac{\partial \rho}{\partial x} H^{2}\right|_{i, j}-\left.\sum_{k=1}^{K} \frac{\partial \rho}{\partial x} H^{2}\right|_{i-1, j}\right) \\
& -\frac{\Delta t g \cos \alpha}{\rho_{\circ} \Delta y} \sum_{k=1}^{K}\left(\left.\sum_{k=1}^{K} \frac{\partial \rho}{\partial y} H^{2}\right|_{i, j}-\left.\sum_{k=1}^{K} \frac{\partial \rho}{\partial y} H^{2}\right|_{i, j-1}\right) \\
& +\frac{\Delta t}{\rho_{\circ} \Delta x}\left[\left(\tau_{b x}-\tau_{s x}\right)_{i, j}-\left(\tau_{b x}-\tau_{s x}\right)_{i-1, j}\right]+\frac{\Delta t}{\rho_{\circ} \Delta y}\left[\left(\tau_{b y}-\tau_{s y}\right)_{i, j}-\left(\tau_{b y}-\tau_{s y}\right)_{i, j-1}\right] \\
& +\left[\left.(1-\theta) \frac{\Delta t g \cos \alpha}{\Delta x^{2}} \sum_{k=1}^{K} H\right|_{i-1, j}\right] \eta_{i-1, j}^{n}+\left[\left.(1-\theta) \frac{\Delta t g \cos \alpha}{\Delta y^{2}} \sum_{k=1}^{K} H\right|_{i, j-1}\right] \eta_{i, j-1}^{n} \\
& -\left[(1-\theta) \frac{\Delta t g \cos \alpha}{\Delta x^{2}}\left(\left.\sum_{k=1}^{K} H\right|_{i, j}+\left.\sum_{k=1}^{K} H\right|_{i-1, j}\right)+(1-\theta) \frac{\Delta t g \cos \alpha}{\Delta y^{2}}\left(\left.\sum_{k=1}^{K} H\right|_{i, j}+\left.\sum_{k=1}^{K} H\right|_{i, j-1}\right)\right] \eta_{i, j}^{n} \\
& +\left[\left.(1-\theta) \frac{\Delta t g \cos \alpha}{\Delta y^{2}} \sum_{k=1}^{K} H\right|_{i, j}\right] \eta_{i, j+1}^{n}+\left[\left.(1-\theta) \frac{\Delta t g \cos \alpha}{\Delta x^{2}} \sum_{k=1}^{K} H\right|_{i, j}\right] \eta_{i+1, j}^{n}
\end{aligned}
$$

For more simplification, the above equation can be written as follows:

$$
W \eta_{i-1, j}^{n+1}+N \eta_{i, j-1}^{n+1}+C \eta_{i, j}^{n+1}+S \eta_{i, j+1}^{n+1}+E \eta_{i+1, j}^{n+1}=R
$$

Where:

$$
\begin{aligned}
& W=\left[\left.\frac{-\theta \Delta t^{2} \Delta y g \cos \alpha}{\Delta x} \sum_{k=1}^{K} H\right|_{i-1, j}\right] \\
& N=\left[\left.\frac{-\theta \Delta t^{2} \Delta x g \cos \alpha}{\Delta y} \sum_{k=1}^{K} H\right|_{i, j-1}\right] \\
& C=\left[\Delta x \Delta y+\frac{\theta \Delta t^{2} \Delta y g \cos \alpha}{\Delta x}\left(\left.\sum_{k=1}^{K} H\right|_{i, j}+\left.\sum_{k=1}^{K} H\right|_{i-1, j}\right)+\frac{\theta \Delta t^{2} \Delta x g \cos \alpha}{\Delta y}\left(\left.\sum_{k=1}^{K} H\right|_{i, j}+\left.\sum_{k=1}^{K} H\right|_{i, j-1}\right)\right]
\end{aligned}
$$




$$
\begin{aligned}
& S=\left[\left.\frac{-\theta \Delta t^{2} \Delta x g \cos \alpha}{\Delta y} \sum_{k=1}^{K} H\right|_{i, j}\right] \\
& E=\left[\left.\frac{-\theta \Delta t^{2} \Delta y g \cos \alpha}{\Delta x} \sum_{k=1}^{K} H\right|_{i, j}\right] \\
& R=\Delta t \Delta y\left[\left.\sum_{k=1}^{K} \bar{u} H_{r x}\right|_{i, j}-\left.\sum_{k=1}^{K} \bar{u} H_{r x}\right|_{i-1, j}\right]+\Delta t \Delta x\left[\left.\sum_{k=1}^{K} \bar{v} H_{r y}\right|_{i, j}-\left.\sum_{k=1}^{K} \bar{v} H_{r y}\right|_{i, j-1}\right] \\
& +\Delta t^{2} \Delta y\left[\left.\sum_{k=1}^{K} F_{x} H_{r x}\right|_{i, j}-\left.\sum_{k=1}^{K} F_{x} H_{r x}\right|_{i-1, j}\right]+\Delta t^{2} \Delta x\left[\left.\sum_{k=1}^{K} F_{y} H_{r y}\right|_{i, j}-\left.\sum_{k=1}^{K} F_{y} H_{r y}\right|_{i, j-1}\right] \\
& -\frac{\Delta t^{2} \Delta y g \cos \alpha}{\rho_{\circ}} \sum_{k=1}^{K}\left(\left.\sum_{k=1}^{K} \frac{\partial \rho}{\partial x} H^{2}\right|_{i, j}-\left.\sum_{k=1}^{K} \frac{\partial \rho}{\partial x} H^{2}\right|_{i-1, j}\right) \\
& -\frac{\Delta t^{2} \Delta x g \cos \alpha}{\rho_{\circ}} \sum_{k=1}^{K}\left(\left.\sum_{k=1}^{K} \frac{\partial \rho}{\partial y} H^{2}\right|_{i, j}-\left.\sum_{k=1}^{K} \frac{\partial \rho}{\partial y} H^{2}\right|_{i, j-1}\right) \\
& +\frac{\Delta t^{2} \Delta y}{\rho_{\mathrm{o}}}\left[\left(\tau_{b x}-\tau_{s x}\right)_{i, j}-\left(\tau_{b x}-\tau_{s x}\right)_{i-1, j}\right]+\frac{\Delta t^{2} \Delta x}{\rho_{\mathrm{o}}}\left[\left(\tau_{b y}-\tau_{s y}\right)_{i, j}-\left(\tau_{b y}-\tau_{s y}\right)_{i, j-1}\right] \\
& +\left[\left.(1-\theta) \frac{\Delta t^{2} \Delta y g \cos \alpha}{\Delta x} \sum_{k=1}^{K} H\right|_{i-1, j}\right] \eta_{i-1, j}^{n}+\left[\left.(1-\theta) \frac{\Delta t^{2} \Delta x g \cos \alpha}{\Delta y} \sum_{k=1}^{K} H\right|_{i, j-1}\right] \eta_{i, j-1}^{n} \\
& +\left[\begin{array}{l}
\Delta x \Delta y-(1-\theta) \frac{\Delta t^{2} \Delta y g \cos \alpha}{\Delta x}\left(\left.\sum_{k=1}^{K} H\right|_{i, j}+\left.\sum_{k=1}^{K} H\right|_{i-1, j}\right) \\
-(1-\theta) \frac{\Delta t^{2} \Delta x g \cos \alpha}{\Delta y}\left(\left.\sum_{k=1}^{K} H\right|_{i, j}+\left.\sum_{k=1}^{K} H\right|_{i, j-1}\right)
\end{array}\right] \eta_{i, j}^{n} \\
& +\left[\left.(1-\theta) \frac{\Delta t^{2} \Delta x g \cos \alpha}{\Delta y} \sum_{k=1}^{K} H\right|_{i, j}\right] \eta_{i, j+1}^{n}+\left[\left.(1-\theta) \frac{\Delta t^{2} \Delta y g \cos \alpha}{\Delta x} \sum_{k=1}^{K} H\right|_{i, j}\right] \eta_{i+1, j}^{n}
\end{aligned}
$$

For $\theta=1$, the scheme becomes fully implicit and reverts to what we did previously for the numerical solution of the three-dimensional equations of the free surface and momentums without inclusion the degree of implicitness, but known water levels in the final solution of the free surface equation were taken from the previous time level $n$ in 
addition to treat the free surface elevation at the level $n$ implicitly in the solution of the momentum equations.

The solution of the X-Momentum equation is also by the splitting method:

Stage 1:

$$
\begin{aligned}
\frac{\partial \bar{u}}{\partial t} & =-\bar{u} \frac{\partial \bar{u}}{\partial x}-\bar{v} \frac{\partial \bar{u}}{\partial y}-\bar{w} \frac{\partial \bar{u}}{\partial z}+g \cos \alpha \frac{\partial \eta}{\partial x}-\frac{g \cos \alpha}{\rho_{\circ}} \int_{\eta}^{z} \frac{\partial \rho}{\partial x} d z+f \bar{v}+g \sin \alpha \\
& +\frac{1}{\rho_{\circ}} \frac{\partial\left(\tau_{x x}\right)}{\partial x}+\frac{1}{\rho_{\circ}} \frac{\partial\left(\tau_{x y}\right)}{\partial y}+\frac{1}{\rho_{\circ}} \frac{\partial\left(\tau_{s x}+\tau_{b x}\right)}{\partial z}
\end{aligned}
$$

Stage 2:

$$
\frac{\partial \bar{u}}{\partial t}=\frac{1}{\rho_{\circ}} \frac{\partial\left(\tau_{x z}\right)}{\partial z}
$$

In the first stage,

$$
\begin{aligned}
\bar{u}_{i, j, k}^{*}= & \bar{u}_{i, j, k}^{n}+\Delta t\left[\begin{array}{l}
-\bar{u} \frac{\partial \bar{u}}{\partial x}-\bar{v} \frac{\partial \bar{u}}{\partial y}-\bar{w} \frac{\partial \bar{u}}{\partial z}+(1-\theta) g \cos \alpha \frac{\partial \eta}{\partial x}-\frac{g \cos \alpha}{\rho_{\circ}} \int_{\eta}^{z} \frac{\partial \rho}{\partial x} d z+f \bar{v} \\
+g \sin \alpha+\frac{1}{\rho_{\circ}} \frac{\partial\left(\tau_{x x}\right)}{\partial x}+\frac{1}{\rho_{\circ}} \frac{\partial\left(\tau_{x y}\right)}{\partial y}+\frac{1}{\rho_{\circ}} \frac{\partial\left(\tau_{s x}+\tau_{b x}\right)}{\partial z}
\end{array}\right]_{i, j, k}^{n} \\
+ & +\left[\Delta t \theta g \cos \alpha \frac{\partial \eta}{\partial x}\right]_{i, j, k}^{n+1}
\end{aligned}
$$

The new terms are simplified as follows:

$$
\begin{aligned}
\left.(1-\theta) g \cos \alpha \frac{\partial \eta}{\partial x}\right|_{i, j, k} ^{n}-\left.\frac{g \cos \alpha}{\rho_{\circ}} \int_{\eta}^{z} \frac{\partial \rho}{\partial x} d z\right|_{i, j, k} ^{n} & =(1-\theta) \frac{g \cos \alpha}{\Delta x}\left(\eta_{i+1, j, k}^{n}-\eta_{i, j, k}^{n}\right) \\
& -\left.\frac{g \cos \alpha}{\rho \Delta x} \sum_{k=1}^{K}\left(\rho_{i+1, j, k}^{n}-\rho_{i, j, k}^{n}\right) H_{r x}\right|_{i, j, k} ^{n}
\end{aligned}
$$


$\left.\theta g \cos \alpha \frac{\partial \eta}{\partial x}\right|_{i, j, k} ^{n+1}=\theta \frac{g \cos \alpha}{\Delta x}\left(\eta_{i+1, j, k}^{n+1}-\eta_{i, j, k}^{n+1}\right)$

By collecting all the terms, the final finite difference equation of $\bar{u}_{i, j, k}^{*}$ will be as follows:

$$
\begin{aligned}
\bar{u}_{i, j, k}^{*}= & \bar{u}_{i, j, k}^{n}-\frac{\Delta t}{\Delta x}\left[\bar{u}_{i, j, k}\left[\left[\bar{u}_{i, j, k}, 0\right]\right]-\bar{u}_{i, j, k}\left(\left(\bar{u}_{i, j, k}, 0\right)\right)-\bar{u}_{i-1, j, k}\left[\left[\bar{u}_{i, j, k}, 0\right]\right]+\bar{u}_{i+1, j, k}\left(\left(\bar{u}_{i, j, k}, 0\right)\right)\right]^{n} \\
& -\frac{\Delta t}{\Delta y}\left[\bar{u}_{i, j, k}\left[\left[\bar{v}_{i, j, k}, 0\right]\right]-\bar{u}_{i, j, k}\left(\left(\bar{v}_{i, j, k}, 0\right)\right)-\bar{u}_{i, j-1, k}\left[\left[\bar{v}_{i, j, k}, 0\right]\right]+\bar{u}_{i, j+1, k}\left(\left(\bar{v}_{i, j, k}, 0\right)\right)\right]^{n} \\
& -\frac{\Delta t}{\Delta z}\left[\bar{u}_{i, j, k}\left[\left[\bar{w}_{i, j, k}, 0\right]\right]-\bar{u}_{i, j, k}\left(\left(\bar{w}_{i, j, k}, 0\right)\right)-\bar{u}_{i, j-1, k}\left[\left[\bar{w}_{i, j, k}, 0\right]\right]+\bar{u}_{i, j+1, k}\left(\left(\bar{w}_{i, j, k}, 0\right)\right)\right]^{n} \\
& +(1-\theta) \frac{\Delta t g \cos \alpha}{\Delta x}\left(\eta_{i+1, j, k}^{n}-\eta_{i, j, k}^{n}\right)+\theta \frac{g \cos \alpha}{\Delta x}\left(\eta_{i+1, j, k}^{n+1}-\eta_{i, j, k}^{n+1}\right) \\
& -\left.\frac{\Delta t g \cos \alpha}{\rho \Delta x} \sum_{k=1}^{K}\left(\rho_{i+1, j, k}^{n}-\rho_{i, j, k}^{n}\right) H_{r x}\right|_{i, j, k} ^{n}+\Delta t f \bar{v}_{i, j, k}^{n}+\Delta t g \sin \alpha \\
& +\frac{\Delta t v_{h}}{\Delta x_{i, j, k}}\left[\frac{\bar{u}_{i+1, j, k}-\bar{u}_{i, j, k}}{\Delta x_{i+1 / 2, j, k}}-\frac{\bar{u}_{i, j, k}-\bar{u}_{i-1, j, k}}{\Delta x_{i-1 / 2, j, k}}\right]^{n}+\frac{\Delta t v_{h}}{\Delta y_{i, j, k}}\left[\frac{\bar{u}_{i, j+1, k}-\bar{u}_{i, j, k}}{\Delta y_{i, j+1 / 2, k}}-\frac{\bar{u}_{i, j, k}-\bar{u}_{i, j-1, k}}{\Delta y_{i, j-1 / 2, k}}\right]^{n} \\
& +\frac{\Delta t}{\rho_{o} \Delta z}\left[\left(\tau_{s x}+\tau_{b x}\right)_{i, j, k+1 / 2}-\left(\tau_{s x}+\tau_{b x}\right)_{i, j, k-1 / 2}\right]^{h}
\end{aligned}
$$

Then, $\bar{u}_{i, j, k}^{*}$ is calculated at each cell face and will be used to calculate $\bar{u}_{i, j, k}^{n+1}$ by solving the second stage implicitly, see the previous solution of the fully implicit scheme (Section 2.5.4).

A similar formulation for the numerical solution of the Y-Momentum equation leads to: 


$$
\begin{aligned}
\bar{v}_{i, j, k}^{*}= & \bar{v}_{i, j, k}^{n}-\frac{\Delta t}{\Delta x}\left[\bar{v}_{i, j, k}\left[\left[\bar{u}_{i, j, k}, 0\right]\right]-\bar{v}_{i, j, k}\left(\left(\bar{u}_{i, j, k}, 0\right)\right)-\bar{v}_{i-1, j, k}\left[\left[\bar{u}_{i, j, k}, 0\right]\right]+\bar{v}_{i+1, j, k}\left(\left(\bar{u}_{i, j, k}, 0\right)\right)\right]^{n} \\
& -\frac{\Delta t}{\Delta y}\left[\bar{v}_{i, j, k}\left[\left[\bar{v}_{i, j, k}, 0\right]\right]-\bar{v}_{i, j, k}\left(\left(\bar{v}_{i, j, k}, 0\right)\right)-\bar{v}_{i, j-1, k}\left[\left[\bar{v}_{i, j, k}, 0\right]\right]+\bar{v}_{i, j+1, k}\left(\left(\bar{v}_{i, j, k}, 0\right)\right)\right]^{n} \\
& -\frac{\Delta t}{\Delta z}\left[\bar{v}_{i, j, k}\left[\left[\bar{w}_{i, j, k}, 0\right]\right]-\bar{v}_{i, j, k}\left(\left(\bar{w}_{i, j, k}, 0\right)\right)-\bar{v}_{i, j-1, k}\left[\left[\bar{w}_{i, j, k}, 0\right]\right]+\bar{v}_{i, j+1, k}\left(\left(\bar{w}_{i, j, k}, 0\right)\right)\right]^{n} \\
& +(1-\theta) \frac{\Delta t g \cos \alpha}{\Delta y}\left(\eta_{i, j+1, k}^{n}-\eta_{i, j, k}^{n}\right)+\theta \frac{\Delta t g \cos \alpha}{\Delta y}\left(\eta_{i, j+1, k}^{n+1}-\eta_{i, j, k}^{n+1}\right) \\
& -\left.\frac{\Delta t g \cos \alpha}{\rho \Delta y} \sum_{k=1}^{K}\left(\rho_{i, j+1, k}^{n}-\rho_{i, j, k}^{n}\right) H_{r y}\right|_{i, j, k} ^{n}-\Delta t f \bar{u}_{i, j, k}^{n} \\
& +\frac{\Delta t v_{h}}{\Delta x_{i, j, k}}\left[\frac{\bar{v}_{i+1, j, k}-\bar{v}_{i, j, k}}{\Delta x_{i+1 / 2, j, k}}-\frac{\bar{v}_{i, j, k}-\bar{v}_{i-1, j, k}}{\Delta x_{i-1 / 2, j, k}}\right]^{n}+\frac{\Delta t v_{h}}{\Delta y_{i, j, k}}\left[\frac{\bar{v}_{i, j+1, k}-\bar{v}_{i, j, k}}{\Delta y_{i, j+1 / 2, k}}-\frac{\bar{v}_{i, j, k}-\bar{v}_{i, j-1, k}}{\Delta y_{i, j-1 / 2, k}}\right]^{n} \\
& +\frac{\Delta t}{\rho_{\circ} \Delta z}\left[\left(\tau_{s y}+\tau_{b y}\right)_{i, j, k+1 / 2}-\left(\tau_{s y}+\tau_{b y}\right)_{i, j, k-1 / 2}\right.
\end{aligned}
$$

Then, $\bar{v}_{i, j, k}^{*}$ is used to calculate $\bar{v}_{i, j, k}^{n+1}$ implicitly in the second stage, see the previous solution of the fully implicit scheme (Section 2.5.4).

\subsubsection{Numerical Solution of the Continuity Equation}

After calculating $\bar{u}_{i, j, k}^{n+1}$ and $\bar{v}_{i, j, k}^{n+1}$, the vertical velocity component $\bar{w}_{i, j, k}^{n+1}$ can be calculated from the continuity equation by implementing the cell by cell calculations and as follows:

$$
\begin{gathered}
\frac{\partial \bar{u}}{\partial x}+\frac{\partial \bar{v}}{\partial y}+\frac{\partial \bar{w}}{\partial z}=0 \\
\frac{\bar{u}_{i, j, k}^{n+1}-\bar{u}_{i-1, j, k}^{n+1}}{\Delta x}+\frac{\bar{v}_{i, j, k}^{n+1}-\bar{v}_{i, j-1, k}^{n+1}}{\Delta y}+\frac{\bar{w}_{i, j, k}^{n+1}-\bar{w}_{i, j, k-1}^{n+1}}{\Delta z}=0 \\
\bar{w}_{i, j, k-1}^{n+1}=\bar{w}_{i, j, k}^{n+1}+\frac{\bar{u}_{i, j, k}^{n+1}-\bar{u}_{i-1, j, k}^{n+1}}{\Delta x} \Delta z+\frac{\bar{v}_{i, j, k}^{n+1}-\bar{v}_{i, j-1, k}^{n+1}}{\Delta y} \Delta z
\end{gathered}
$$


The solution starts from the bottom of the waterbody where the vertical velocity components are known by the boundary condition $\bar{w}_{i, j, k b(i, j)}=0$.

\subsubsection{Numerical Treatment of the Total Waterbody Height}

Now, the horizontal and vertical velocities are known at the new time level $(n+1)$. Then, same calculations need to be done to get the free surface elevation and velocities at the next level and so forth. At every time level, the surface layer thickness is updated because the surface grid cells have variable depth with time unlike the cells below. The depth of the surface cell at any time level equals to the summation of the depth increment, which is constant for every cell, and free surface elevation at that level, this can be represented in a general form as follows:

$$
H_{i, j, k t}^{n}=\Delta z_{i, j, k t}-\eta_{i, j}^{n}
$$

In the above equation, it is clear that if $\eta_{i, j}^{n}$ is positive, $H_{i, j, k t}^{n}$ will be less than $\Delta z$, and if it is negative, $H_{i, j, k t}^{n}$ will be greater than $\Delta z$.

Thus, the total waterbody height at any time level is

$$
D_{i, j}^{n}=\sum_{k=k t+1}^{k b(i, j)} H_{i, j, k}+H_{i, j, k t}^{n}
$$

Where: $D_{i, j}^{n}$ is the total waterbody height at the level $(n)$.

\subsubsection{Numerical Solution of Heat and Water Quality Transport Equation}

The numerical solution of the transport equation was formulated by splitting the model time step. Also, this derivation could be done without splitting the time step as follows: 
Formulation by splitting the model time step: The heat and water quality transport equation is split into two equations in two stages at the same model time step. In the first stage, the equation is treated explicitly by implementing the first-order upwind scheme and higher-order schemes for the advective terms. In the second equation, the equation is treated implicitly by implementing a fully implicit scheme for the vertical diffusion term:

$$
\frac{\partial \phi}{\partial t}+\frac{\partial \bar{u} \phi}{\partial x}+\frac{\partial \bar{v} \phi}{\partial y}+\frac{\partial \bar{w} \phi}{\partial z}=\frac{\partial}{\partial x}\left[D_{x} \frac{\partial \phi}{\partial x}\right]+\frac{\partial}{\partial y}\left[D_{y} \frac{\partial \phi}{\partial y}\right]+\frac{\partial}{\partial z}\left[D_{z} \frac{\partial \phi}{\partial z}\right]+S_{\phi}
$$

Stage 1:

$$
\frac{\partial \phi}{\partial t}+\frac{\partial \bar{u} \phi}{\partial x}+\frac{\partial \bar{\nu} \phi}{\partial y}+\frac{\partial \bar{w} \phi}{\partial z}=\frac{\partial}{\partial x}\left[D_{x} \frac{\partial \phi}{\partial x}\right]+\frac{\partial}{\partial y}\left[D_{y} \frac{\partial \phi}{\partial y}\right]+S_{\phi}
$$

Stage 2:

$$
\frac{\partial \phi}{\partial t}=\frac{\partial}{\partial z}\left[D_{z} \frac{\partial \phi}{\partial z}\right]
$$

The finite difference formulation of the first stage is as follows by using the first-order upwind scheme for advective terms and discretizing the horizontal diffusion terms and source/sink term explicitly.

$$
\begin{aligned}
\left.a d v\right|_{i, j, k} ^{n} & =\frac{1}{\Delta x_{i, j, k}}\left[\phi_{i, j, k}\left[\left[\bar{u}_{i, j, k}, 0\right]\right]+\phi_{i+1, j, k}\left(\left(\bar{u}_{i, j, k}, 0\right)\right)-\phi_{i-1, j, k}\left[\left[\bar{u}_{i-1, j, k}, 0\right]\right]-\phi_{i, j, k}\left(\left(\bar{u}_{i-1, j, k}, 0\right)\right)\right]^{n} \\
& +\frac{1}{\Delta y_{i, j, k}}\left[\phi_{i, j, k}\left[\left[\bar{v}_{i, j, k}, 0\right]\right]+\phi_{i, j+1, k}\left(\left(\bar{v}_{i, j, k}, 0\right)\right)-\phi_{i, j-1, k}\left[\left[\bar{v}_{i, j-1, k}, 0\right]\right]-\phi_{i, j, k}\left(\left(\bar{v}_{i, j-1, k}, 0\right)\right)\right]^{n} \\
& +\frac{1}{\Delta z_{i, j, k}}\left[\phi_{i, j, k}\left[\left[\bar{w}_{i, j, k}, 0\right]\right]+\phi_{i, j, k+1}\left(\left(\bar{w}_{i, j, k}, 0\right)\right)-\phi_{i, j, k-1}\left[\left[\bar{w}_{i, j, k-1}, 0\right]\right]-\phi_{i, j, k}\left(\left(\bar{w}_{i, j, k-1}, 0\right)\right)\right]^{n}
\end{aligned}
$$




$$
\left.\operatorname{diff}\right|_{i, j, k} ^{n}=\frac{D_{x}}{\Delta x_{i, j, k}}\left[\frac{\phi_{i+1, j, k}-\phi_{i, j, k}}{\Delta x_{i+1 / 2, j, k}}-\frac{\phi_{i, j, k}-\phi_{i-1, j, k}}{\Delta x_{i-1 / 2, j, k}}\right]^{n}+\frac{D_{y}}{\Delta y_{i, j, k}}\left[\frac{\phi_{i, j+1, k}-\phi_{i, j, k}}{\Delta y_{i, j+1 / 2, k}}-\frac{\phi_{i, j, k}-\phi_{i, j-1, k}}{\Delta y_{i, j-1 / 2, k}}\right]^{n}
$$

Then,

$$
\left.\phi\right|_{i, j, k} ^{*}=\left.\phi\right|_{i, j, k} ^{n}+\Delta t\left(-\left.a d v\right|_{i, j, k} ^{n}+\left.\operatorname{diff}\right|_{i, j, k} ^{n}+\left.S_{\phi}\right|_{i, j, k} ^{n}\right)
$$

The finite difference formulation of the second stage is as follows:

$$
\begin{aligned}
& \frac{\left.\phi\right|_{i, j, k} ^{n+1}-\left.\phi\right|_{i, j, k} ^{*}}{\Delta t}=\frac{1}{\Delta z_{i, j, k}}\left[D_{z i, j, k} \frac{\phi_{i, j, k+1}-\phi_{i, j, k}}{\Delta z_{i, j, k+1 / 2}}-D_{z i, j, k-1} \frac{\phi_{i, j, k}-\phi_{i, j, k-1}}{\Delta z_{i, j, k-1 / 2}}\right]^{n+1} \\
& {\left[-\frac{\Delta t D_{z i, j, k-1}}{\Delta z_{i, j, k} \Delta z_{i, j, k-1 / 2}}\right] \phi_{i, j, k-1}^{n+1}+\left[1+\frac{\Delta t D_{z i, j, k}}{\Delta z_{i, j, k} \Delta z_{i, j, k+1 / 2}}+\frac{\Delta t D_{z i, j, k-1}}{\Delta z_{i, j, k} \Delta z_{i, j, k-1 / 2}}\right] \phi_{i, j, k}^{n+1}} \\
& +\left[-\frac{\Delta t D_{z i, j, k}}{\Delta z_{i, j, k} \Delta z_{i, j, k+1 / 2}}\right] \phi_{i, j, k+1}^{n+1}=\phi_{i, j, k}^{*}
\end{aligned}
$$

For more simplification, the above equation can be written as follows:

$$
U \phi_{i, j, k-1}^{n+1}+C \phi_{i, j, k}^{n+1}+D \phi_{i, j, k+1}^{n+1}=R
$$

Where:

$$
\begin{aligned}
& U=\left[-\frac{\Delta t D_{v i, j, k-1}}{\Delta z_{i, j, k} \Delta z_{i, j, k-1 / 2}}\right] \\
& C=\left[1+\frac{\Delta t D_{v i, j, k}}{\Delta z_{i, j, k} \Delta z_{i, j, k+1 / 2}}+\frac{\Delta t D_{v i, j, k-1}}{\Delta z_{i, j, k} \Delta z_{i, j, k-1 / 2}}\right] \\
& D=\left[-\frac{\Delta t D_{v i, j, k}}{\Delta z_{i, j, k} \Delta z_{i, j, k+1 / 2}}\right] \\
& R=\phi_{i, j, k}^{*}
\end{aligned}
$$


Formulation without splitting the model time step: The heat and water quality transport equation is transformed to finite difference form by implementing the same previous formulation, but the finite difference discretization is performed at the same model time step.

$$
\begin{aligned}
\frac{\left.\phi\right|_{i, j, k} ^{n+1}-\left.\phi\right|_{i, j, k} ^{n}}{\Delta t}= & -\left.a d v\right|_{i, j, k} ^{n}+\left.\operatorname{diff}\right|_{i, j, k} ^{n}+\frac{1}{\Delta z_{i, j, k}}\left[D_{z i, j, k} \frac{\phi_{i, j, k+1}-\phi_{i, j, k}}{\Delta z_{i, j, k+1 / 2}}-D_{z i, j, k-1} \frac{\phi_{i, j, k}-\phi_{i, j, k-1}}{\Delta z_{i, j, k-1 / 2}}\right]^{n+1} \\
& +\left.S_{\phi}\right|_{i, j, k} ^{n}
\end{aligned}
$$

For more simplifications,

$$
\begin{aligned}
\left.\phi\right|_{i, j, k} ^{n+1}= & \left.\phi\right|_{i, j, k} ^{n}-\left.\Delta t * a d v\right|_{i, j, k} ^{n}+\left.\Delta t * d i f f\right|_{i, j, k} ^{n}+\left.\Delta t * S_{\phi}\right|_{i, j, k} ^{n} \\
& +\frac{\Delta t}{\Delta z_{i, j, k}}\left[D_{z i, j, k} \frac{\phi_{i, j, k+1}-\phi_{i, j, k}}{\Delta z_{i, j, k+1 / 2}}-D_{z i, j, k-1} \frac{\phi_{i, j, k}-\phi_{i, j, k-1}}{\Delta z_{i, j, k-1 / 2}}\right]^{n+1} \\
& \quad\left[-\frac{\Delta t D_{z i, j, k-1}}{\Delta z_{i, j, k} \Delta z_{i, j, k-1 / 2}}\right] \phi_{i, j, k-1}^{n+1}+\left[1+\frac{\Delta t D_{z i, j, k}}{\Delta z_{i, j, k} \Delta z_{i, j, k+1 / 2}}+\frac{\Delta t D_{z i, j, k-1}}{\Delta z_{i, j, k} \Delta z_{i, j, k-1 / 2}}\right] \phi_{i, j, k}^{n+1} \\
& +\left[-\frac{\Delta t D_{z i, j, k}}{\Delta z_{i, j, k} \Delta z_{i, j, k+1 / 2}}\right] \phi_{i, j, k+1}^{n+1}=R_{i, j, k}^{n}
\end{aligned}
$$

Rearranging the terms,

$$
U \phi_{i, j, k-1}^{n+1}+C \phi_{i, j, k}^{n+1}+D \phi_{i, j, k+1}^{n+1}=R_{i, j, k}^{n}
$$

Where:

$$
U=\left[-\frac{\Delta t D_{v i, j, k-1}}{\Delta z_{i, j, k} \Delta z_{i, j, k-1 / 2}}\right]
$$




$$
\begin{aligned}
& C=\left[1+\frac{\Delta t D_{v i, j, k}}{\Delta z_{i, j, k} \Delta z_{i, j, k+1 / 2}}+\frac{\Delta t D_{v i, j, k-1}}{\Delta z_{i, j, k} \Delta z_{i, j, k-1 / 2}}\right] \\
& D=\left[-\frac{\Delta t D_{v i, j, k}}{\Delta z_{i, j, k} \Delta z_{i, j, k+1 / 2}}\right] \\
& R_{i, j, k}^{n}=\left.\phi\right|_{i, j, k} ^{n}-\left.\Delta t^{*} a d v\right|_{i, j, k} ^{n}+\left.\Delta t * d i f f\right|_{i, j, k} ^{n}+\left.\Delta t * S_{\phi}\right|_{i, j, k} ^{n}
\end{aligned}
$$

The above formulation of advective terms of the heat and water quality transport equation was based on first-order UPWIND scheme finite differencing, and it accounts for positive and negative flow. However, the first-order UPWIND scheme results in a numerical diffusion, distorted the numerical solution primarily by dissipation error, due to the even spatial derivative in the truncation error of the discretization. For a simple numerical solution of the advective problem such as that is presented in the one way wave equation, the numerical diffusion can easily be determined by using Tylor Series Expansions and it has an order of magnitude of $\left[0.5 \bar{u} \Delta x-0.5 \bar{u}^{2} \Delta t\right]$ for the $x$-direction advection, $\left[0.5 \bar{v} \Delta y-0.5 \bar{v}^{2} \Delta t\right]$ for the $y$-direction advection, or $\left[0.5 \bar{w} \Delta z-0.5 \bar{w}^{2} \Delta t\right]$ for the $z-$ direction advection. The presence of the numerical diffusion associated with the firstorder UPWIND scheme does not reflect the processes of advective properly and accurately. This problem arises clearly in sharp front regions in which there is a concentration jump or a concentration discontinuity such as at the thermocline level during the stratification period in lakes and reservoirs. Therefore, using the first-order UPWIND scheme yields a smoothly varying curve shape at the sharp front edges. Thus, in order to reduce the amount of the numerical diffusion resulting from the first-order 
UPWIND, higher-order schemes (QUICK and QUICKEST) have been recommended for modeling the advective or convective transport processes (Cole \& Wells, 2017; Edinger, 2001; Kowalik \& Murty, 1993; Leonard, 1979, 1991; Neumann, Simunek, \& Cook, 2011). However, problems associated with implementing these schemes are higher computational cost, storage, and programing difficulties.

Leonard (1979) developed the QUICK (Quadratic Upstream Interpolation for Convective Kinematics) and QUICKEST (Quadratic Upstream Interpolation for Convective Kinematics with Estimated Upstream Terms) scheme, an explicit method to solve the advective terms of the transport equation by using quadratic upstream interpolation. For the case when both advective and diffusion processes exist and the advection is high, the QUICK method is a third-order accurate in space and QUICKEST method is a thirdorder accurate in space and time. Also, whereas the OUICK scheme is appropriate to steady or quasi-steady flow when there is high advective in one-dimension, the QUICKEST scheme is suitable for the unsteady flow (Leonard, 1979).

Because the advective terms of the horizontal momentum equations have a small contribution compared with the shear or pressure terms, Quadratic Upstream Interpolation methods are not recommended for modeling the advective terms of the hydrodynamic part of the governing equations since these methods may lead to a low accurate solution in such a case. However, in the transport equation the advection is dominant horizontally, while the diffusion is dominant vertically. Thus, implementing QUICK and QUICKEST method is essential (Leonard, 1979). 
Buchak and Cole (1995) have been implementing the QUICKEST algorithm in CEQUAL-W2 for the explicit advective transport longitudinally and vertically. As a result, using the QUICKEST scheme makes the overall accuracy higher. In addition, Cole and Wells (2017) integrated the QUICKEST scheme in CE-QUAL-W2 by using the ULTIMATE scheme (Universal Limiter for Transient Interpolation Modeling of the Advective Transport Equations) (Leonard, 1991) to eliminate the over and undershoots that are associated with the QUICKEST scheme near a gradient.

The present 3D model calculates the constituent $\phi$ at the grid interfaces based on the conservative transport equation as follows:

$$
\begin{aligned}
\left.a d v\right|_{i, j, k} ^{n}= & \frac{1}{\Delta x_{i, j, k}}\left[\phi_{i+1 / 2, j, k}\left[\left[\bar{u}_{i, j, k}, 0\right]\right]+\phi_{i+1 / 2, j, k}\left(\left(\bar{u}_{i, j, k}, 0\right)\right)-\phi_{i-1 / 2, j, k}\left[\left[\bar{u}_{i-1, j, k}, 0\right]\right]-\phi_{i-1 / 2, j, k}\left(\left(\bar{u}_{i-1, j, k}, 0\right)\right)\right]^{n} \\
& +\frac{1}{\Delta y_{i, j, k}}\left[\phi_{i, j+1 / 2, k}\left[\left[\bar{v}_{i, j, k}, 0\right]\right]+\phi_{i, j+1 / 2, k}\left(\left(\bar{v}_{i, j, k}, 0\right)\right)-\phi_{i, j-1 / 2, k}\left[\left[\bar{v}_{i, j-1, k}, 0\right]\right]-\phi_{i, j-1 / 2, k}\left(\left(\bar{v}_{i, j-1, k}, 0\right)\right)\right]^{n} \\
& +\frac{1}{\Delta z_{i, j, k}}\left[\phi_{i, j, k+1 / 2}\left[\left[\bar{w}_{i, j, k}, 0\right]\right]+\phi_{i, j, k+1 / 2}\left(\left(\bar{w}_{i, j, k}, 0\right)\right)-\phi_{i, j, k-1 / 2}\left[\left[\bar{w}_{i, j, k-1}, 0\right]\right]-\phi_{i, j, k-1 / 2}\left(\left(\bar{w}_{i, j, k-1}, 0\right)\right)\right]^{h}
\end{aligned}
$$

Where $\phi_{i-1 / 2}^{n}$ and $\phi_{i+1 / 2}^{n}$ are the constituent value at the grid interfaces in which the face value that is next to $[[a, b]]$ term is taken under the presence of the positive flow, and the value that is next to $((a, b))$ term is taken under the presence of the negative flow. The $\phi_{i-1 / 2}^{n}$ and $\phi_{i+1 / 2}^{n}$ values are calculated by implementing the QUICK, QUICKEST, or ULTIMATE QUICKEST scheme as follows: 


\section{The QUICK scheme:}

The QUICK scheme estimates the constituent $\phi$ at the grid interfaces by using the Quadratic Upstream Interpolation. For positive flow and constant grid spacing, the $x$ direction constituent advective at the node $(i)$ is

$$
\left.\frac{\partial u \phi}{\partial x}\right|_{i} ^{n}=\frac{\phi_{i+1 / 2}^{n}\left[\left[u_{i}^{n}, 0\right]\right]-\phi_{i-1 / 2}^{n}\left[\left[u_{i-1}^{n}, 0\right]\right]}{\Delta x}
$$

Where $\phi_{i-1 / 2}^{n}$ and $\phi_{i+1 / 2}^{n}$ are determined by applying the quadratic interpolation polynomial along the uniform grid spacing. The quadratic polynomial formula as follows:

$$
\phi=\phi_{0}+\phi_{1} x+\phi_{2} x^{2}
$$

Where $\phi_{0}, \phi_{1}$, and $\phi_{2}$ are constants and $x$ is a local coordinate at the node $(i-1)$.

By substituting the constituent values at the nodes $(i-2, i-1$, and $i)$ in the quadratic polynomial formula with the corresponding distance from the local coordinate origin $\left(-\Delta x, 0\right.$, and $\Delta x$, respectively), we get three linear equations in term of $\left(\phi_{0}, \phi_{1}\right.$, and $\left.\phi_{2}\right)$. Solving for the constants $\left(\phi_{0}, \phi_{1}\right.$, and $\left.\phi_{2}\right)$ leads to formulate the local quadratic polynomial equation which can be easily used to estimate the constituent values at the left interface, $\phi_{i-1 / 2}^{n}$ :

$$
\phi_{i-1 / 2}^{n}=\frac{1}{2}\left(\phi_{i-1}^{n}+\phi_{i}^{n}\right)-\frac{1}{8}\left(\phi_{i-2}^{n}-2 \phi_{i-1}^{n}+\phi_{i}^{n}\right)
$$

Changing the local coordinate to be at the node $(i)$ and using the nodes ( $i-1, i$, and $i+1$ ), the derivation ends with the constituent values at the right interface, $\phi_{i+1 / 2}^{n}$ :

$$
\phi_{i+1 / 2}^{n}=\frac{1}{2}\left(\phi_{i}^{n}+\phi_{i+1}^{n}\right)-\frac{1}{8}\left(\phi_{i-1}^{n}-2 \phi_{i}^{n}+\phi_{i+1}^{n}\right)
$$


Similar steps should be done for the negative flow based on the nodes $(i-1, i, i+1$, $i+2)$. The constituent values at the left and right interface become:

$$
\begin{aligned}
& \phi_{i-1 / 2}^{n}=\frac{1}{2}\left(\phi_{i-1}^{n}+\phi_{i}^{n}\right)-\frac{1}{8}\left(\phi_{i-1}^{n}-2 \phi_{i}^{n}+\phi_{i+1}^{n}\right) \\
& \phi_{i+1 / 2}^{n}=\frac{1}{2}\left(\phi_{i}^{n}+\phi_{i+1}^{n}\right)-\frac{1}{8}\left(\phi_{i}^{n}-2 \phi_{i+1}^{n}+\phi_{i+2}^{n}\right)
\end{aligned}
$$

In the forms of $\phi_{i-1 / 2}^{n}$ and $\phi_{i+1 / 2}^{n}$, it is clear that, in absence of the second term on the right hand side, the rest of the equations are quite linear interpolation approximations to the constituent values. Also, the values inside the parentheses of the second term divided by $\Delta x^{2}$ represent the curvature of the constituent at the middle value. In other word, the QUICK scheme is basically a central differencing corrected by an upstream curvature.

For more simplification, the relevant positive flow forms of $\phi_{i-1 / 2}^{n}$ and $\phi_{i+1 / 2}^{n}$ can be written as follows:

$$
\begin{aligned}
& \phi_{i-1 / 2}^{n}=\frac{6}{8} \phi_{i-1}^{n}+\frac{3}{8} \phi_{i}^{n}-\frac{1}{8} \phi_{i-2}^{n} \\
& \phi_{i+1 / 2}^{n}=\frac{6}{8} \phi_{i}^{n}+\frac{3}{8} \phi_{i+1}^{n}-\frac{1}{8} \phi_{i-1}^{n}
\end{aligned}
$$

, and for the negative flow forms:

$$
\begin{aligned}
& \phi_{i-1 / 2}^{n}=\frac{6}{8} \phi_{i}^{n}+\frac{3}{8} \phi_{i-1}^{n}-\frac{1}{8} \phi_{i+1}^{n} \\
& \phi_{i+1 / 2}^{n}=\frac{6}{8} \phi_{i+1}^{n}+\frac{3}{8} \phi_{i}^{n}-\frac{1}{8} \phi_{i+2}^{n}
\end{aligned}
$$

Similar forms can be written for the advection in the $y$ and $z$-direction. Then, $\phi_{i-1 / 2}^{n}$ and $\phi_{i+1 / 2}^{n}$ are used to calculate the advective terms, $\left.a d v\right|_{i, j, k} ^{n}$. 


\section{The QUICKEST scheme:}

The QUICKEST scheme estimates the values at the interfaces by implementing the Quadratic Upstream Interpolation too, but it also approximates those values by taking the average over a time step $\Delta t$ rather than using the constituent value at a time level, $n$. Series of derivations (Leonard, 1979) starts by integrating the purely advective process over a time increment and then using the interfaces values of the QUICK scheme to estimate the constituent values at the old and new time level. This leads to the following approximations to the constituent values at the interfaces for the $x$-direction advective, positive flow, and uniform grid spacing:

$$
\begin{gathered}
\phi_{i-1 / 2}^{n}=\frac{1}{2}\left(\phi_{i-1}^{n}+\phi_{i}^{n}\right)-\frac{\Delta x}{2} \frac{u_{i-1}^{n} \Delta t}{\Delta x} G R A D_{i-1 / 2}-\frac{\Delta x^{2}}{6}\left(1-\left[\frac{u_{i-1}^{n} \Delta t}{\Delta x}\right]^{2}\right) C U R V_{i-1 / 2} \\
\phi_{i+1 / 2}^{n}=\frac{1}{2}\left(\phi_{i}^{n}+\phi_{i+1}^{n}\right)-\frac{\Delta x}{2} \frac{u_{i}^{n} \Delta t}{\Delta x} G R A D_{i+1 / 2}-\frac{\Delta x^{2}}{6}\left(1-\left[\frac{u_{i}^{n} \Delta t}{\Delta x}\right]^{2}\right) C U R V_{i+1 / 2}
\end{gathered}
$$

Where:

$$
\begin{array}{ll}
\operatorname{GRAD}_{i-1 / 2}=\frac{1}{\Delta x}\left(\phi_{i}^{n}-\phi_{i-1}^{n}\right) & \operatorname{CURV}_{i-1 / 2}=\frac{1}{\Delta x^{2}}\left(\phi_{i-2}^{n}-2 \phi_{i-1}^{n}+\phi_{i}^{n}\right) \\
\operatorname{GRAD}_{i+1 / 2}=\frac{1}{\Delta x}\left(\phi_{i+1}^{n}-\phi_{i}^{n}\right) & \operatorname{CURV}_{i+1 / 2}=\frac{1}{\Delta x^{2}}\left(\phi_{i-1}^{n}-2 \phi_{i}^{n}+\phi_{i+1}^{n}\right)
\end{array}
$$

Similar expressions are obtained for the negative flow. The GRAD and $C U R V$ are the only difference between positive and negative flow:

$$
\begin{array}{ll}
\operatorname{GRAD}_{i-1 / 2}=\frac{1}{\Delta x}\left(\phi_{i-1}^{n}-\phi_{i}^{n}\right) & \operatorname{CURV}_{i-1 / 2}=\frac{1}{\Delta x^{2}}\left(\phi_{i-1}^{n}-2 \phi_{i}^{n}+\phi_{i+1}^{n}\right) \\
\operatorname{GRAD}_{i+1 / 2}=\frac{1}{\Delta x}\left(\phi_{i}^{n}-\phi_{i+1}^{n}\right) & \operatorname{CURV}_{i+1 / 2}=\frac{1}{\Delta x^{2}}\left(\phi_{i}^{n}-2 \phi_{i+1}^{n}+\phi_{i+2}^{n}\right)
\end{array}
$$


Similar forms can be written for the advection in the y and z-direction. Then, $\phi_{i-1 / 2}^{n}$ and $\phi_{i+1 / 2}^{n}$ are used to calculate the advective terms, $\left.a d v\right|_{i, j, k} ^{n}$.

\section{The ULTIMATE QUICKEST scheme:}

Details of implementation of the ULTIMATE QUICKEST scheme (Leonard, 1991) are shown in the CE-QUAL-W2 User Manual (Cole \& Wells, 2017).

\subsubsection{Numerical Solution of the Linear Algebraic Equations}

Each numerical solution of the free surface equation, momentums, and transport equation results in with a system of linear algebraic equations. This system can be arranged in a matrix form:

$$
[A][X]=[B]
$$

Where $[A]$ is the coefficients matrix which is a diagonally predominant, $[X]$ is the unknown column matrix, and $[B]$ is the right hand side coefficients column matrix.

There are several methods for solving a set of linear algebraic equations; some are more suitable than the others. Therefore, any suitable method can be used here. The form of the system of linear algebraic equations plays a major role in choosing the solution method. The solution of the momentums and transport equation ends with a coefficients matrix of tri-diagonal in which the main diagonal elements are positive and the two off-diagonal elements are negative. The most convenient direct method that is widely and easily used in soling systems of equations in which $[A]$ is a tri-diagonal matrix is Thomas algorithm, also called tri-diagonal matrix algorithm. This is the reason why we employed time splitting technique in solving the equations of momentums and transport; because it ends 
with a simple tri-diagonal matrix which is diagonally predominant and can be easily solved by Thomas algorithm.

For transport equation, the numerical solution ends with

$$
U \phi_{i, j, k-1}^{n+1}+C \phi_{i, j, k}^{n+1}+D \phi_{i, j, k+1}^{n+1}=R
$$

Where $U, C, D$, and $R$ are knows and $\phi$ is unknown.

This equation is written at each of the unknown nodal points along the water column from top to bottom to form a system of simultaneous linear algebraic equations for each water column. The general matrix form of this system can be written as follows:

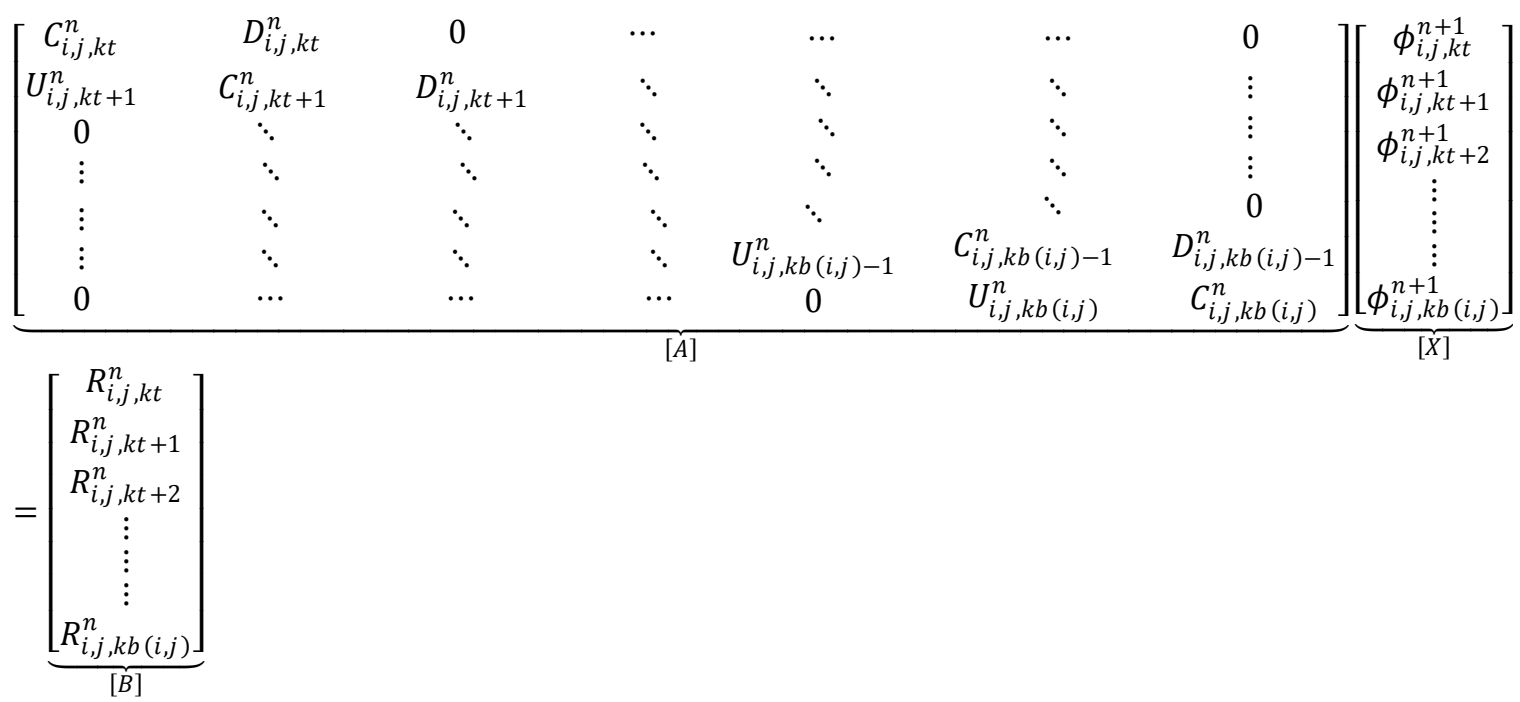

Thomas algorithm for solving this system of simultaneous linear algebraic equations is as follows:

- Calculate $\gamma_{i, j, k t}$ and $\alpha_{i, j, k t}$ : 


$$
\begin{aligned}
& \gamma_{i, j, k t}=\frac{D}{C} \\
& \alpha_{i, j, k t}=\frac{R}{C}
\end{aligned}
$$

- Calculate $\gamma_{i, j, k}$ and $\alpha_{i, j, k}$ for $k=k t+1, k t+2, \ldots \ldots \ldots, k b(i, j)$ :

$$
\begin{aligned}
\gamma_{i, j, k} & =\frac{D}{C-\left(U * \gamma_{i, j, k-1}\right)} \\
\alpha_{i, j, k} & =\frac{R-\left(U * \alpha_{i, j, k-1}\right)}{C-\left(U * \gamma_{i, j, k-1}\right)}
\end{aligned}
$$

- Set $\phi_{i, j, k b(i, j)}^{n+1}=\alpha_{i, j, k b(i, j)}$

- Calculate $\phi_{i, j, k}^{n+1}$ for $k=k b(i, j)-1, k b(i, j)-2, \ldots \ldots \ldots, k t$ :

$$
\phi_{i, j, k}^{n+1}=\alpha_{i, j, k}-\left(\gamma_{i, j, k} * \phi_{i, j, k+1}^{n+1}\right)
$$

A similar algorithm is used for the momentum equation solution.

While the solution of the momentum and transport equations ends with a coefficient matrix of tri-diagonal, the free surface equation solution ends with a coefficient matrix that is penta-diagonal for a rectangular domain and a sparse matrix otherwise. Using direct methods for solving the simultaneous linear algebraic equations are much more complicated and require rather large amount of computer storage and time; therefore, iterative methods could be used such as the conjugate gradient method. However, direct methods are more accurate than iterative methods. In the present model, the line-by-line method, a combination of Thomas algorithm method and Gauss-Sidel method, has been used (Patankar, 1980) to solve the system of simultaneous linear algebraic equations generated from the numerical solution of free surface equation. 
The system that results from the semi-implicit solution of the free surface equation is

$$
W \eta_{i-1, j}^{n+1}+N \eta_{i, j-1}^{n+1}+C \eta_{i, j}^{n+1}+S \eta_{i, j+1}^{n+1}+E \eta_{i+1, j}^{n+1}=R
$$

This equation is written at each of the unknown water surface elevations (sweeping in either direction and ordering line by line) to form a system of simultaneous linear algebraic equations. If we wrote this system in a matrix form for a rectangular domain, the matrix $[A]$ is a penta-diagonal coefficients matrix which is similar to the tri-diagonal matrix but it has additional two diagonals, above and below the band of the three diagonals by an equal offset.

In the line by line method, one direction of the system is assumed unknown, and the other direction is considered known to be taken from the latest values. Therefore, the system of the penta-diagonal coefficients matrix is solved as a system of tri-diagonal coefficients matrix, which needs a low cost algorithm to implement. Figure 2-9 and Figure 2-10 show the computational time cost of the sparse matrix direct solver (Press et al., 1992) and the line-by-line method solver in addition to the associated water balance \% error running both solvers based on the same input data of test 4 for the rectangular physical domain.

Thus, the penta-diagonal coefficient matrix for a rectangular domain was transferred to a tri-diagonal coefficient matrix, which can easily be solved by the Thomas algorithm. In the present work, we solved the tri-diagonal system as follows:

$$
W \eta_{i-1, j}^{n+1}+C \eta_{i, j}^{n+1}+E \eta_{i+1, j}^{n+1}=R-N \eta_{i, j-1}^{n}-S \eta_{i, j+1}^{n}
$$


The model sweeps in the $x$-direction (main stream direction), line-by-line, to transfer flow along the main flow direction. Also, another advantage of using line-by-line method other than the low computational cost is that the method helps to deal with irregular boundaries in which each row of neighboring unknowns is solved separately by Thomas algorithm.

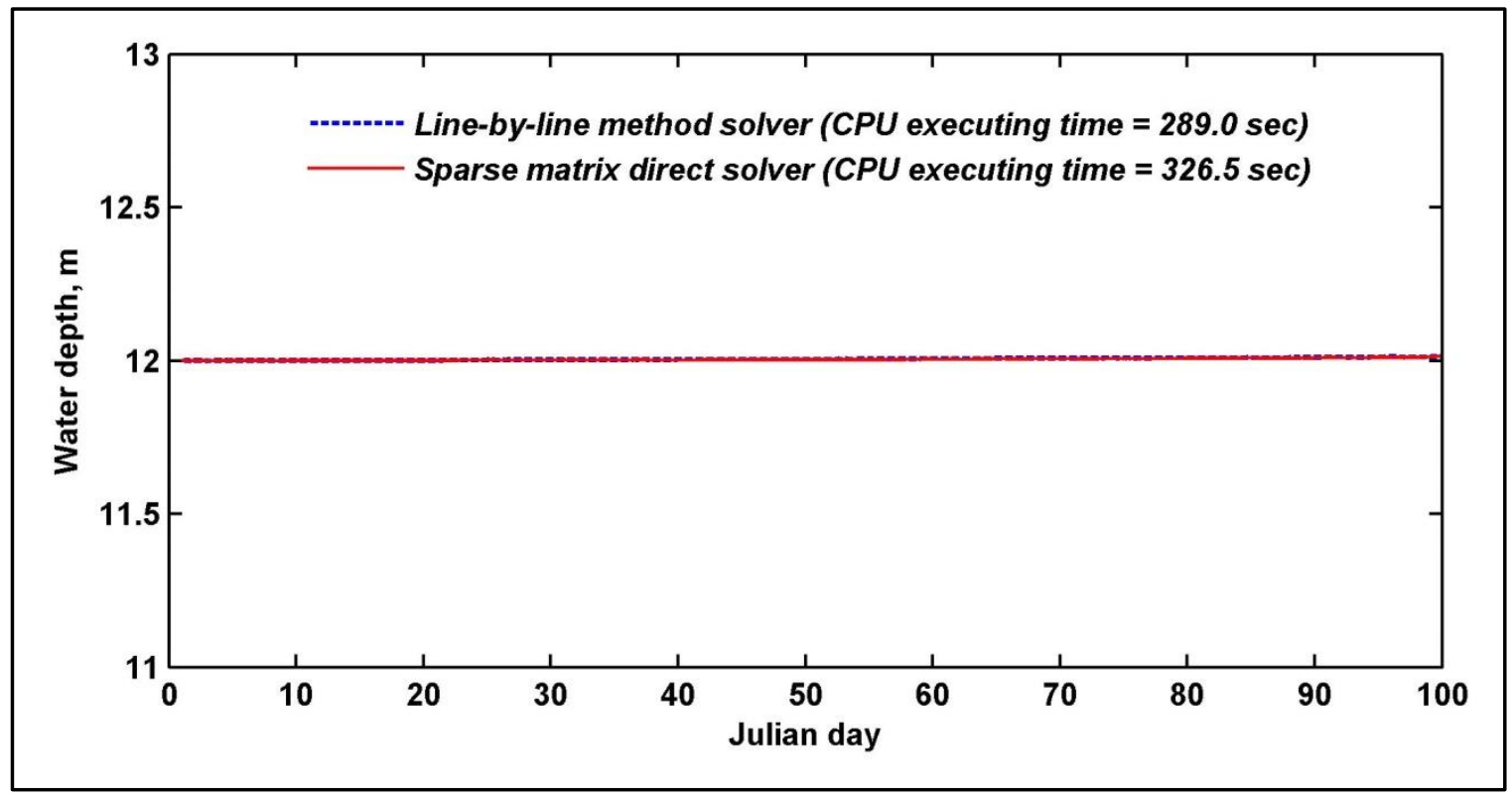

Figure 2-9. Comparison in the water depth between the sparse matrix direct solver and the line-by-line method solver 


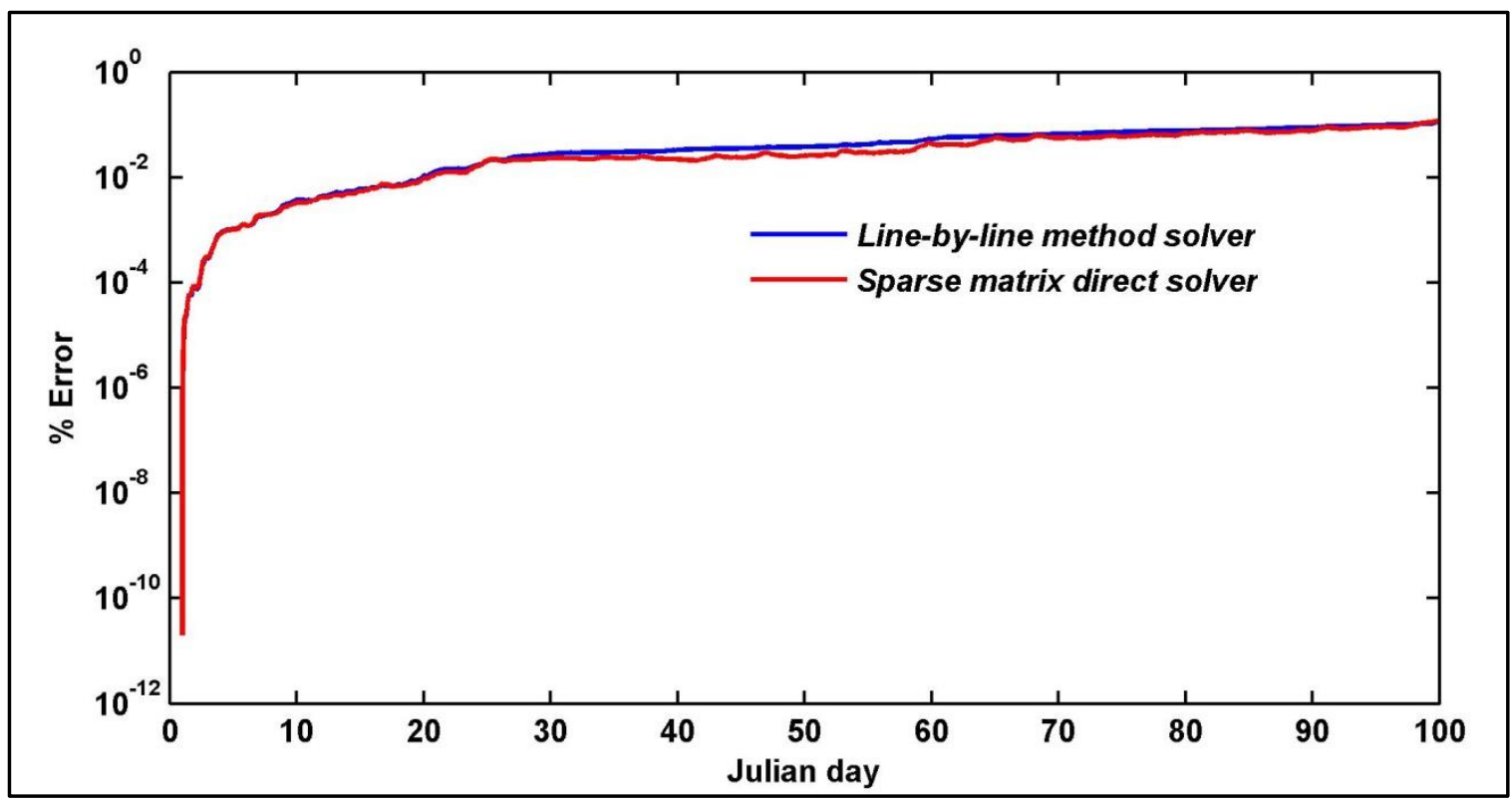

Figure 2-10. Comparison in the water balance generated by the sparse matrix direct solver and the line-by-line method solver

\subsubsection{Stability Criteria}

The model numerical stability is checked every single time step to make sure that the model is stable during the simulation time. It has been found that the present three-

dimensional model is always stable if the following stability condition is satisfied:

$$
\Delta t \leq\left[\left|\frac{\bar{u}_{i, j, k}}{\Delta x}\right|+\left|\frac{\bar{v}_{i, j, k}}{\Delta y}\right|+\left|\frac{\bar{w}_{i, j, k}}{\Delta z}\right|+2 \frac{\max \left(v_{h}, D x\right)}{\Delta x^{2}}+2 \frac{\max \left(v_{h}, D y\right)}{\Delta y^{2}}\right]^{-1}
$$

This stability condition is the same stability condition that was given by Vincenzo Casulli and Cheng (1992) even though the degree of implicitness has been added in the present numerical scheme. 
The present work is also giving the user an option to check the stability related to the celerity even though this option leads to less time step.

$$
\max \left(\frac{\sqrt{g D_{i, j}} \Delta t}{\Delta x}, \frac{\sqrt{g D_{i, j}} \Delta t}{\Delta y}\right) \leq 1
$$

Where $D_{i, j}$ is the total depth at the water column $(i, j)$.

\subsubsection{Model Procedure and Programming}

The procedure of the present numerical model involves the solution of the governing equations together with the auxiliary equations numerically under sufficient initial and boundary conditions to describe the considered problem. A computer program was written in Fortran 90 using Intel Visual Fortran Compiler to include all the above numerical solutions. The flow chart of the code is as shown in Figure 2-11. 


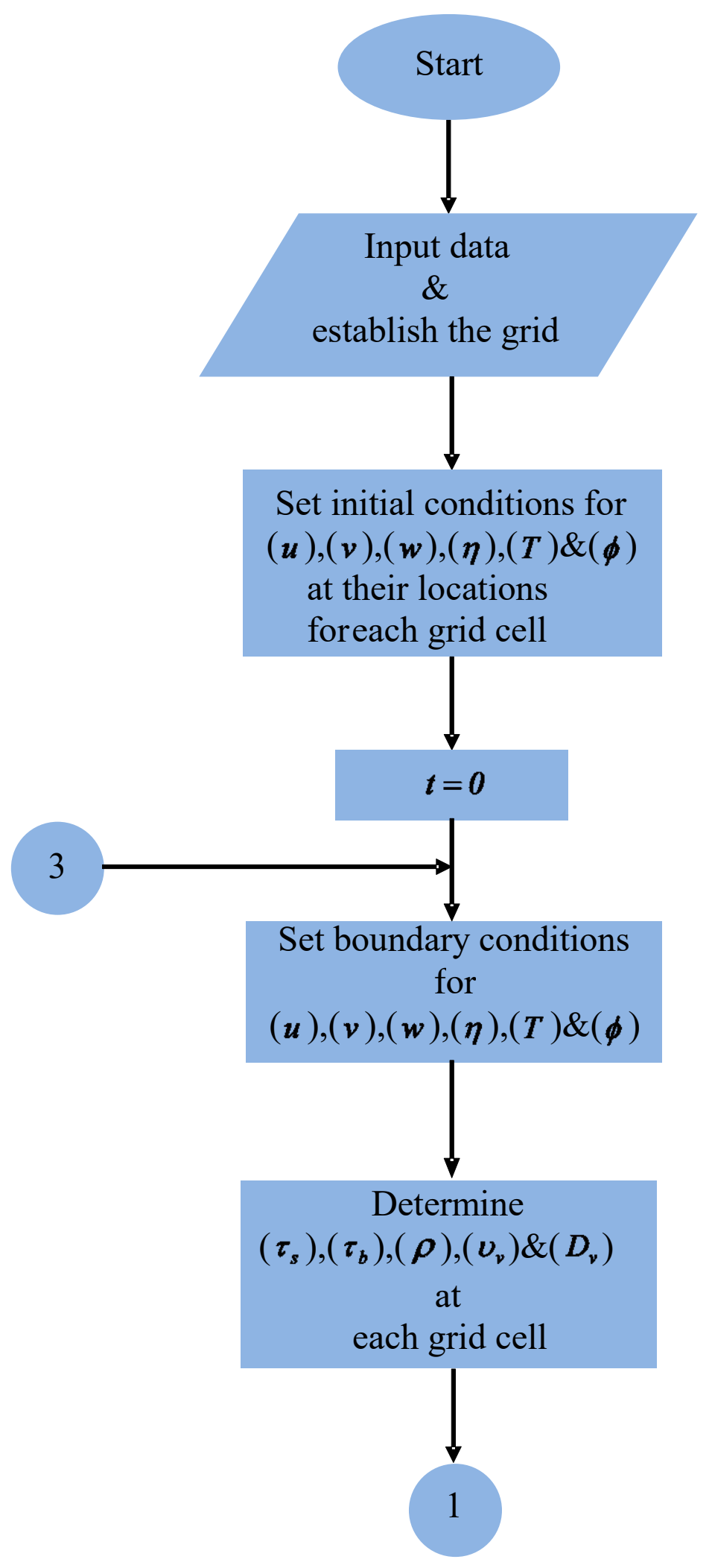




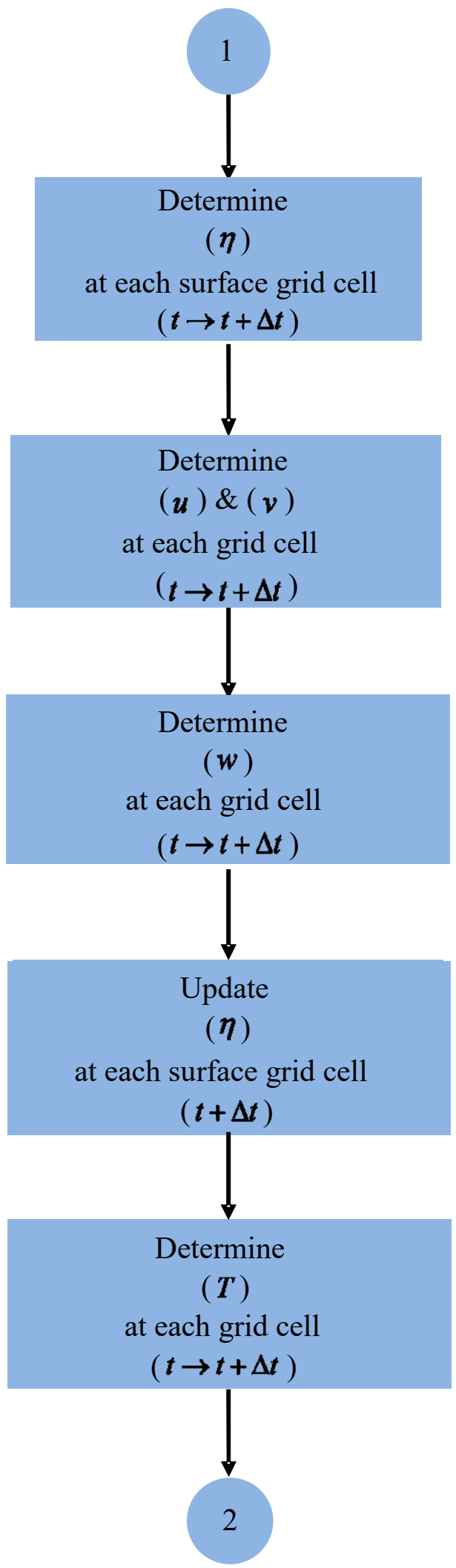




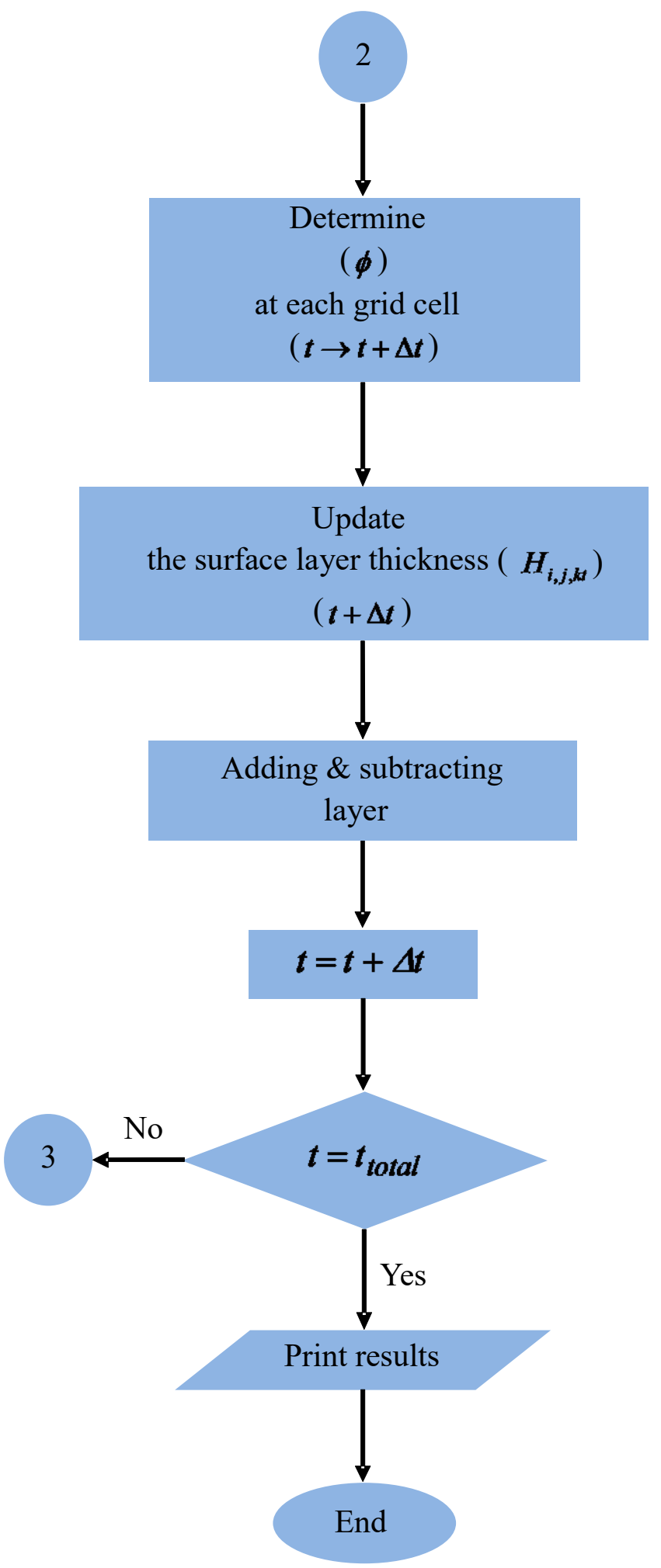

Figure 2-11. Flow chart of the code 


\subsection{Heat Sources and Sinks}

The 3D model is calculating the source/sink term of the heat transport equation by using the following equation in which $\phi$ is the temperature $(T)$ in ${ }^{\circ} \mathbf{C}$ :

$$
S_{T}=\frac{H_{n}}{\rho c_{p} H}
$$

Where:

$S_{T}=$ the heat source/sink term $\left({ }^{\circ} \mathrm{C} / \mathrm{sec}\right)$,

$H_{n}=$ the net surface heat flux into the water surface $\left(\mathrm{Wat} / \mathrm{m}^{2}\right)$,

$\rho c_{p}=$ a constant $\left(4182000 \mathrm{Joule} / \mathrm{m}^{3} /{ }^{\circ} \mathrm{C}\right)$, and

$H=$ the layer depth of the computational grid cell (m).

Surface heat exchange through the water-atmospheric interface is a significant factor controlling the amount of energy in and out of a waterbody. The amount of energy entering or leavings the waterbody can be represented in term of fluxes $\left(\mathrm{Watt} / \mathrm{m}^{2}\right)$, in which the flux in is positive (+) and the flux out is negative (-). The fluxes are then added to the normalized source/sink term of the heat transport governing equation. Surface heat fluxes calculation techniques have been reported in many references (Ahsan \& Blumberg, 1999; Chapra, 1997; Cole \& Buchak, 1995; Cole \& Wells, 2017; Edinger, Brady, \& Geyer, 1974; Ryan \& Harleman, 1973; Thomann \& Mueller, 1987; Vreugdenhil, 1989; Wunderlich, 1972).

In the present 3D model, the surface heat fluxes were formulated based on the term byterm process described by Cole and Wells (2017) and suggested by Edinger et al. (1974). Surface heat fluxes consist of five components as shown in Figure 2-12. Some of these 
components depend on water temperature (evaporation, conduction, and back-radiation), others (short and long radiations) depend on external conditions such as air temperature, cloud cover, and air moisture content. Evaporation and conduction are also affected by wind speed and direction. Solar short wave radiation and atmospheric long wave radiation are not a function of water temperature.

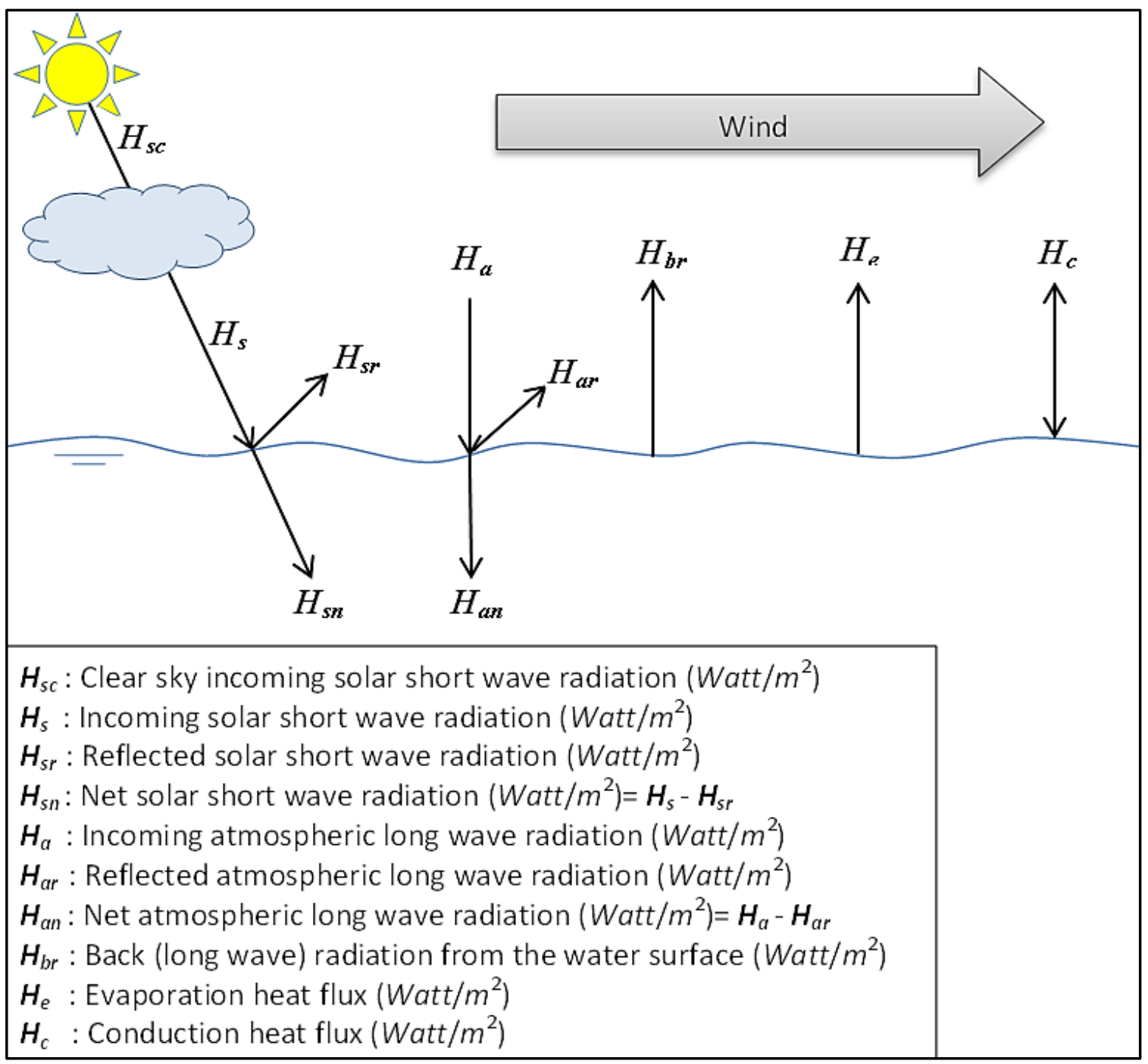

Figure 2-12. Components of the surface heat fluxes 
Thus, the net surface heat flux into the water surface $\left(\mathrm{Watt} / \mathrm{m}^{2}\right)$ is

$$
H_{n}=H_{s n}+H_{a n}-H_{b r}-H_{e}-H_{c}
$$

Typical magnitudes of the surface heat flux components in Watt $/ \mathrm{m}^{2}$ are as shown below (Shanahan, 1985):

Solar radiation, 50 to 350

Atmospheric radiation, 200 to 400

Back radiation, 250 to 500

Evaporation, 0 to 35

Conduction, -70 to 200

The model computes each term separately in Watt $/ \mathrm{m}^{2}$ as shown below.

\subsubsection{Solar Short Wave Radiation}

The net solar short wave radiation $\left(H_{s n}\right)$ is calculated from the following equation (Wunderlich, 1972):

$$
H_{s n}=H_{s c}\left(1-0.65 C^{2}\right)
$$

Where $C$ is the sky fraction covered by clouds $(0-1)$, and the term $\left(1-0.65 C^{2}\right)$ accounts for the cloud cover.

$H_{s c}$ is either measured or calculated. Many models estimate the clear sky solar radiation theoretically based on sun position related to the site and cloudiness in different ways. Annear and Wells (2007) reported five models to estimate clear sky solar radiation and performed a comparison between them and field data. 
EPA (1971) developed a model to estimate clear sky solar radiation in Btu/ft ${ }^{2} /$ day. Cole and Wells (2017) has implemented the EPA (1971) model after refining the equations for CE-QUAL-W2.

The 3D model can either compute clear sky solar radiation theoretically and adjust it by cloud cover or read in measured short-wave solar radiation directly from the available meteorological data.

\subsubsection{Atmospheric Long Wave Radiation}

The net atmospheric long wave radiation $\left(H_{a n}\right)$ is computed using an approach proposed by Wells et al. (1982):

For air temperature $\left(T_{a}\right) \geq 5^{\circ} \mathrm{C}$, the atmospheric clear sky long wave radiation is calculated using the approach of Swinbank (1963):

$$
H_{a}=\sigma \alpha_{\circ}\left(T_{a}+273\right)^{6}
$$

For air temperature $\left(T_{a}\right)<5{ }^{\circ} \mathrm{C}$, the atmospheric clear sky long wave radiation is calculated using the approach of Idso and Jackson (1969):

$$
H_{a}=\sigma\left(T_{a}+273\right)^{4}\left[1-0.261 e^{\left(-7.77 E-4 T_{a}^{2}\right)}\right]
$$

Where $\sigma$ is the Stefan-Boltzmann constant $\left(5.62 \mathrm{E}-8 \mathrm{Watt} / \mathrm{m}^{2} /{ }^{\circ} \mathrm{K}^{4}\right), \alpha_{\circ}$ is a proportionality constant $(0.937 \mathrm{E}-5)$, and $T_{a}$ is the air temperature $\left({ }^{\circ} \mathrm{C}\right)$.

Adding the reflectivity and the cloudiness effect, the final equations for calculating the net the atmospheric long wave radiation can be written as follows: 
For air temperature $\left(T_{a}\right) \geq 5^{\circ} \mathrm{C}$,

$$
H_{a n}=\epsilon \sigma \alpha_{\circ}\left(T_{a}+273\right)^{6}\left(1+0.17 C^{2}\right)
$$

For air temperature $\left(T_{a}\right)<5^{\circ} \mathrm{C}$,

$$
H_{a n}=\epsilon \sigma\left(T_{a}+273\right)^{4}\left[1-0.261 e^{\left(-7.77 E-4 T_{a}^{2}\right)}\right]\left(1+0.17 C^{2}\right)
$$

Where $\epsilon$ is the emissivity of the waterbody (0.97), and $\left(1+0.17 C^{2}\right)$ accounts for the cloud cover effect (Wunderlich, 1972).

\subsubsection{Back (Long Wave) Radiation from the Water Surface}

The back radiation $\left(H_{b r}\right)$ is calculated as follows based on the water surface temperature $\left(T_{s}\right)$ :

$$
H_{b r}=\epsilon \sigma\left(T_{s}+273\right)^{4}
$$

Where $\epsilon$ is the emissivity of the waterbody (0.97), $\sigma$ is the Stefan-Boltzmann constant (5.62E-8 Watt $\left./ \mathrm{m}^{2} /{ }^{\circ} \mathrm{K}^{4}\right)$, and $T_{S}$ is the water surface temperature $\left({ }^{\circ} \mathrm{C}\right)$.

\subsubsection{Evaporation Heat Flux}

The evaporation heat flux $\left(H_{e}\right)$ is computed using the approach proposed by Cole and Wells (2017):

$$
H_{e}=f(W)\left(e_{s}-e_{a}\right)
$$

Where:

$f(W)=$ the wind speed function (Watt $\left./ \mathrm{m}^{2} / \mathrm{mmHg}\right)$, $W=$ the wind speed measured at $2 m$ above the water surface $(\mathrm{m} / \mathrm{sec})$. 
$e_{s}=$ the saturated vapor pressure at $T_{s}(\mathrm{mmHg})$, and

$e_{a}=$ the atmospheric vapor pressure at $2 m$ above the water surface $(\mathrm{mmHg})$.

Many different expressions have been developed for the wind speed function (Helfrich et al., 1982; Shanahan, 1985). Most of these expressions can be written in a general form:

$$
f(W)=a+b W^{c}
$$

Where $a, b$, and $c$ are empirical coefficients. The following values were suggested by Edinger et al. (1974) $: a=9.4 \mathrm{Watt} / \mathrm{m}^{2} / \mathrm{mmHg}, b=0.46 \mathrm{Watt} / \mathrm{m}^{2} / \mathrm{mmHg} /(\mathrm{m} / \mathrm{sec})^{2}$., and $c=2$.

Another evaporation formula that by Ryan and Harleman (1973),

$$
f(W)=a+b W
$$

Where:

$b=$ a constant $\left(4.26 \mathrm{Watt} / \mathrm{m}^{2} / \mathrm{mmHg} / \mathrm{m} / \mathrm{sec}\right)$, and $a=$ a parameter depends on the difference in the virtual temperature $\left(T_{v}\right)$ between at the water surface $\left(T_{s v}\right)$ and in the air at $2 \mathrm{~m}$ above the water surface $\left(T_{a v}\right)$,

$$
a=\lambda\left(T_{s v}-T_{a v}\right)^{1 / 3}
$$

Where $\lambda$ is a constant $\left(3.59 \mathrm{Watt} / \mathrm{m}^{2} / \mathrm{mmHg} /{ }^{\circ} \mathrm{C}^{1 / 3}\right)$.

The virtual temperature is the temperature of dry air if it has the density of the moist air. The virtual temperature reflects the buoyancy effect of the moist air above the heated water surface. 


$$
T_{v}=(T+273) /[1-0.378(e / p)]
$$

Where $e$ is the vapor pressure in $\mathrm{mmHg}\left(e_{s}\right.$, or $e_{a}$ corresponding to $T_{s v}$, or $T_{a v}$ respectively), and $p$ is the atmospheric pressure $(760 \mathrm{mmHg})$.

If $\left(T_{s v}-T_{a v}\right)$ was negative or less than that determined based on Lake Hefner, the quantity $\left(T_{s v}-T_{a v}\right)$ in the formulation of Ryan and Harleman (1973) becomes $\left(0.0084 W^{3}\right)$ and $f(W)$ returns to the Lake Hefner model $(a=0, b=4.99$ Watt $/ \mathrm{m}^{2} / \mathrm{mmHg} / \mathrm{m} / \mathrm{sec}$, and $c=1$ ).

The saturated vapor pressure is a function of water surface temperature and can be calculated based on relative humidity $\left(R_{h}\right)$ of unity (Chapra, 1997):

$$
e_{s}=4.596 E X P\left(\frac{17.27 T_{s}}{T_{S}+273.3}\right)
$$

Also, the air vapor pressure is based on ambient air temperature and air relative humidity.

$$
e_{a}=R_{h} e_{s}
$$

Air vapor pressure can be calculated as follows based on dew point temperature (Chapra, 1997):

$$
e_{a}=4.596 E X P\left(\frac{17.27 T_{d}}{T_{d}+273.3}\right)
$$

Where $T_{d}$ is the dew point temperature $\left({ }^{\circ} \mathrm{C}\right)$. 


\subsubsection{Conduction Heat Flux}

The conduction process is a result of heat diffusion which is similar to the mass diffusion that controls the evaporation process. Thus, conduction heat flux $\left(H_{c}\right)$ is proportion to the evaporation heat flux and the proportional constant is called Bowen's ratio. Cole and Wells (2017) computed the conducted heat flux as follows:

$$
H_{c}=C_{c} f(W)\left(T_{s}-T_{a}\right)
$$

Where $C_{c}$ is the Bowen's coefficient $\left(0.47 \mathrm{mmHg} /{ }^{\circ} \mathrm{C}\right)$.

\subsubsection{Wind Speed Measurements}

The heat budget calculations require wind to be measured at $2 \mathrm{~m}$ above the water surface. If the available wind data were measured at known height other than $2 \mathrm{~m}$, wind speed should be adjusted to $2 \mathrm{~m}$ height. Ryan and Harleman (1973) proposed that wind speed has a logarithmic profile distribution above the water surface by the following formula:

$$
\frac{W_{Z}}{W_{Z 1}}=\frac{\ln \left(\frac{Z}{Z_{\circ}}\right)}{\ln \left(\frac{Z_{1}}{Z_{\circ}}\right)}
$$

Where:

$W_{z}=$ the desired wind velocity $(\mathrm{m} / \mathrm{sec})$ at elevation $z(\mathrm{~m})$,

$W_{Z 1}=$ the known wind velocity $(\mathrm{m} / \mathrm{sec})$ at elevation $\mathrm{z}_{1} .(\mathrm{m})$, and

$Z_{\circ}=$ the wind roughness height $(\mathrm{m})$.

The wind roughness height was researched by Helfrich et al. (1982). High wind speed increases the action of surface water waves. Thus, the higher the wind speed, the higher 
the roughness height. Ryan and Harleman (1973) used a roughness height of $0.001 \mathrm{~m}$ for wind velocity $<2.2 \mathrm{~m} / \mathrm{sec}$ and $0.0049 \mathrm{~m}$ for wind velocity $\geq 2.2 \mathrm{~m} / \mathrm{sec}$. Cole and Wells (2017) used similar values. Also, because the roughness height above the water surface is less than that above land, transferring wind measured above land to another location above the water surface produces a large potential error. Thus, it is possible to increase wind speed for the large lakes to about factor of 2 (Helfrich et al., 1982).

\subsubsection{Required Meteorological Data}

Summing the five heat fluxes gives the net surface heat flux into the water surface in Watt $/ \mathrm{m}^{2}$. To calculate the heat budget, meteorological data must be prepared for the simulation period including air temperature, dew point, wind speed, wind direction, cloud cover, and solar radiation.

An example of the input csv file and the required units of the meteorological data is shown below: The headers of columns (left to right) are Julian days, air temperature, dew point, wind speed, wind direction, cloud cover, and solar radiation. The last column is blank indicating that the solar short radiation is going to be calculated by the model internally. 
Meteorological data input file (METFN.csv) example:

\begin{tabular}{|c|c|c|c|c|c|c|}
\hline \multicolumn{9}{|l|}{ \$ Lake Chaplain Met Data } & JDAY & TAIR, $^{\circ} \mathrm{C}$ & TDEW $^{\circ} \mathrm{C}$ & $\begin{array}{c}\text { WIND, } \\
\mathrm{m} / \mathrm{sec}\end{array}$ & $\begin{array}{c}\text { PHI, } \\
\text { radians }\end{array}$ & $\begin{array}{c}\text { CLOUD, } \\
(0-10)\end{array}$ & $\begin{array}{c}\text { SRO, } \\
\text { Watt } / \mathrm{m}^{2}\end{array}$ \\
\hline 222.347 & 12.7 & 11.42 & 1.34 & 2.93 & 10 & \\
\hline 222.358 & 12.8 & 11.35 & 0 & 2.86 & 10 & \\
\hline 222.368 & 12.8 & 11.18 & 0.45 & 2.32 & 10 & \\
\hline 222.378 & 13.1 & 11.31 & 0.89 & 2.15 & 10 & \\
\hline 222.389 & 13.2 & 11.06 & 0 & 1.94 & 10 & \\
\hline 222.396 & 13.6 & 11.28 & 0.45 & 2.41 & 10 & \\
\hline 222.403 & 13.6 & 11.1 & 0 & 2.25 & 10 & \\
\hline 222.413 & 13.9 & 10.85 & 0 & 3 & 10 & \\
\hline 222.42 & 14.3 & 10.87 & 0 & 3 & 10 & \\
\hline
\end{tabular}




\section{CHAPTER 3. MODEL VERIFICATION}

In the first step after developing the numerical model and before proceeding further to add more features or applying the model to real field case studies, it was necessary to test the foundation of the numerical scheme in order to determine its validity. Analytical verification of the model was done by comparing model predictions to known analytical exact solutions test cases. Furthermore, sensitivity tests were made exploring whether the code has balances volume, how the model responds to changes in bed resistance, and how wind influences the water flow dynamics.

\subsection{Test 1: Free Surface Seiching in a Closed Rectangular Basin}

This test was done in a similar way to that test recommended by Wang, Roache, Schmalz, Jia, and Smith (2009). A closed rectangular basin was subjected to an initial vertical displacement in which the free surface wave has a profile of a half cosine in the longitudinal direction as shown in Figure 3-1.

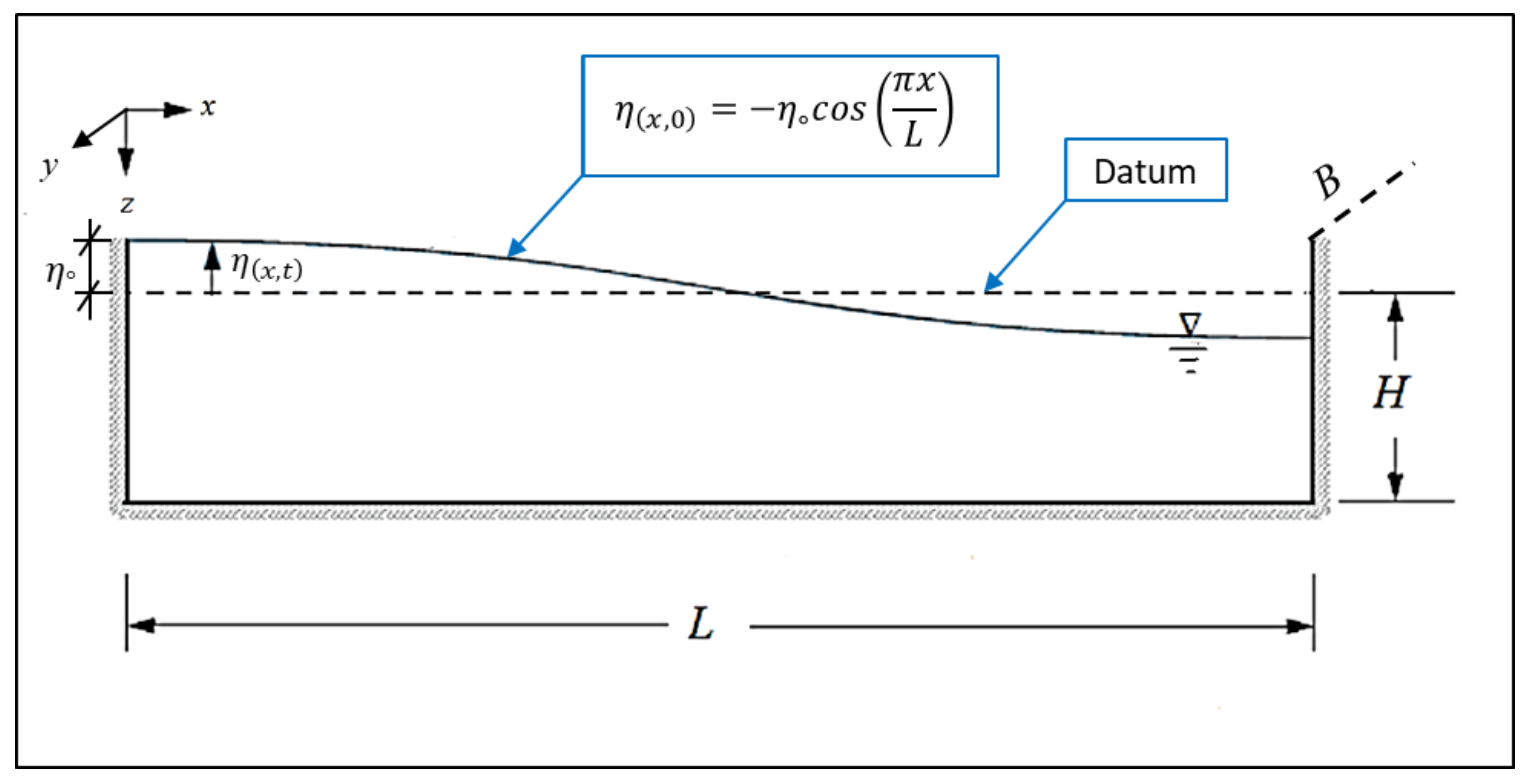

Figure 3-1. Seiching basin for the test 1 
The basin dimensions were:

$L=38000 \mathrm{~m}$,

$B=6000 \mathrm{~m}$, and

$H=12 \mathrm{~m}$.

The initial vertical amplitude at the left and right boundaries of the basin, $\eta_{0}$, was $25 \mathrm{~cm}$.

Thus, after releasing the system from the rest, the oscillated wave continues with time. If there were frictional resistance, the wave is damped and eventually the system goes to rest. Also, we make the following assumptions:

- At the closed boundaries, the longitudinal and lateral velocities are zero.

- The advection terms, diffusion terms, and boundary shear stresses are neglected.

- The fluid is inviscid and has a constant density, $\rho=1000 \mathrm{~kg} / \mathrm{m}^{3}$.

- $\quad$ The Coriolis force is neglected, $f=0$.

- There are no sources/sinks of fluid mass.

- Boussinesq approximations are valid and the pressure is hydrostatic.

Based on the above assumptions, the governing equations can be written as follows:

The free surface equation:

$$
\frac{\partial \eta}{\partial t}+\frac{\partial u H}{\partial x}=0
$$

The X-Momentum equation:

$$
\frac{\partial u}{\partial t}+g \frac{\partial \eta}{\partial x}=0
$$


The analytical solutions was given by Eliason and Bourgeois (1997) :

$\eta_{(x, t)}=\eta_{\circ} \cos \left(\frac{\pi x}{L}\right) \cos \left(\frac{\pi \sqrt{g H}}{L} t\right)$

$u_{(x, t)}=\frac{\eta_{\circ} \sqrt{g H}}{H} \sin \left(\frac{\pi x}{L}\right) \sin \left(\frac{\pi \sqrt{g H}}{L} t\right)$

Where $\sqrt{g H}$ is the gravity wave speed.

The one-dimensional governing equations and their analytical solutions are applicable for the case of upward positive $z$-direction. Thus, to match the present three-dimensional model setup, we need to modify the one-dimensional solution of $\eta_{(x, t)}$ by multiplying it by $(-1)$ to become:

$\eta_{(x, t)}=-\eta_{\circ} \cos \left(\frac{\pi x}{L}\right) \cos \left(\frac{\pi \sqrt{g H}}{L} t\right)$

Thus, based on the coordinate system setup of the present three-dimensional model, the one-dimensional governing equations that satisfy the test become:

The free surface equation:

$$
\frac{\partial \eta}{\partial t}-\frac{\partial u H}{\partial x}=0
$$

The X-Momentum equation:

$$
\frac{\partial u}{\partial t}-g \frac{\partial \eta}{\partial x}=0
$$

Subjected to Initial conditions: 


$$
\eta_{(x, 0)}=-0.25 \times \cos \left(\frac{\pi x}{L}\right) \quad u=v=w=0
$$

And boundary conditions of $(u=v=w=0)$ at the closed boundaries in addition to the following Neumann boundary conditions:

- Boundaries normal to the $x$-axis, $\frac{\partial v}{\partial x}=\frac{\partial \eta}{\partial x}=0$

- Boundaries normal to the $y$-axis, $\frac{\partial u}{\partial y}=\frac{\partial \eta}{\partial y}=0$

- Boundaries normal to the $z$-axis, $\frac{\partial u}{\partial z}=\frac{\partial v}{\partial z}=0$

The domain was divided into 1575 computational cells of $21 \times 5 \times 15(x, y, z)$ as shown in Figure 3-2, in which $k t=3$ and the total number of internal cells were 684 . The size of the computational cells was $\Delta x=\Delta y=2000 \mathrm{~m}$ and $\Delta z=1 \mathrm{~m}$.

The advection and diffusion terms, top and bottom shear stresses, and Coriolis force were set to zero in the model to agree with the analytical solution. To maintain a stable solution, the time step was satisfied by the gravity Courant number stability condition, $\sqrt{g H} \Delta t / \Delta x) \leq 1$. Thus, we used time steps of $\Delta t=100,70,50$, and 5 sec to explore the impact of time step on the model predictions compared to the analytical solution.

A comparison in the water level $(\eta)$ and longitudinal velocity $(u)$ between the model results and the analytical solution for the seiching basin by using the fully implicit scheme is shown in Figure 3-3 to Figure 3-10. The comparison was done near the left and right boundary of the basin. The distance between the selected left location for the comparison and the nearest boundary is equal to the distance between the selected right location and its nearest boundary, i.e. symmetric locations. This helps to ensure that if the 
solution were correct, the two waves at these locations would have the same magnitude at the same time of simulation but in opposite directions. For these runs, two time steps were chosen $(\Delta t=50$, and $5 \mathrm{sec})$ to examine how the time step affects wave damping. The results showed good agreement with the analytical solution even though there is damping of the numerical solution. The damping arises from using an implicit technique in the solution of the free surface equation (Vreugdenhil, 1989). The implicit scheme eliminates the celerity stability condition (Scott A. Wells, 2002a), however the solution still has wave damping.

Figure 3-11 and Figure 3-12 show the effect of time step on wave damping. Even though the criterion for the time step for stability was satisfied, the numerical code still had wave dampening for the higher time steps. But by reducing the time step below that required for stability, the damping decreased significantly. This agrees with Vreugdenhil (1989) who showed that "the time step is the major factor influencing the accuracy". The numerical solution will agree with the analytical solution without phase lag for any time step within the stability region. Wells (2002a) showed that running the model with a high time step that may be numerically stable does not guarantee numerical accuracy.

Furthermore, Figure 3-13 shows the model predictions of water level by using two spatial resolutions $(\Delta x=\Delta y=2000 \mathrm{~m}$ and $\Delta x=\Delta y=1000 \mathrm{~m})$ and same time step in which the model is stable for both resolutions. Both results of the model are almost the same, indicating that the model produces similar predictions with similar numerical behavior under similar time step. 
The damping rate of the free surface wave can be decreased by implementing the degree of implicitness ( $\theta$-method) of the semi-implicit scheme, in which a minimum damping rate can be achieved with $\theta=0.5$ (V. Casulli \& Cattani, 1994; Vreugdenhil, 1989). However, Vreugdenhil (1989) proposed using a value equal or close to 0.5 to take care instability resulting from nonlinear terms, usually 0.52 or 0.55 is recommended for the practical work. Figure 3-14 to Figure 3-17 show the model results using $\theta=0.55$ and 1 with $\Delta t=50 \mathrm{sec}$ and $5 \mathrm{sec}$ at a location close to the left boundary $(i=19, j=3$, and $k=k t=3)$. Therefore, the implementation of the semi-implicit scheme in the present 3D model improved the fully implicit scheme by reducing the wave damping of the numerical solution. The degree of implicitness $(\theta)$ can be chosen in the present 3D model depending on the user choice. However, using a value of 0.55 would be the best option based on numerical considerations relevant to the model. 


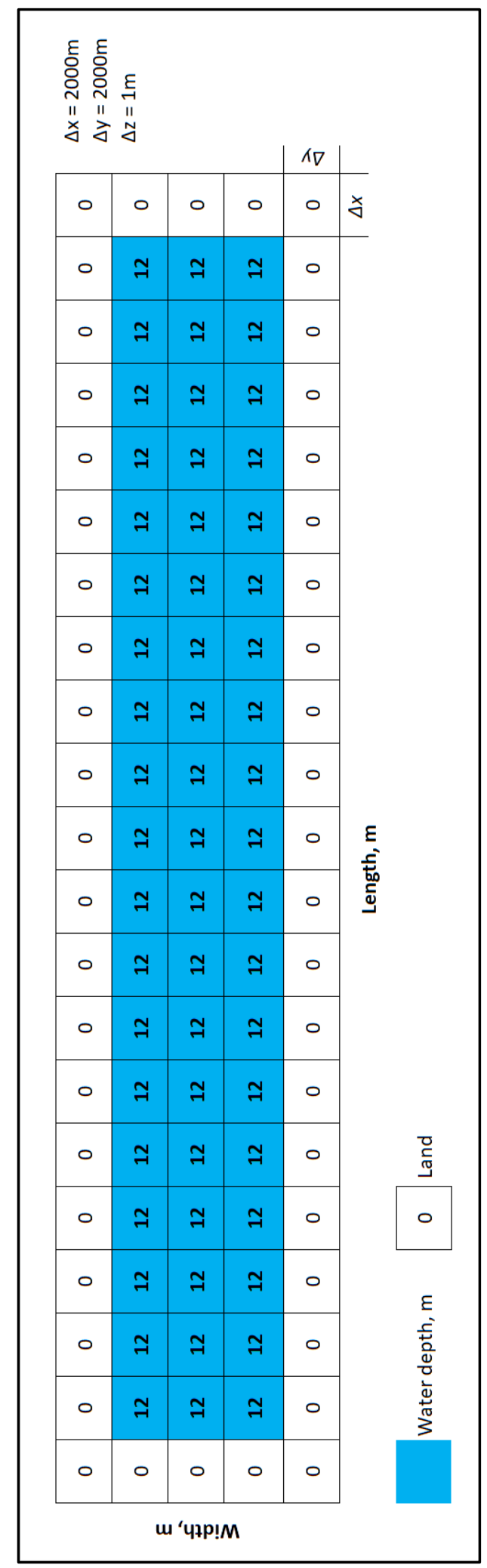

Figure 3-2. Test 1 basin domain and the input bathymetry 


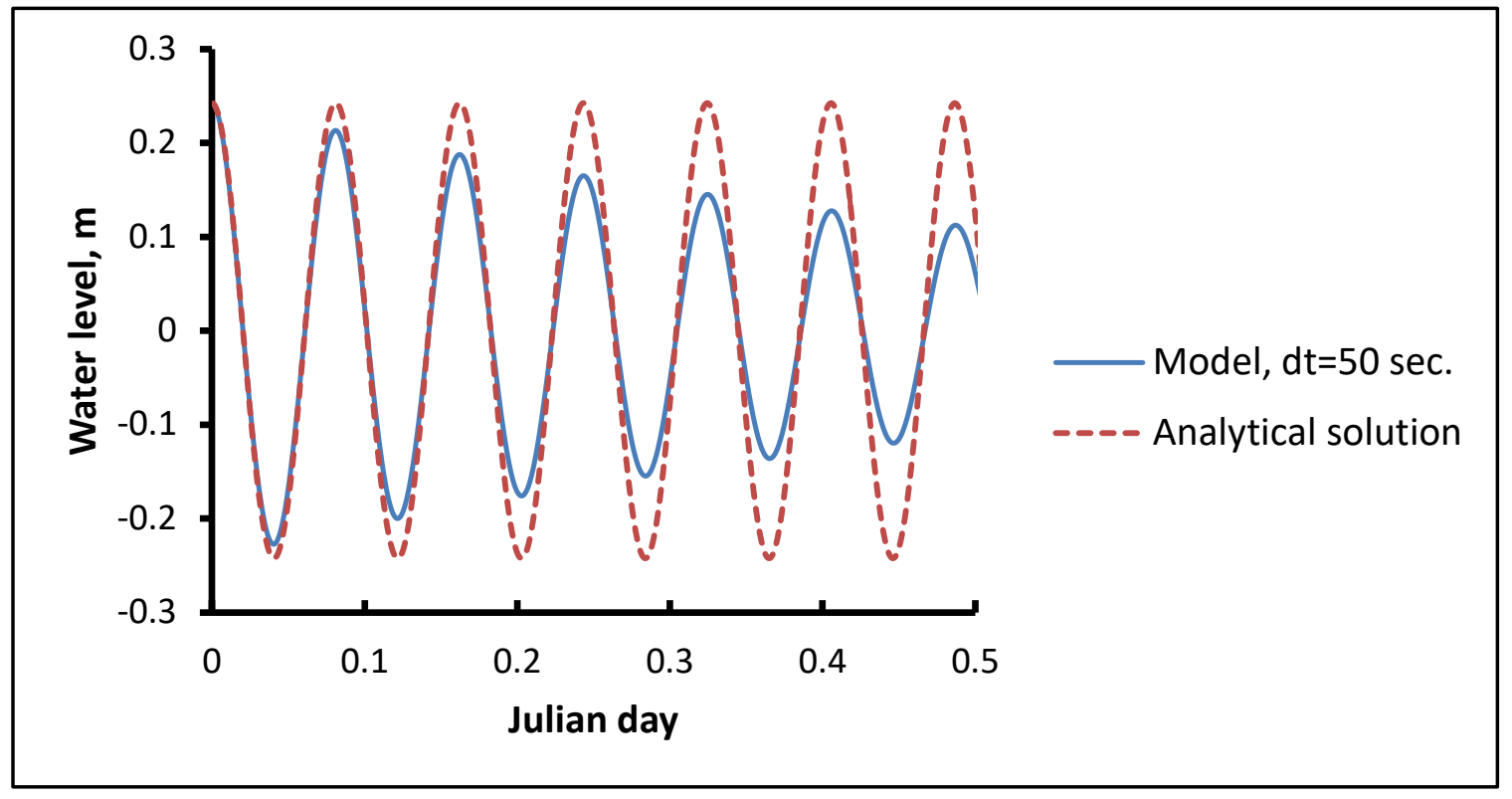

Figure 3-3. Comparison in the water level $(\eta)$ between the model results and the analytical solution for the seiching basin near the right boundary $(i=19, j=3$, and $k=k t=3), \Delta t=50$ sec

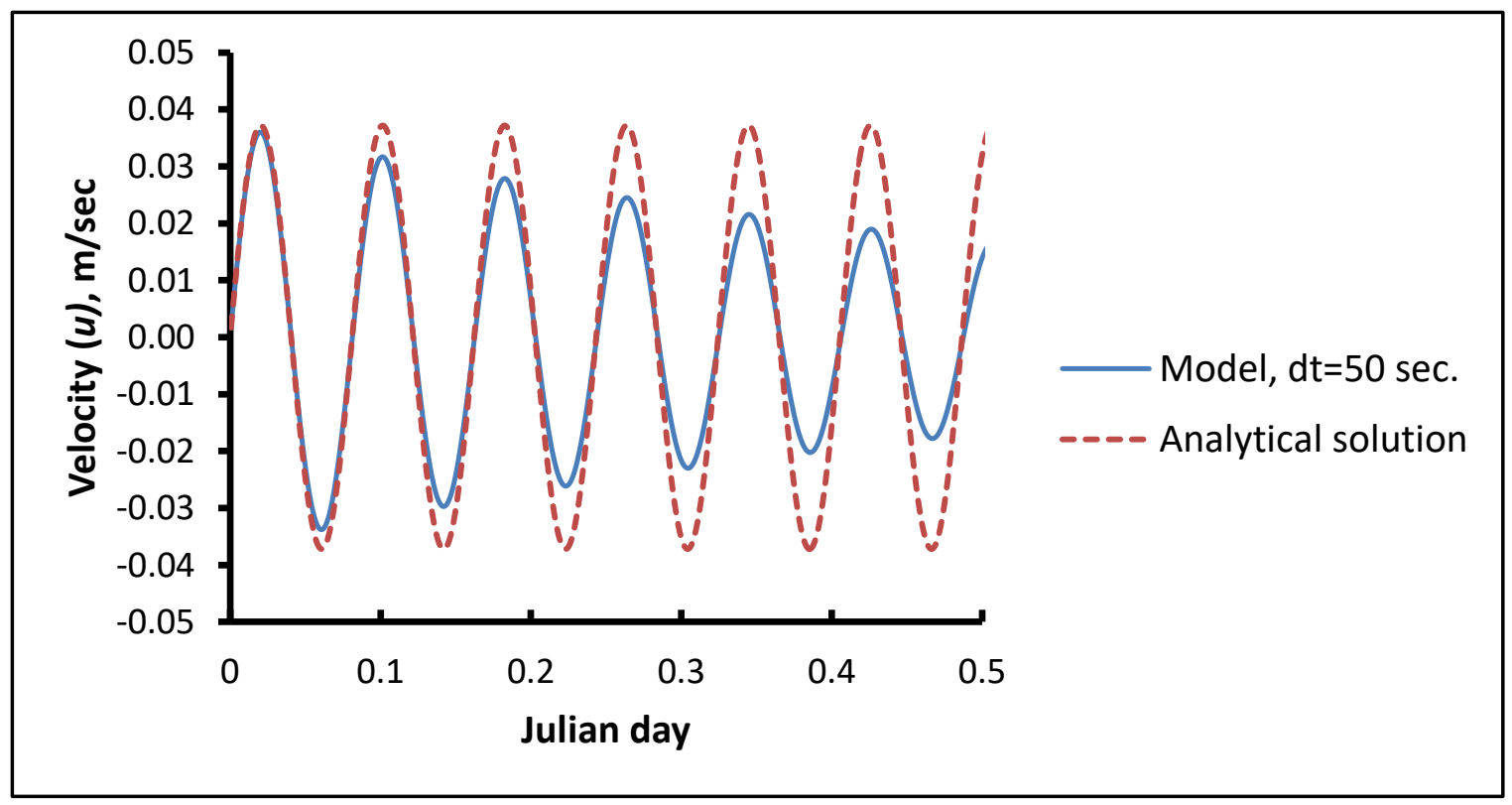

Figure 3-4. Comparison in the longitudinal velocity (u) between the model results and the analytical solution for the seiching basin near the right boundary $(i=19, j=3$, and $k=k t=3)$, $\Delta \mathrm{t}=\mathbf{5 0}$ sec 


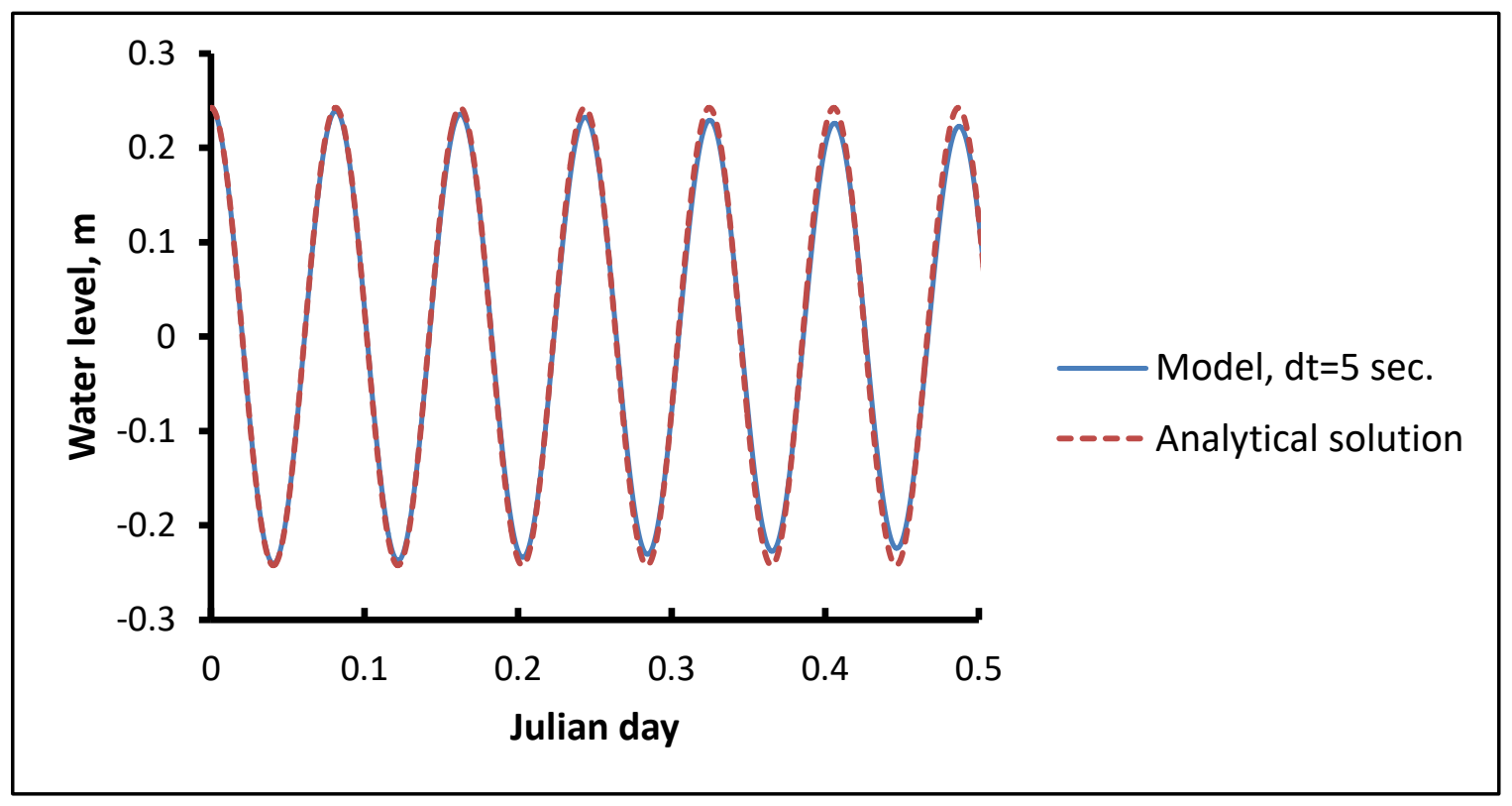

Figure 3-5. Comparison in the water level $(\eta)$ between the model results and the analytical solution for the seiching basin near the right boundary $(i=19, j=3$, and $k=k t=3), \Delta \mathrm{t}=5 \mathrm{sec}$

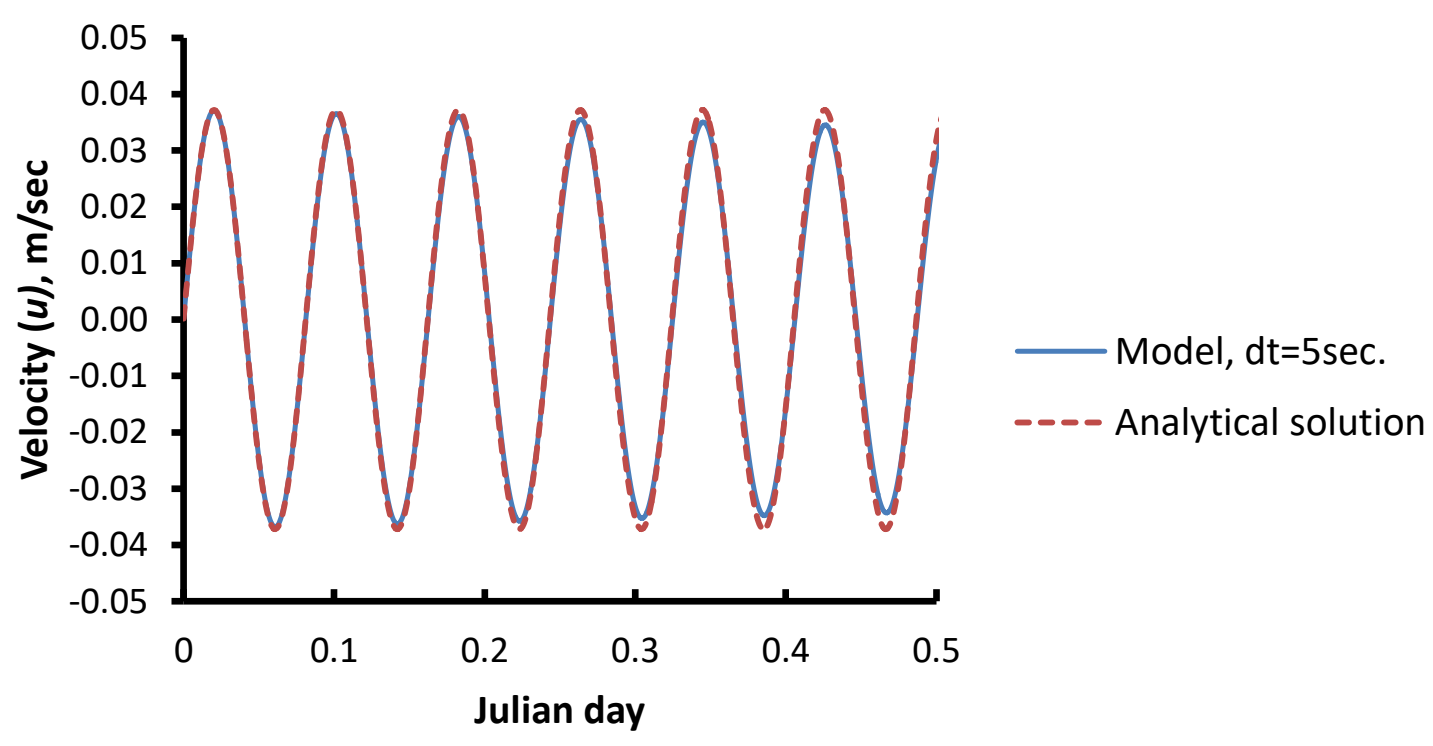

Figure 3-6. Comparison in the longitudinal velocity (u) between the model results and the analytical solution for the seiching basin near the right boundary $(i=19, j=3$, and $k=k t=3)$, $\Delta \mathrm{t}=\mathbf{5} \mathrm{sec}$ 


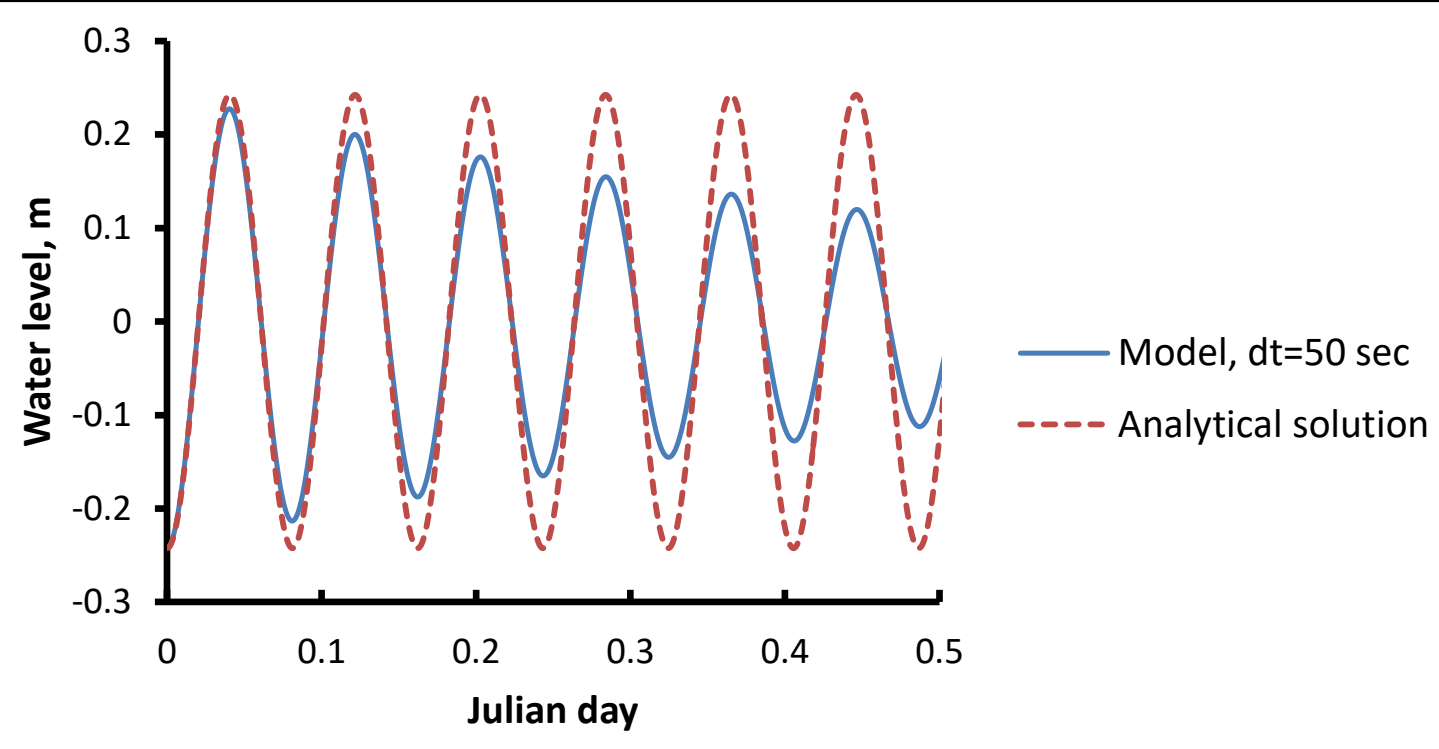

Figure 3-7. Comparison in the water level $(\eta)$ between the model results and the analytical solution for the seiching basin near the left boundary $(i=3, j=3$, and $k=k t=3), \Delta t=50$ sec

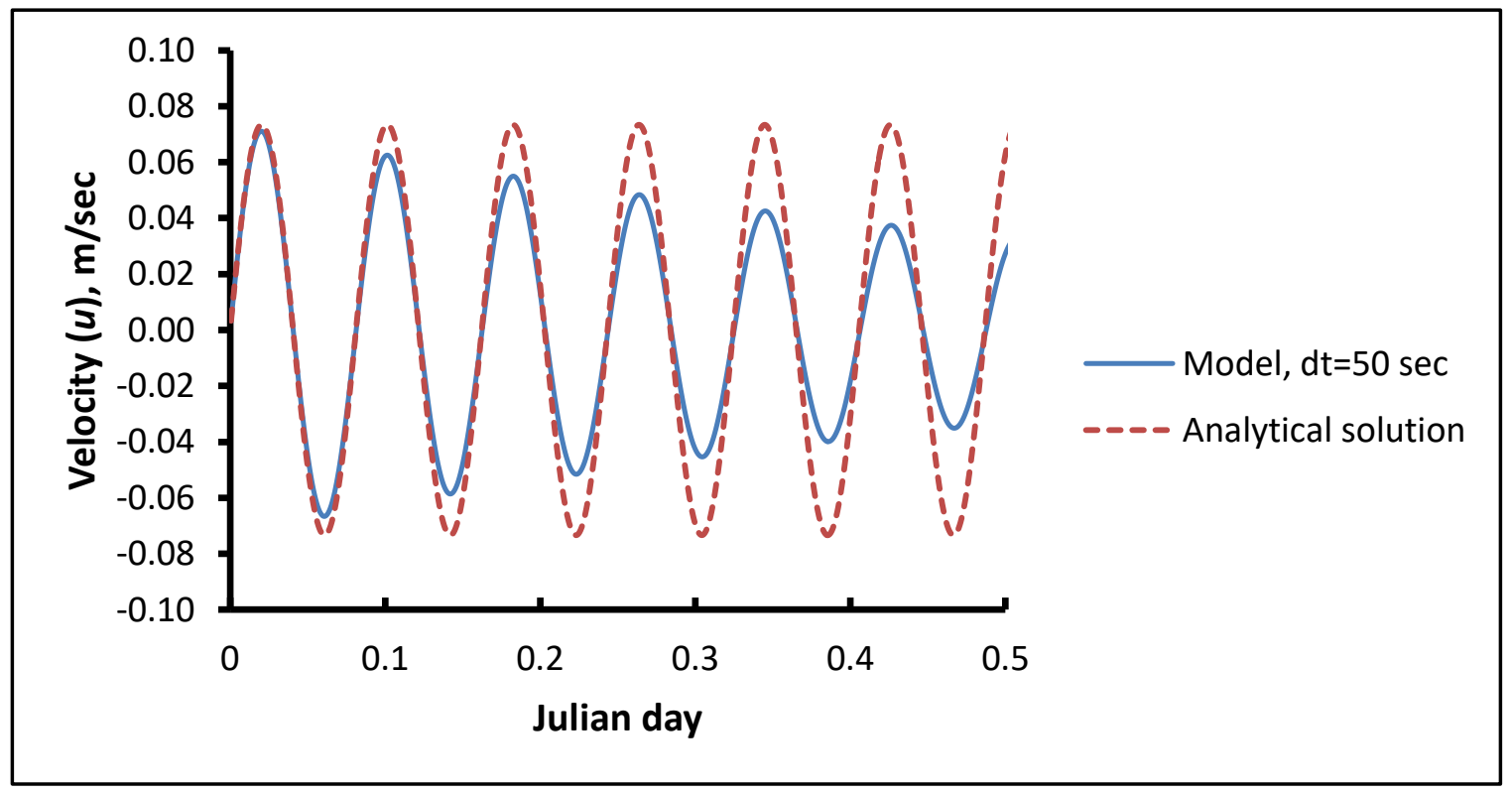

Figure 3-8. Comparison in the longitudinal velocity (u) between the model results and the analytical solution for the seiching basin near the left boundary $(i=3, j=3$, and $k=k t=3)$, $\Delta \mathrm{t}=\mathbf{5 0}$ sec 


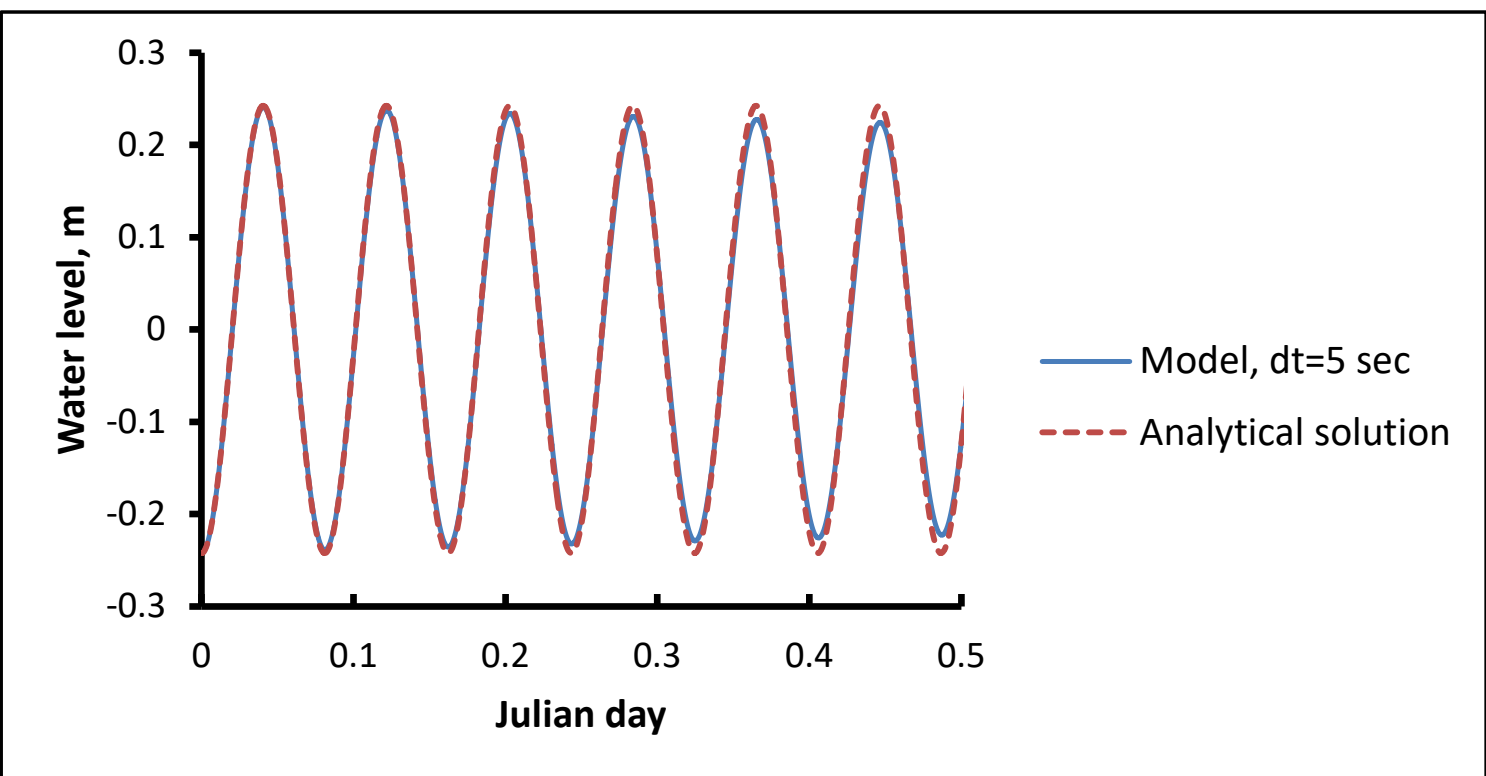

Figure 3-9. Comparison in the water level $(\eta)$ between the model results and the analytical solution for the seiching basin near the left boundary $(i=3, j=3$, and $k=k t=3), \Delta \mathrm{t}=5 \mathrm{sec}$

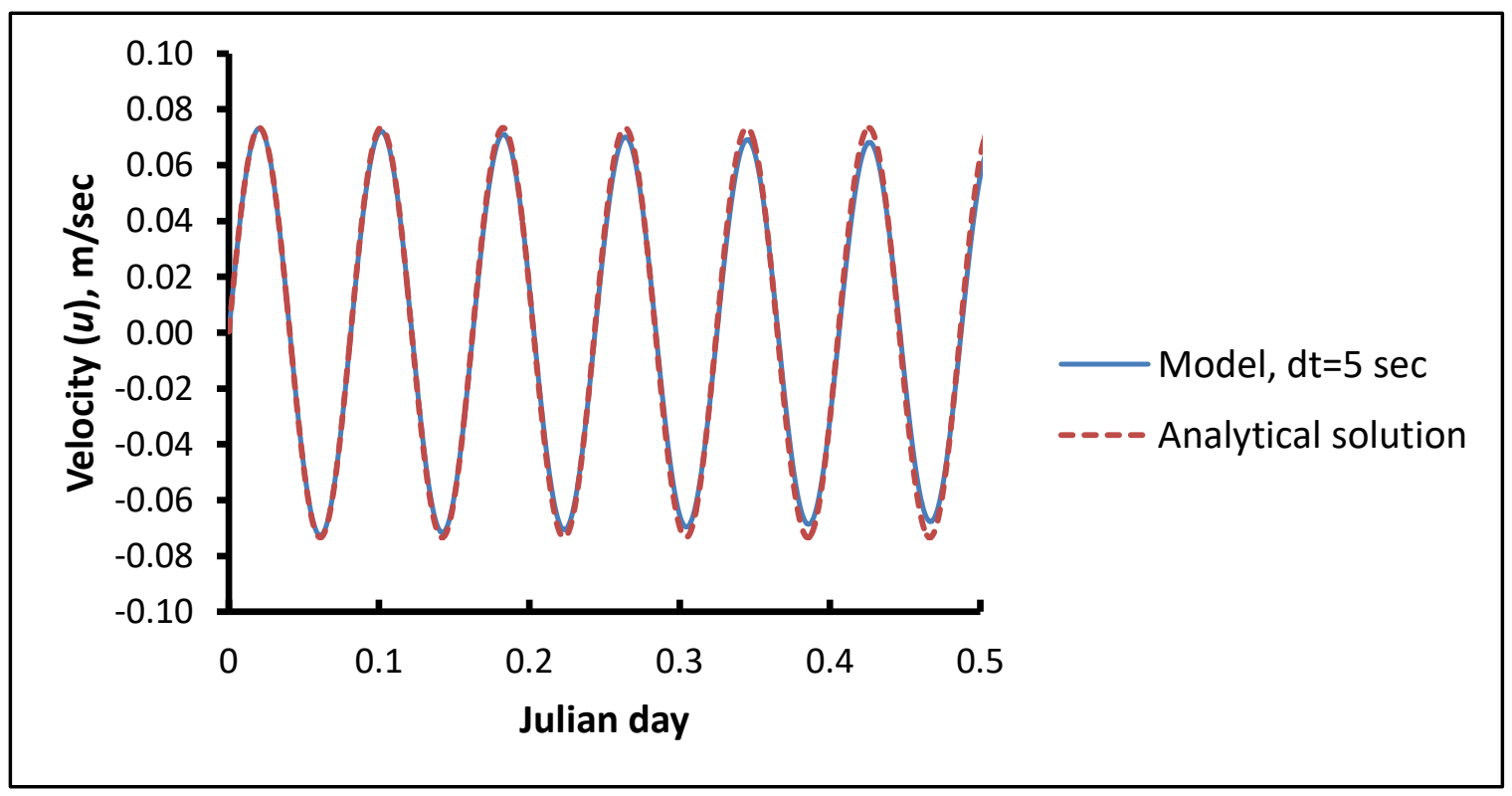

Figure 3-10. Comparison in the longitudinal velocity $(u)$ between the model results and the analytical solution for the seiching basin near the left boundary $(i=3, j=3$, and $k=k t=3), \Delta t=5$ sec 


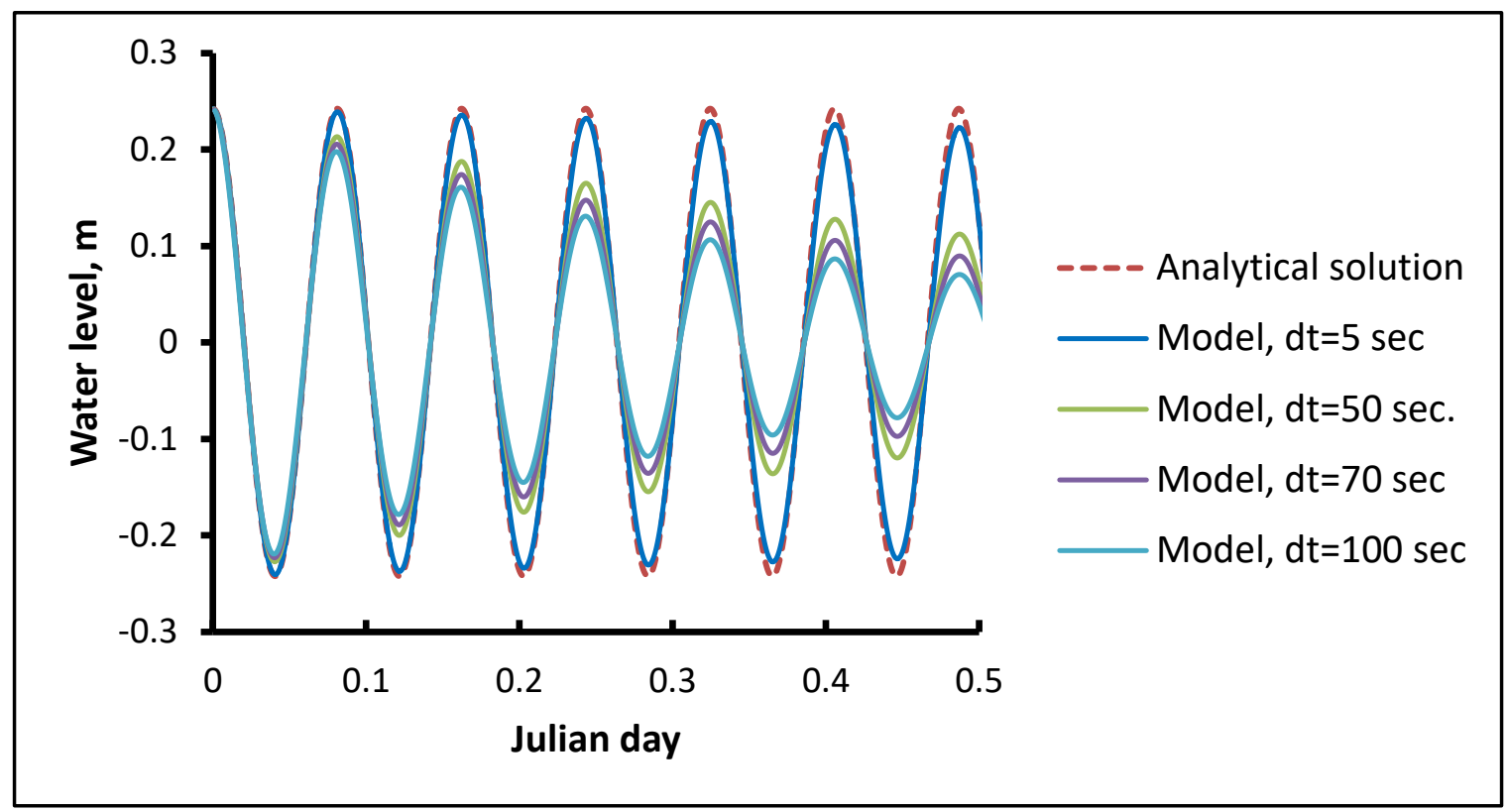

Figure 3-11. Dumping effect on the computed water level wave using different time steps for the seiching basin near the right boundary $(i=19, j=3$, and $k=k t=3)$

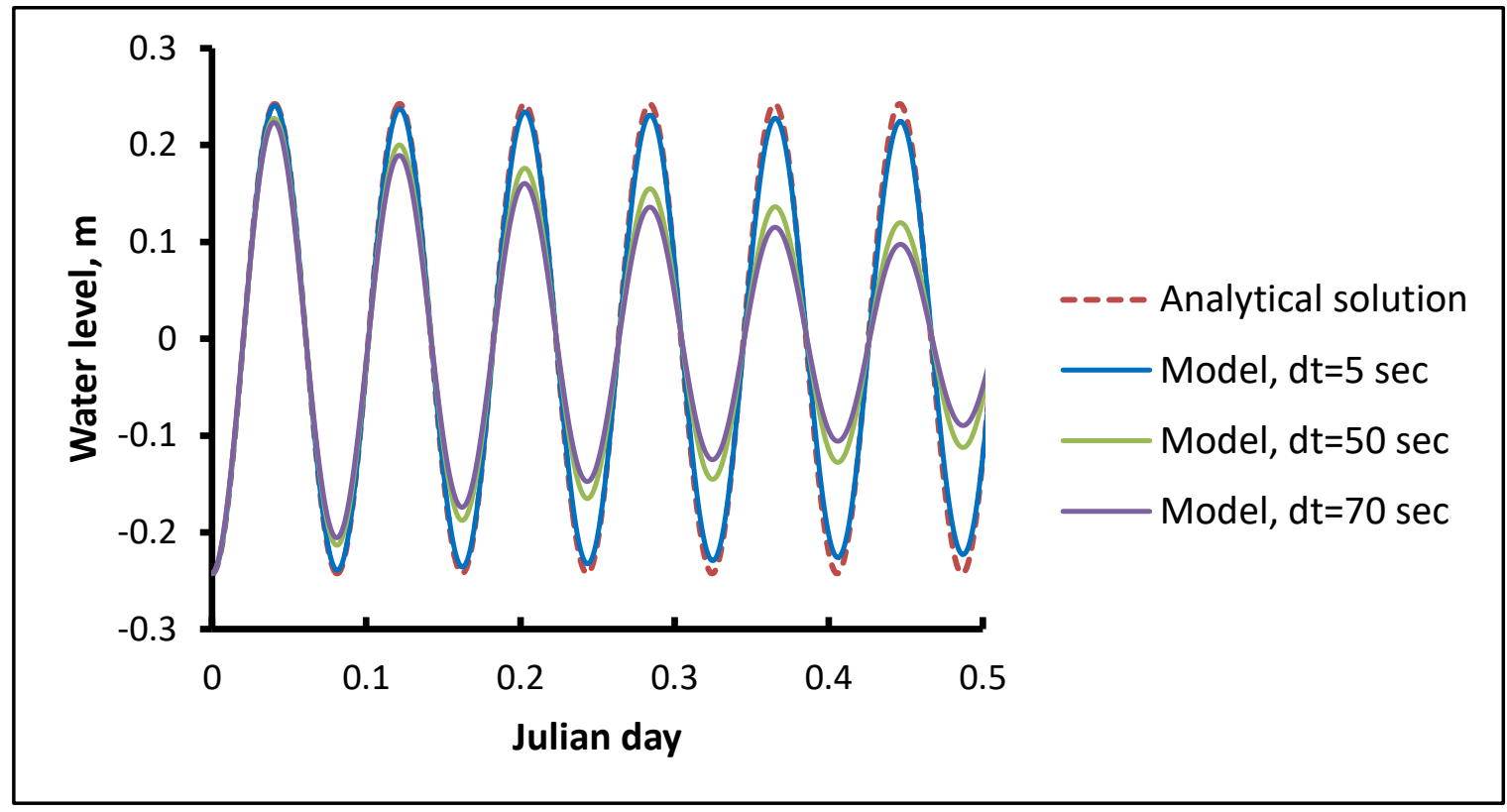

Figure 3-12. Dumping effect on the computed water level wave using different time steps for the seiching basin near the right boundary $(i=19, j=3$, and $k=k t=3)$ 


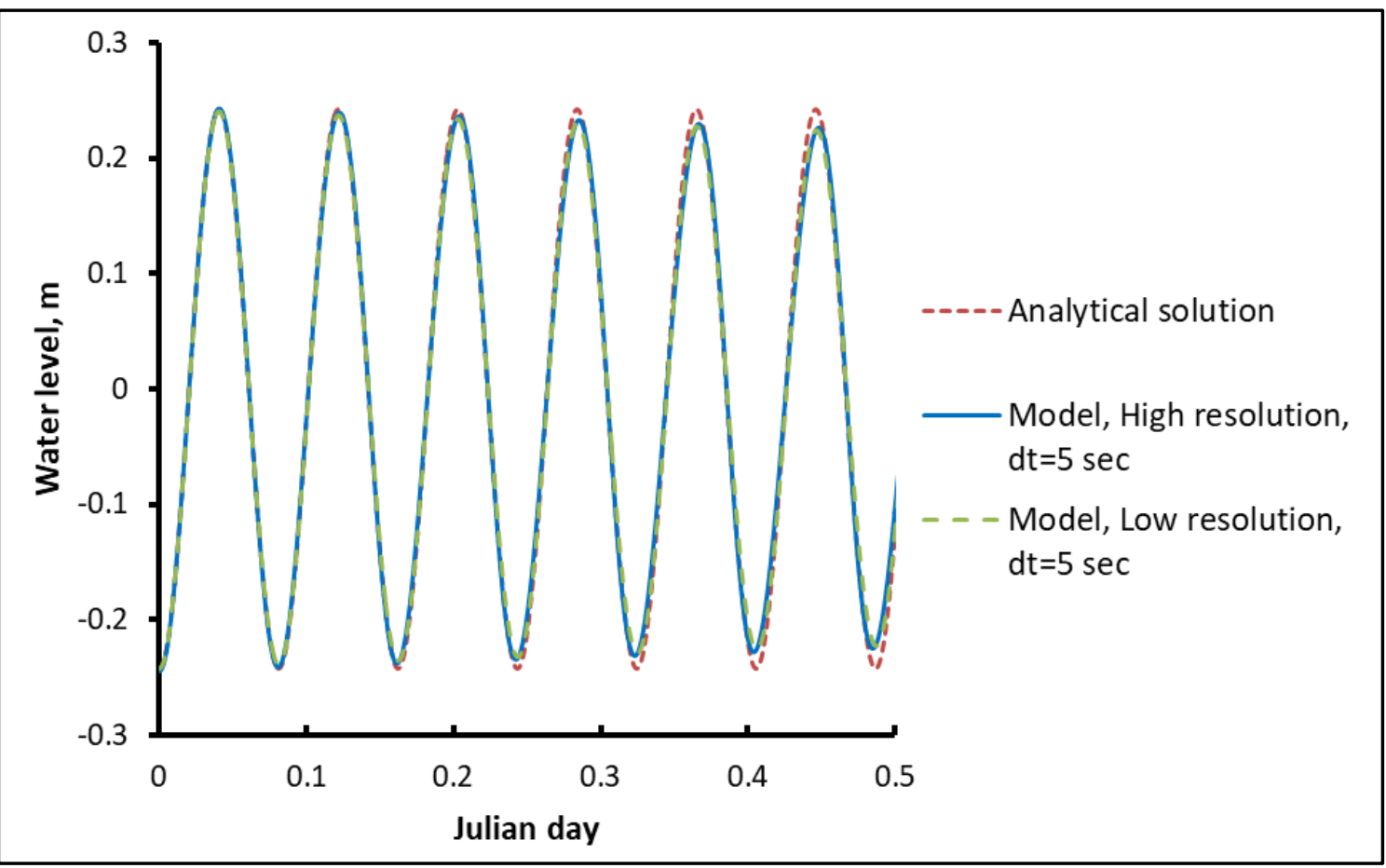

Figure 3-13. Comparison in water level wave using two spatial resolutions at the same time step

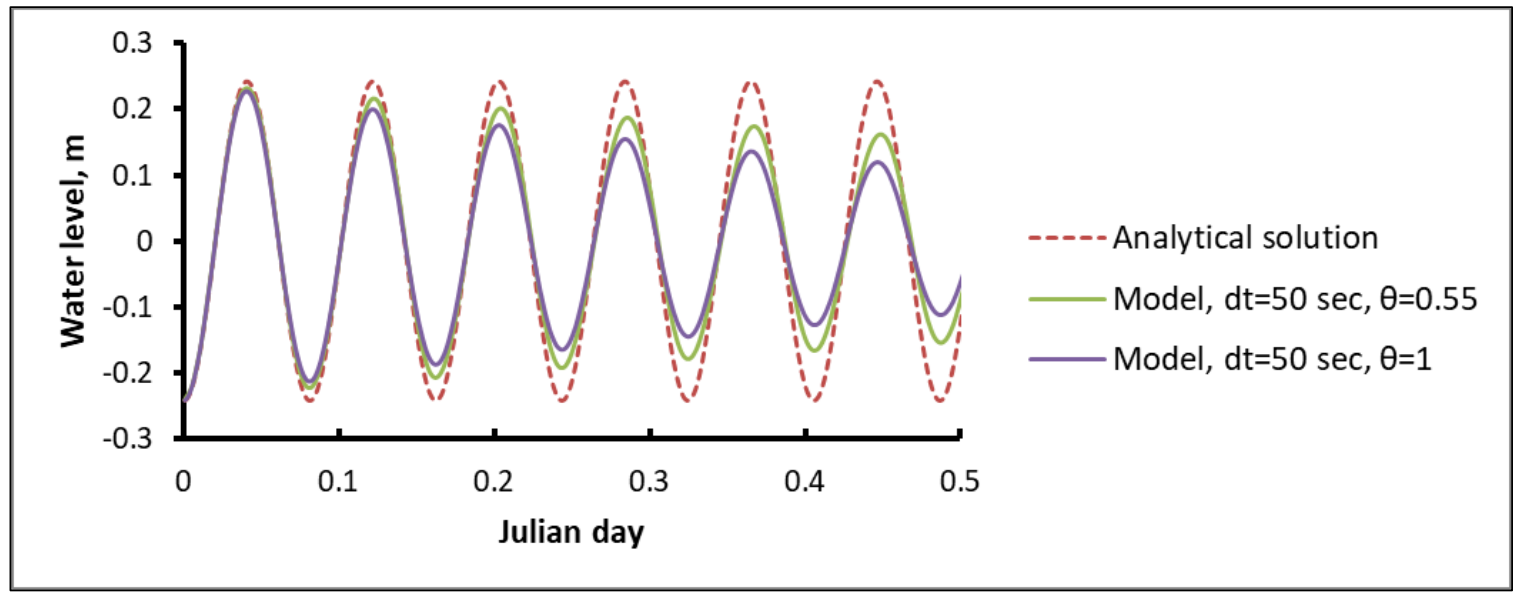

Figure 3-14. Effect of the degree of implicitness on damping rate of the computed water level wave for the seiching basin near the left boundary $(i=3, j=3$, and $k=k t=3), \Delta t=50$ sec 


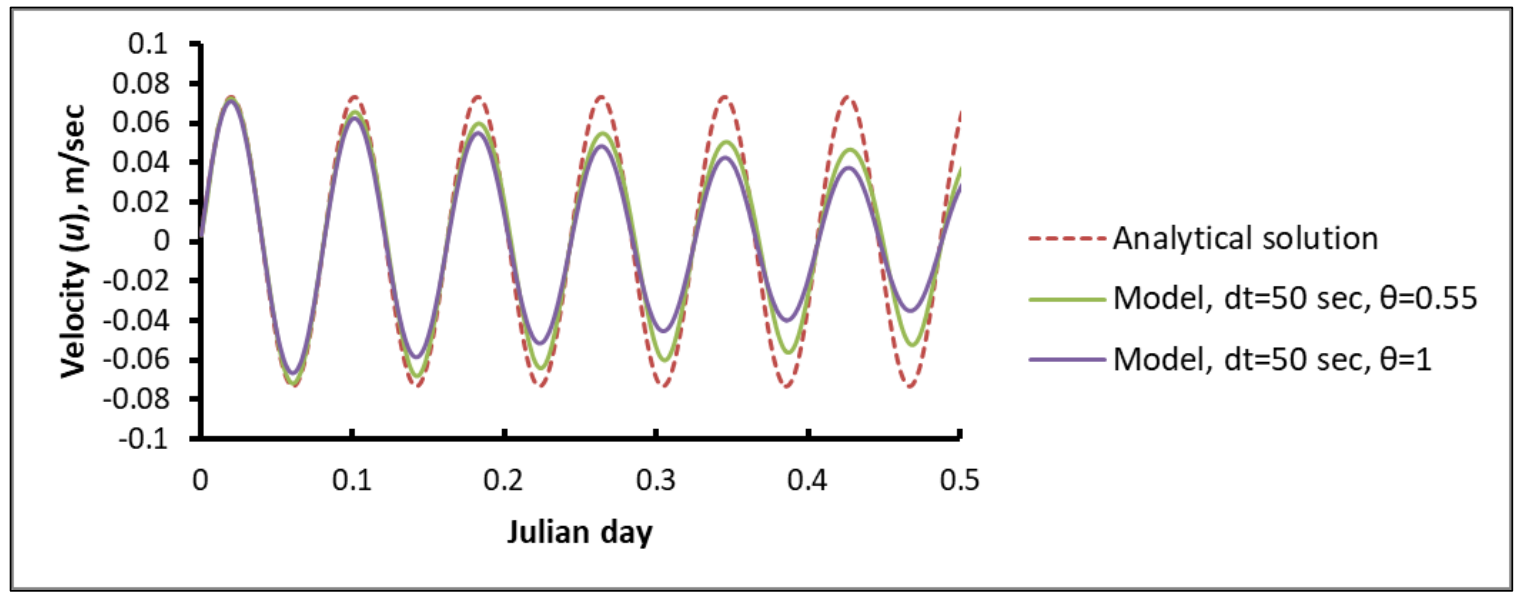

Figure 3-15. Effect of the degree of implicitness on damping rate of the computed velocity wave for the seiching basin near the left boundary $(i=3, j=3$, and $k=k t=3), \Delta t=50$ sec

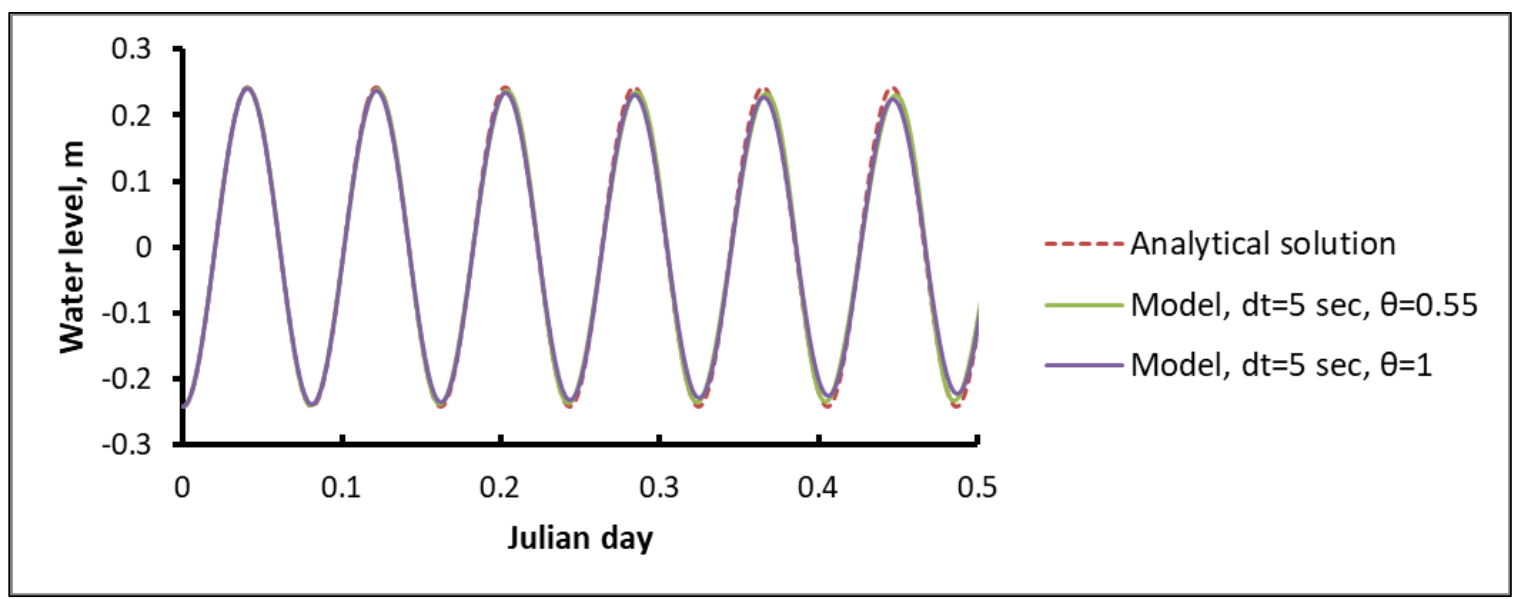

Figure 3-16. Effect of the degree of implicitness on damping rate of the computed water level wave for the seiching basin near the left boundary $(i=3, j=3$, and $k=k t=3), \Delta t=5$ sec 


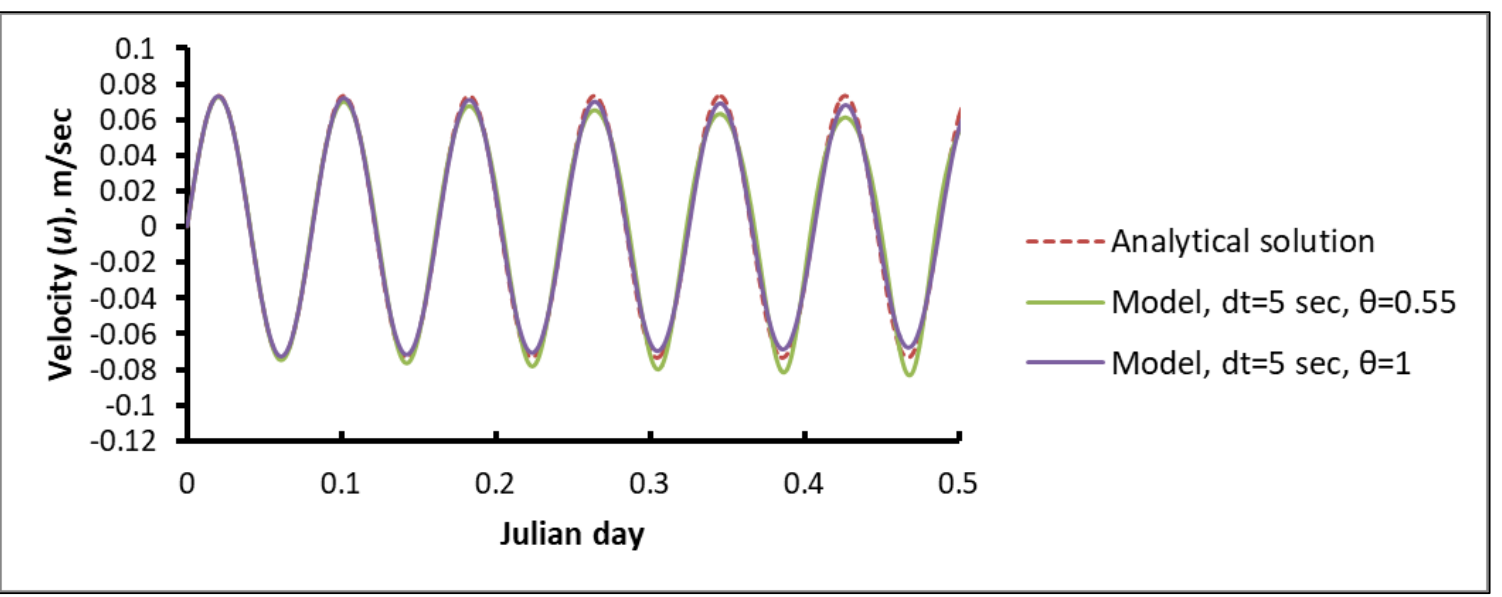

Figure 3-17. Effect of the degree of implicitness on damping rate of the computed velocity wave for the seiching basin near the left boundary $(i=3, j=3$, and $k=k t=3), \Delta t=5$ sec

\subsection{Test 2: Free Water Surface Response to Wind-Induced Flow in a Closed \\ Rectangular Basin}

To evaluate the influence of wind shear stress $\tau_{s}$ on the numerical solution in test case 1 , we added the surface shear stress to the X-Momentum equation that governs the seiching basin. Then, the governing equations of this test are:

The free surface equation:

$$
\frac{\partial \eta}{\partial t}+\frac{\partial u H}{\partial x}=0
$$

The X-Momentum equation:

$$
\frac{\partial u}{\partial t}+g \frac{\partial \eta}{\partial x}=\frac{\tau_{s}}{\rho_{\circ} H}
$$

If we considered $x=0$ is the center of the basin as shown in Figure 3-18, in which $L=2 b$, and suddenly a constant wind starts hitting the flat water surface, $\eta=0$, in the 
positive $x$-direction and continues blowing with time, the analytical solution for the water elevation was given by Wells (2002a):

$$
\begin{aligned}
\eta_{(x, t)}=\frac{u_{*}^{2}}{g H} x- & \frac{8 b u_{*}^{2}}{\pi^{2} g H}\left[\cos \left(\frac{\pi \sqrt{g H} t}{2 b}\right) \sin \left(\frac{\pi x}{2 b}\right)-\frac{1}{9} \cos \left(\frac{3 \pi \sqrt{g H} t}{2 b}\right) \sin \left(\frac{3 \pi x}{2 b}\right)\right. \\
+ & \left.\frac{1}{25} \cos \left(\frac{5 \pi \sqrt{g H} t}{2 b}\right) \sin \left(\frac{5 \pi x}{2 b}\right)-\cdots\right]
\end{aligned}
$$

Where $u_{*}$ is the surface shear velocity.

Note that the $x$-axis is defined differently between the one dimensional governing equations related to the test 2 and the present three-dimensional model. Therefore, a coordinate transformation was done in the code to the $x$-axis of the one-dimensional governing equations analytical solution to match the three-dimensional model numerical solution.

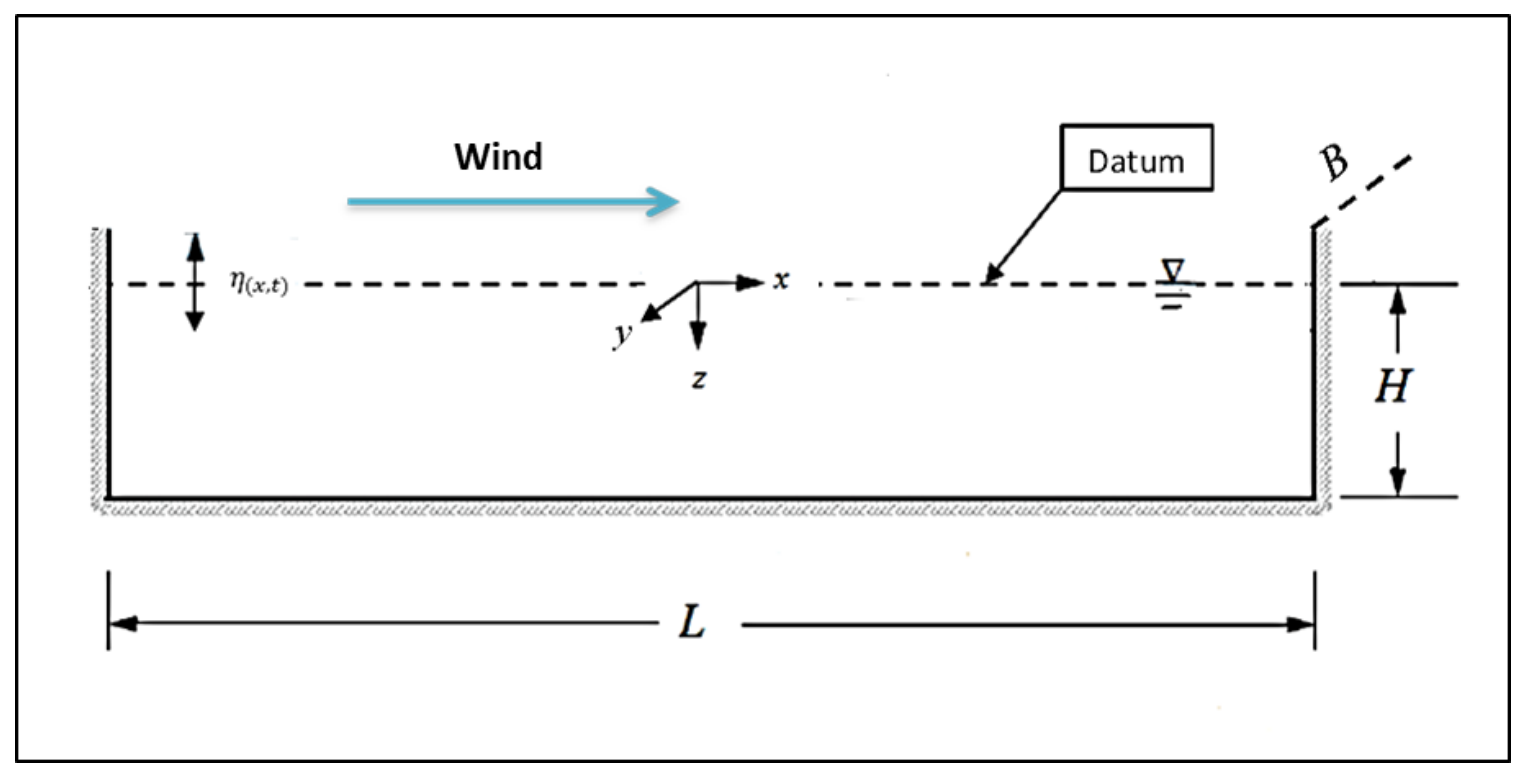

Figure 3-18. Seiching basin for the test 2 
The code was run with constant wind of $2 \mathrm{~m} / \mathrm{sec}$ at $10 \mathrm{~m}$ height above the water surface at $t=0$. A comparison in the water level $(\eta)$ between the model results and the analytical solution near the left boundary of the seiching basin is shown in Figure 3-19. The model showed good results in following the surface wave signal of the analytical solution. Therefore, by comparing test case 1 and 2, under the effect of wind there is no extra damping to the surface wave compared to the case where there is no wind. Figure 3-20 shows the wind effect on the water surface level upstream and downstream of the basin. In this case the waves of opposite directions at both the left and right end are similar to those of test case 1 . The upstream wave though has positive amplitude which is greater than the negative amplitude of the downstream wave, implying the water surface has a positive slope in the wind direction.

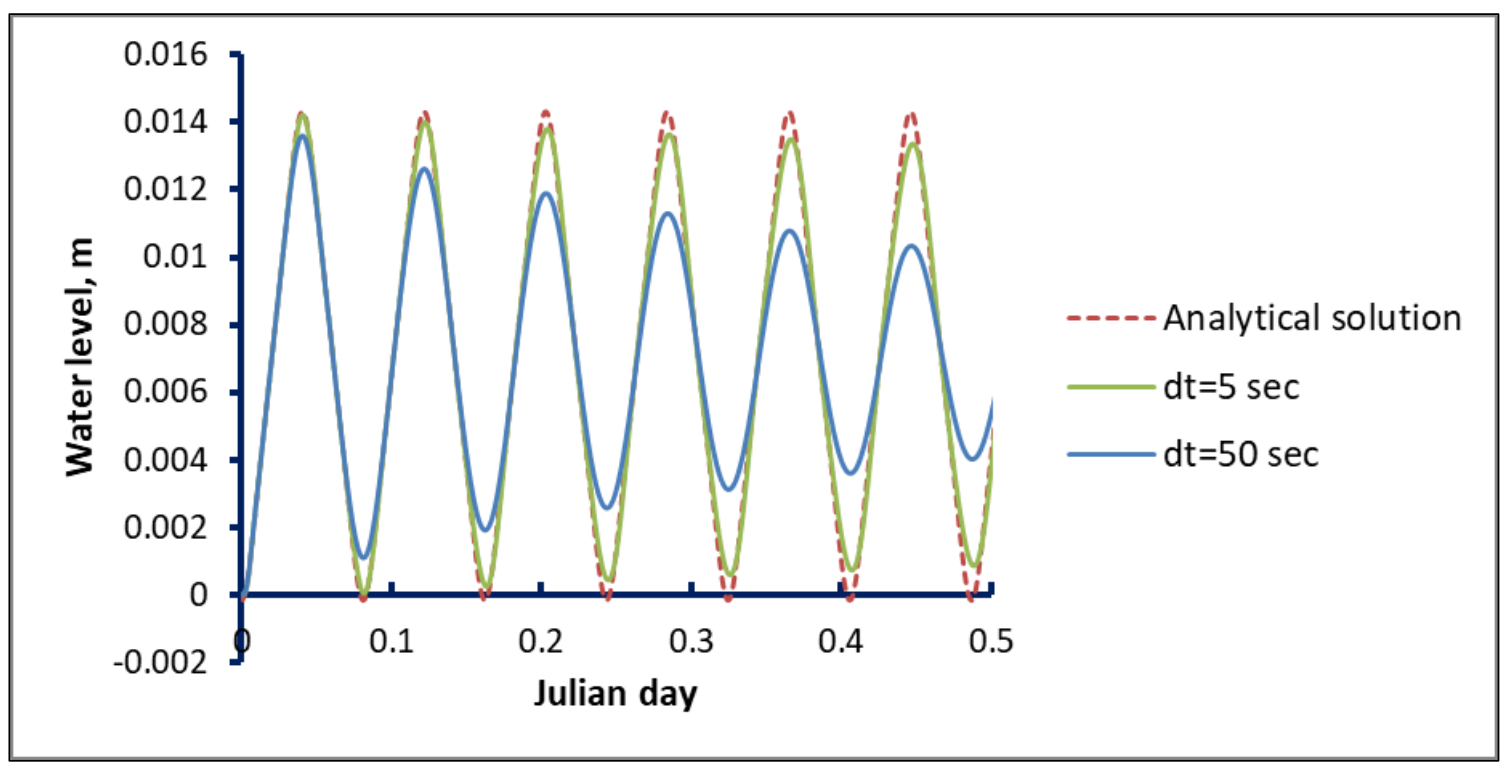

Figure 3-19. The computed water level under the wind effect using different time steps for the seiching basin near the left boundary $(i=3, j=3$, and $k=k t=3)$ 


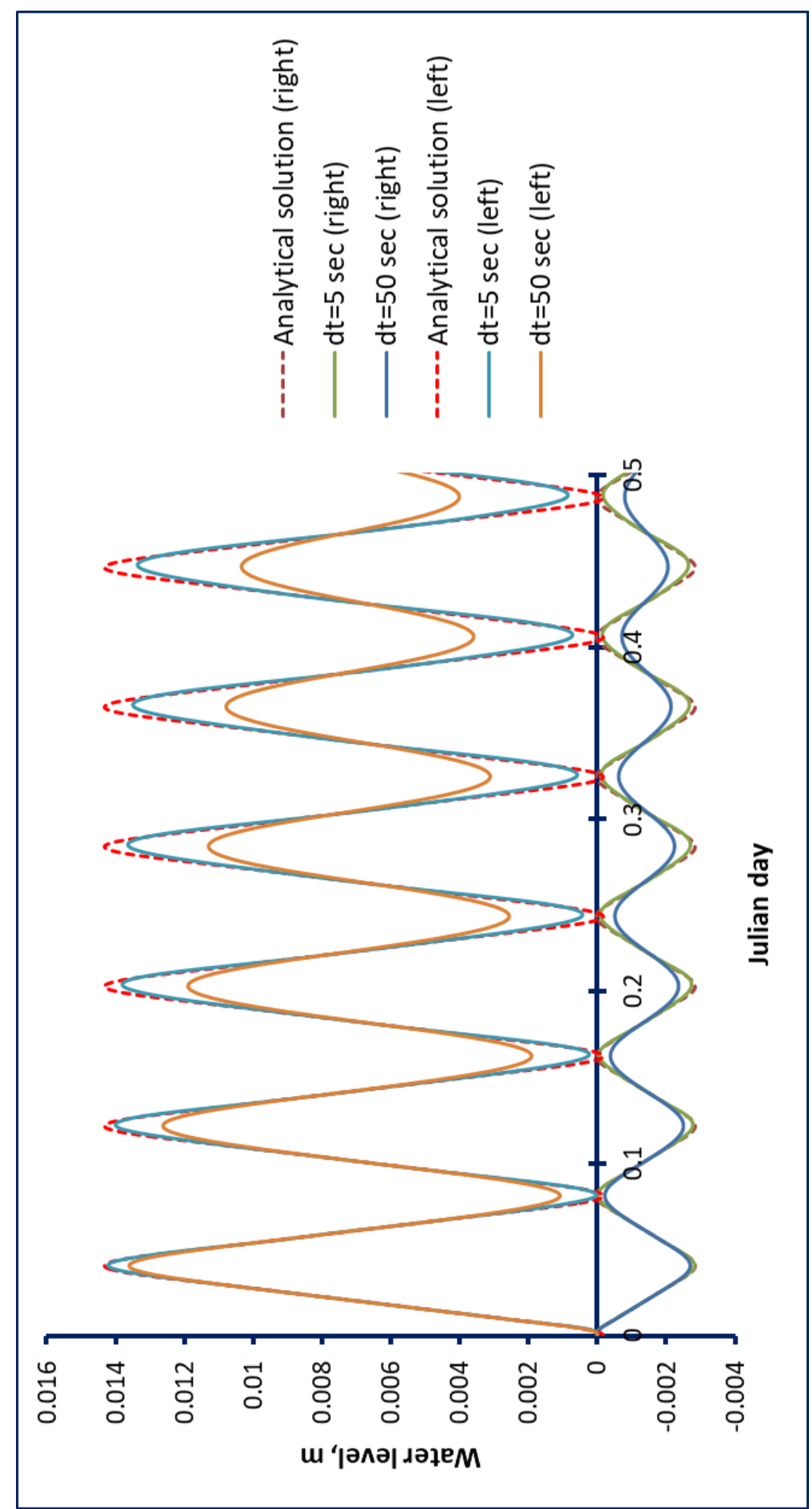

Figure 3-20. The computed water level under the wind effect using different time steps for the seiching basin near the left and right boundaries $(i=3$ and $i=19, j=3$, and $k=k t=3$ ) 


\subsection{Test 3: Velocity Profile Response to the Wind Induced Flow in a Closed Rectangular Basin}

The surface shear stresses due to the wind blowing on the waterbody are transferred vertically by vertical eddy viscosity resulting in a velocity profile in which the water surface flows in the direction of the wind downstream and then it hits the boundary and circulates back upstream through the bottom layers. Different models are available to represent the analytical velocity profile. One of these models is a model developed by Hansen (1975). The analytical solution is in term of error function,

$$
\frac{u}{u_{*}}=6.65\left[1-\operatorname{erf}\left(\frac{z}{0.267 u_{*}}\right)\right]
$$

Where $u$ is the longitudinal velocity over time at a depth of $z$ below the water surface, see Figure 3-21.

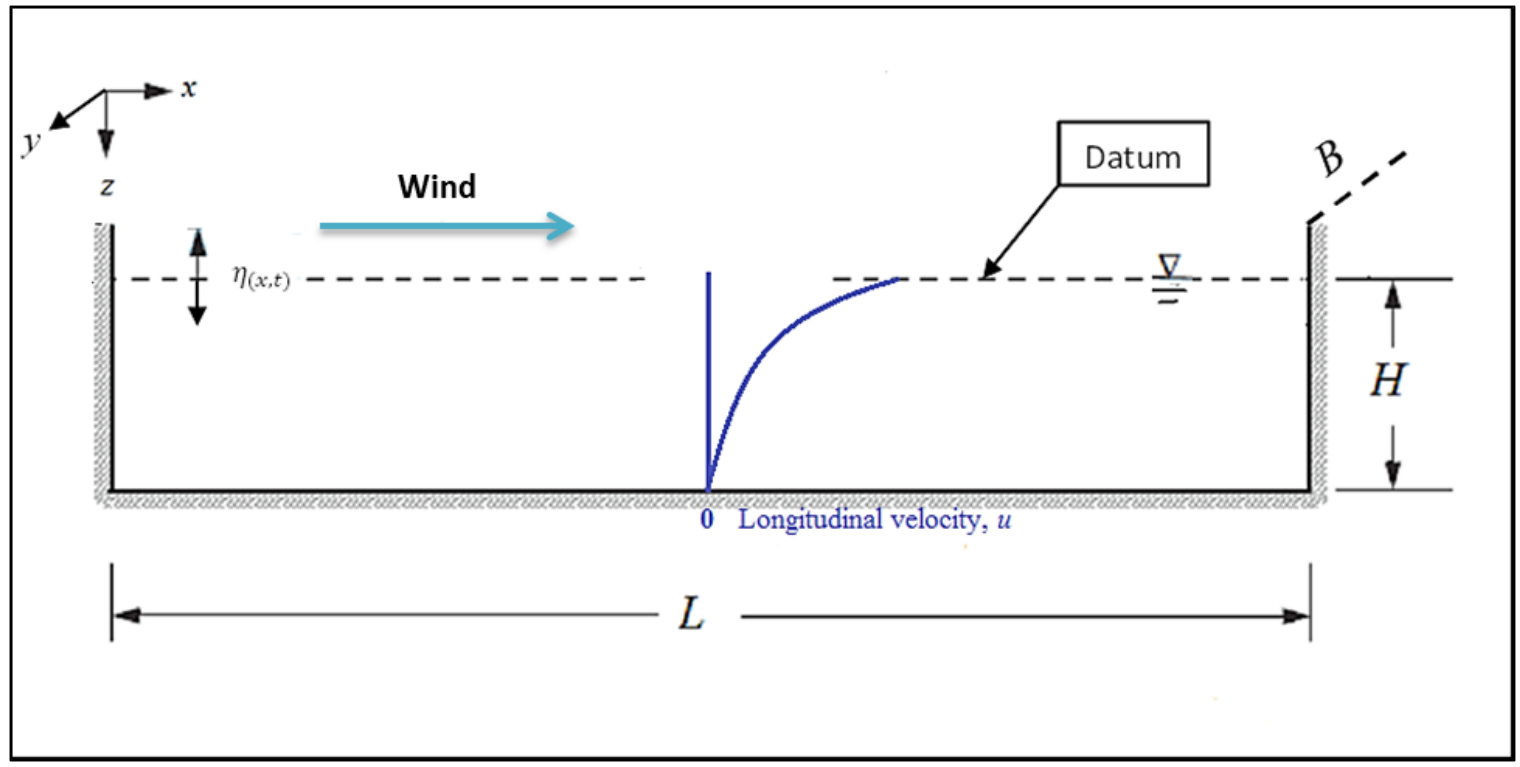

Figure 3-21. Test 3 closed rectangular basin 
Since the solution is based on assuming there is a balance between the rate of change of the longitudinal velocity and its vertical diffusion only, we need to run the code for a short period of time when the change in the water surface level can be considered negligible to agree with the analytical solution. Also, we need to turn off the horizontal advection, horizontal diffusion, and Coriolis force. Using a constant vertical eddy viscosity over depth $v_{t}=\frac{1}{28} u_{*}^{2} t$ (Scott A. Wells, 2002a) and wind of $10 \mathrm{~m} / \mathrm{sec}$ in the positive $x$-direction, the code was run for $1000 \mathrm{sec}$. Figure 3-22 shows the computed and analytical velocity profile under the effect of wind induced flow in the middle of the basin where the effect of circulation and boundaries are negligible. The model gave very good agreement with the analytical solution. This agreement with the analytical solution is important because in lakes and reservoirs, wind induced currents determine the vertical mixed thermal structure. The vertical mixed thermal structure then can affect water quality including algae growth dynamics which through self-shading can affect the density regime. Hence, the necessity of having a 3D model that solves the hydrodynamic equations at the same time level as the water quality equations. 


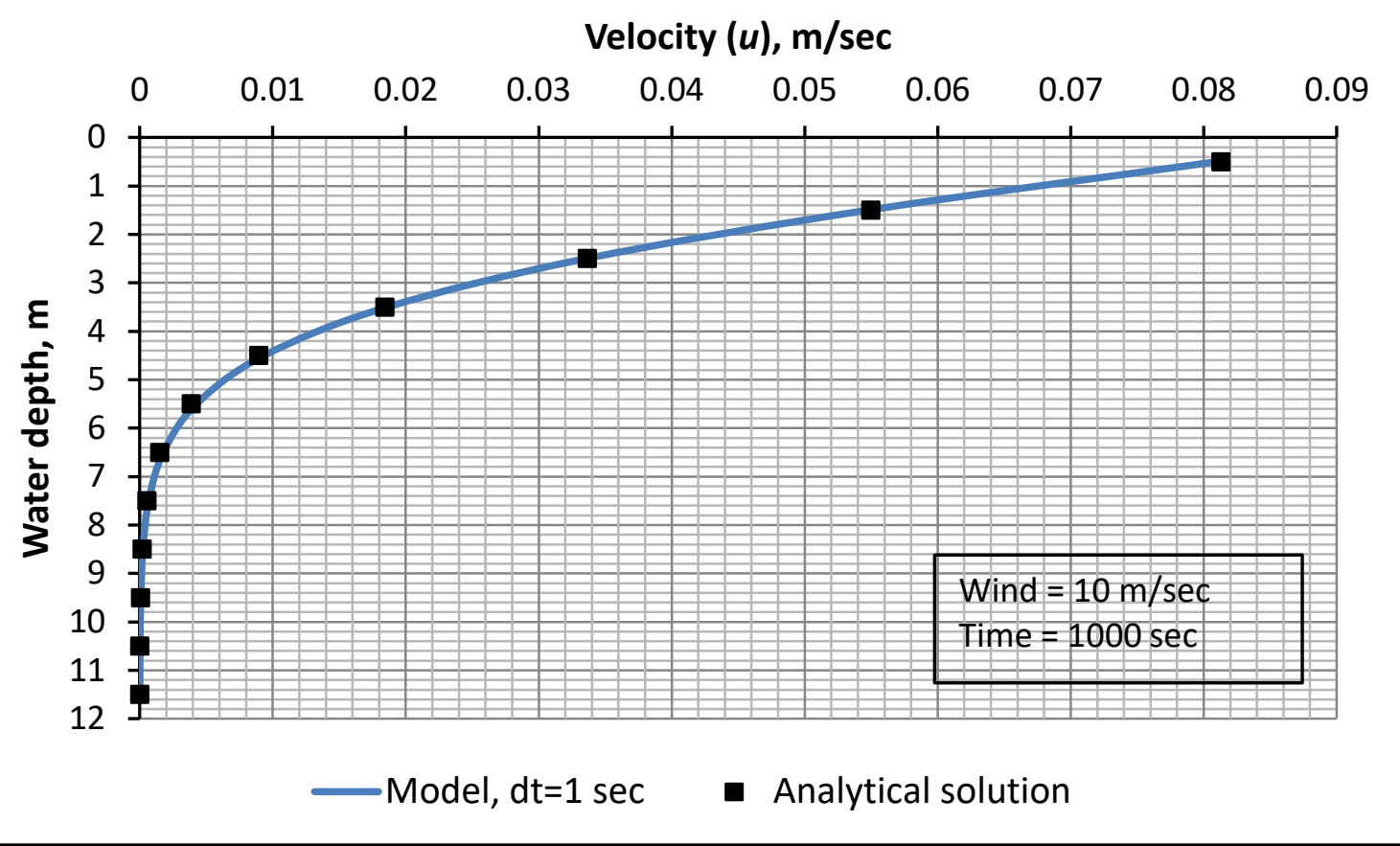

Figure 3-22. The computed and analytical velocity profile under the effect of wind induced flow in the middle of the seiching basin at time $=1000 \mathrm{sec}$

\subsection{Test 4: Volume Balance}

The volume balance was performed by comparing the water volume in the model domain during a time period with the water volume entering and leaving the same domain during the same period of time.

Let $\mathrm{Vol}$ be the accumulated water volume in the model domain over time. Then,

$$
V o l=V o l_{\text {initial }}+V o l_{\text {in }}-V o l_{\text {out }}
$$

Where:

Vol $_{\text {initial }}=$ the initial water volume within the domain,

$V o l_{\text {in }}=$ the accumulated water volume entering the domain, and 
$\mathrm{Vol}_{\text {out }}=$ the accumulated water volume leaving the domain.

Thus, the error over time can be calculated as follows:

$$
\% \text { Error }=\frac{a b s\left(\text { Vol }- \text { Vol }_{\text {internal }}\right)}{\text { Vol }_{\text {internal }}} \times 100
$$

Where Vol $_{\text {internal }}$ is the water volume within the domain at any time during the simulation period.

A subroutine was added to the model to check the volume preservation by calculating \% Error at every time step. A lower \% Error represents more accurate model predictions. The error should reach a constant value with time and should not grow with time. If \% Error grows with time exponentially, this implies that the model goes unstable (blows up). Two tests implementing the volume balance check were performed. One of these tests examined the volume balance over a rectangular domain, and the other test evaluated the volume balance over an irregular domain. Both tests were performed over a period of 100 days based on the same real meteorological data, calculated solar short radiation, and constant inflow and outflow, The meteorological data are shown in Figure 3-23 to Figure 3-27. 


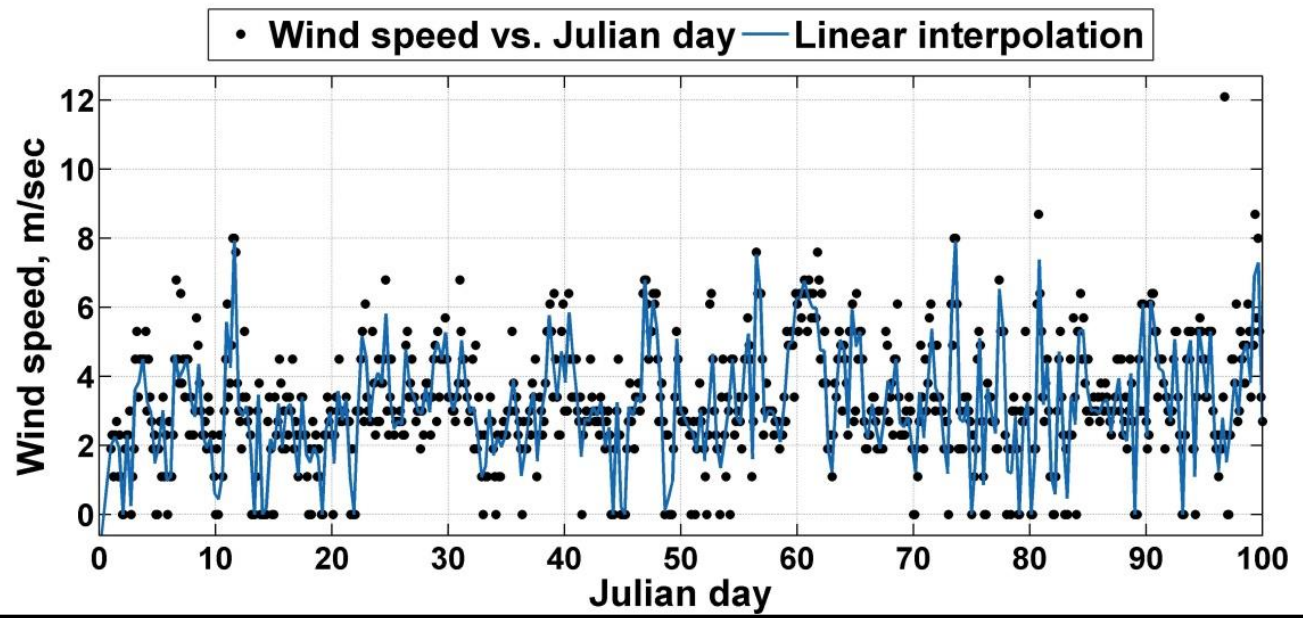

Figure 3-23. Test 4 wind speed input data

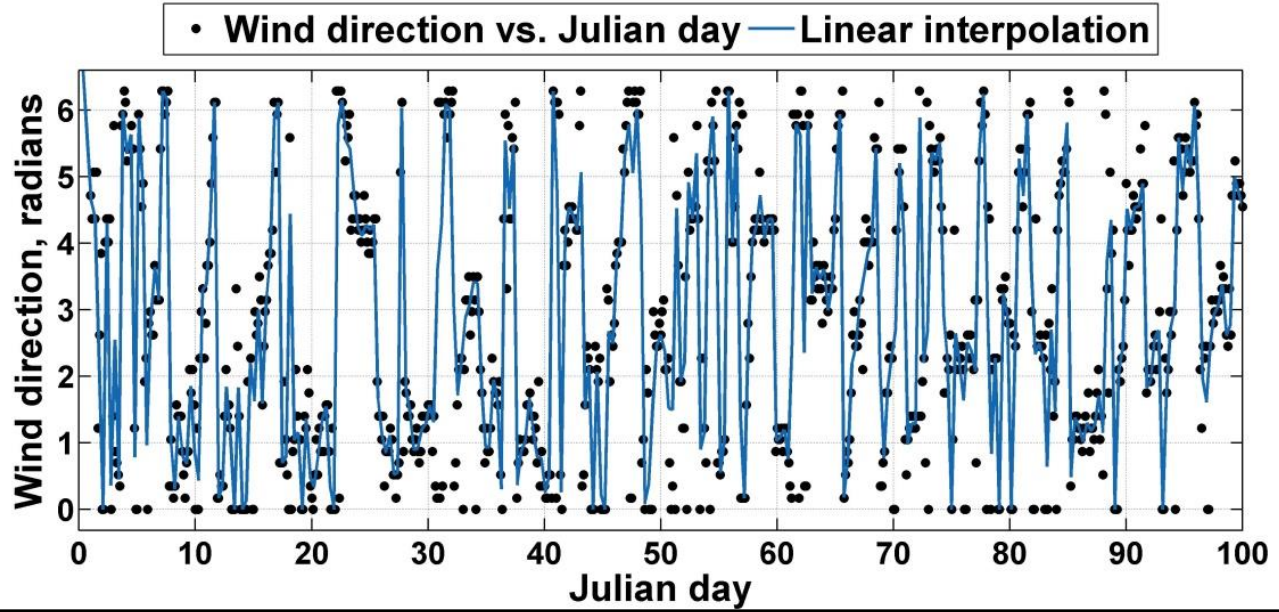

Figure 3-24. Test 4 wind direction input data 


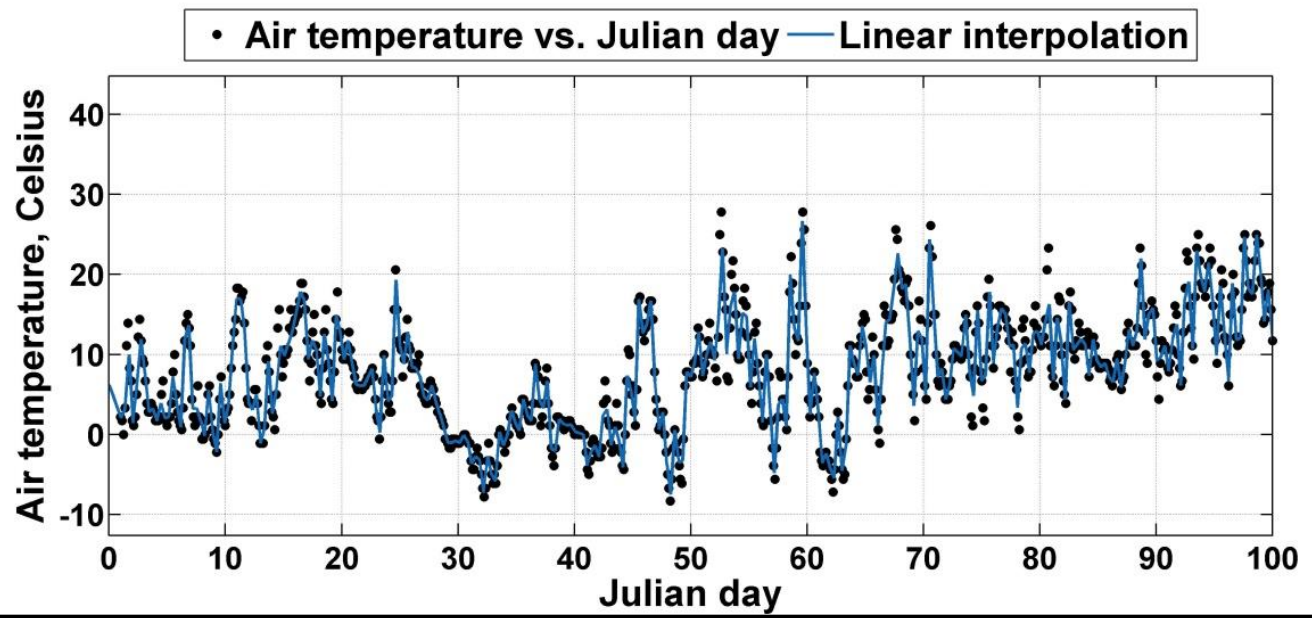

Figure 3-25. Test 4 air temperature input data

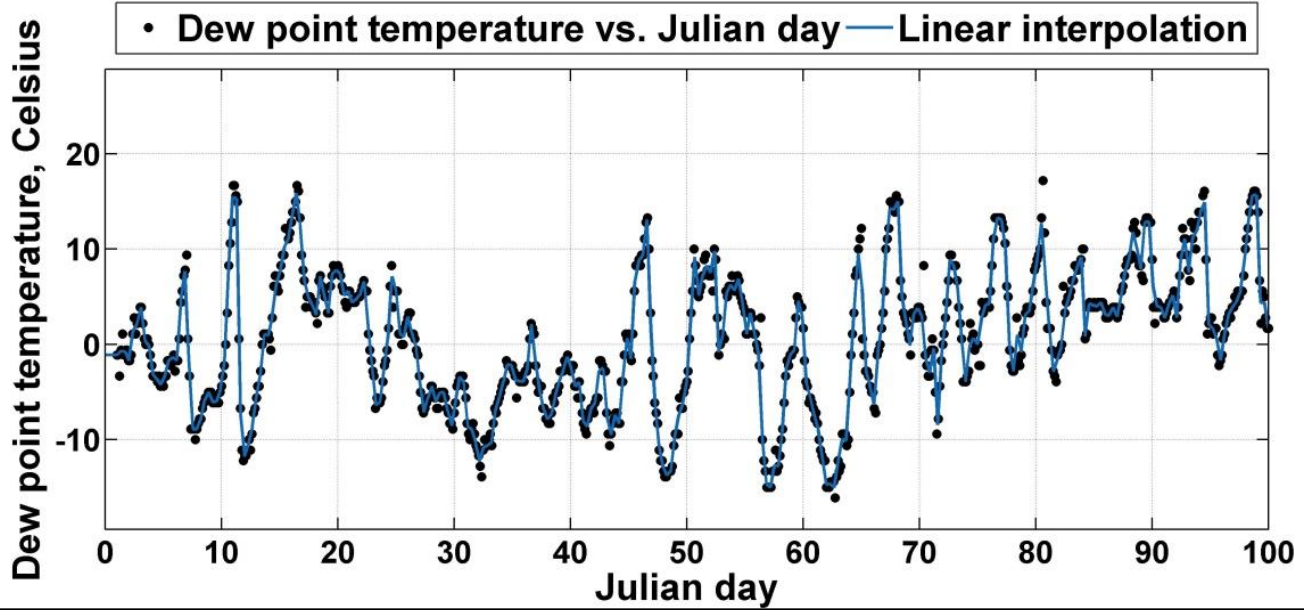

Figure 3-26. Test 4 dew point input data 


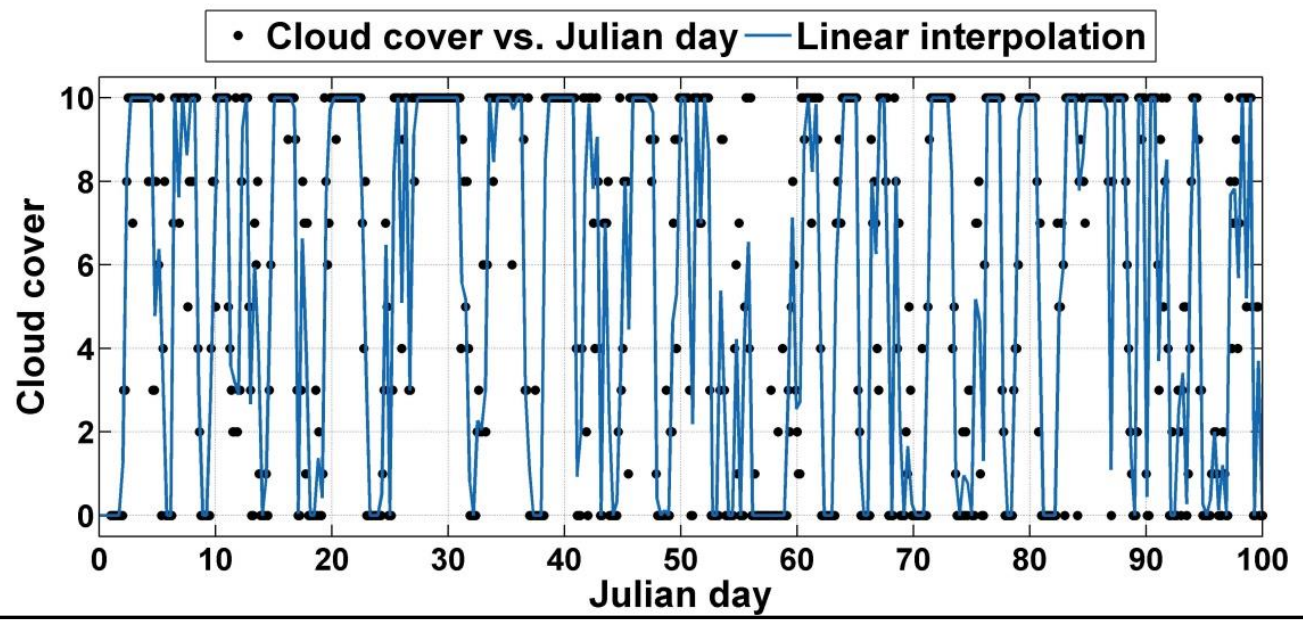

Figure 3-27. Test 4 cloud cover input data

\subsubsection{Irregular Physical Domain}

The physical domain was divided into computational cells of $1000 \times 500 \times 1(x, y, z) \mathrm{m}$ and oriented perpendicular to the north direction as shown in Figure 3-28, in which there are bends at the boundaries to check how the model catches the flow field variability. The code of test case 4 was run without assuming a frictionless fluid, with the Coriolis force, with wind variable in magnitude and direction at $10 \mathrm{~m}$ height above the water surface, with a constant inflow and outflow of $0.8 \mathrm{~m}^{3} / \mathrm{sec}$, and with variable water temperature over time by solving the heat transport equation. Additionally, the adding/subtracting layers algorithm (Cole \& Wells, 2017) was turned on to examine the surface layer thickness over the simulation period.

Using a time step of $35 \mathrm{sec}$ and $\theta=1$, the code was run for the simulation period.

Figure 3-29 presents the model predictions of the surface velocity field at Julian day 100. 
The model results showed good performance in following the bends at the boundaries. Furthermore, the volume balance error gave good agreement in preserving volume in which the percent error reached a constant low value over time as shown in Figure 3-30, which is a semi-log plot of the percent error with time. The corresponding water levels at three locations over time were as shown in Figure 3-31, denoting a very small change $(\cong$ $0.005 \mathrm{~m}$ ) in the surface layer thickness resulting from the free water surface waves.

The effect of the degree of implicitness on the accumulated error was evaluated by running the code using $\theta=0.5$ with the same inputs that were used with $\theta=1$. The results showed that using the semi-implicit scheme of $\theta=0.5$ produces less percent error than that by using $\theta=1$. Figure 3-32 shows the percent error after running the code for 100 day using two degrees of implicitness $(\theta=1$ and $\theta=0.5)$.

In addition and in order to make sure that the numerical answers do not depend on the grid resolution, a grid refinement was performed and the associated volume error was assessed. The code was run using $\theta=0.5$ with three horizontal grid resolutions $1000 \times 500$, $500 \times 500$, and $500 \times 125(x, y) \mathrm{m}$ in which the model was stable numerically. To maintain the stability, three different time steps were chosen to run the code because the refinement lowers the time step $(\Delta \mathrm{t})$. All resolutions were applied on the same initial water volume in Figure 3-28. Therefore, the initial water volume of the waterbody was fixed, while the grid resolution was varied. Figure 3-33 shows the percent error over time for the three considered grid resolutions, indicating that the error in volume has the same order of magnitude for the three resolutions. 


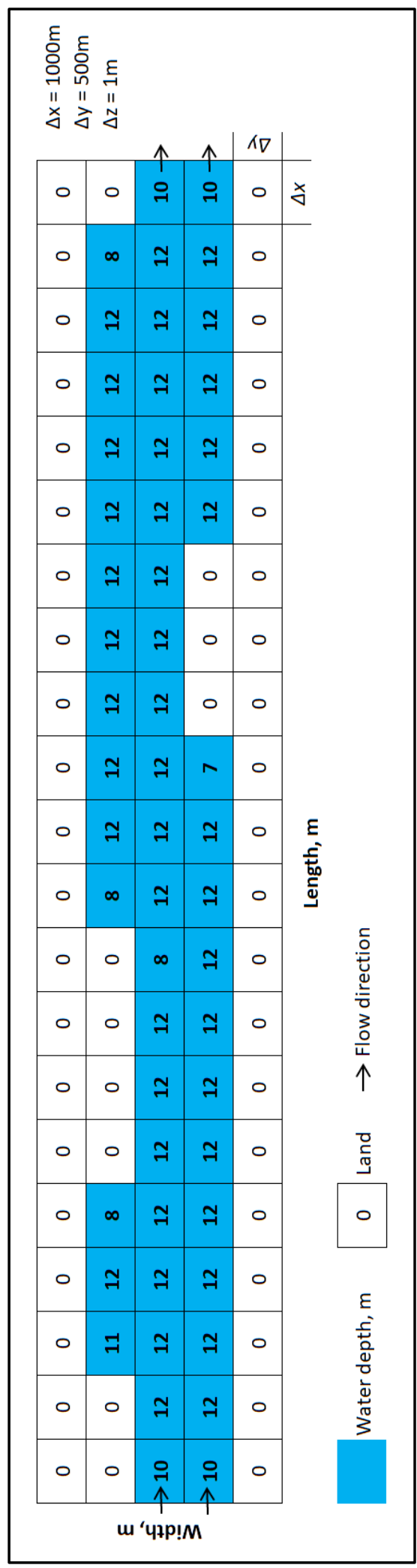

Figure 3-28. Test 4 irregular physical domain and the input bathymetry 122 


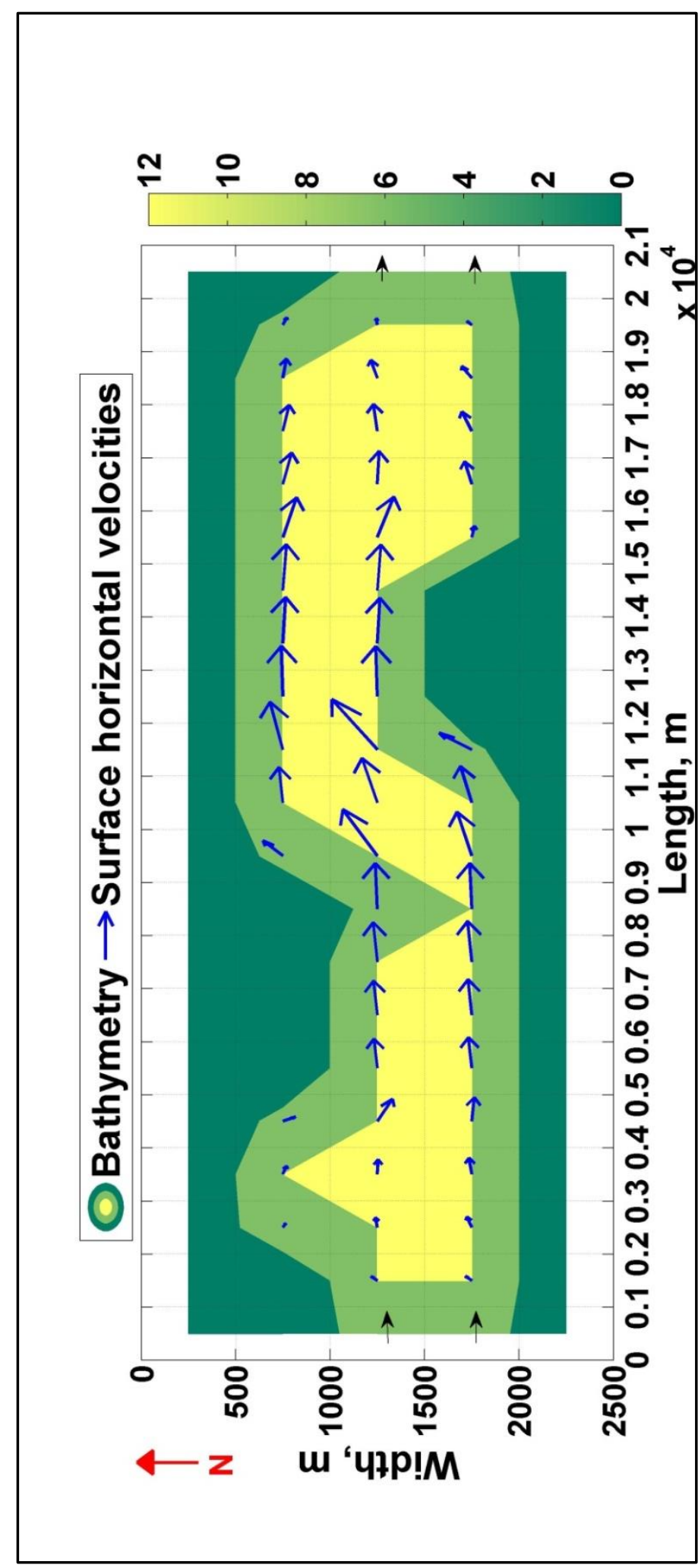

Figure 3-29. Test 4 surface velocity field for the irregular domain at Julian day 100 


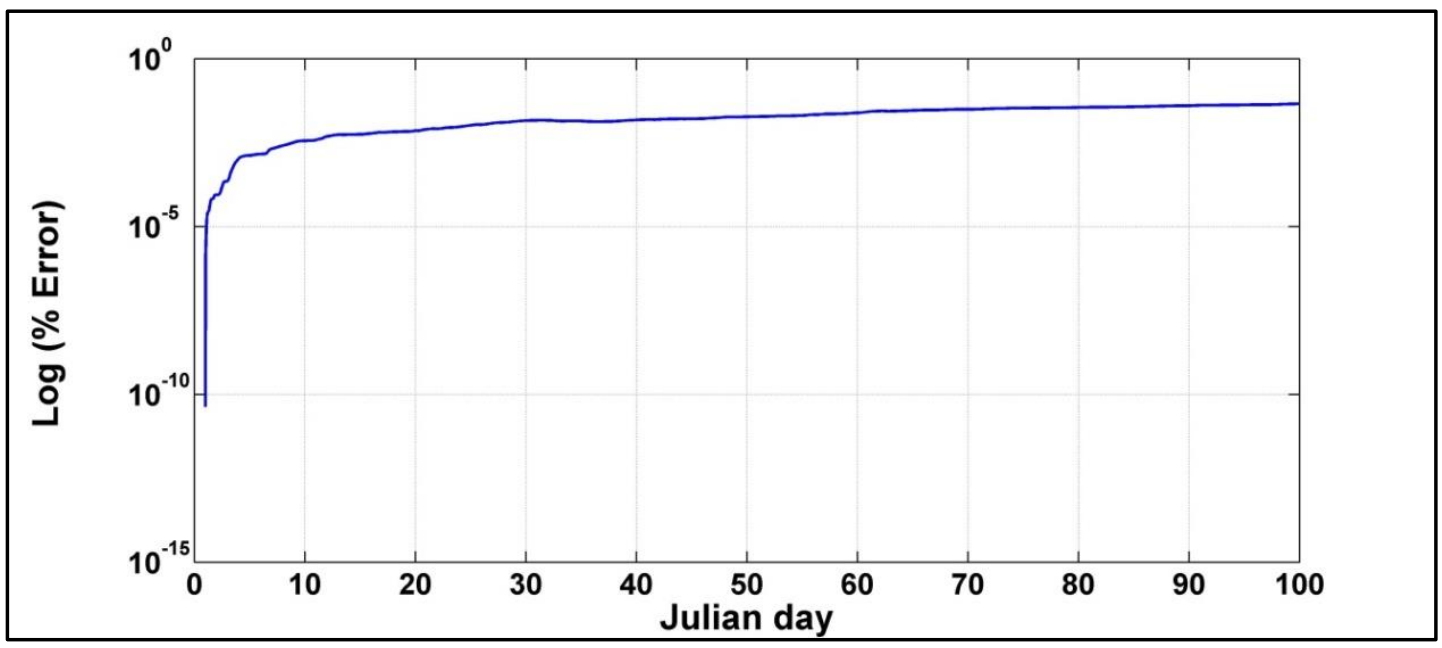

Figure 3-30. Test 4 volume balance for the irregular domain using $\theta=1$

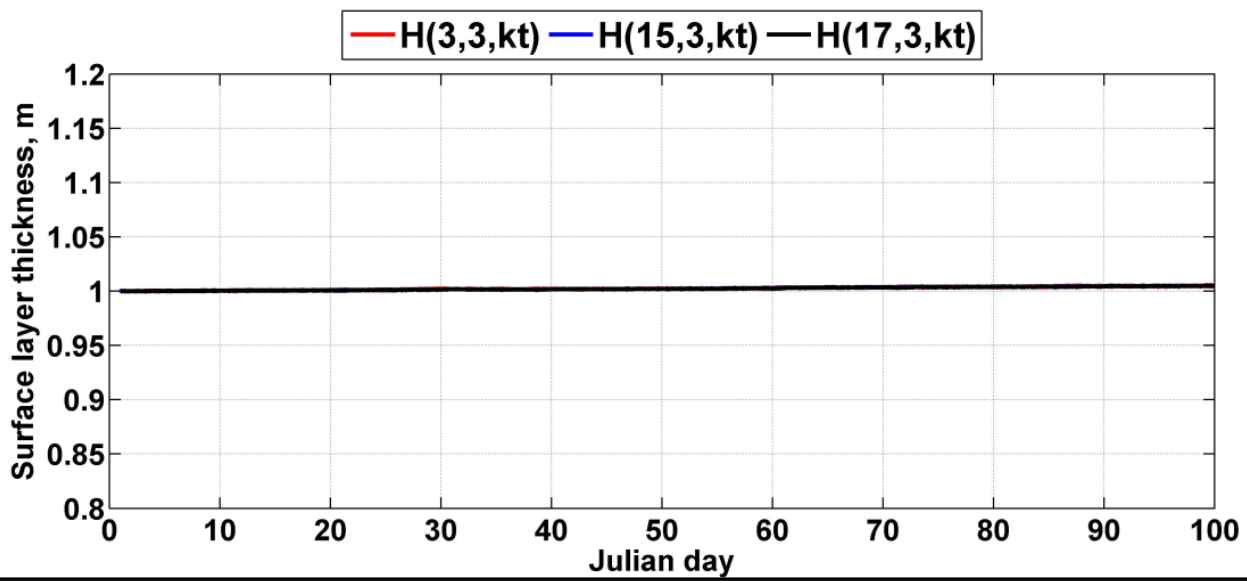

Figure 3-31. Test 4 surface layer thickness over time for the irregular domain using $\theta=1$

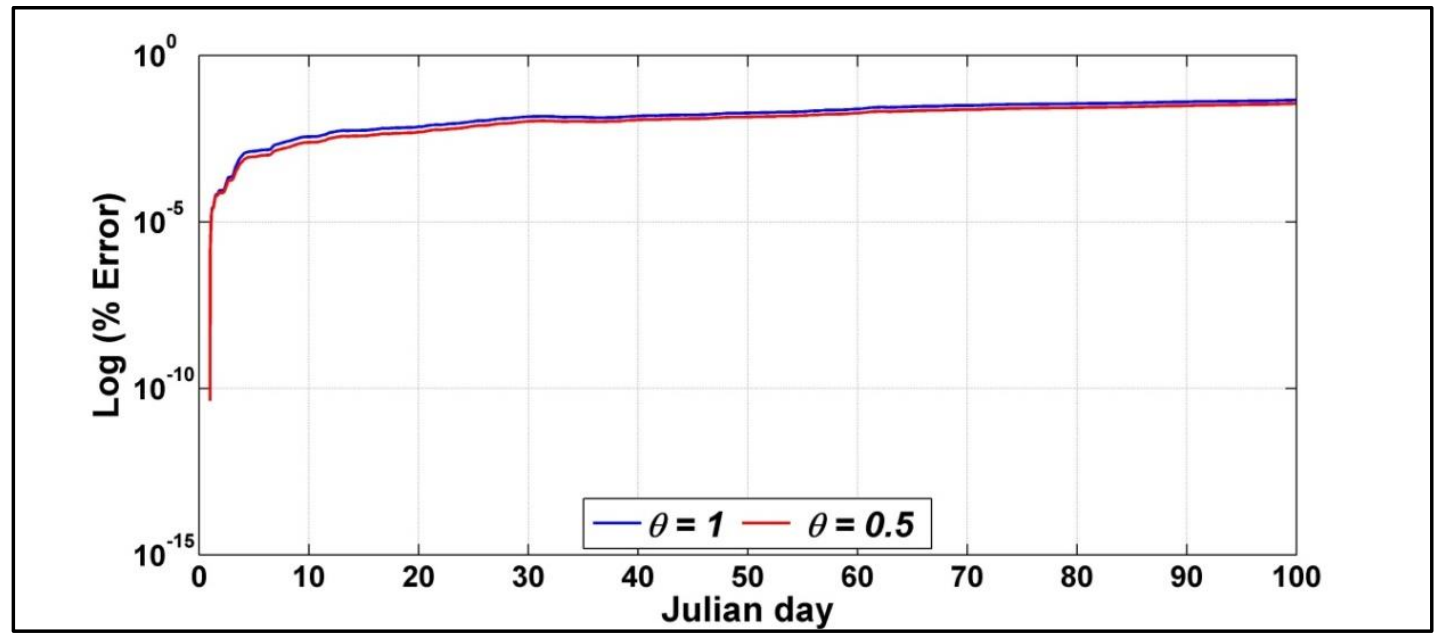

Figure 3-32. Test 4 the volume balance for the irregular domain using $\theta=1$ and $\theta=0.5$ 


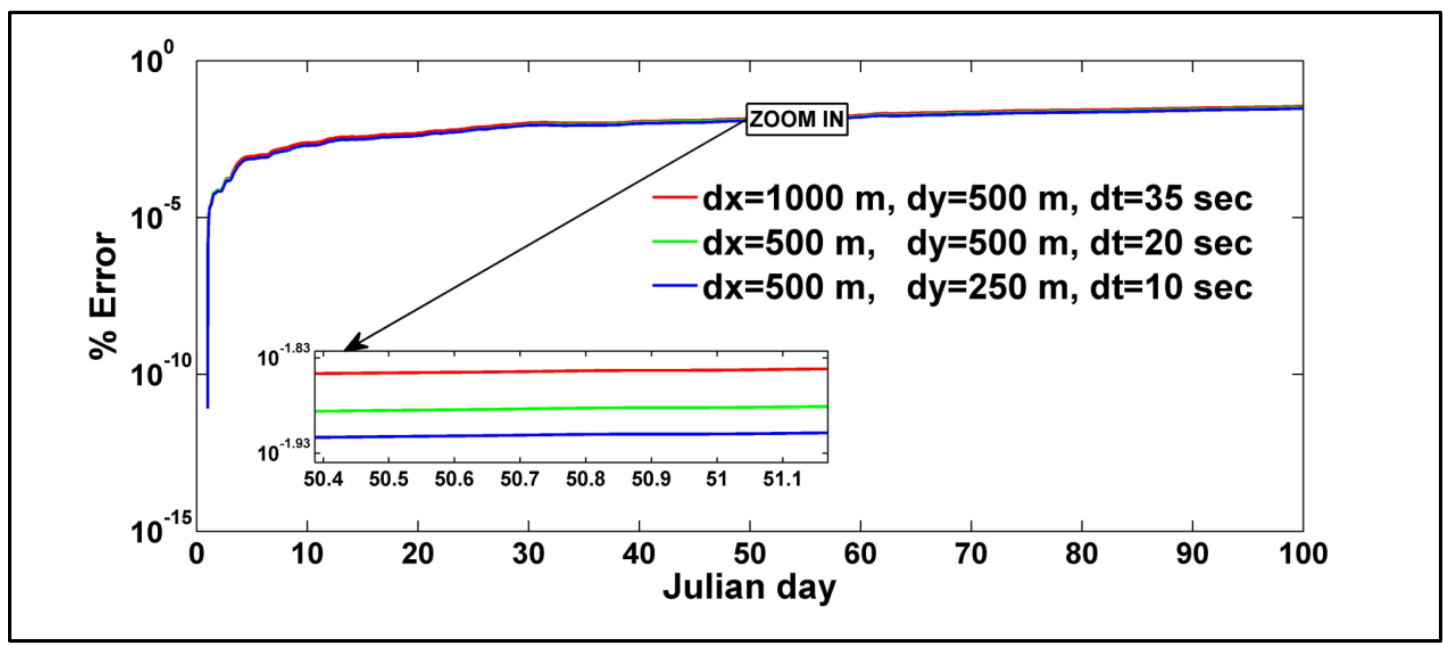

Figure 3-33. Test 4 the grid refinement 


\subsubsection{Rectangular Physical Domain}

This test was performed to check the volume balance for a fully rectangular domain as shown in Figure 3-34. The code was run using $\theta=1$ and the same input data that was used in the irregular domain test. The only difference between the two runs was the domain bathymetry. Figure 3-35 presents the surface velocity field at Julian day 100. Also, the model predictions of the percent error and the surface layer thickness over time are shown in Figure 3-36 and Figure 3-37, respectively. This test produced an error less than the irregular domain test, reflecting the effect of irregular domain on the volume conservation. Implementing the semi-implicit scheme was also performed in a similar way to the irregular physical domain test, and the results are shown in Figure 3-38. The test also showed that the degree of implicitness has less influence on the volume balance moving from an irregular boundary to a more uniform boundary. However, using the semi implicit scheme of $\theta=0.5$ helped reducing the accumulated error with time. The amount of reduction in percent error for the rectangular physical domain was less than that for the irregular physical domain due to the effect of flow uniformity. 


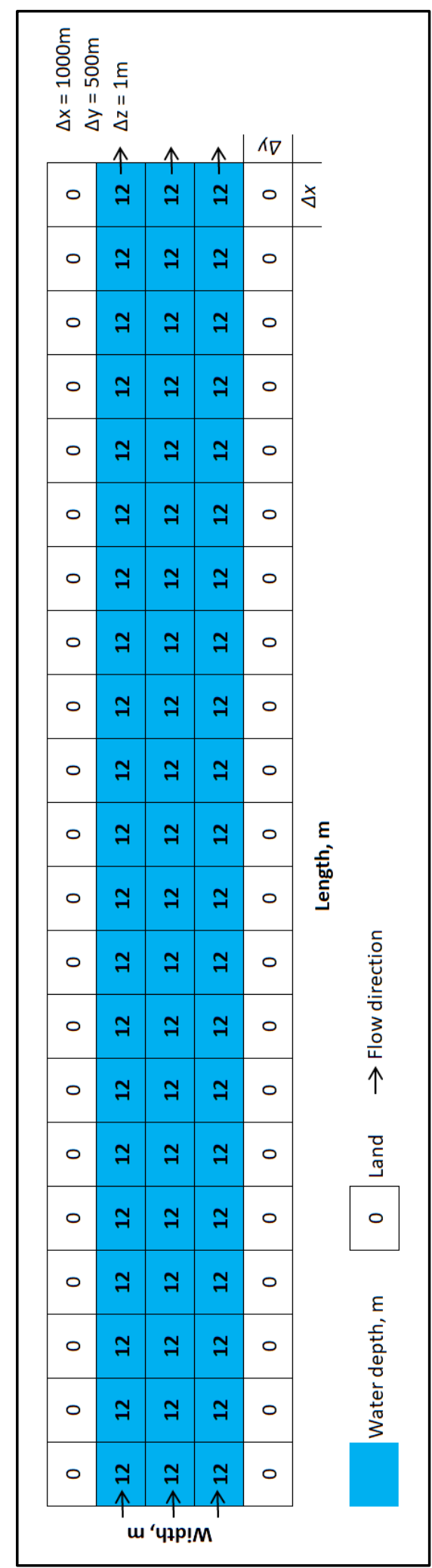

Figure 3-34. Test 4 rectangular physical domain and the input bathymetry 


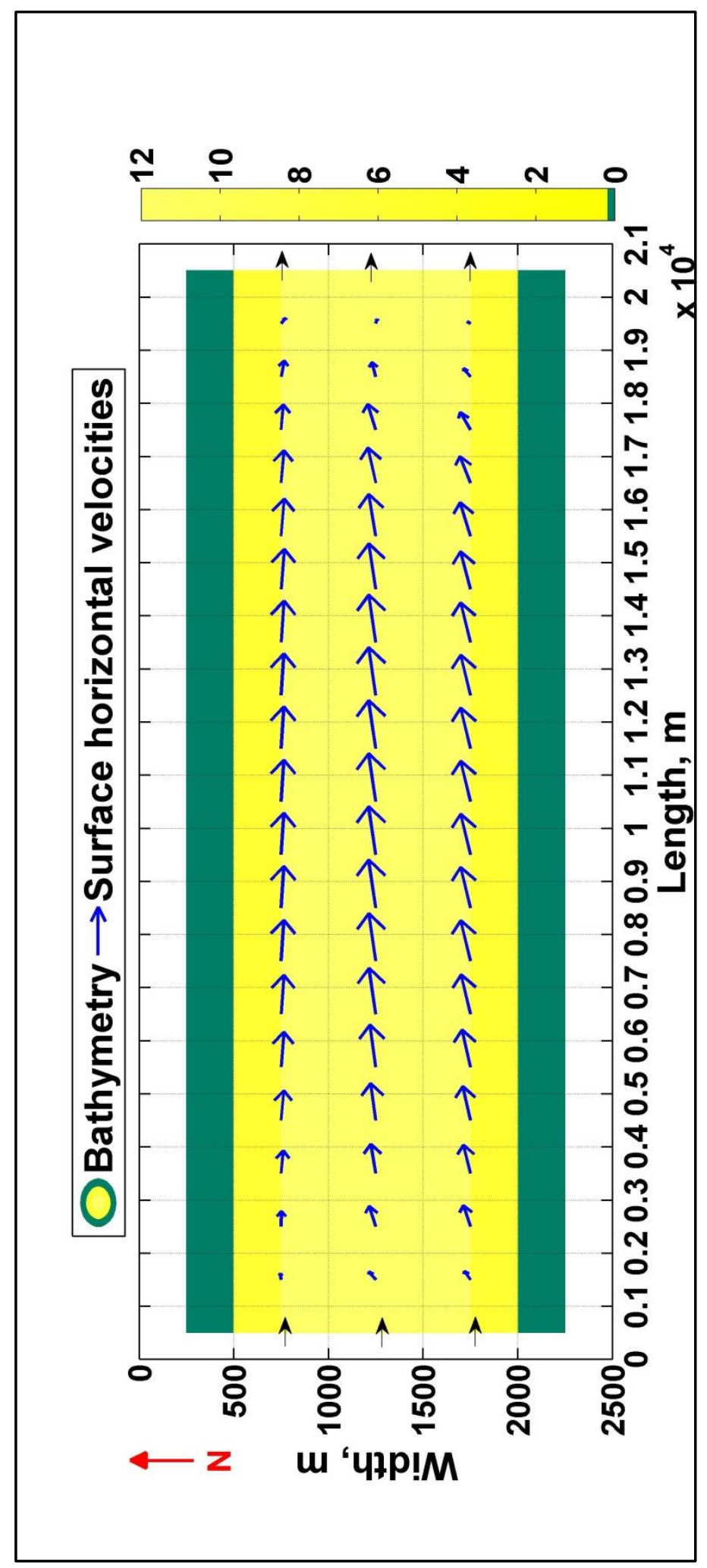

Figure 3-35. Test 4 surface velocity field for the rectangular domain at Julian day 100 


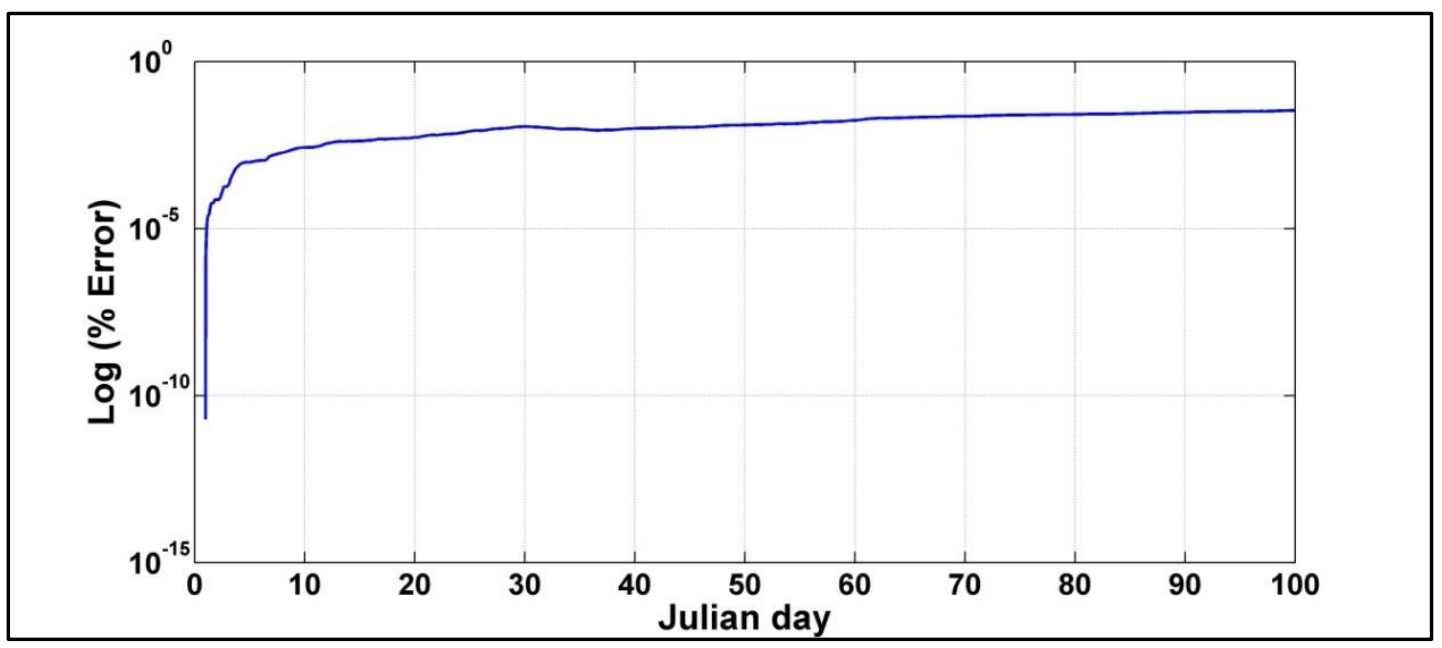

Figure 3-36. Test 4 volume balance for the rectangular domain using $\theta=1$

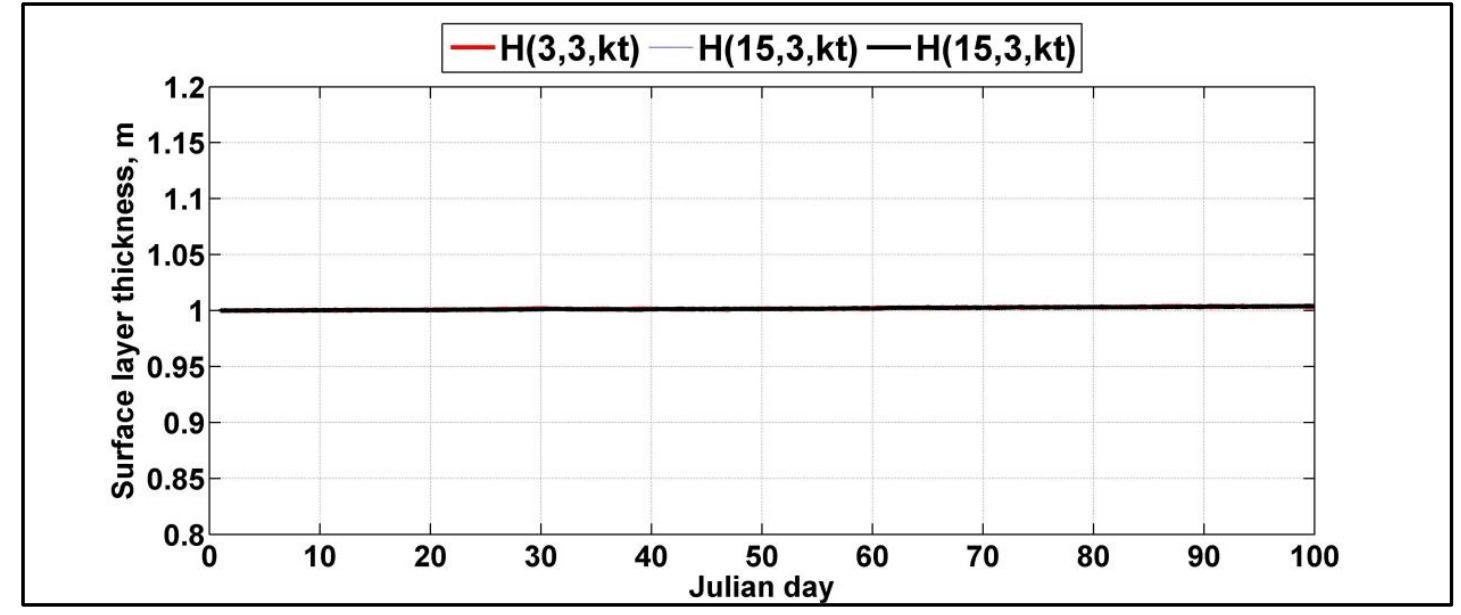

Figure 3-37. Test 4 surface layer thickness over time for the rectangular domain using $\theta=1$

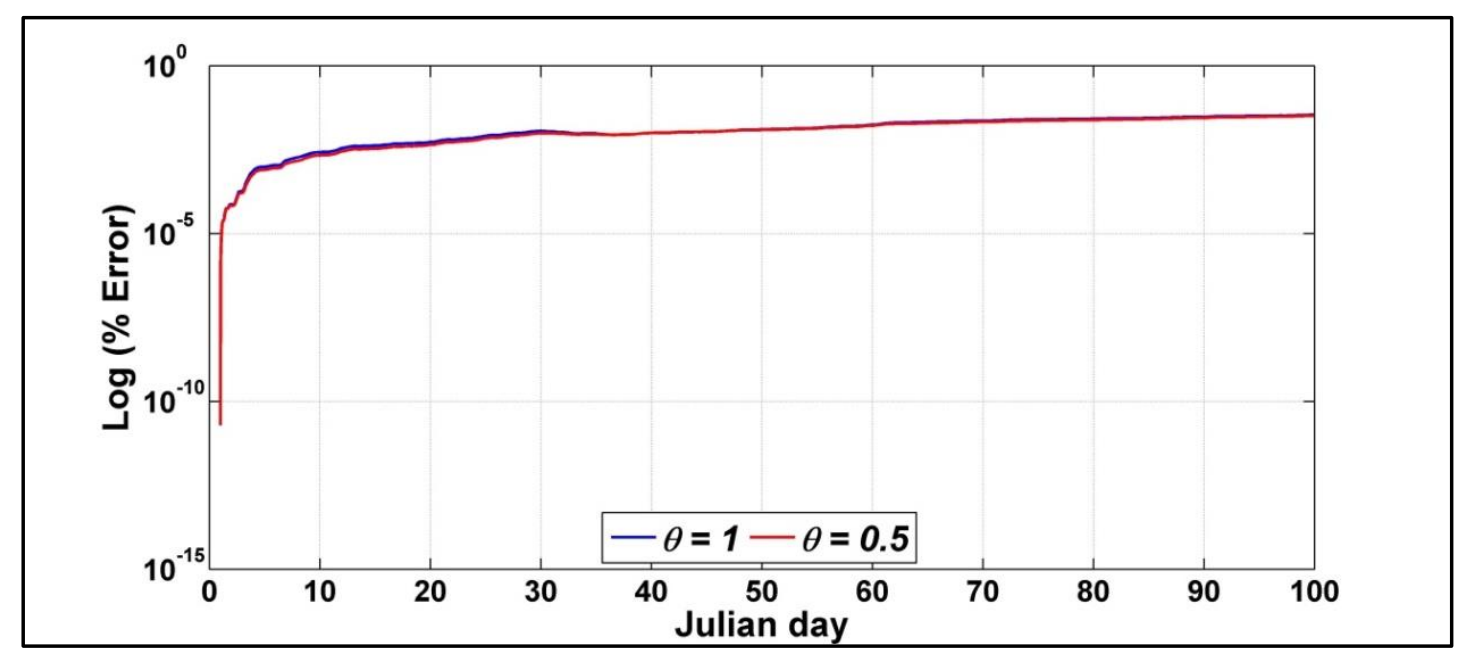

Figure 3-38. Test 4 the volume balance for the rectangular domain using $\theta=1$ and $\theta=0.5$ 


\subsection{Test 5: Model Sensitivity to the Bottom Resistance}

Generally, the bottom shear stress, which is a result of the bed resistance forces acting in the opposite direction to the flow, are inversely related to the Chezy coefficient as described in the quadratic drag law. As the Chezy coefficient becomes lower, the fluid acceleration or the flow velocity decreases, assuming all other parameters constant, because the bottom shear stresses become higher.

In order to show the influence of the bed resistance on the water level, a sensitivity test was performed using the rectangular physical domain of test 4 . Excluding the wind effect and surface heat exchange, two values of Chezy coefficient were examined ( $C=20$ and 25) with a constant flow rate of $2600 \mathrm{~m}^{3} / \mathrm{sec}$ at the left boundary. At the right boundary, the horizontal velocity was calculated using Chezy formula $(u=C \sqrt{ }(R S)$. After running the code using $\theta=0.55$, the influence of Chezy coefficient on the water level along the $x$ direction at a Julian day of 1.5 was as shown in Figure 3-39. The associated vertical velocity profiles at the location $(i=15$ and $j=3)$ were plotted as shown in Figure 3-40. Thus, Chezy coefficient is very important and this property is a primary calibration tool for water level in rivers and estuaries. 


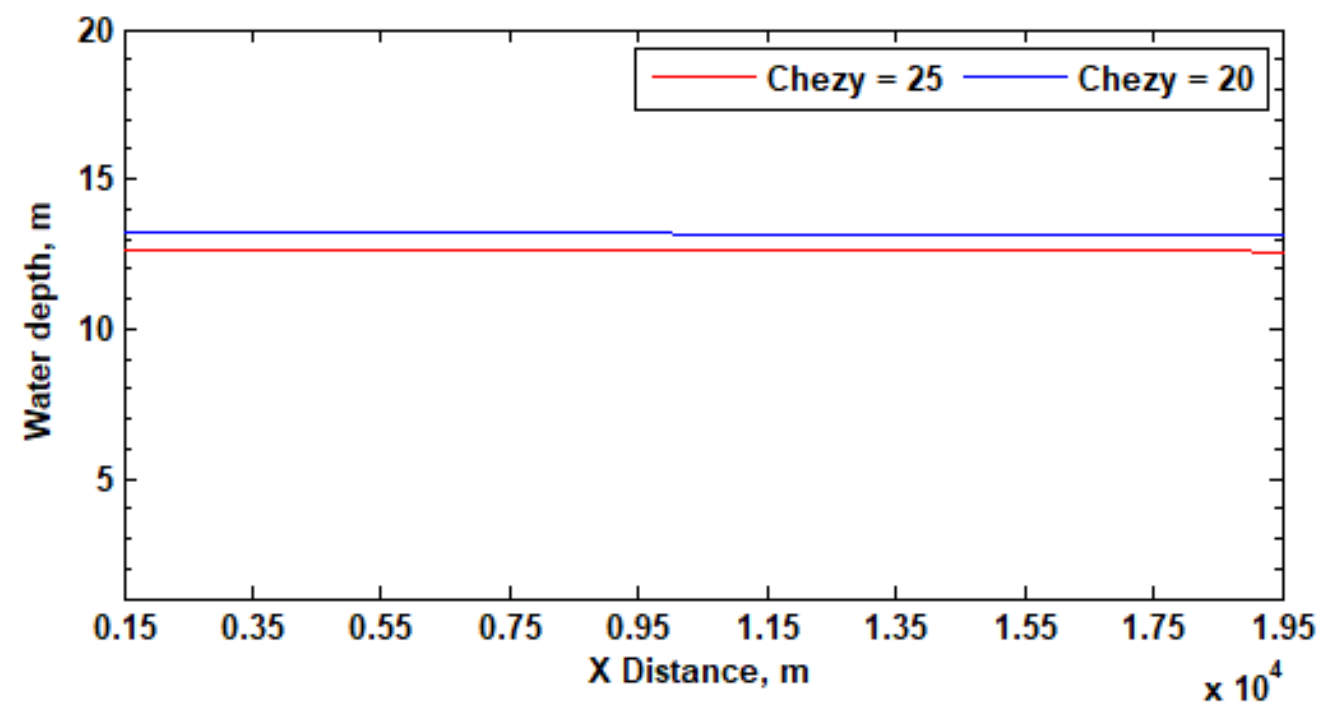

Figure 3-39. Test 5 water level sensitivity to the bottom resistance

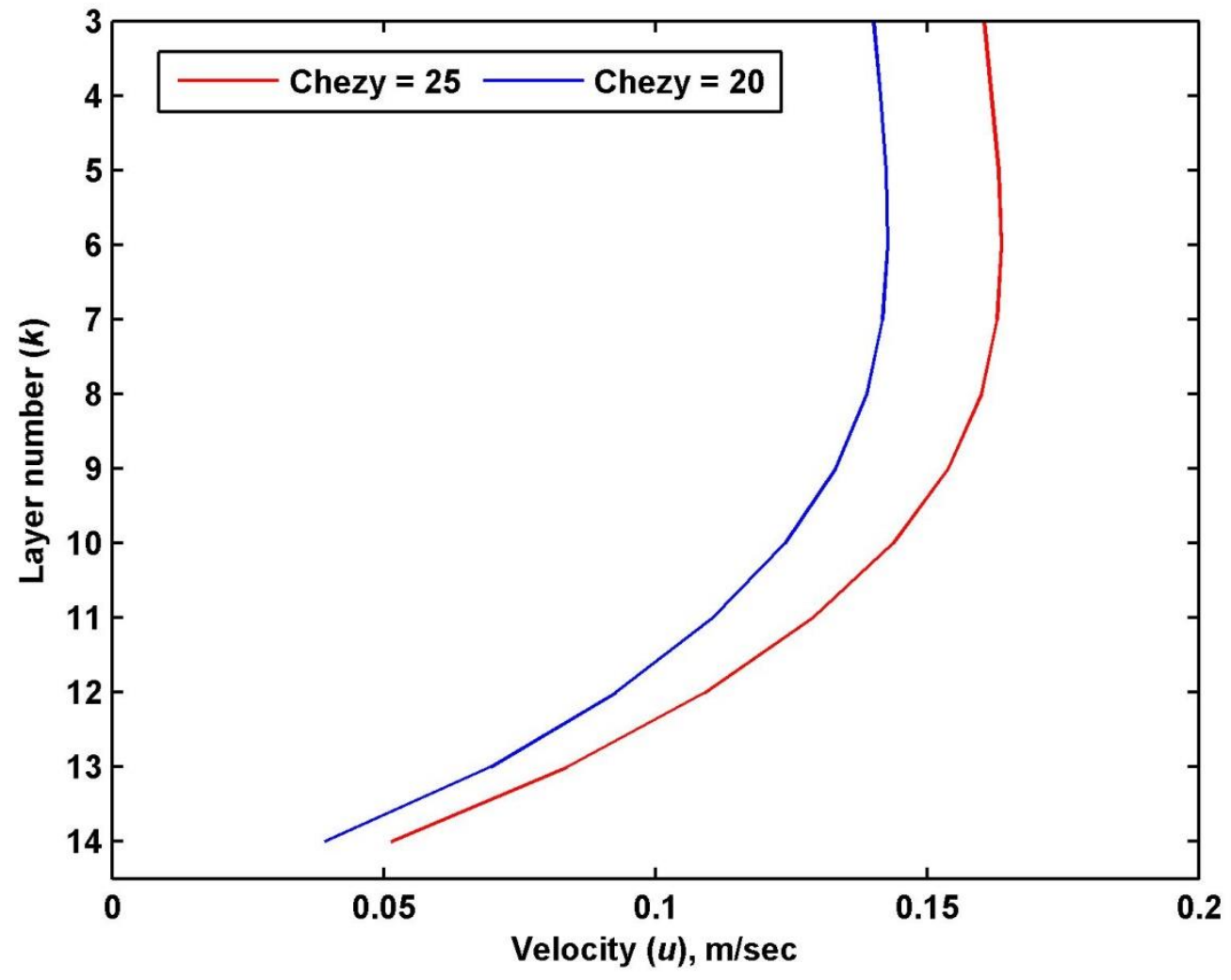

Figure 3-40 Test 5 vertical velocity profile sensitivity to the bottom resistance 


\subsection{Test 6: Model Sensitivity to the Wind Induced Flow}

Wind at the surface works in an opposite direction to the bottom shear stresses if the wind and flow direction are the same. The water surface layer is accelerated in the direction of wind. As the wind speed increases, the related surface shear stress also increases.

Therefore, we expect the amount of water that is moving through the domain due to the high wind to be higher if wind was in the same direction of the main flow (It will be lower if wind had an opposite direction to the main flow). Thus, wind speed and direction affect the water surface waves and the amount of transported water.

To correct the wind speed from a measurement location to the surface of the waterbody, a wind sheltering coefficient, WSC, was proposed that is multiplied by the measured wind speed (Cole \& Wells, 2017). The wind speed coefficient can be equal to 1 implying no correction to the wind speed or less than or greater than 1 . To study this effect, a sensitivity test was done using the same input data of test 4 and based on the irregular physical domain and $\theta=1$. Two values of WSC were used (WSC $=0.2$ and 1 ) and the surface wave at the point $(3,3, k t)$ was plotted as shown in Figure 3-41. Figure 3-42 shows the semi-log plot of the percent error with time related to each WSC. Higher wave amplitude of the water resulted from the higher value of WSC. Lower error in model volume resulted from the lower WSC. 


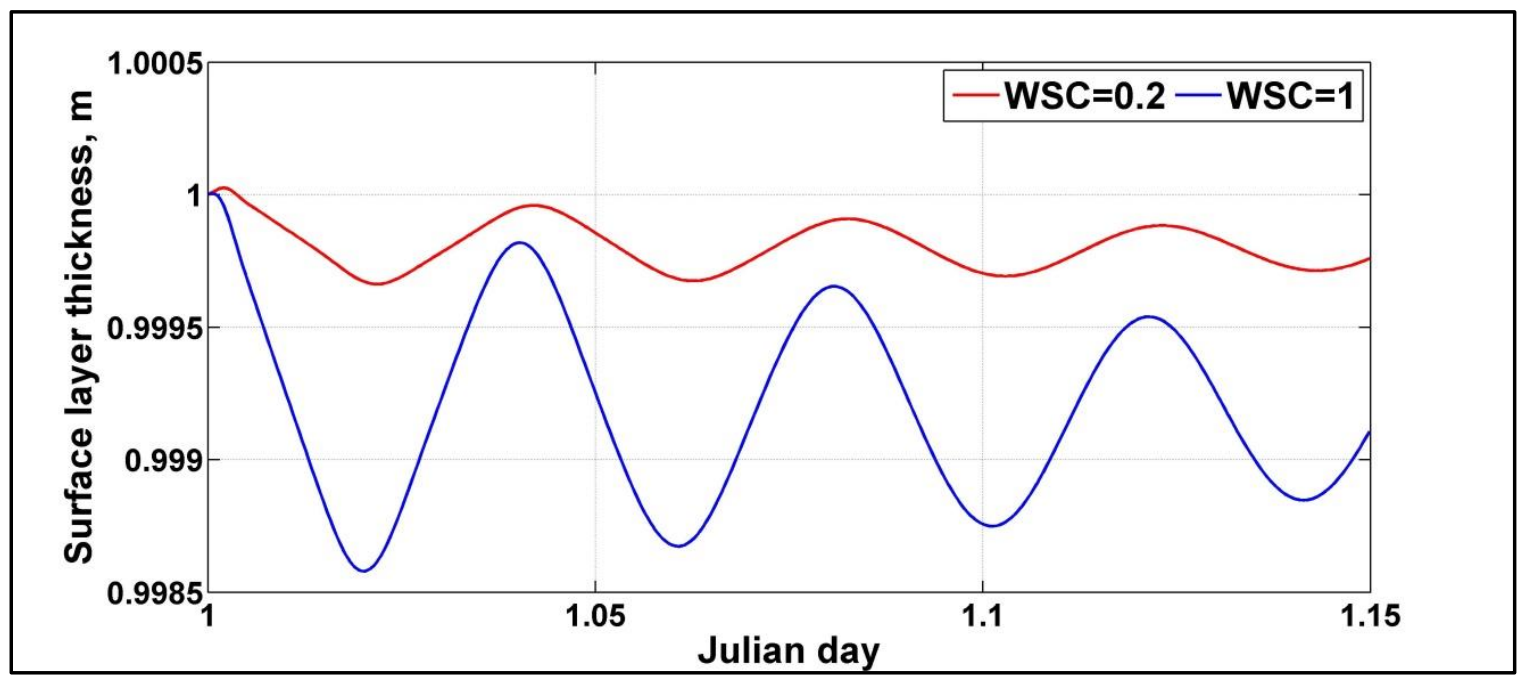

Figure 3-41. Test 6 model sensitivity to the wind induced flow near the left boundary $(i=3$, $j=3$, and $k=k t=3$ )

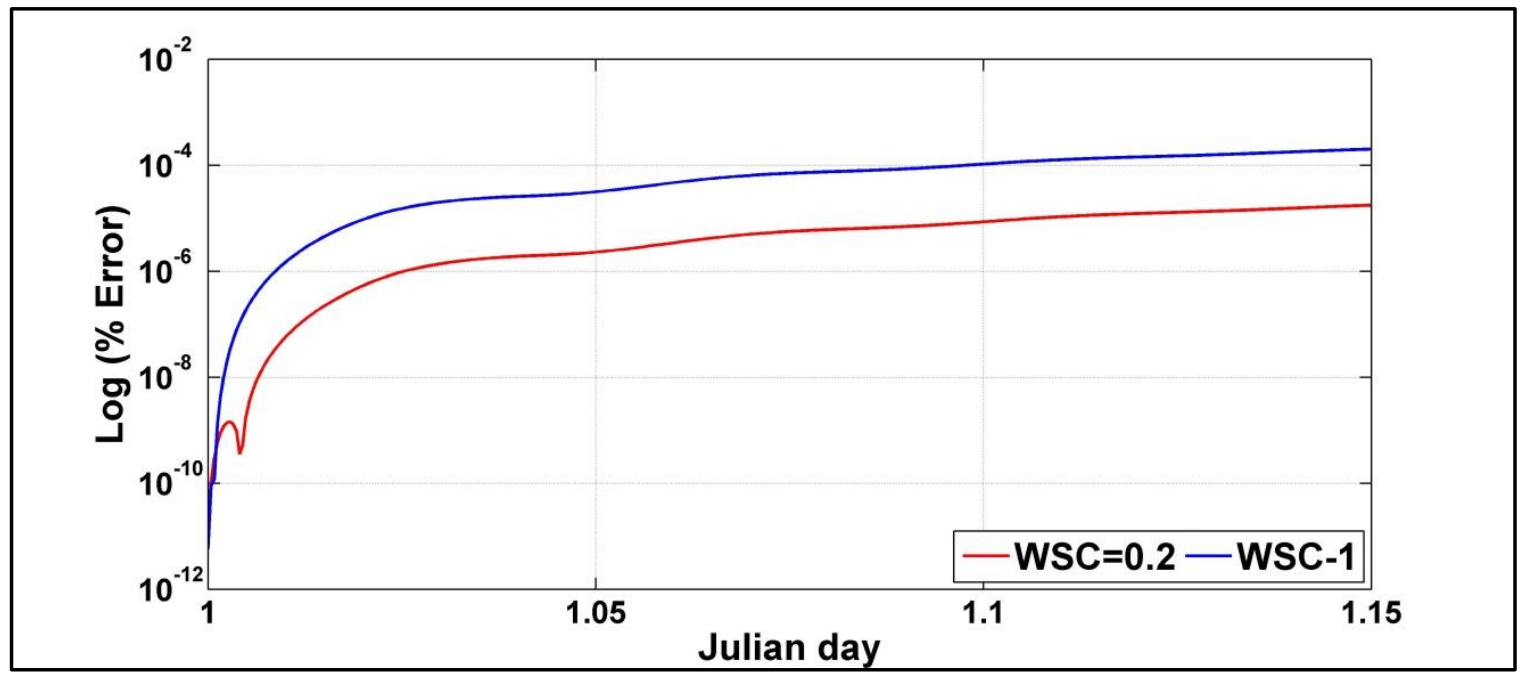

Figure 3-42. Test 6 volume balance 


\subsection{Test 7: Wetting/Drying Boundary Conditions}

In the code, at every time level the surface layer thickness is updated after finishing the water quality calculations (or temperature if water quality calculations are turned off). Because of the dynamic water depth, the model simulates the wetting/drying at the boundaries. Therefore, the 3D model employs the wetting/drying boundary conditions so that it allows the topography of the surrounding land to be inundated if the water level goes up or the wet grid cells to be dried if the water level goes down.

The governing equations are solved only within the wet domain boundaries. Therefore, the model distinguishes between the wet and dry cells by assigning zero water depth for the dry water columns. The water floods the area next to the wet boundaries when the surface layer water height (at $k=k t)$ reaches $85 \%$ the grid vertical thickness (i.e. $k=k t$ 1), and the boundary cells become dry when the surface layer water height (at $k=k t$ ) reaches $60 \%$ the grid vertical thickness (i.e. $k=k t+1$ ). The $85 \%$ and $60 \%$ were chosen based on the CE-QUAL-W2 algorithm for adding/subtracting layers.

For instance, Figure 3-43 shows an initial physical basin and the related bathymetry. This physical domain will be subjected to the step function inflow/outflow boundary condition in Figure 3-44. This setup allows the domain to be drained out and refilled during the simulation period. After running the model using the input meteorological data of test 4 , WSC of 1 , and $\theta$ of 0.55 , the basin bathymetry varied during the simulation period as shown in Figure 3-45 to Figure 3-52 (drying conditions followed by wetting conditions). 


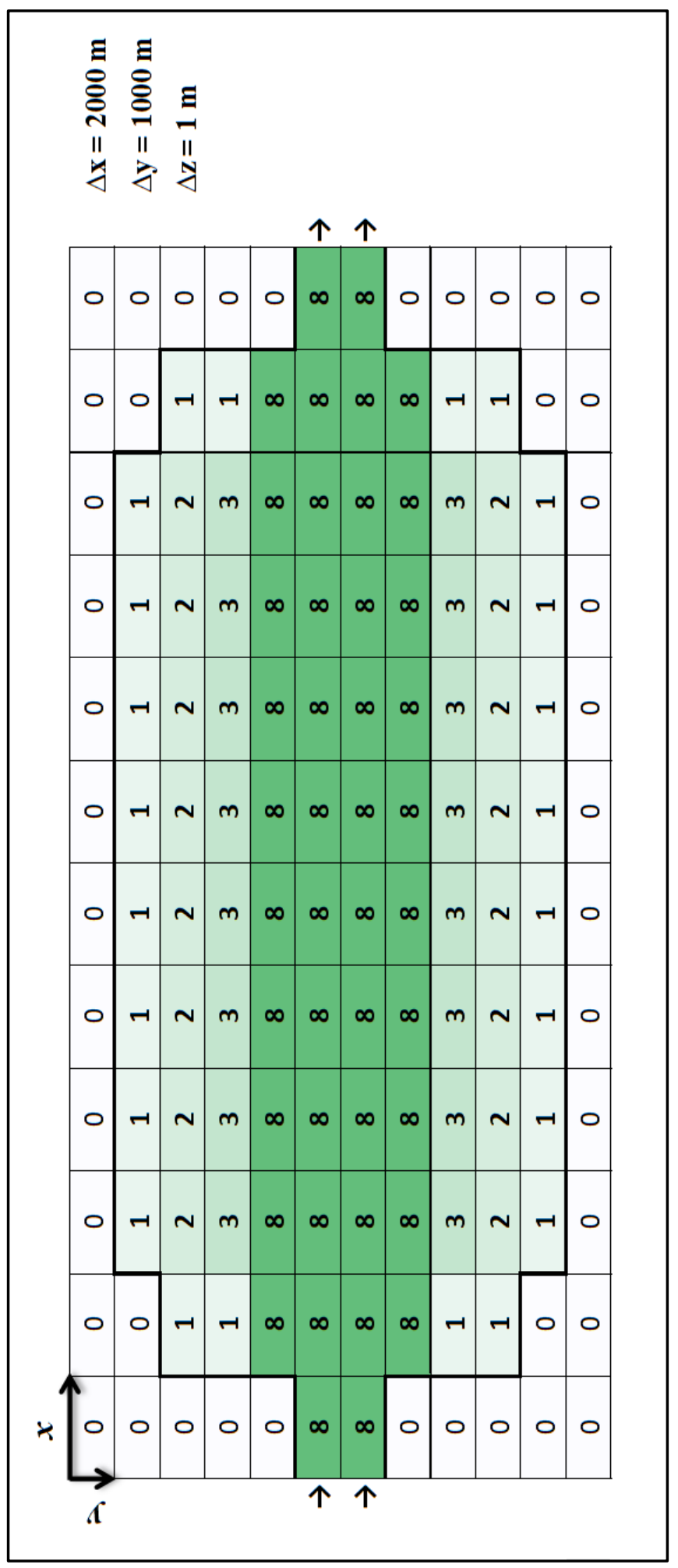

Figure 3-43. An initial physical domain bathymetry without the step function inflow/outflow boundary conditions 


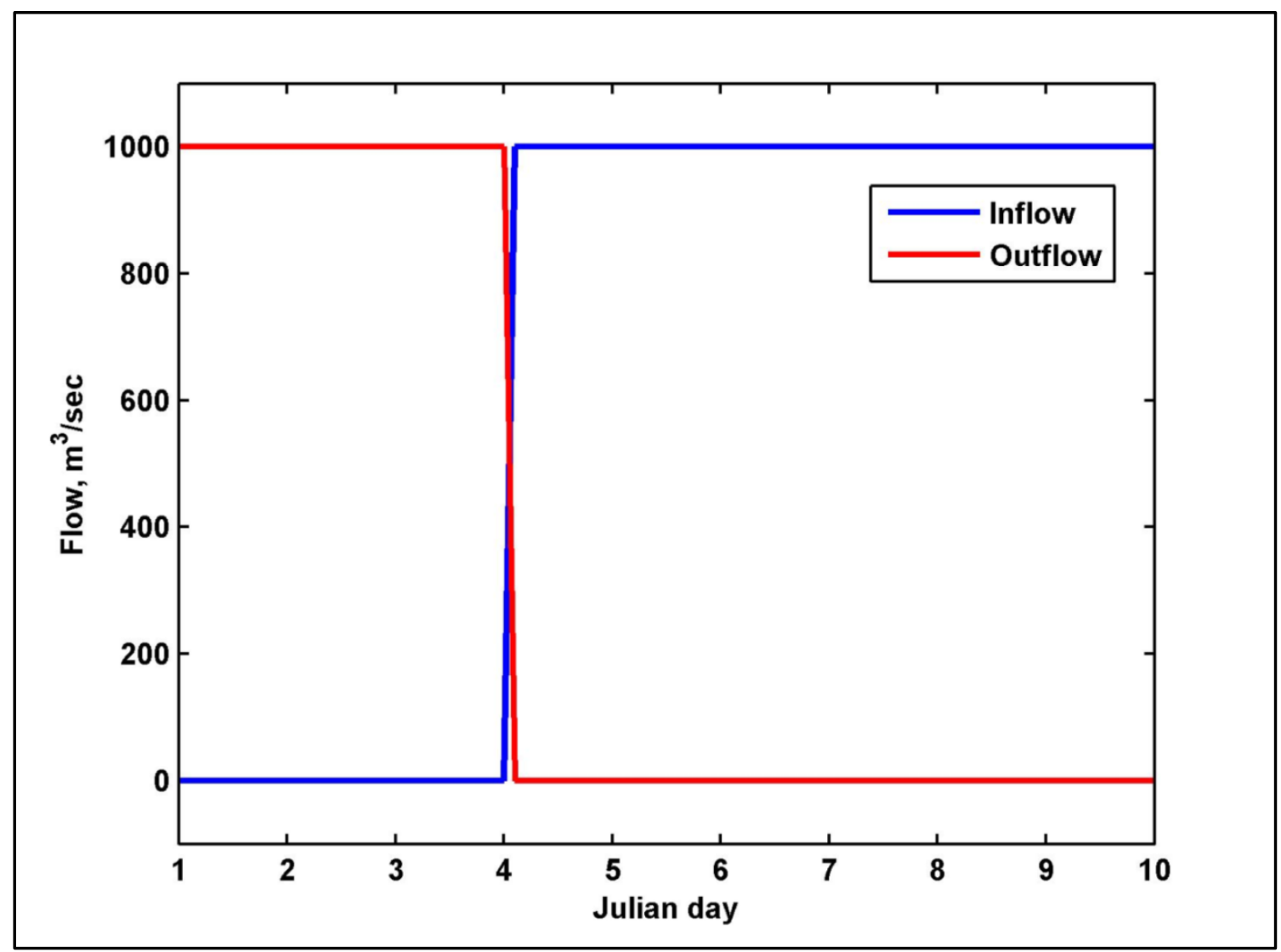

Figure 3-44. The step function inflow/outflow boundary conditions during the simulation period 


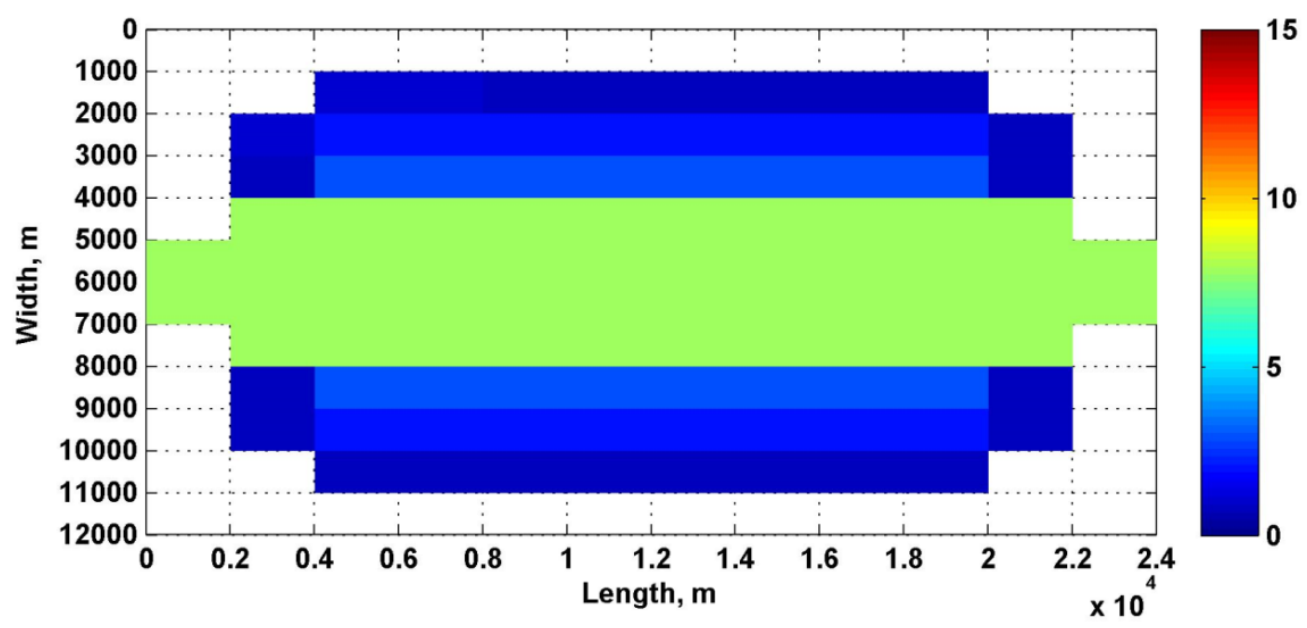

Figure 3-45. Drying conditions, Julian day $=1.15$

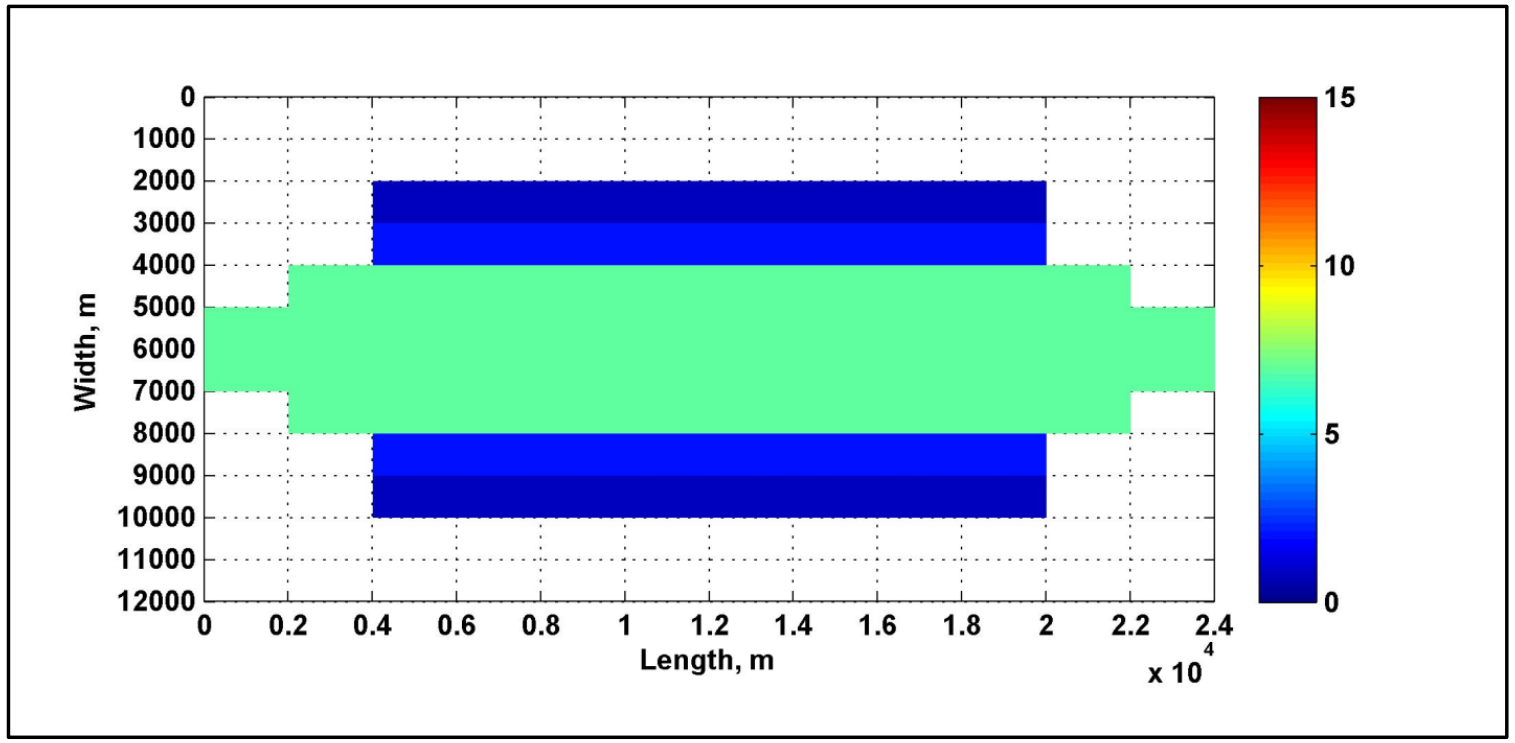

Figure 3-46. Drying conditions, Julian day $=3.15$ 


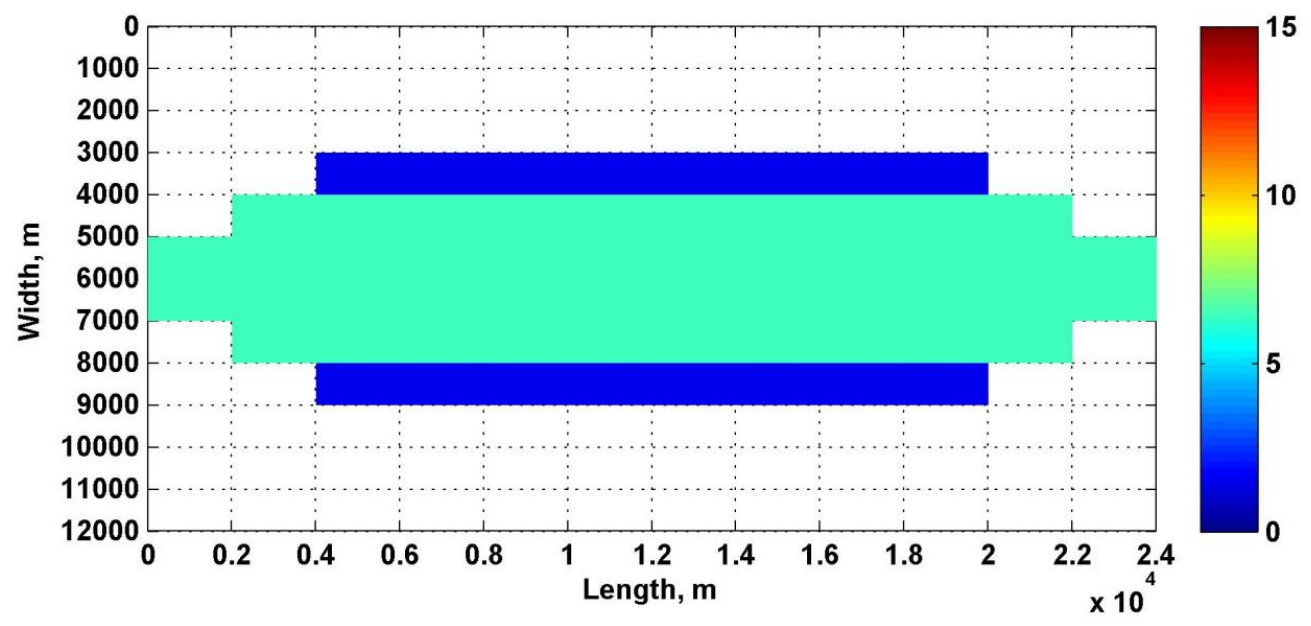

Figure 3-47. Drying conditions, Julian day $=4.15$

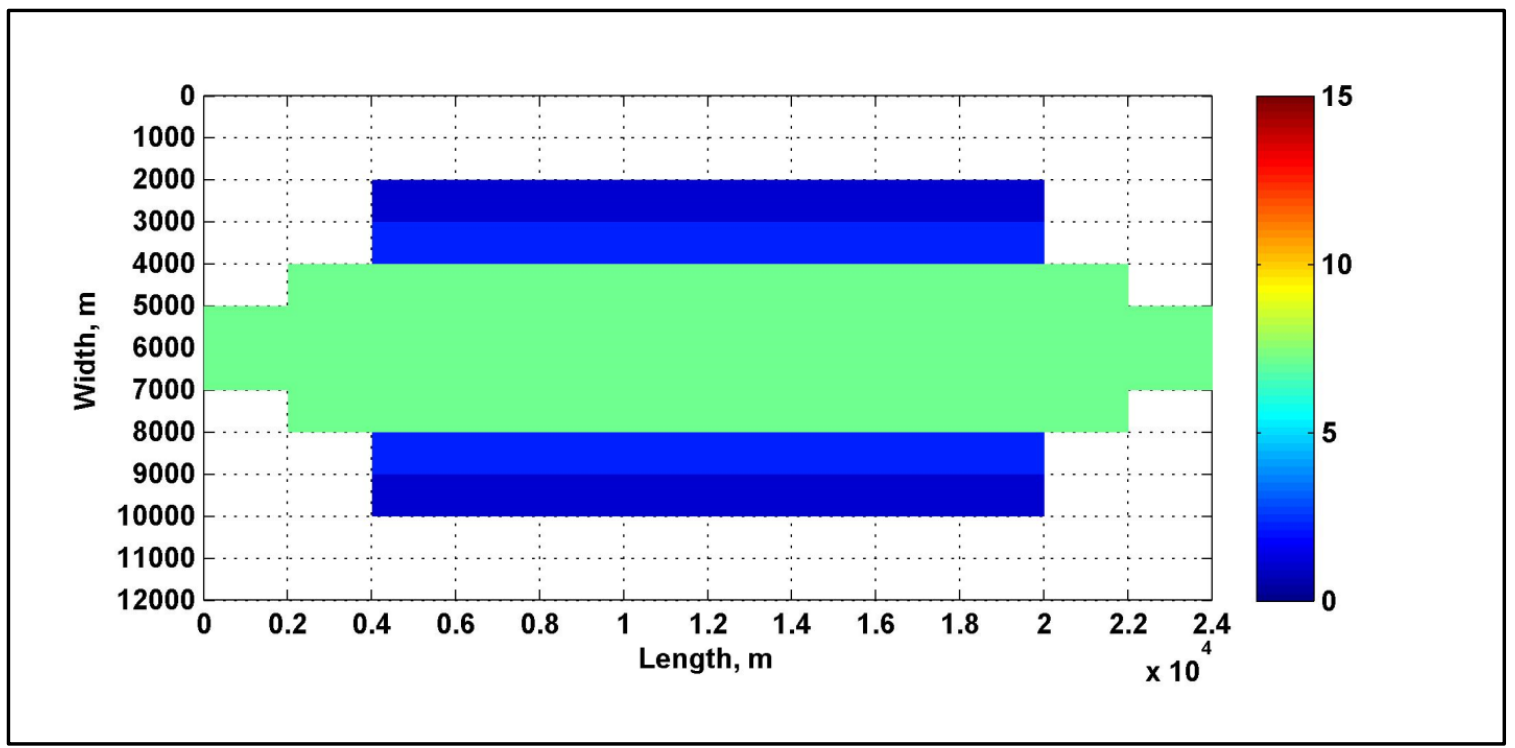

Figure 3-48. Wetting conditions, Julian day $=5.15$ 


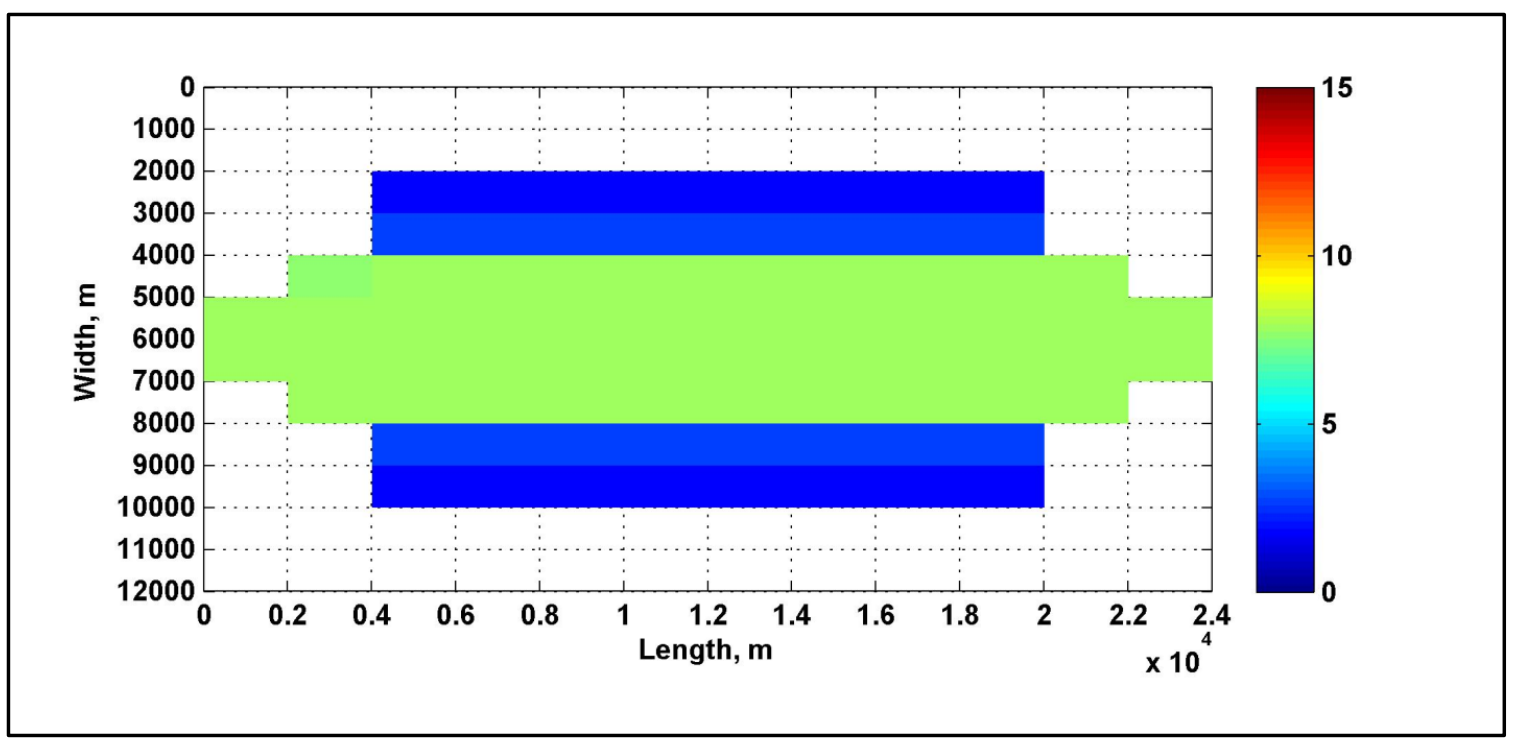

Figure 3-49. Wetting conditions, Julian day $=6.15$

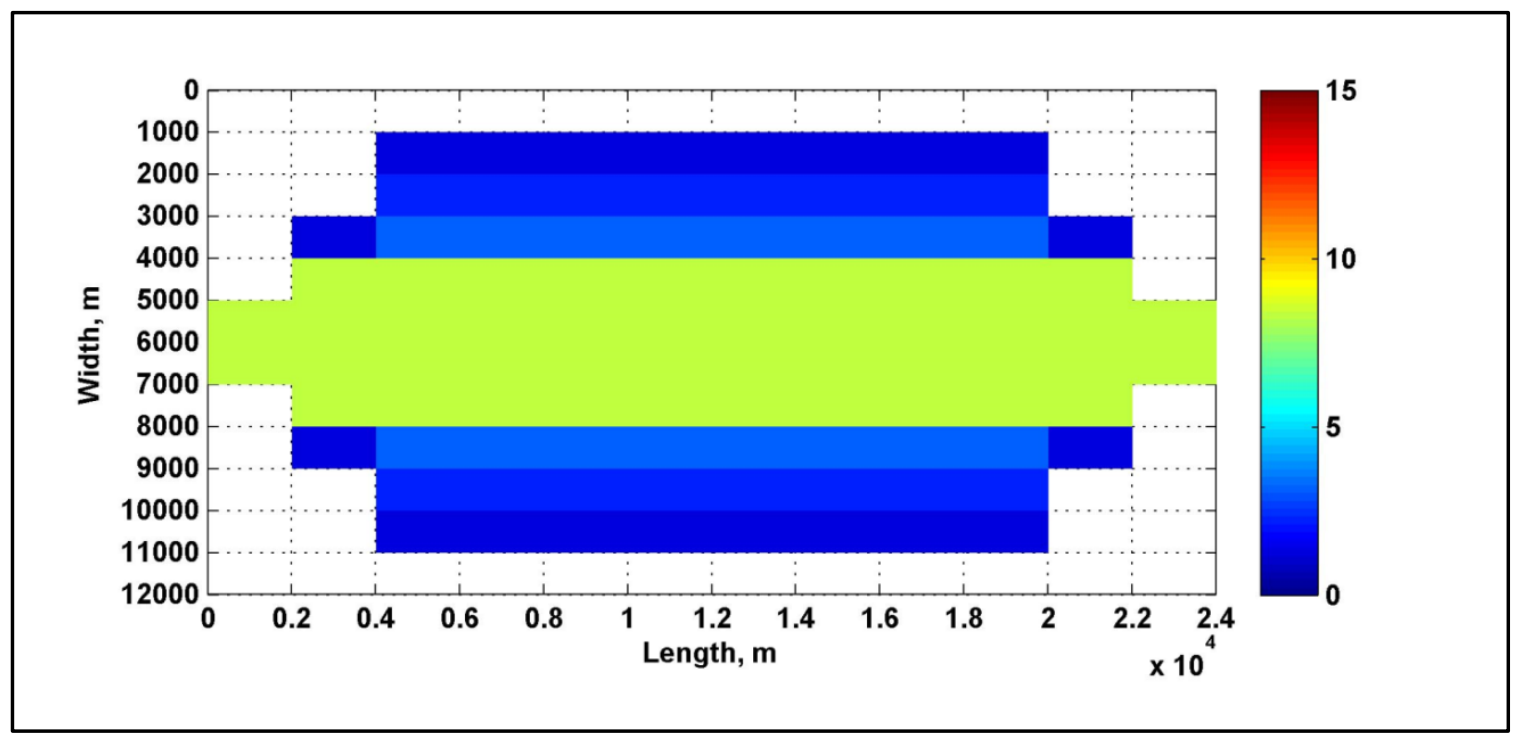

Figure 3-50. Wetting conditions, Julian day $=7.15$ 


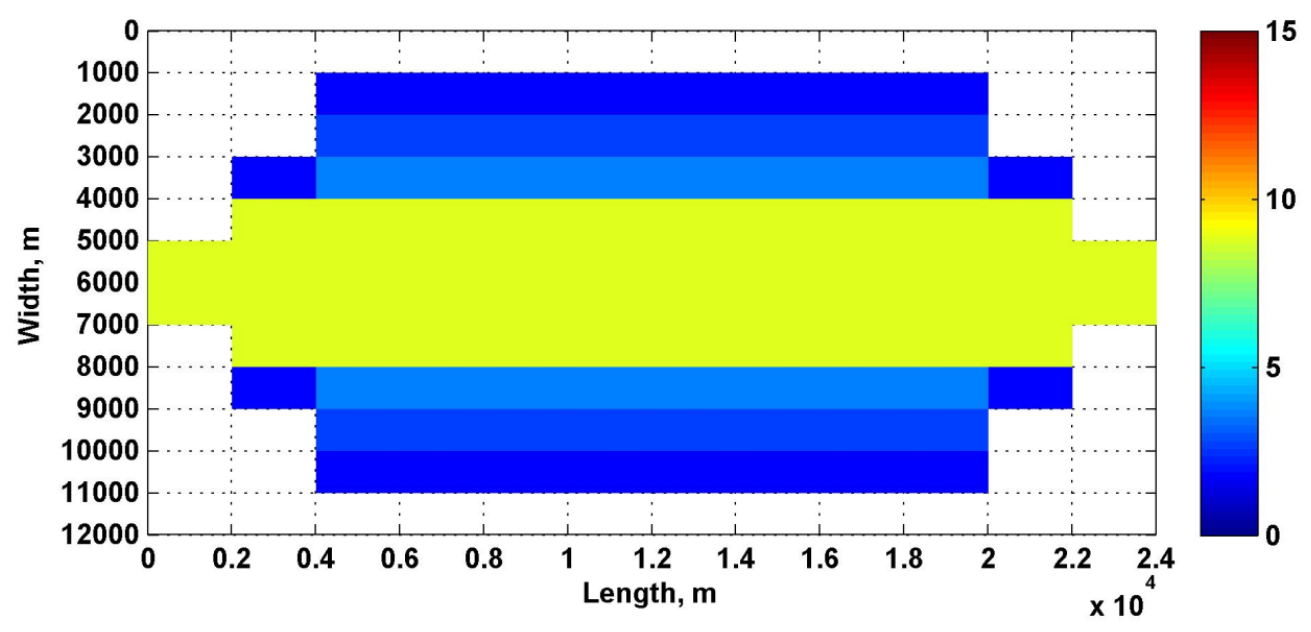

Figure 3-51. Wetting conditions, Julian day $=8.15$

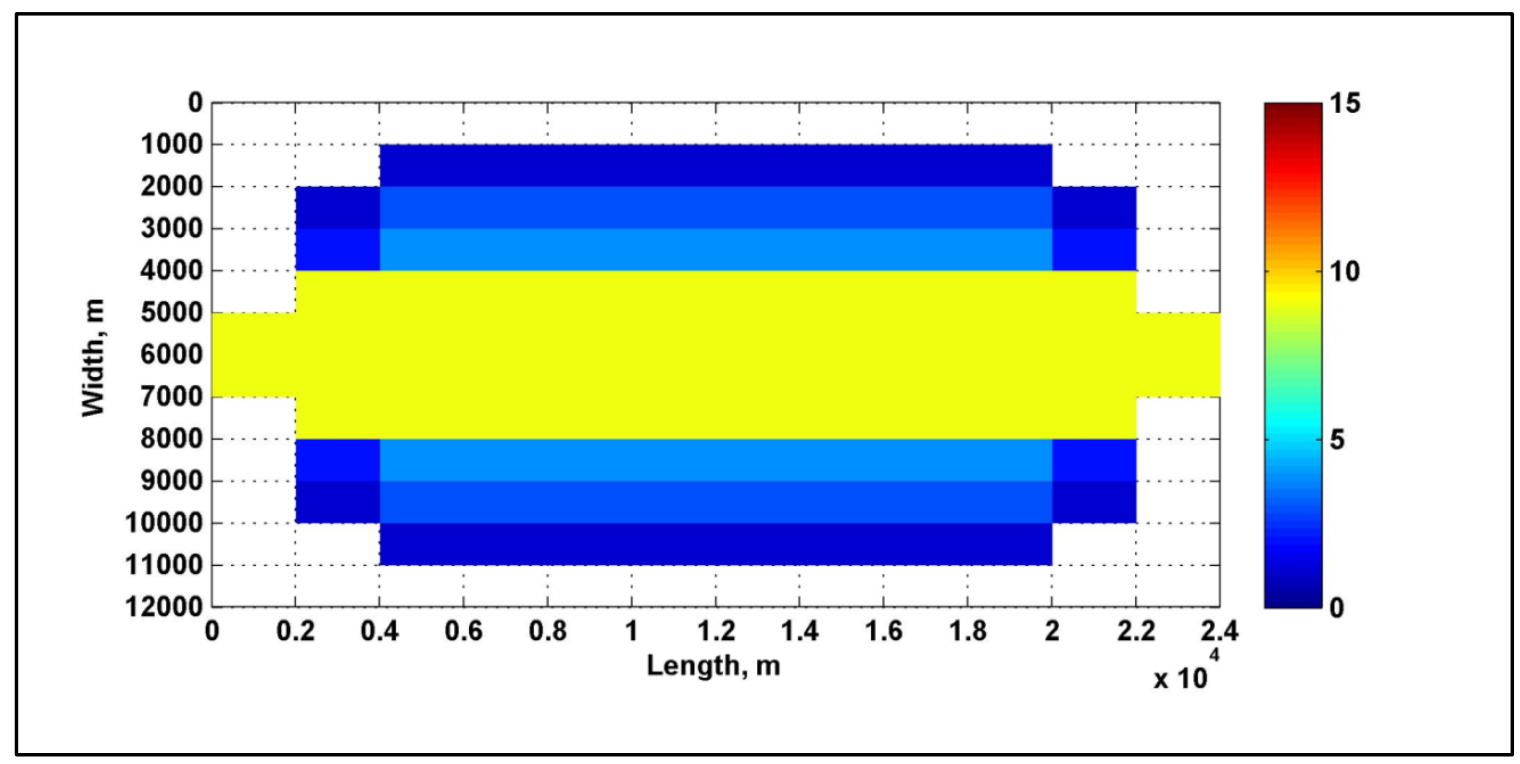

Figure 3-52. Wetting conditions, Julian day = 10.15 


\section{CHAPTER 4. RESULTS AND DISCUSSION: FIELD CASE STUDIES}

After developing and analytically verifying the numerical scheme of the threedimensional model, the model was validated by field case studies in three reservoirs in the USA. The model was validated by comparing model predictions of water levels, velocity, dissolved oxygen and vertical temperature profiles with field data. Through these real applications, we explored how well the model agreed with measured field data.

The model results of each field case study were discussed separately. In the first application, the study was focused on the importance of the higher-order schemes compared to the first-order UPWIND scheme for the advective transport equation. The model predictions of temperature were determined by using the UPWIND, QUICK, and QUICKEST schemes and compared with field data. In the second case study, the QUICKEST and ULTIMATE QUICKEST schemes were used to simulate a lake system, and a comparison was performed between the present 3D model and the 2D CE-QUALW2. Since the 3D model was build based on the 2D CE-QUAL-W2 numerical scheme, differences between the two models were evaluated. In addition, the feedback between the hydrodynamics and water quality was clarified by simulating the total suspended solids as a water quality constituent. Finally, a case study was done to show the model predictions of temperature and dissolved oxygen. In this application, dynamic vertical temperature profiles covered the entire simulation period through stratification and nonstratification conditions. 


\subsection{Case Study 1: Lake Chaplain}

A 3D W3 hydrodynamic and temperature numerical model was developed and calibrated for Lake Chaplain, Washington, USA. The governing equations are the continuity equation, free surface equation, momentum equation, and conservation equation of transport. The model employs the semi-implicit finite difference scheme to solve the governing equations and higher order schemes (QUICK and QUICKEST) for mass and heat transport in contrast to the simpler but more diffusive first order UPWIND scheme. The surface heat exchange and turbulence structure were based on the CE-QUAL-W2 model. Comparisons in water surface levels, velocities, and temperature vertical profiles between model predictions and data were performed using different advective transport schemes.

\subsubsection{Study Area Overview}

Lake Chaplain is a reservoir located in Snohomish County, Washington State, US. The latitude and longitude coordinates of Lake Chaplain are 47.9592309, -121.8447615 and the lake surface is at an approximate elevation of $650 \mathrm{ft}(198 \mathrm{~m})$ above sea level. The Lake Chaplain location within the surrounding watershed is shown in Figure 4-1 and the surrounding terrain is as shown in Figure 4-2. The inflow to the lake is a diverted flow from Spada Lake. There are two withdrawal outflows from Lake Chaplain; one of them is a pipe withdrawal flow from the lake north end toward a paper mill, the other is a drinking water withdrawal flow through an outflow structure at the dam which is at the lake south end. Lake Chaplain was modeled using the new three-dimensional numerical model to simulate hydrodynamic and temperature distributions in the lake. The required 
input data, lake bathymetry, and data required for calibration were available in a technical report prepared by the Water Quality Research Group in Department of Civil and Environmental Engineering at Portland State University (Annear et al. 2008).

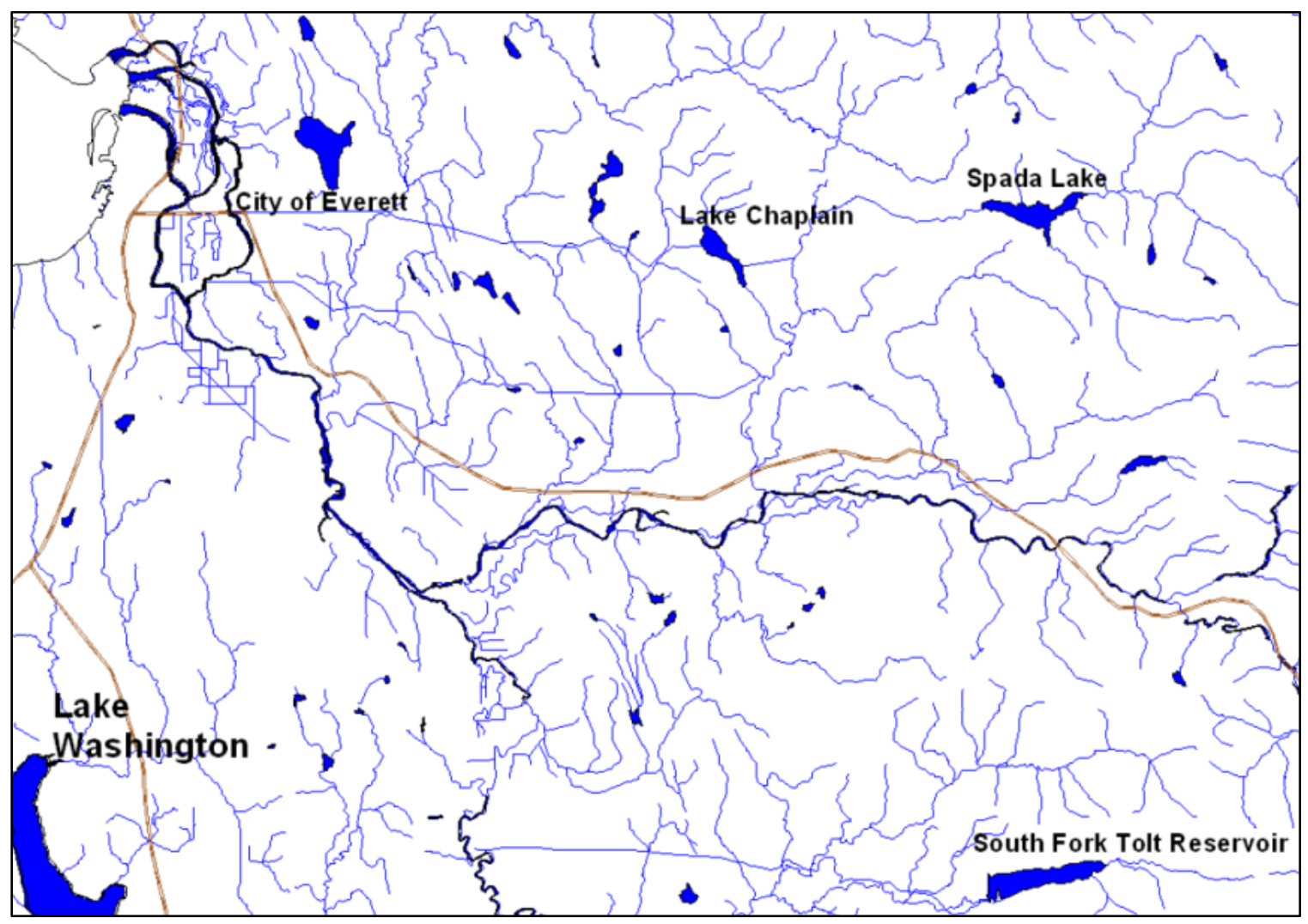

Figure 4-1. Lake Chaplain watershed (from Annear et al 2008) 


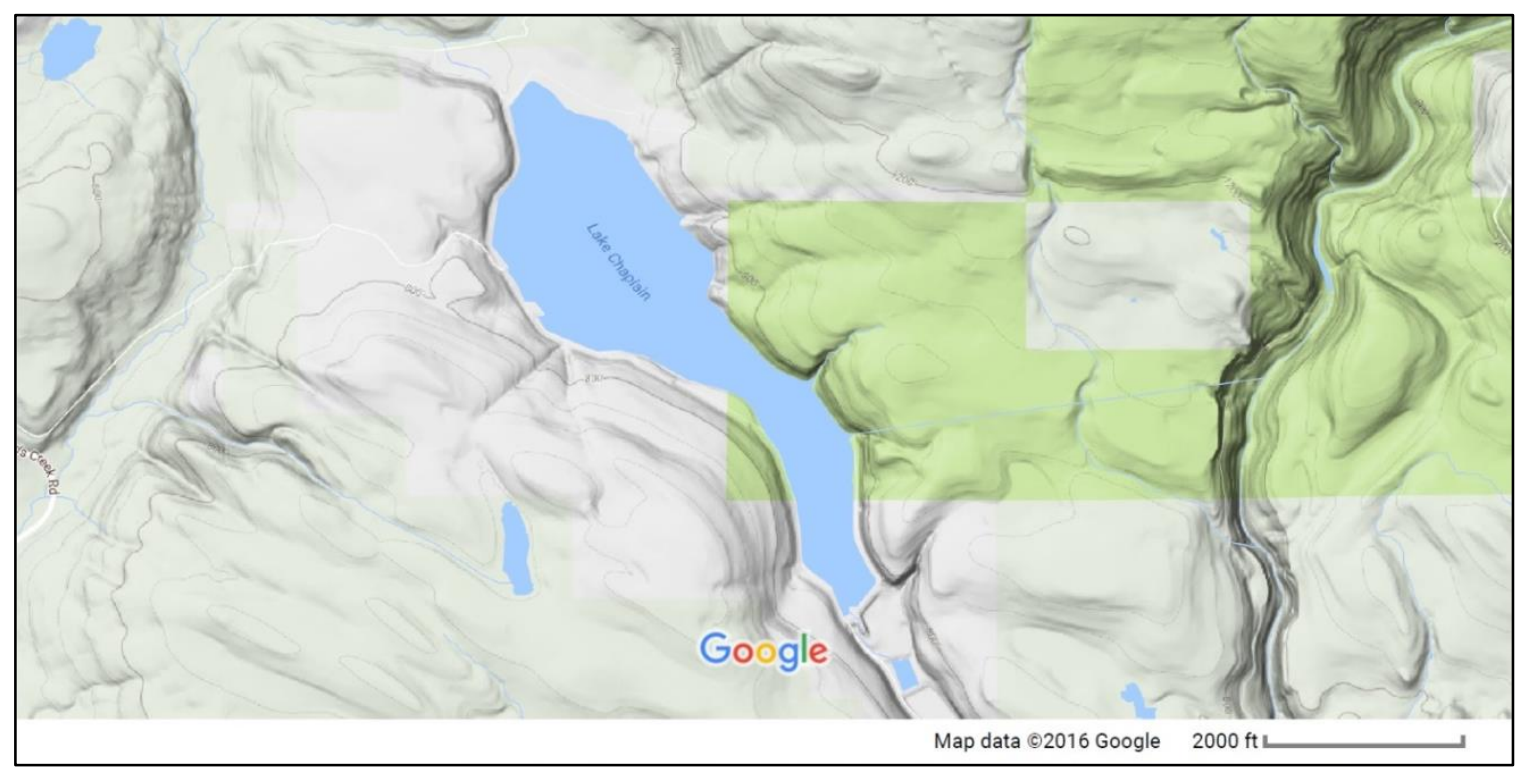

Figure 4-2. Lake Chaplain surrounding terrain (Google, 2016)

\subsubsection{Input Bathymetry and Model Grid Development:}

The surface contour map of Lake Chaplain was used to create the input bathymetry. Above the contour map, the physical domain of the lake was divided into computational grid cells as shown in Figure 4-3, which is a contour plot top view of the lake surface. The resolution of the computational grid cells was $\Delta x=220 \mathrm{~m}, \Delta y=110 \mathrm{~m}$, and $\Delta z=$ $1 \mathrm{~m}$. Figure 4-3 setup indicates that the angle that the grid makes with the northern direction (measured clockwise from the north) is 5.49 radians, which is required by the model for calculating wind shear stresses. Based on the available data (Aug10, 2007 Oct11, 2007), the initial water surface level was at an elevation of $195.73 \mathrm{~m}$. The above information was combined to create the input bathymetry file of the model. Figure 4-4 shows the model physical domain, input water depths, and boundary conditions location of the Lake Chaplain model. The initial water surface elevation was set to be at the top 
face of the surface computational cells in which $k=k t=3$. Therefore, the initial values of $\eta_{(x, y, t=0)}$ were set to zero.

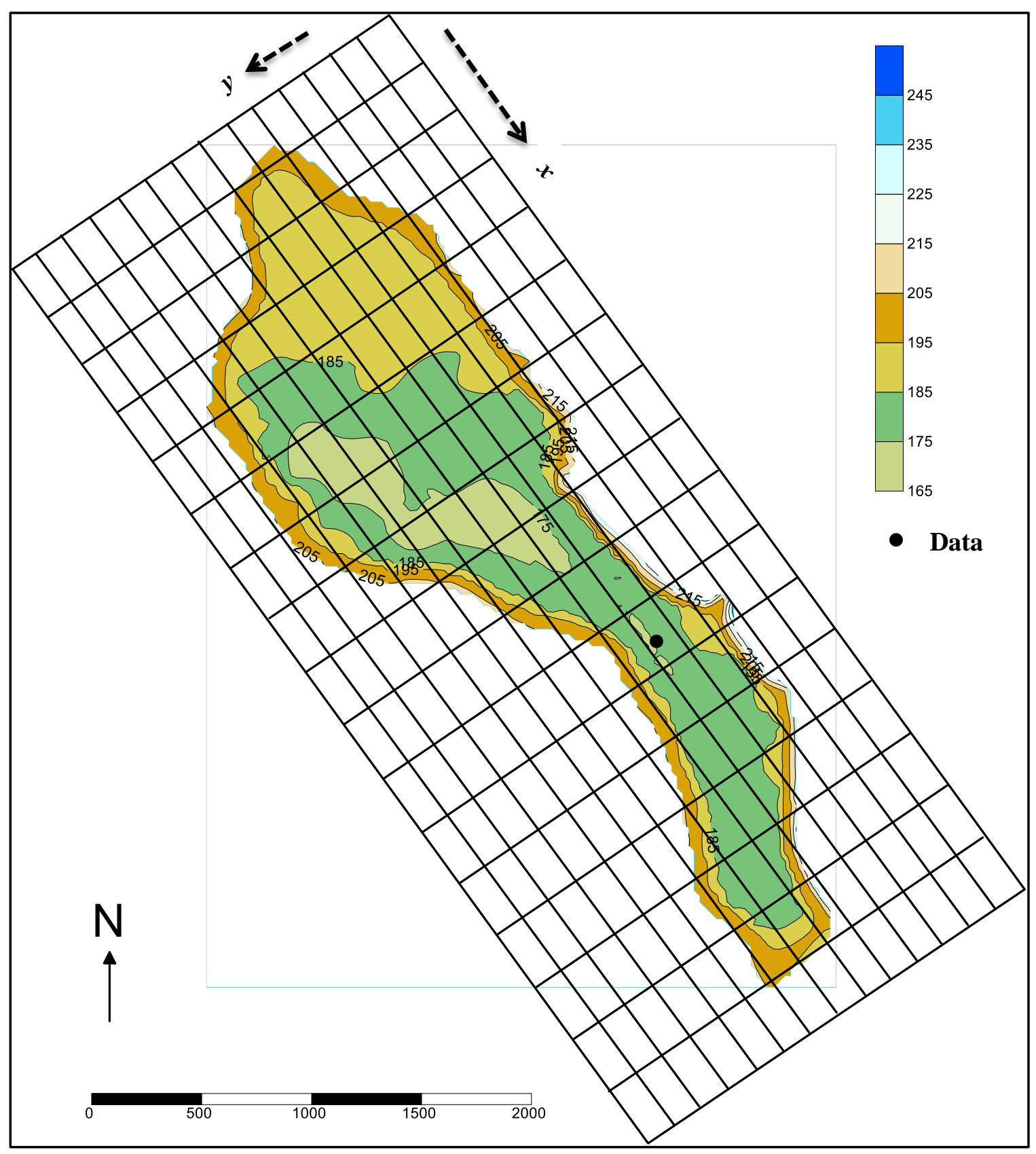

Figure 4-3. Top view of lake Chaplain model grid 


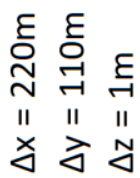

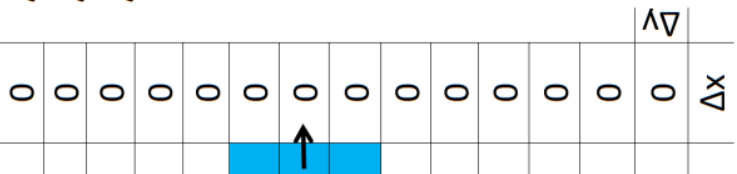

$$
\begin{aligned}
& 000000 \text { 의 } \\
& \text { - } 0000 \text { ㄴำ }
\end{aligned}
$$

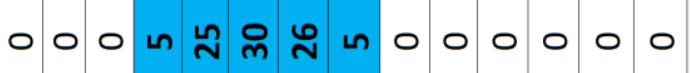

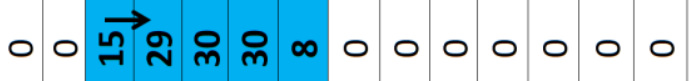

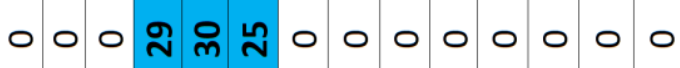

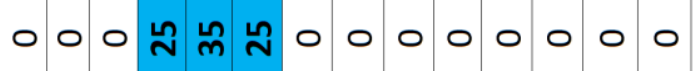

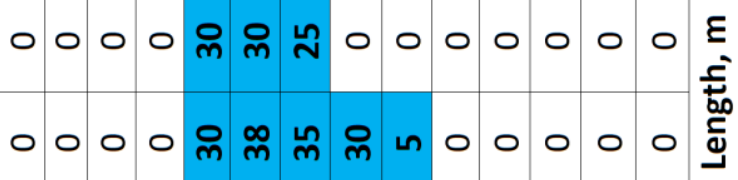

$$
\begin{aligned}
& \text { ○。 }
\end{aligned}
$$

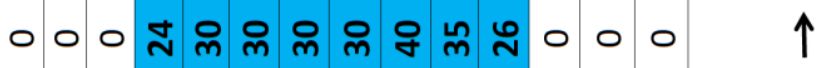

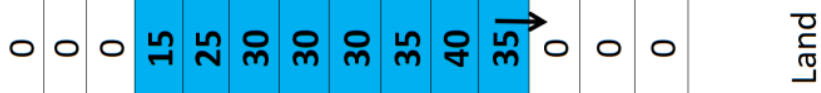

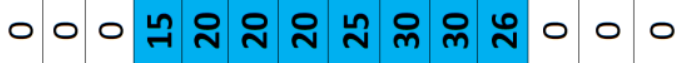

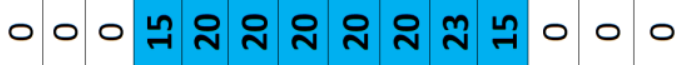

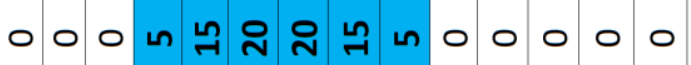

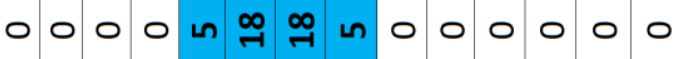

$$
\begin{aligned}
& 00000000000000000 \\
& \text { u 'чтр!м }
\end{aligned}
$$

Figure 4-4. The model physical domain of Lake Chaplain 


\subsubsection{Inflow/Outflow Boundary Conditions and Meteorological Data}

The inflow to the domain enters the main waterbody at a water depth of $15 \mathrm{~m}$. There is an outflow to a paper mill that is located at an elevation of $172 \mathrm{~m}$ at the model grid point $i=$ 6 and $j=11$, and there is another outflow to a water treatment plant located at an elevation of $184.8 \mathrm{~m}$ at grid point $i=16$ and $j=7$, the dam location. Figure $4-5$ to Figure 4-7 are the time series of the inflow and outflow that were used as boundary conditions to the model over the simulation period (Julian day: 222 - 284). In addition, the inflow temperature boundary condition varies over the simulation time (see Figure 4-8).

Within the lake domain, the initial temperature distribution was based on field data measurements at the dam. Figure 4-9 and Figure 4-10 show the initial vertical temperature profile and velocity distribution at the withdrawal locations. Every time step, the model distributes a new vertical velocity profile at the outflow locations depending on the vertical density variation by using the selective withdrawal theory (Imberger \& Fischer 1970). The 3D model adapted the theory implementation in the 2D CE-QUAL$\mathrm{W} 2$, and modified the $2 \mathrm{D}$ algorithm for the $3 \mathrm{D}$ case.

Wind magnitude and direction, air temperature, dew point, and cloud cover were necessary inputs for the water heat budget and surface heat exchange. Meteorological data sets and monitoring sites were described in Annear et al. (2008). Figure 4-11 and Figure 4-12 show the wind speed magnitude and direction at $2 \mathrm{~m}$ height, respectively. Figure 4-13 to Figure 4-15 show the time series of the air temperature, dew point, and 
cloud cover, respectively. These data were used in 3D Lake Chaplain model. The solar short wave radiation was calculated internally based on the algorithm of EPA (1971).

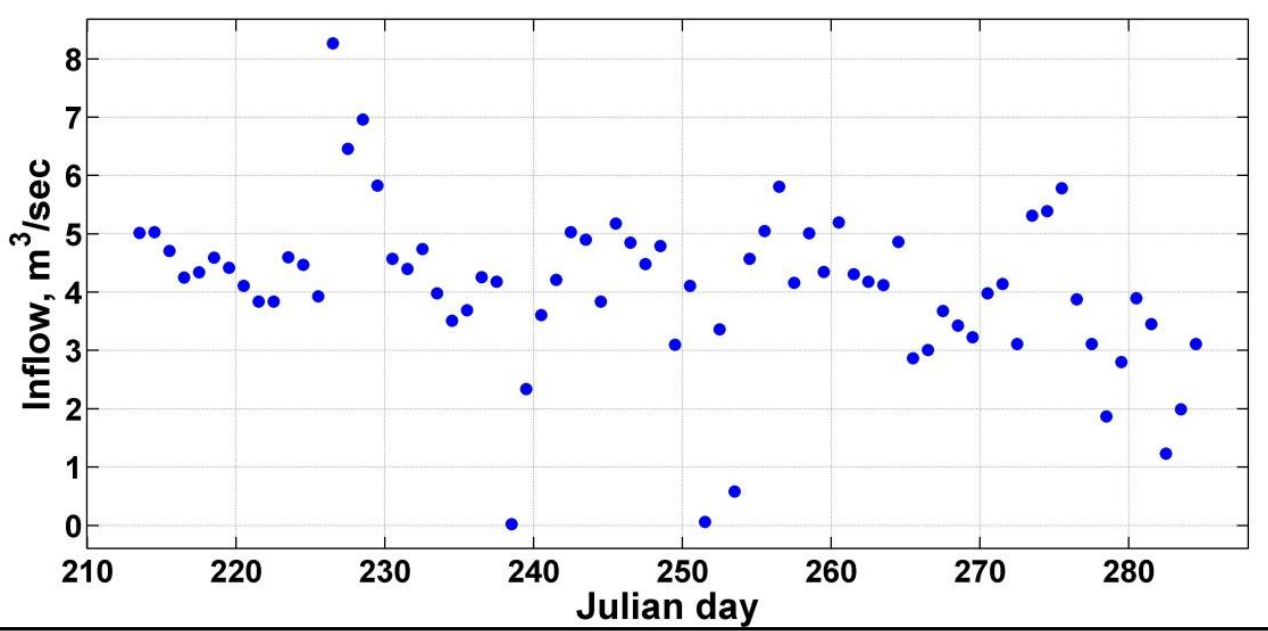

Figure 4-5. Lake Chaplain inflow

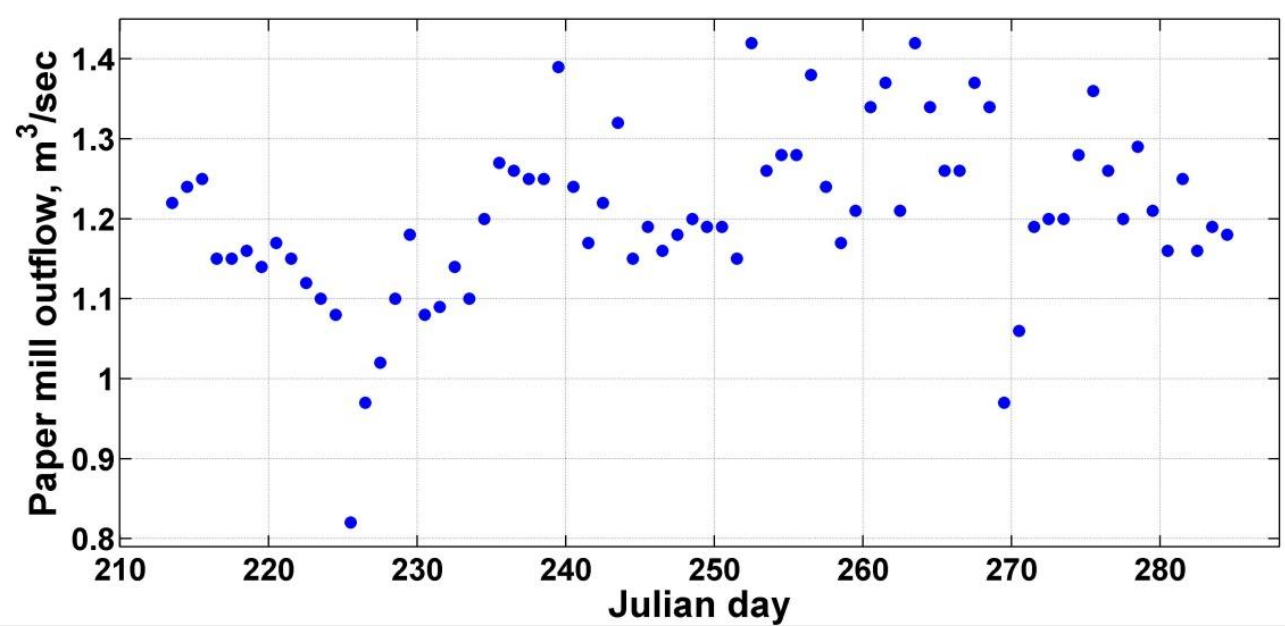

Figure 4-6. Lake Chaplain outflow to the paper mill 


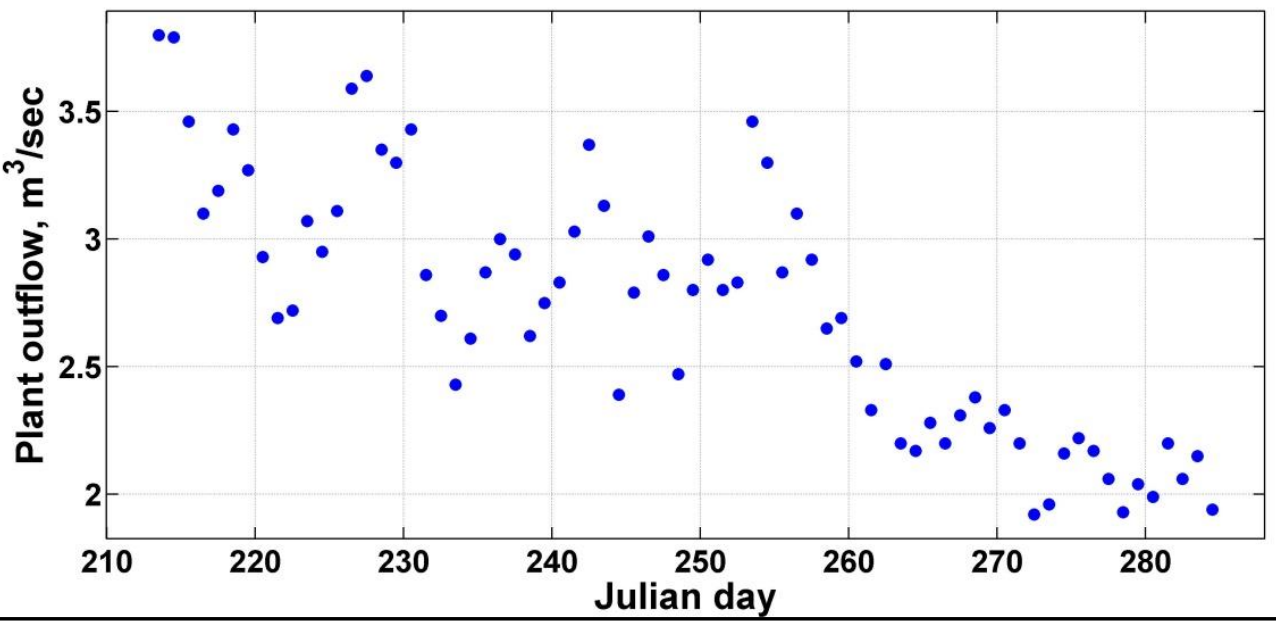

Figure 4-7. Lake Chaplain outflow to the water treatment plant

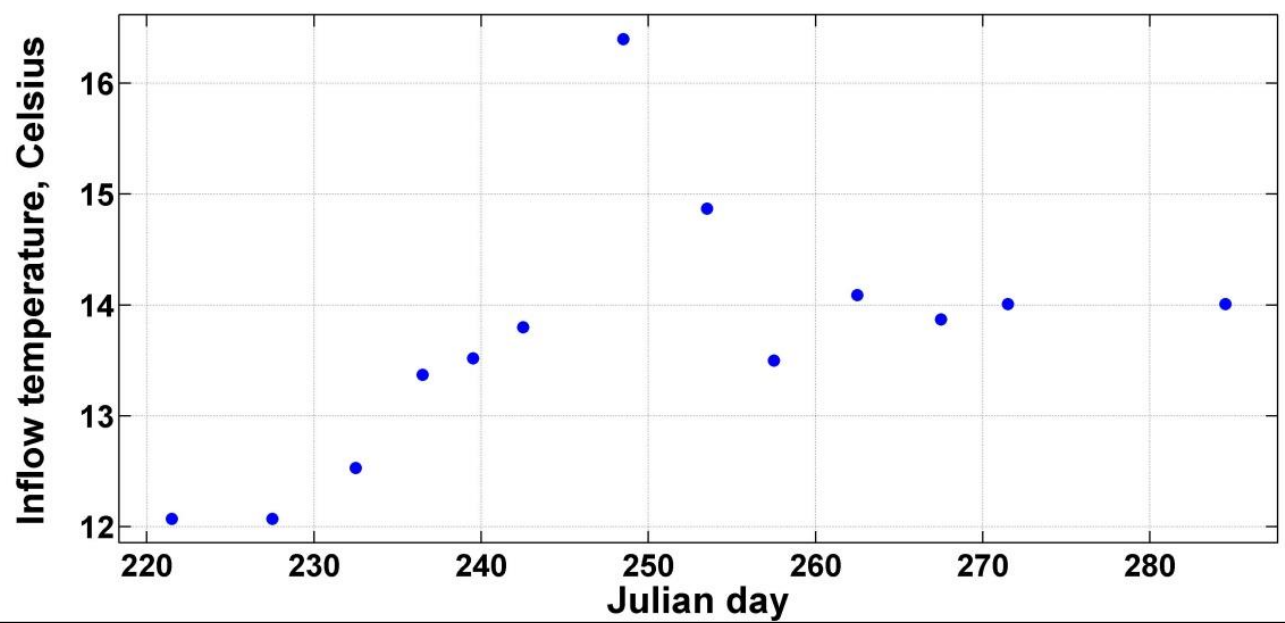

Figure 4-8. Lake Chaplain inflow temperature 


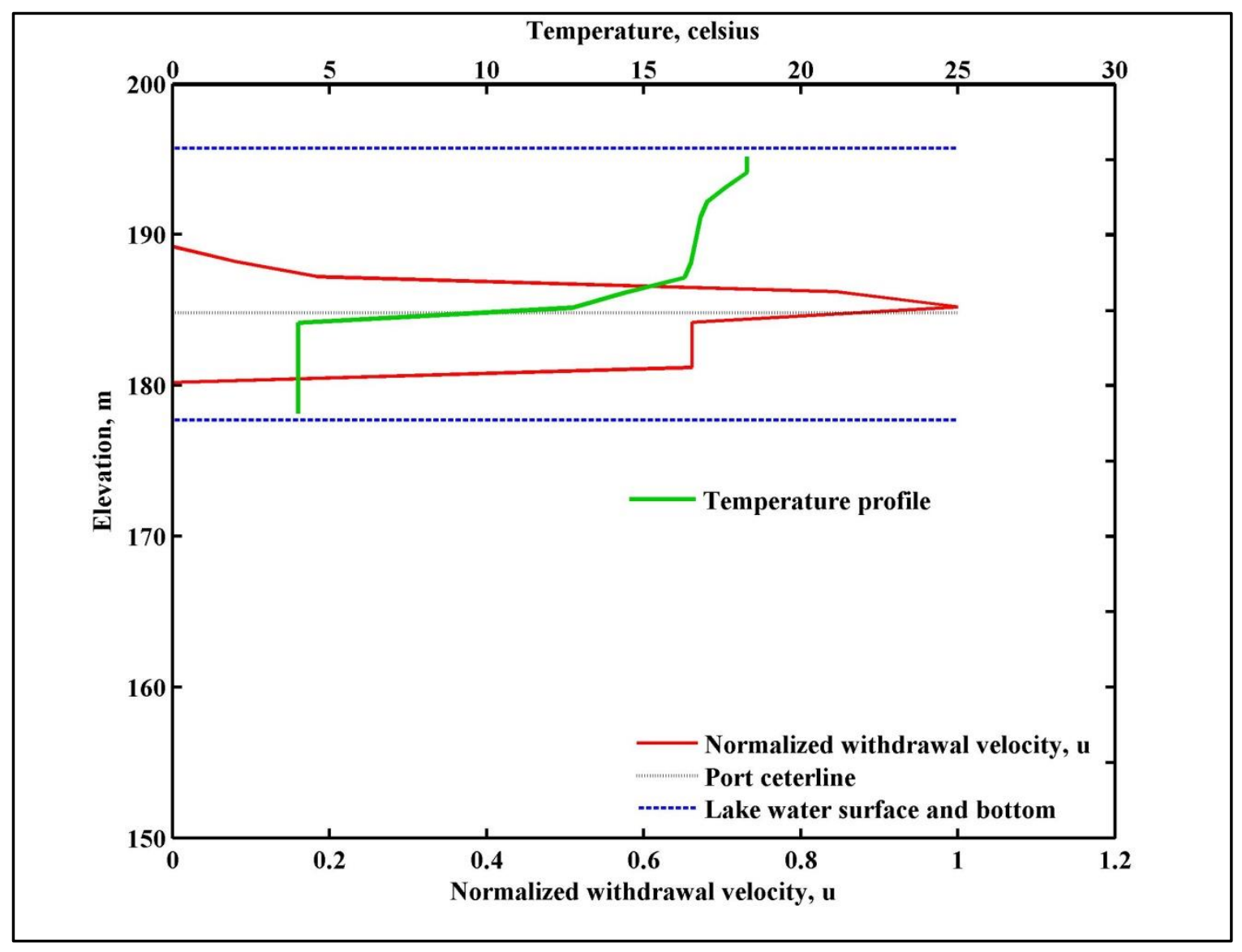

Figure 4-9. Initial temperature profile and normalized u-velocity at the dam withdrawal 


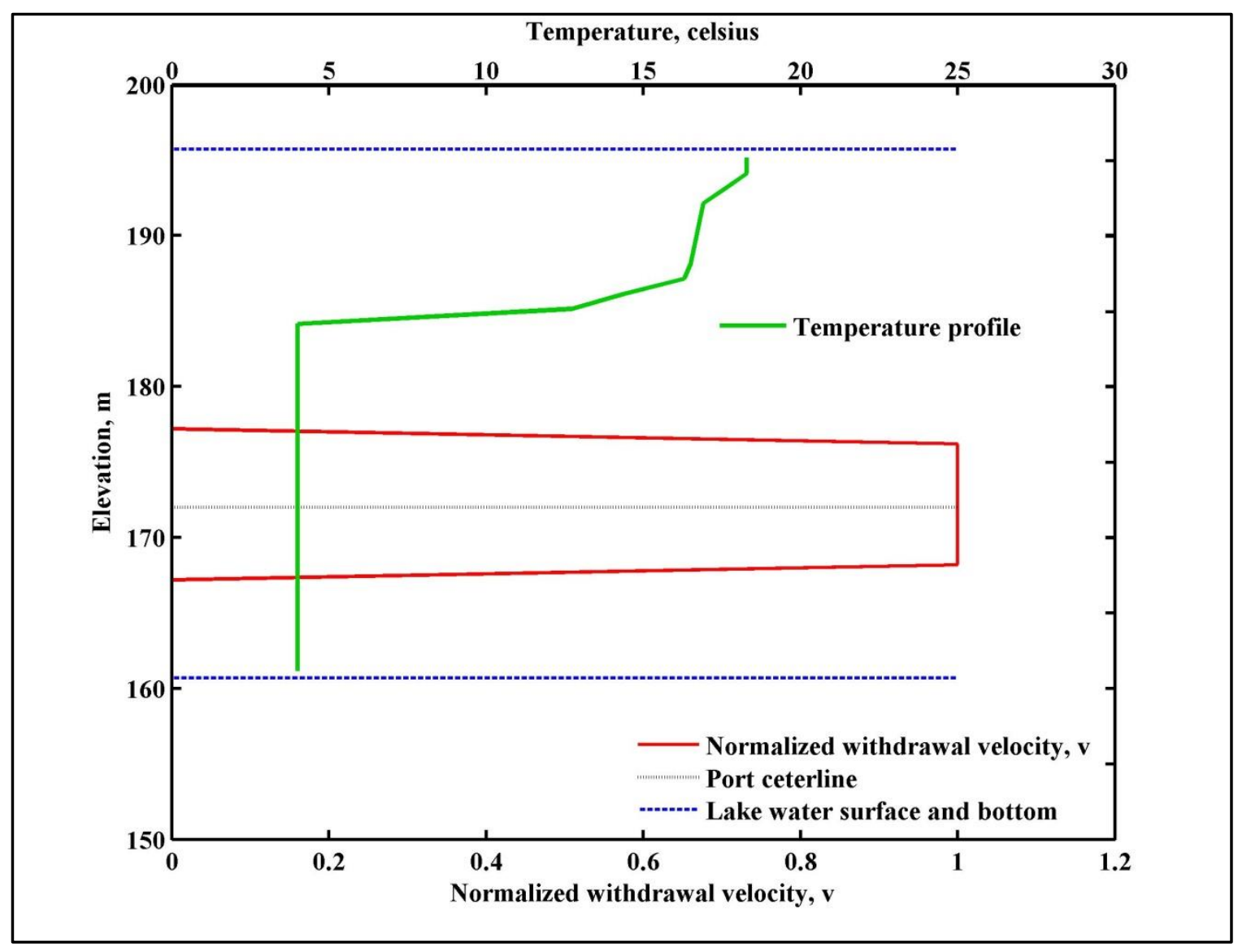

Figure 4-10. Initial temperature profile and normalized v-velocity at the paper mill withdrawal 


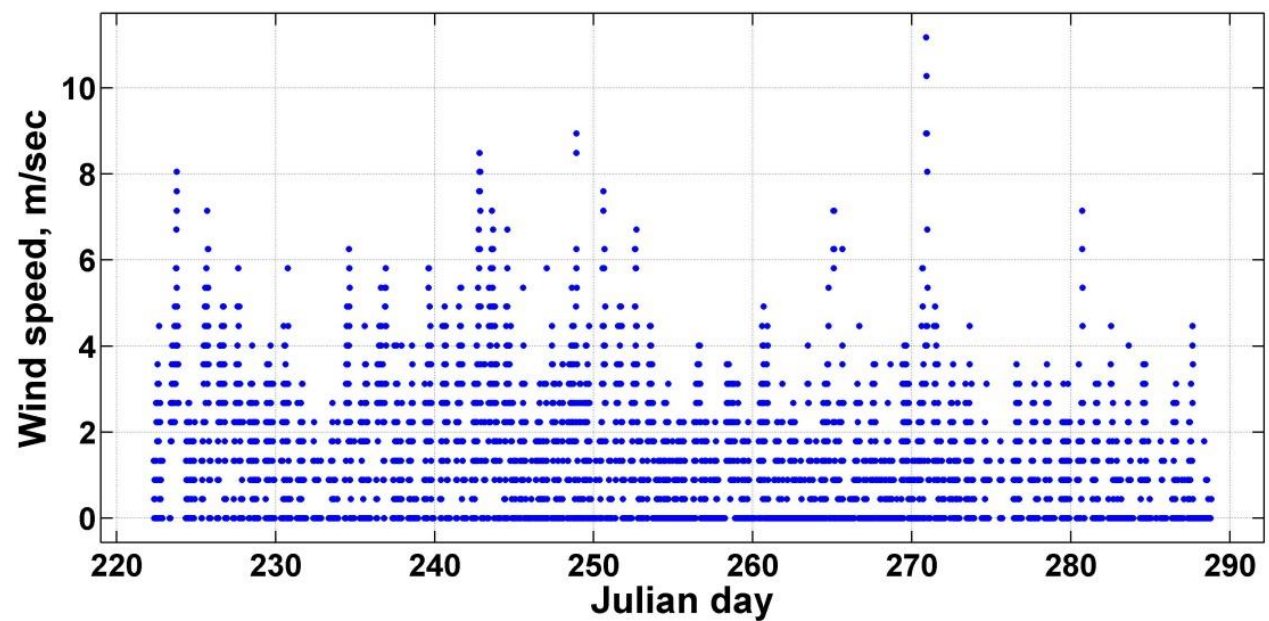

Figure 4-11. Lake Chaplain wind speed input data

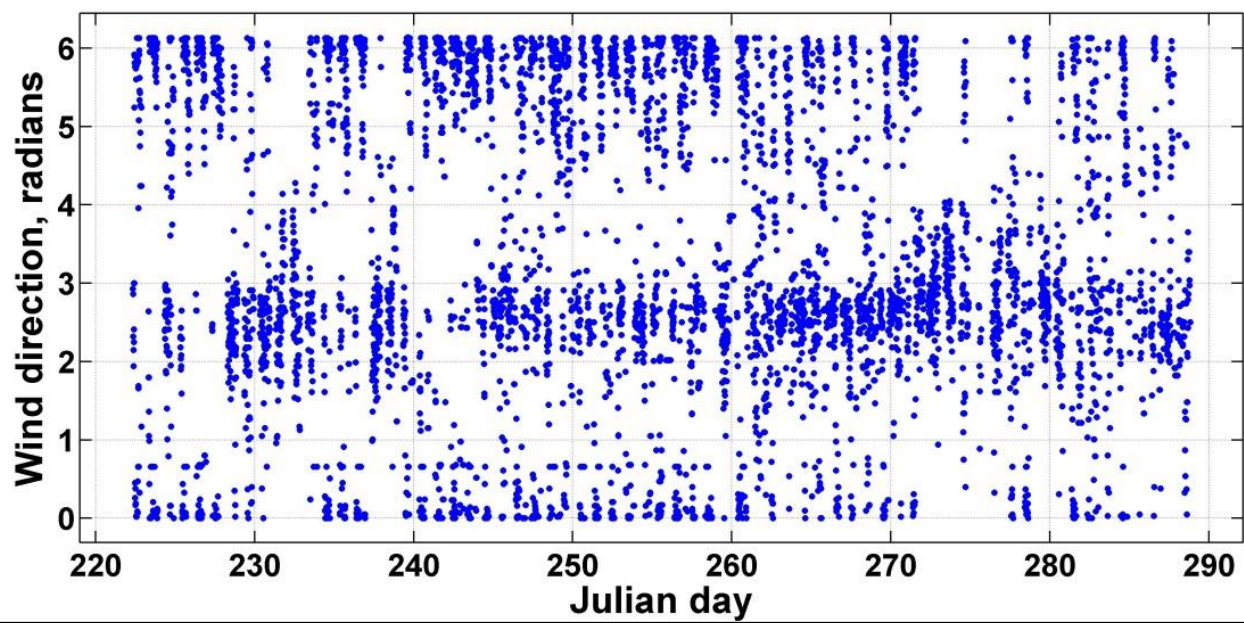

Figure 4-12. Lake Chaplain wind direction input data 


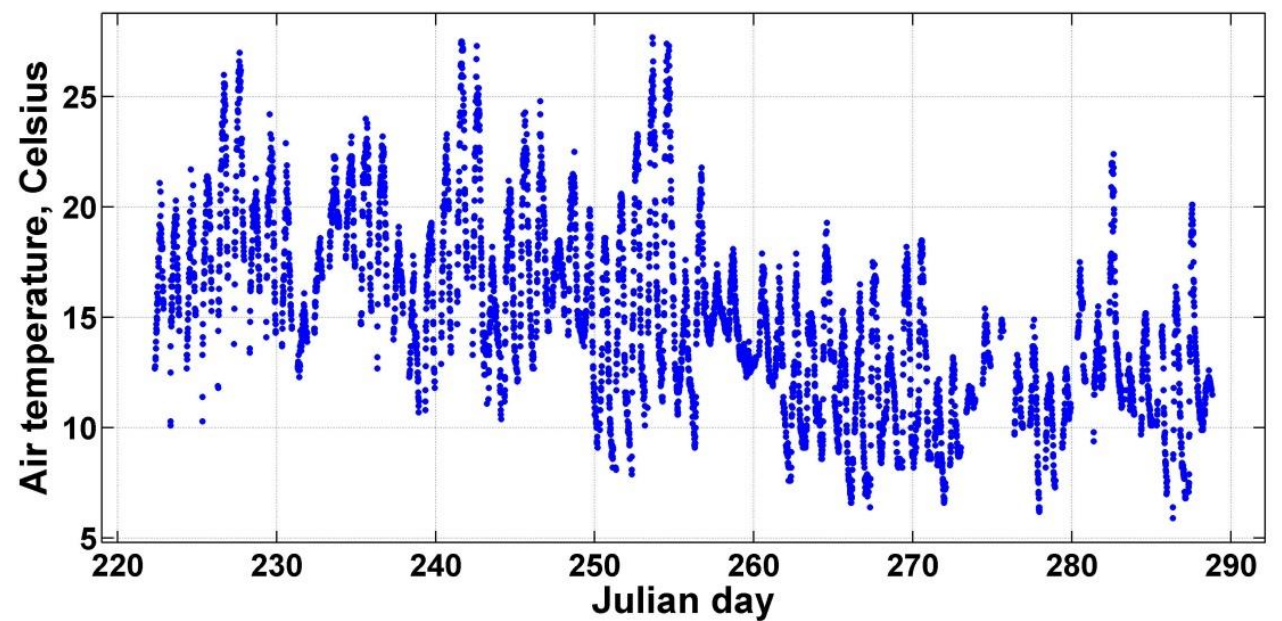

Figure 4-13. Lake Chaplain air temperature input data

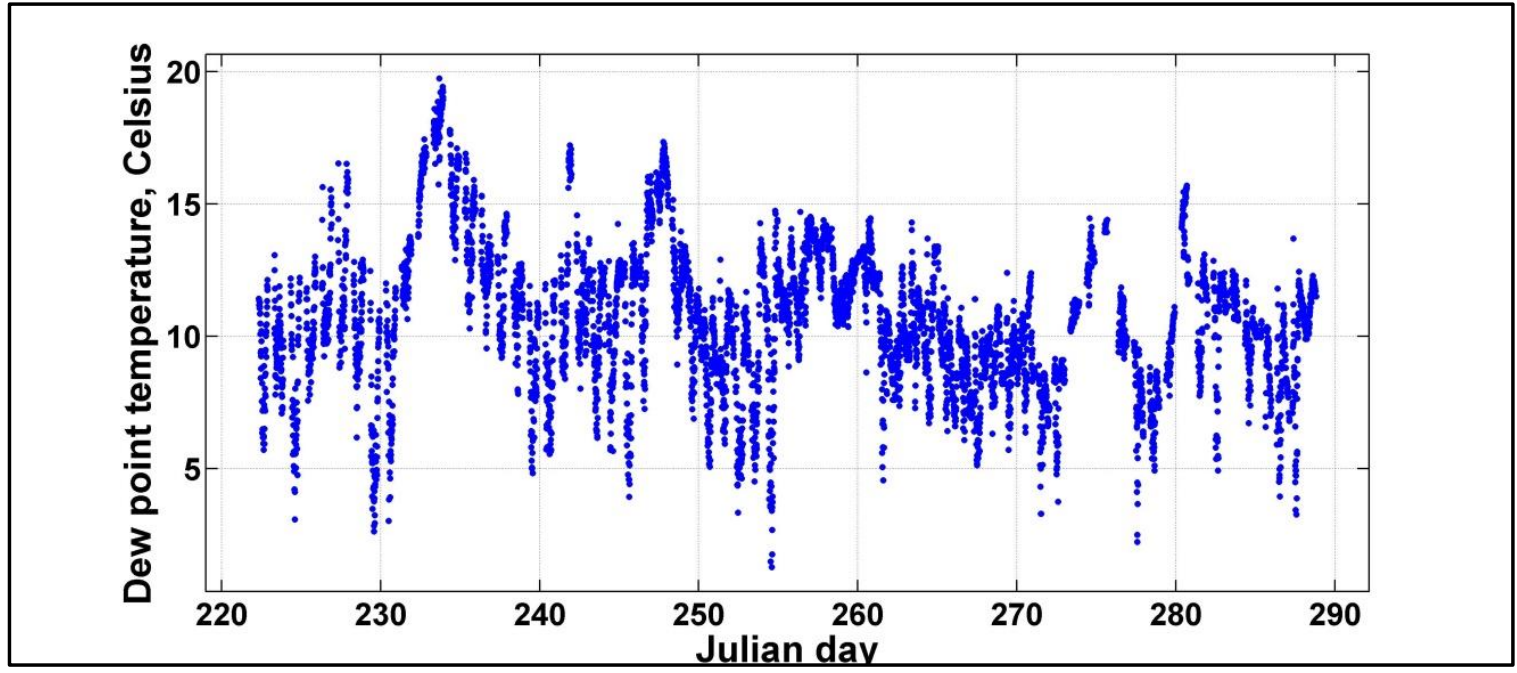

Figure 4-14. Lake Chaplain dew point temperature input data 


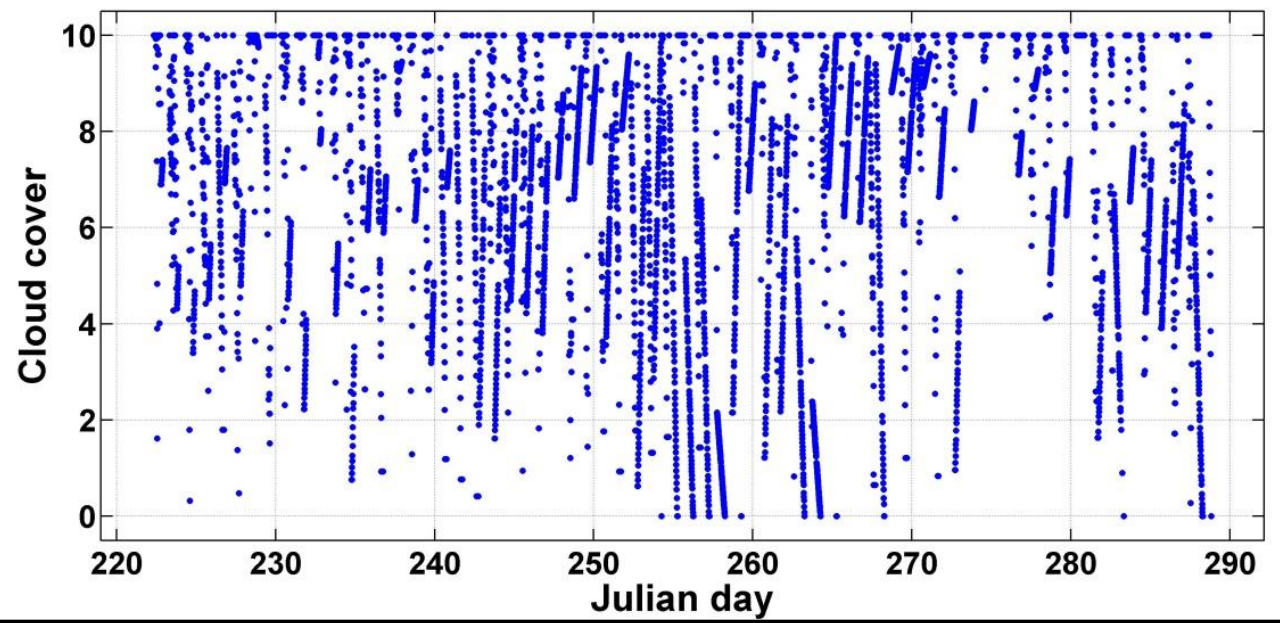

Figure 4-15. Lake Chaplain cloud cover input data

\subsubsection{Lake Chaplain Model Calibration Results}

The model was calibrated over a period of time from Aug10, 2007 to Oct11, 2007 (Julian day: 222 - 284) by using semi-implicit scheme $(\theta=0.55)$ for calculating free surface elevations and a time step of $5 \mathrm{sec}$. The simulation was performed starting from the initial water surface elevation of the available data, $195.73 \mathrm{~m}$ at the dam at Julian day of 222.35 $\mathrm{m}$. The wind-sheltering confident (WSC) was adjusted depending on the water levels and thermal vertical mixing. As a result, a WSC range between 0.5 and 0.85 was used during the model calibration. A comparison in water surface levels between model predictions and data is shown in Figure 4-16. Such a comparison is necessary to check and verify the water balance accuracy. The model results showed good agreement with the field data. Water surface error statistics showed that using the UPWIND scheme with WSC of 0.8 , the root mean squared error (RMSE) was $0.079 \mathrm{~m}$ and the absolute mean error (AME) was $0.065 \mathrm{~m}$. 


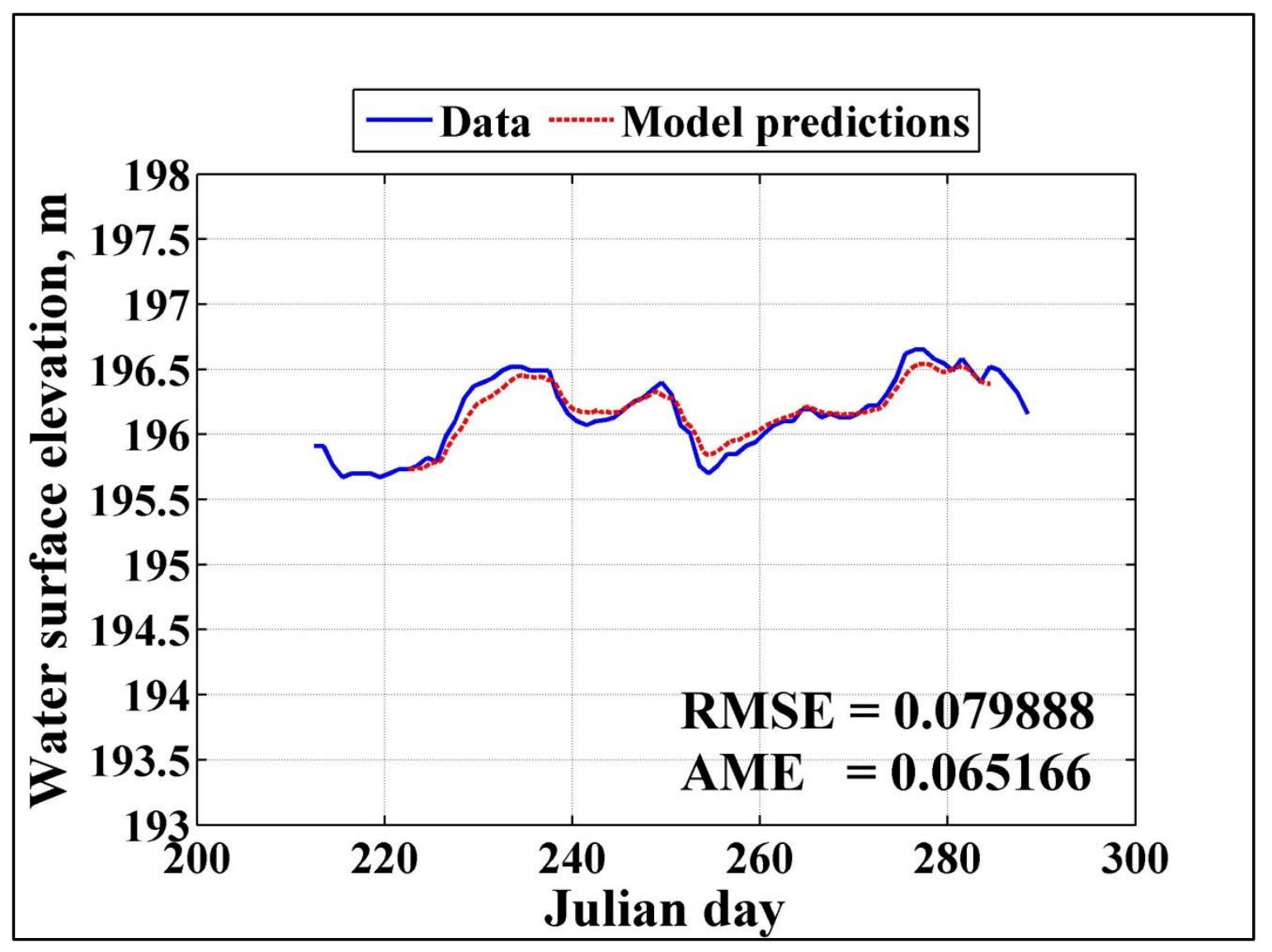

Figure 4-16. Lake Chaplain model predictions of water surface elevation compared with data using UPWIND scheme and WSC of 0.8

The velocity measurements were at model location of $i=11$ and $j=5$ at water depth of 6,8 , 10, 12, and $14 \mathrm{~m}$, measured from the water surface (see Figure 4-3). Therefore, we assumed velocities data measurements are in the $x$-direction, and we compared the present 3D model predictions of velocities with the data. Comparisons were done between the model and data to show that the model predictions of velocities are of the same order of magnitude as data if both of them were in the same direction. Figure 4-17 to Figure 4-21 are the model results at various depths over a period of time (Julian day: 222 - 284). 


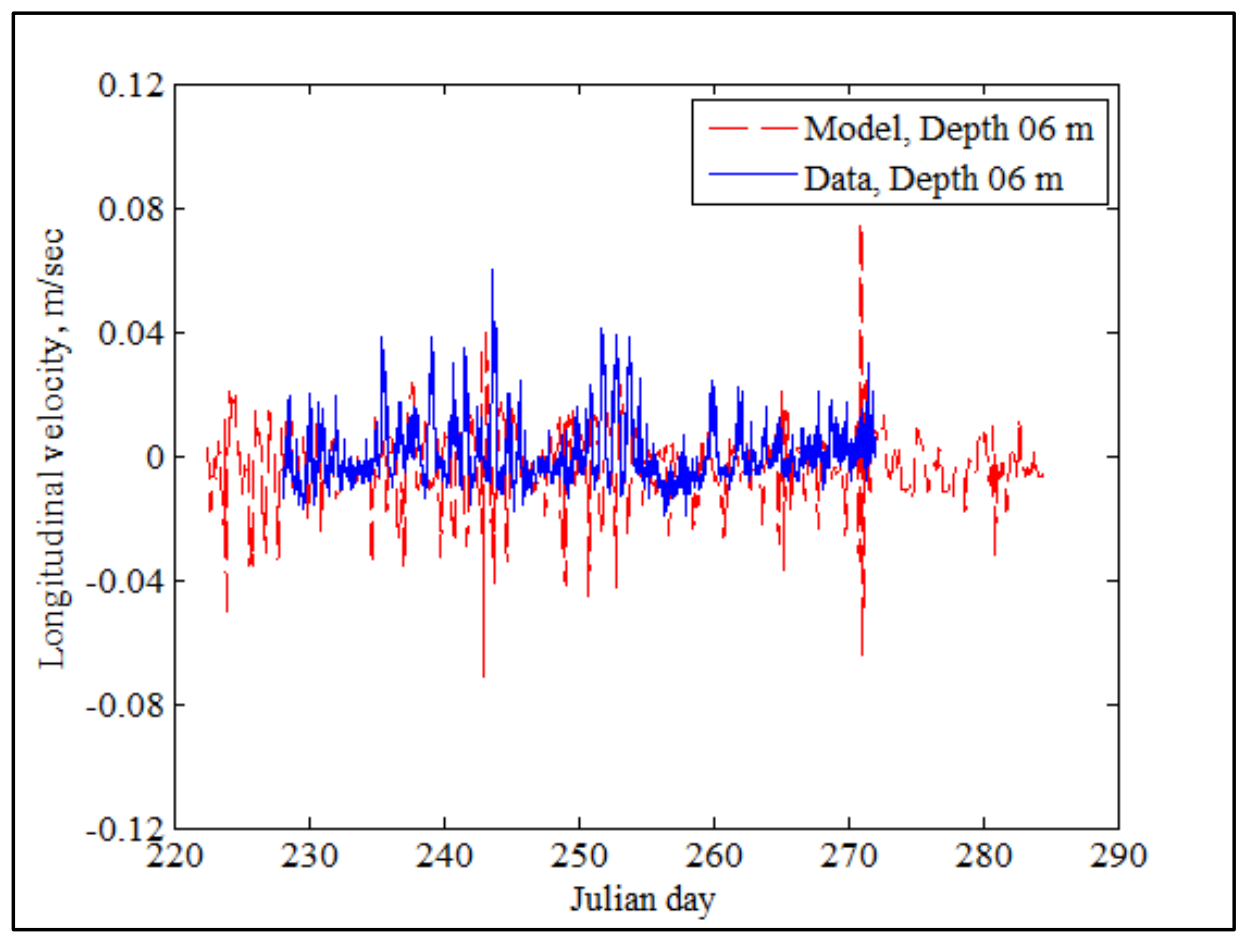

Figure 4-17. Comparison between Lake Chaplain model predictions of velocities with data at $i=11$ and $j=5$ at $6 \mathrm{~m}$ depth

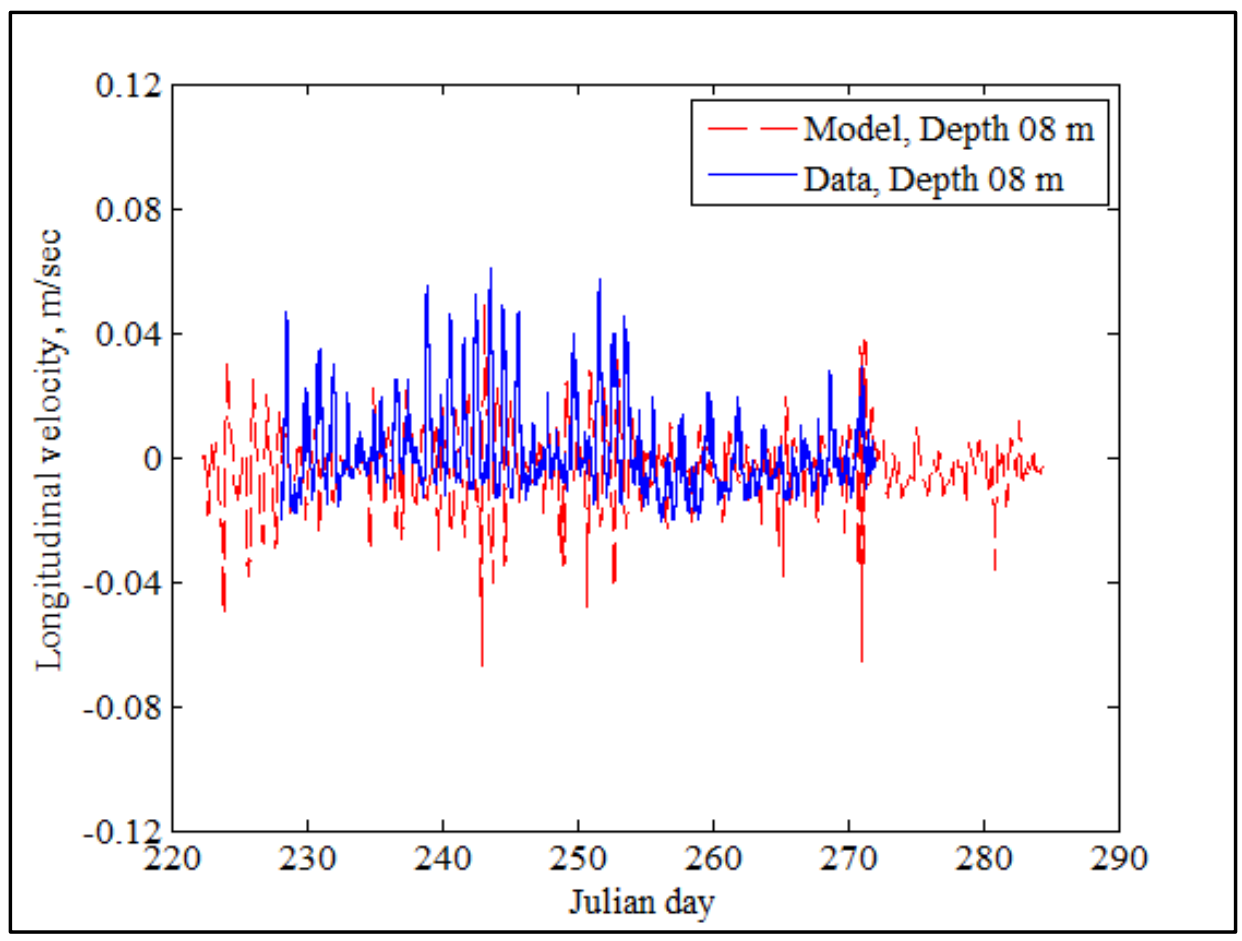

Figure 4-18. Comparison between Lake Chaplain model predictions of velocities with data at $i=11$ and $j=5$ at $8 \mathrm{~m}$ depth 


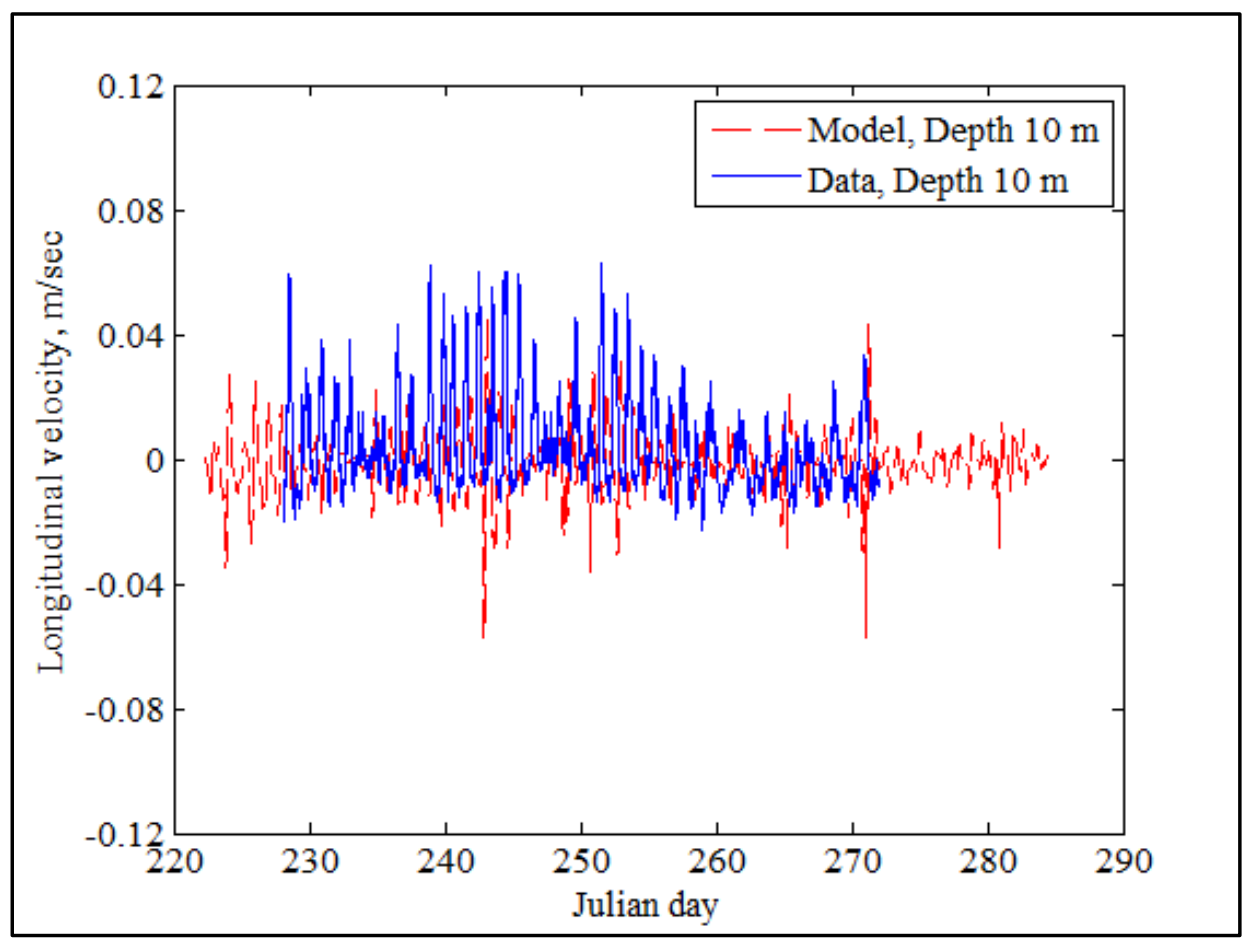

Figure 4-19. Comparison between Lake Chaplain model predictions of velocities with data at $i=11$ and $j=5$ at $10 \mathrm{~m}$ depth

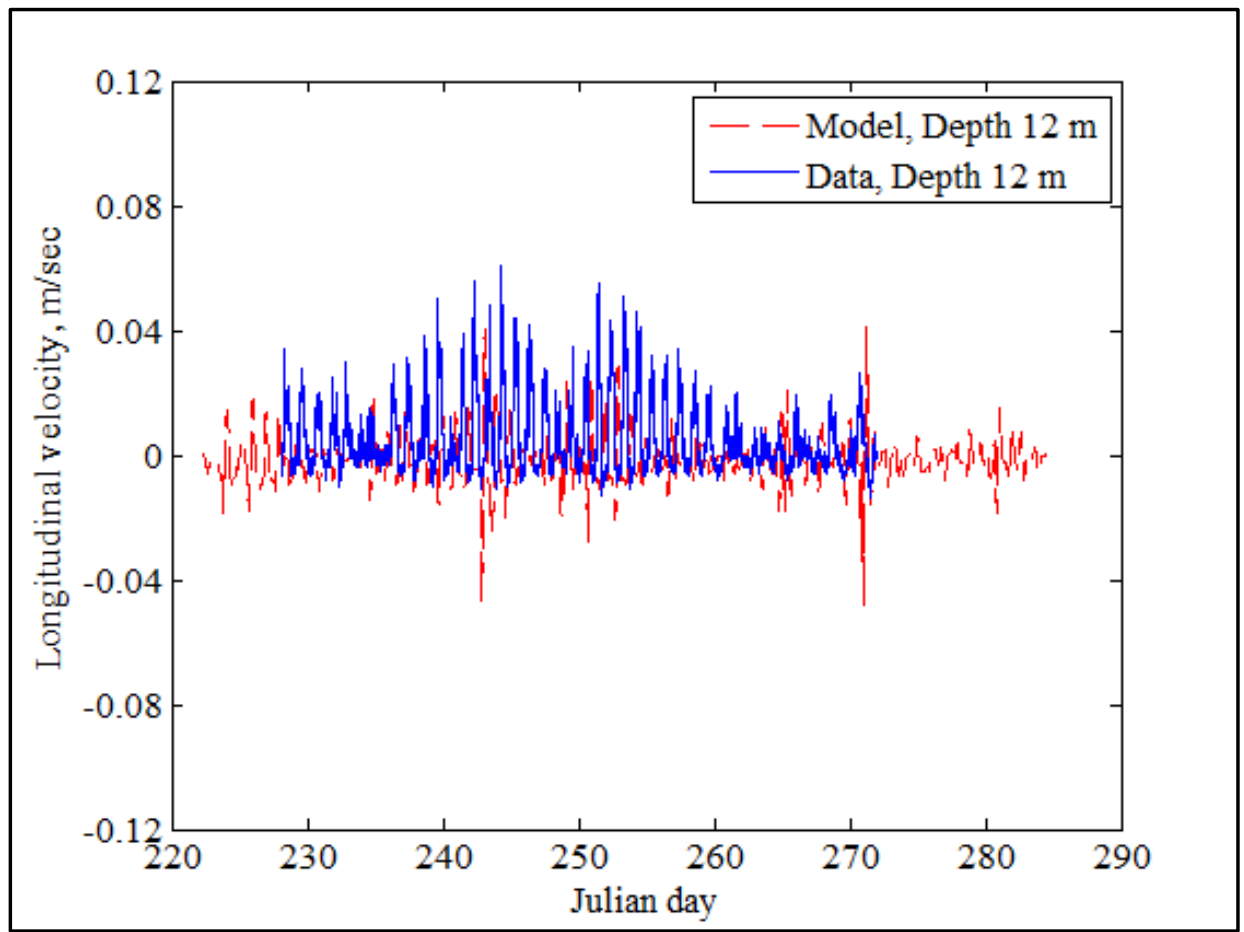

Figure 4-20. Comparison between Lake Chaplain model predictions of velocities with data at $i=11$ and $j=5$ at $12 \mathrm{~m}$ depth 


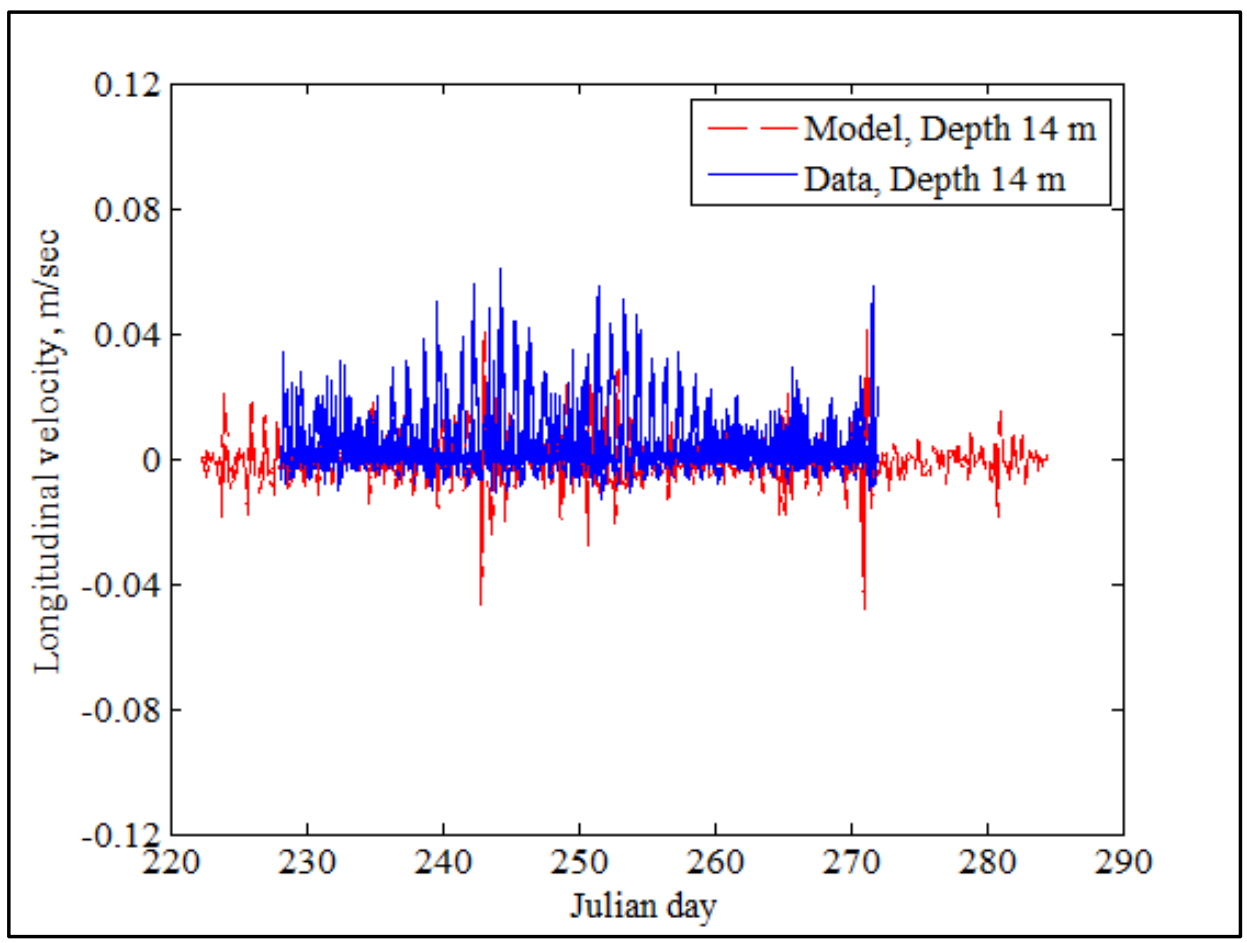

Figure 4-21. Comparison between Lake Chaplain model predictions of velocities with data at $i=11$ and $j=5$ at $12 \mathrm{~m}$ depth

The main target was to match the temperature profile data with the model profile predictions at various times along the simulation period. The available data were at model location of $i=11$ and $j=5$ at Julian days $(227.5,232.5,236.5,239.5,242.5,248.5,253.5$, $257.5,267.5$, and 271.5). The water temperature calibration was done by adjusting the temperature calibration parameters (Light extinction coefficient, evaporation wind speed function coefficients $(a, b$, and $c)$, percent of light absorbed at the water surface, and wind-sheltering coefficient). These parameters can be adjusted for calibrating temperature (Cole \& Wells, 2017). As a result, the model predictions of temperature in Lake Champlain model were primarily sensitive to the wind velocity on the lake. Thus, 
increasing wind speed leads to more vertical mixing and evaporation, cooling the water surface.

The model was run using water temperature parameters of $(0.25$ for extinction coefficient, 4.0 for wind speed function coefficient $a, 0.45$ for percent of light absorbed at the water surface, and 0.7-1.0 for wind-sheltering coefficient). Figure 4-22 shows a comparison between the model predictions and data at various times using the UPWIND scheme and wind-sheltering coefficient of 0.85 for (Julian day $\leq 250)$ and 1.00 for (Julian day > 250). Figure 4-23 shows a similar comparison but using a different wind-sheltering coefficient, 0.7 for $(228 \leq$ Julian day $\leq 258)$ and 0.8 for any time else.

The complete model simulation time (Julian day: 222 - 284) occurs during the stratification period in summer. Thus, we expect to see a sharp front temperature profile at the thermocline level. The model did well in matching data, but the model predictions of temperature have a smooth curve shape at the profile edge at the thermocline level as a result of using the UPWIND scheme for the heat advective transport. The smooth pattern at the thermocline is attributed to the UPWIND scheme which has significant numerical diffusion. The UPWIND scheme gave results not as accurate as the higher order schemes at the edge of a sharp front or gradient where there is a temperature discontinuity in the numerical solution predictions.

In order to improve the results, the model was run by using the higher order schemes (QUICK and QUICKEST) for the heat advective transport. The model predictions in Figure 4-22 and Figure 4-23 were determined by implementing QUICK and QUICKEST 
scheme and by using the same calibration parameters and input data (see Figure 4-24 to Figure 4-27). The calibration comparisons showed the model ability to predict temperatures at this site location was to within AME of 0.50875 to $1.88443{ }^{\circ} \mathrm{C}$ using QUICKEST scheme, 0.53944 to $1.5473{ }^{\circ} \mathrm{C}$ using QUICK scheme, and 1.0607 to 2.0443 ${ }^{\circ} \mathrm{C}$ using the UPWIND scheme (see Table 1 for the overall AME's). It is clear that the higher order schemes produced a sharp gradient.

In conclusion, the present 3D numerical model was applied to simulate hydrodynamics and temperature transport in Lake Chaplain. The model predictions were compared with field data to test that the model reasonably predicted water level, velocity, and temperature profiles. The comparison error statistics showed reasonable agreement, reflecting the model's ability to predict water levels, velocities, and temperatures successfully. The results indicated that higher-order schemes are important for temperature and water quality predictions.

In terms of the comparison with the 2D model, this case study was already modeled using the 2D CE-QWAL-W2 model and the 2D error statistics were summarized in Table 2. Also, the 2D water level and a few selected vertical temperature profiles as an example are shown in Figure 4-69 and Figure 4-49, respectively. 

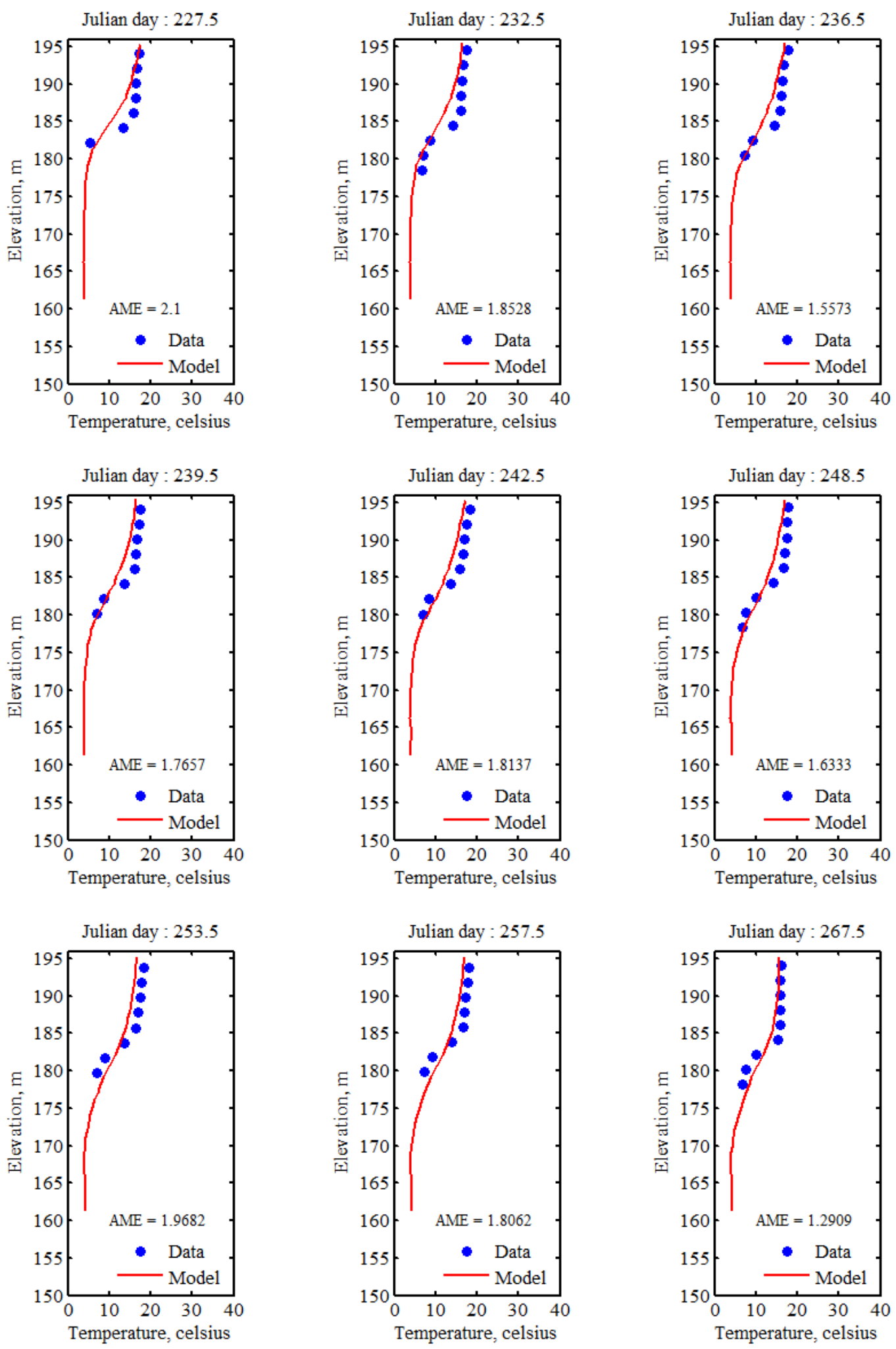


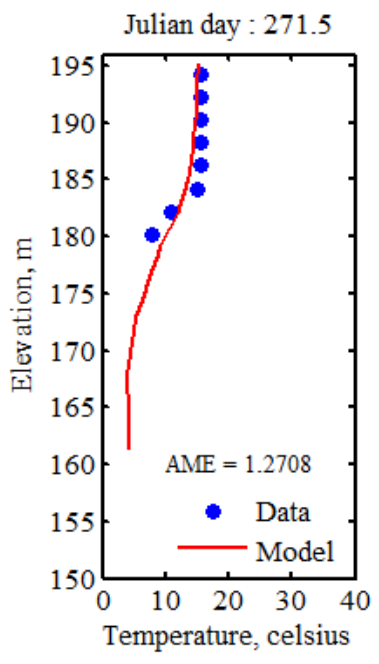

Figure 4-22. Lake Chaplain model predictions of temperature vertical profile compared with data using UPWIND scheme and WSC of 0.85 for (Julian day $\leq 250$ ) and 1.00 for (Julian day > 250) 

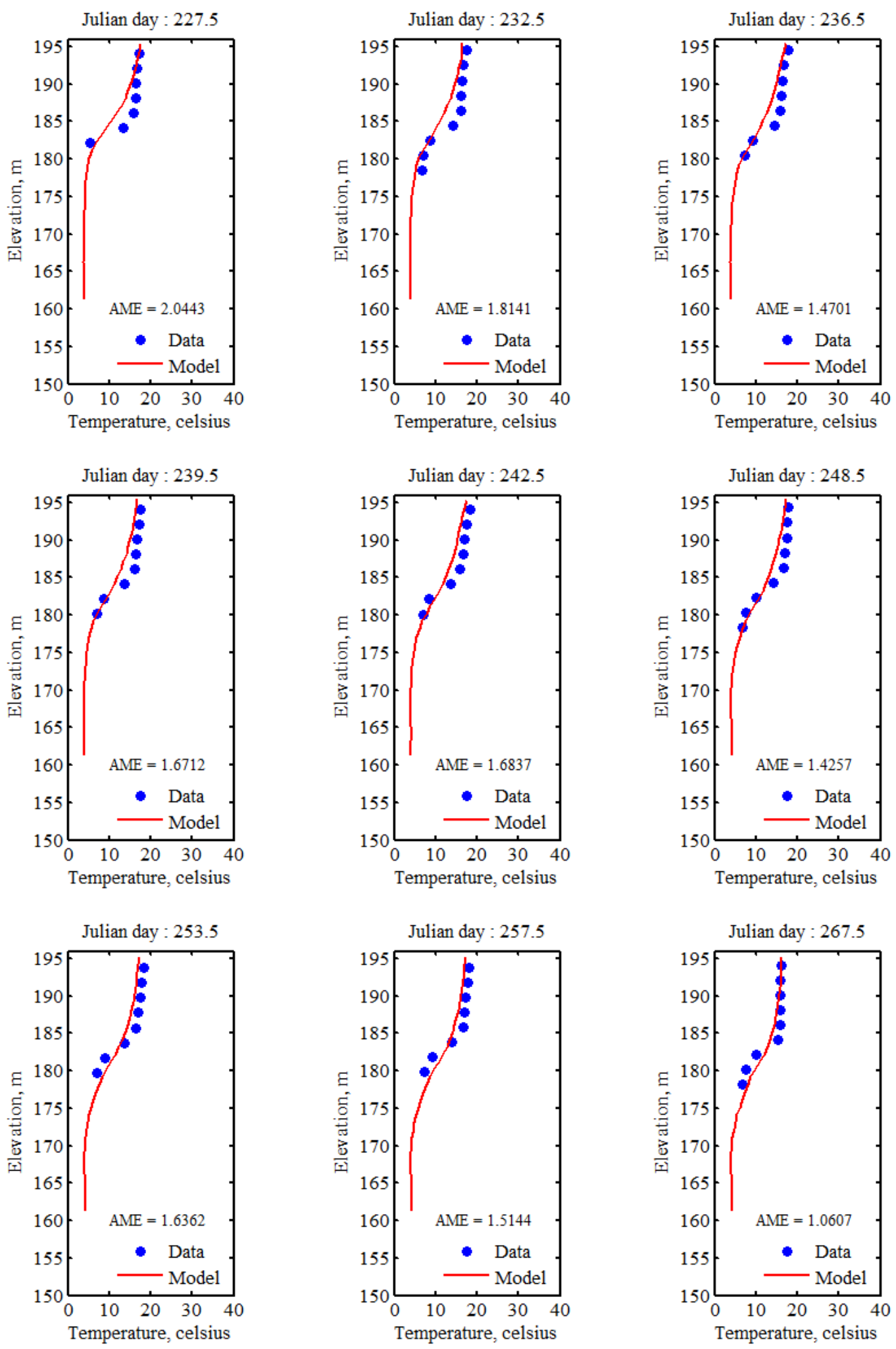


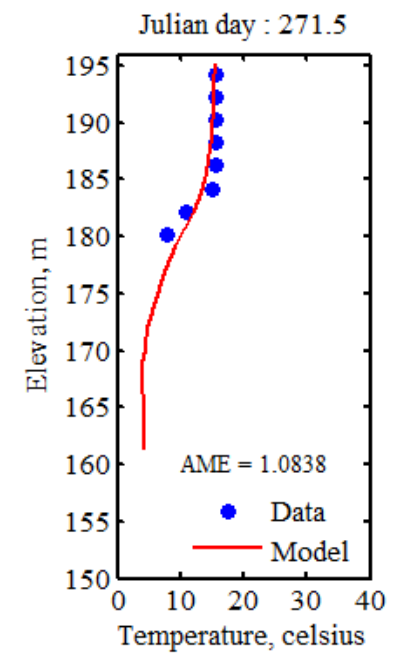

Figure 4-23. Lake Chaplain model predictions of temperature vertical profile compared with data using UPWIND scheme and WSC of 0.7 for $(228 \leq$ Julian day $\leq 258)$ and 0.8 for any time else 

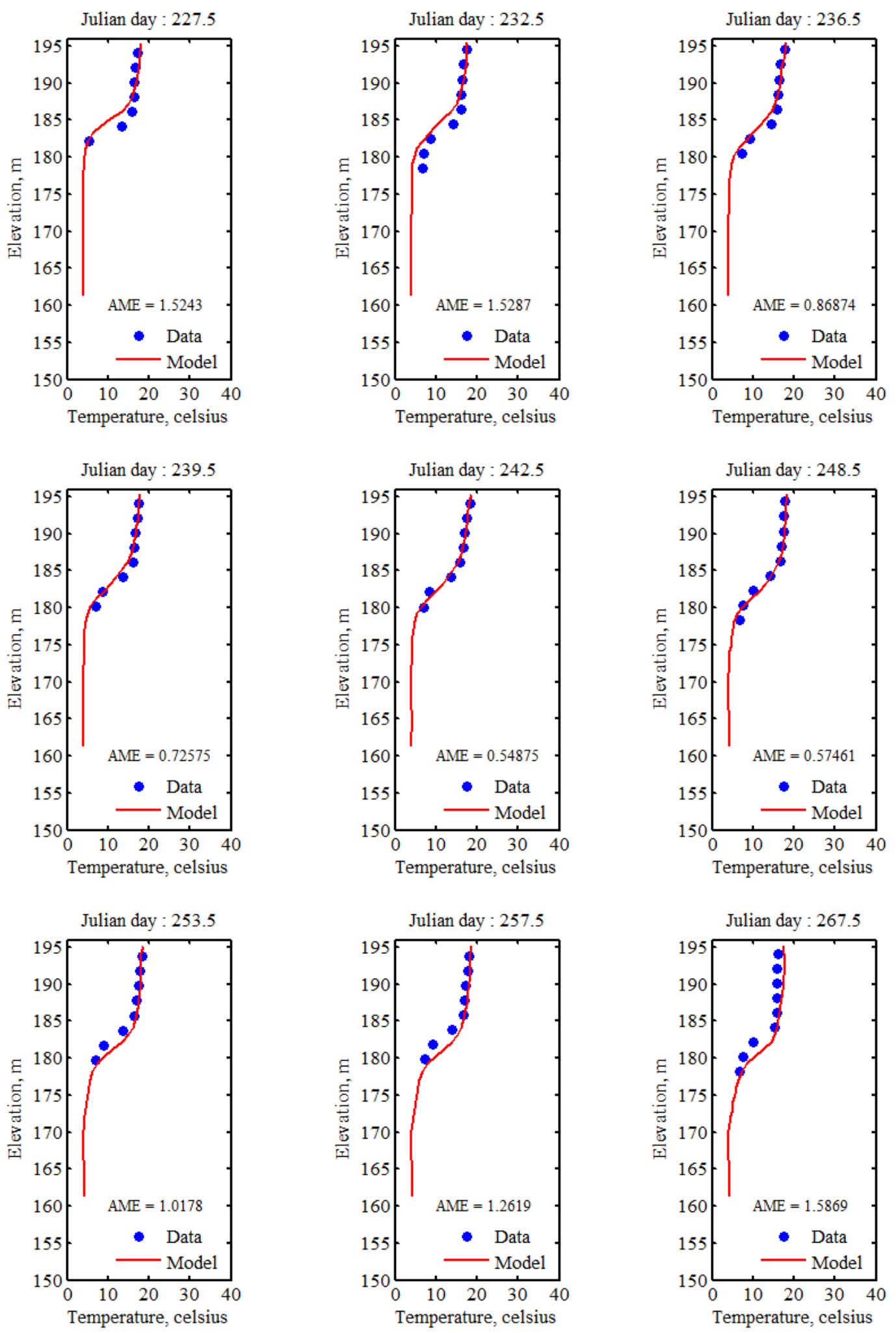


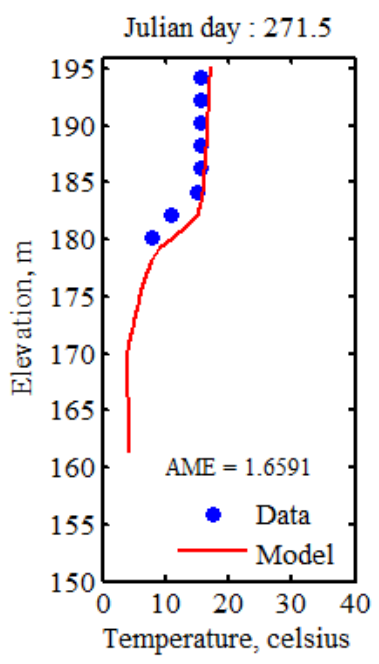

Figure 4-24. Lake Chaplain model predictions of temperature vertical profile compared with data using QUICK scheme and WSC of 0.85 for (Julian day $\leq 250$ ) and 1.00 for (Julian day > 250) 

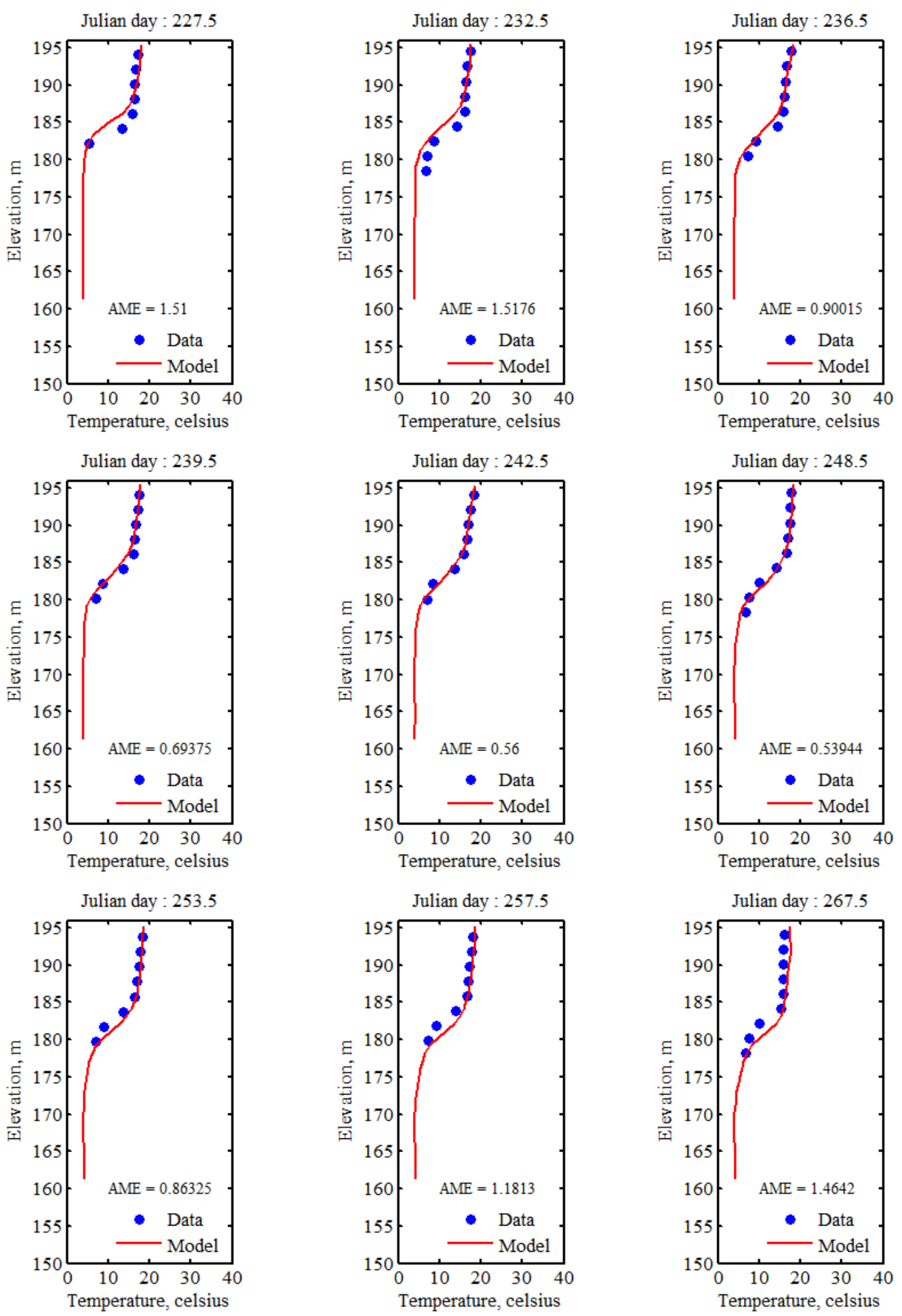


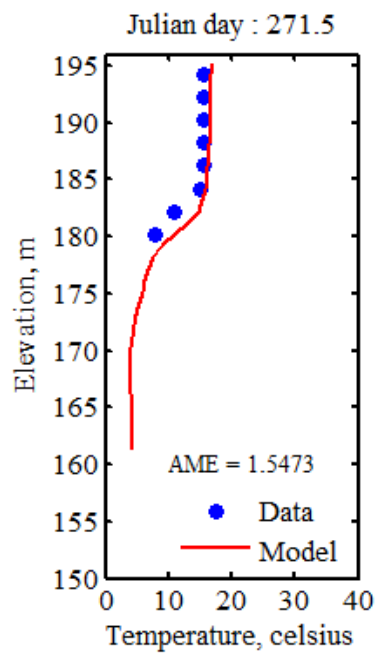

Figure 4-25. Lake Chaplain model predictions of temperature vertical profile compared with data using QUICK scheme and WSC of 0.7 for $(228 \leq$ Julian day $\leq 258)$ and 0.8 for any time else 

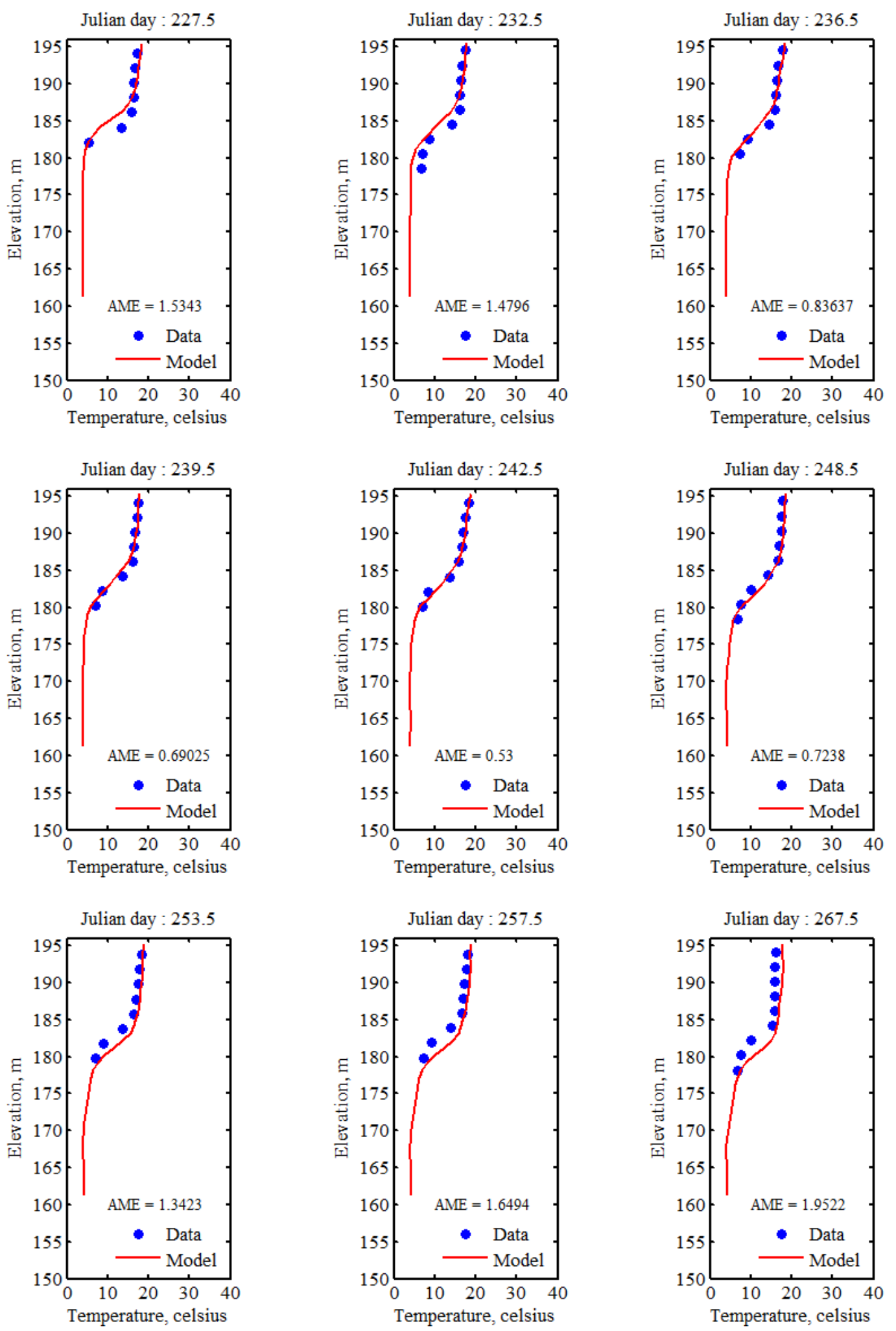


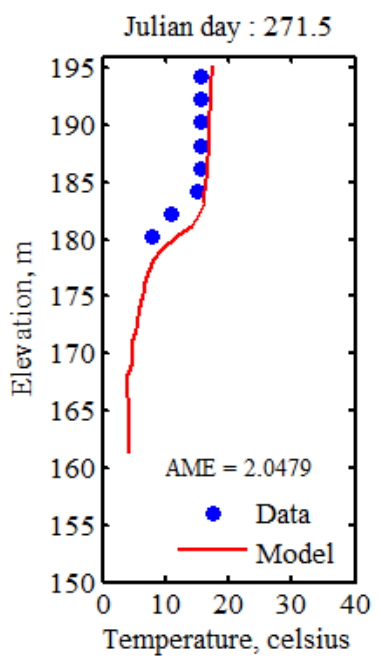

Figure 4-26. Lake Chaplain model predictions of temperature vertical profile compared with data using QUICKEST scheme and WSC of 0.85 for (Julian day $\leq 250$ ) and 1.00 for (Julian day > 250) 

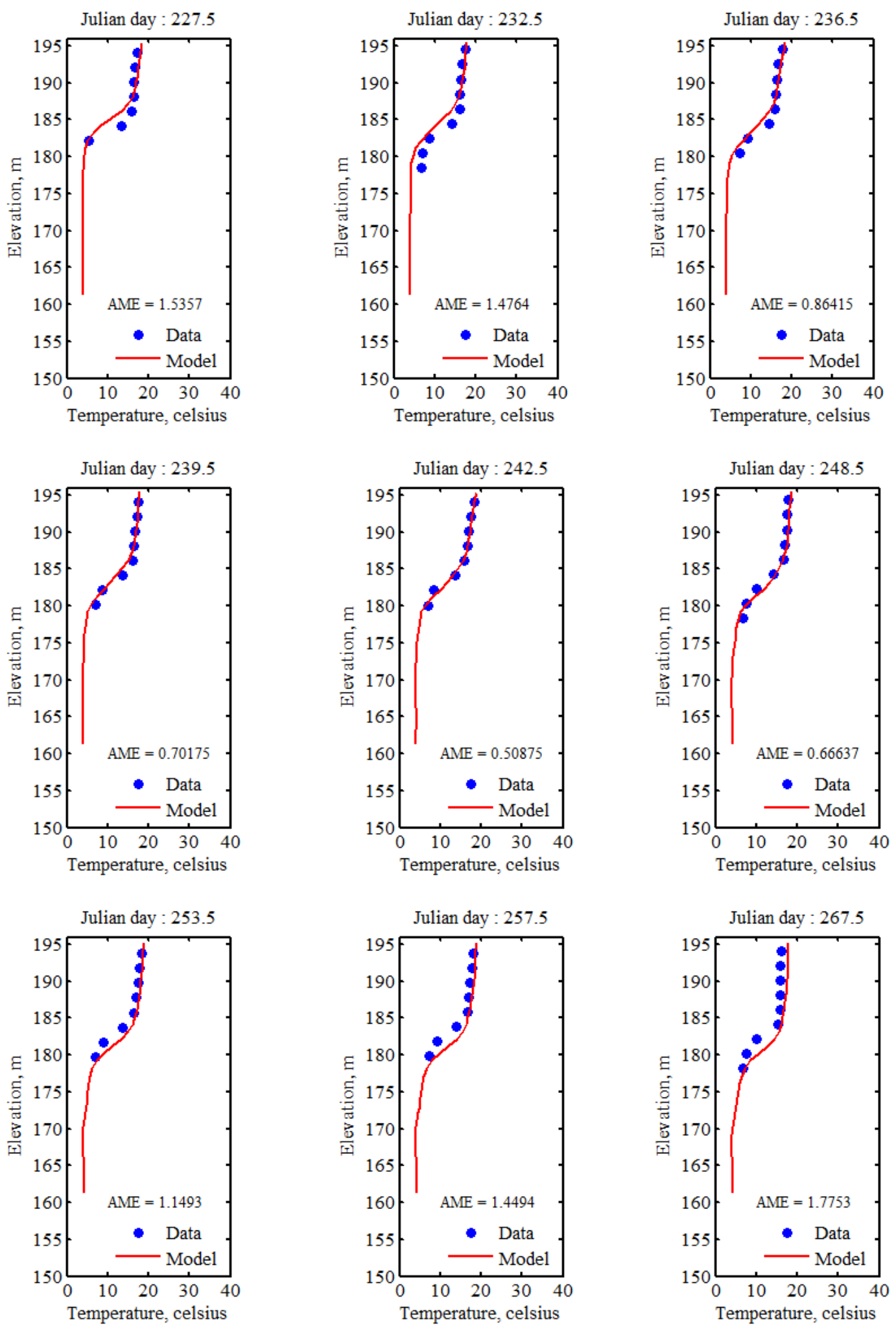


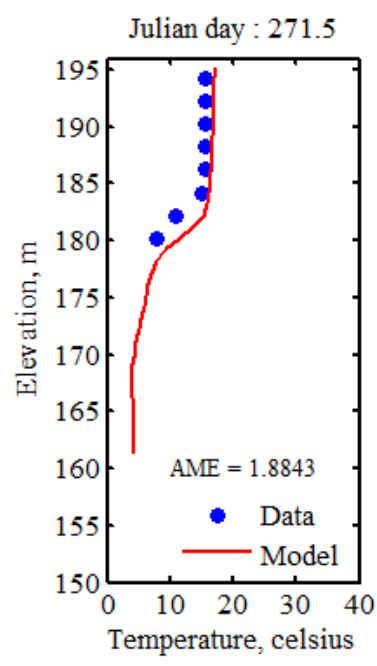

Figure 4-27. Lake Chaplain model predictions of temperature vertical profile compared with data using QUICKEST scheme and WSC of 0.7 for $(228 \leq$ Julian day $\leq 258)$ and 0.8 for any time else

Table 1. Overall AME in ${ }^{\circ} \mathrm{C}$ based on $(7,8$, or, 9) comparisons for each temperature profile

\begin{tabular}{lccc}
\hline & UPWIND & QUICK & QUICKEST \\
\hline $\begin{array}{l}\text { WSC of } 0.85 \text { for (Julian } \\
\text { day } \leq 250) \text { and } 1.00 \text { for } \\
(\text { Julian day }>250) .\end{array}$ & 1.71 & 1.13 & 1.28 \\
$\begin{array}{l}\text { WSC of } 0.7 \text { for }(228 \leq \\
\text { Julian day } \leq 258) \text { and } 0.8\end{array}$ & 1.54 & & \\
for any time else & & 1.08 & 1.20 \\
\hline
\end{tabular}

Table 2. Overall AME of CE-QUAL-W2 numerical predictions of Lake Chaplain

\begin{tabular}{ccc}
\hline & Water Level, $\mathrm{m}$ & Temperature, ${ }^{\circ} \mathrm{C}$ \\
\hline AME & 0.018 & 0.360 \\
\hline
\end{tabular}




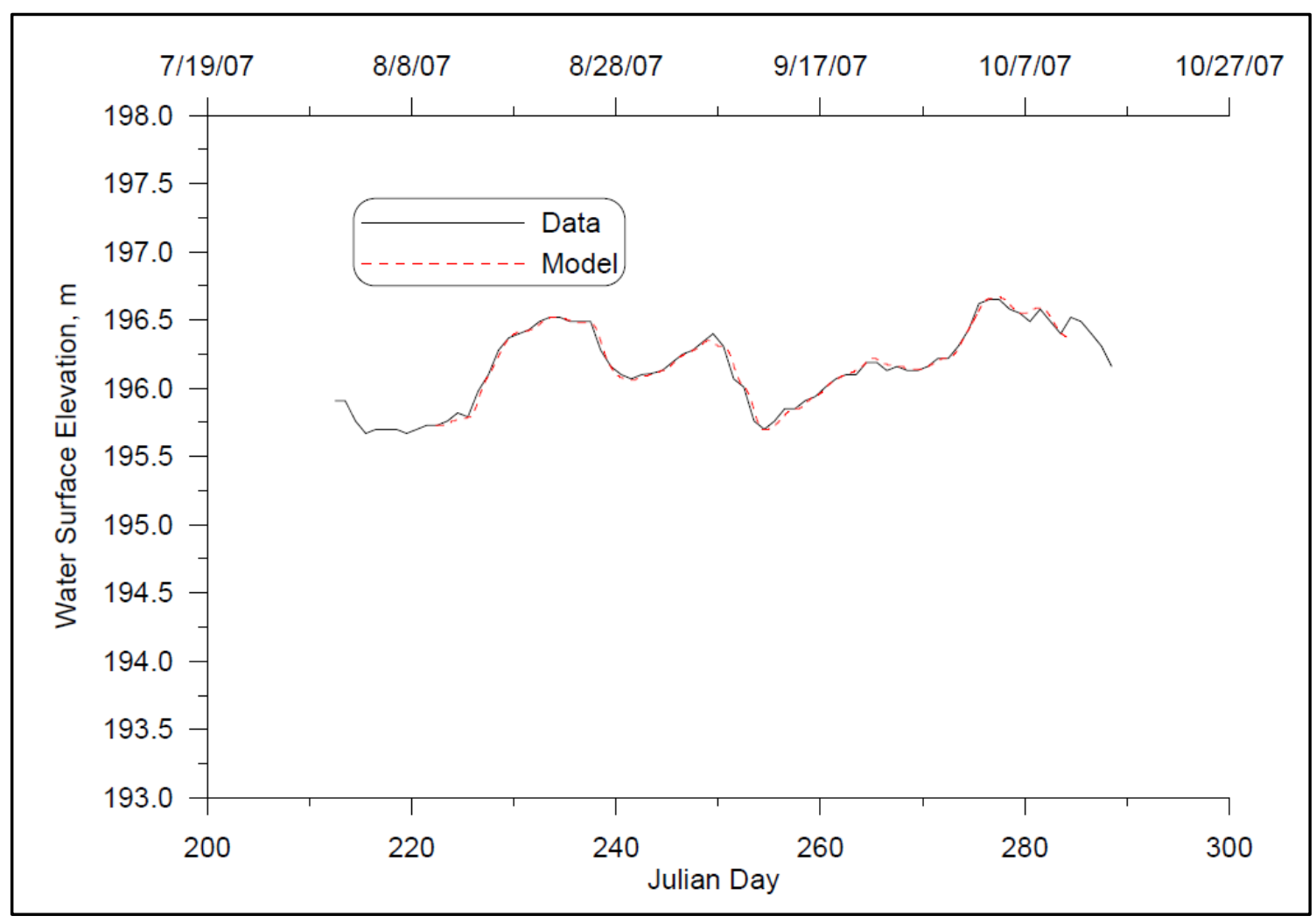

Figure 4-28. CE-QUAL-W2 water level numerical predictions of Lake Chaplain model compared to data

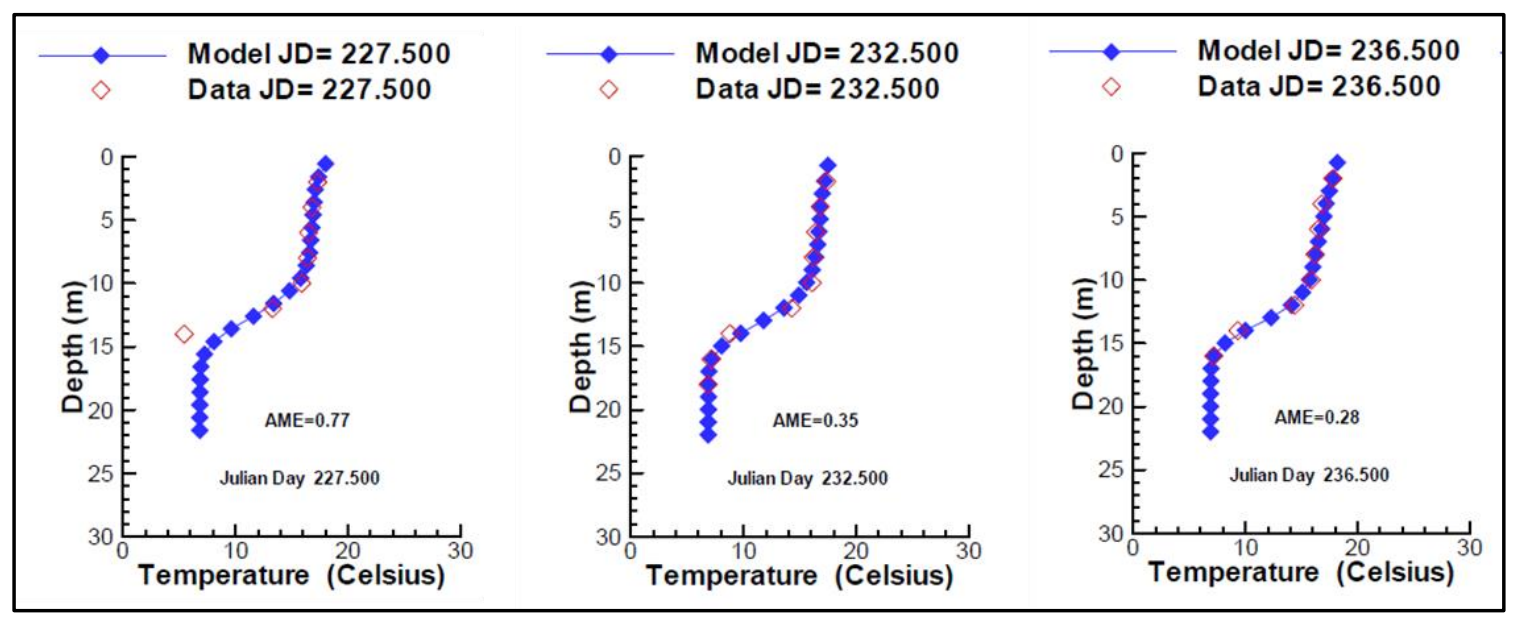

Figure 4-29. CE-QUAL-W2 vertical temperature profiles of Lake Chaplain model compared to data 


\subsection{Case Study 2: Laurance Lake - Comparison between the 2D and 3D Model}

A two and three-dimensional hydrothermal numerical model were developed and calibrated for Laurance Lake, Oregon, USA. The numerical scheme in the twodimensional laterally averaged hydrodynamic and water quality model, CE-QUAL-W2, was adapted for a three-dimensional model to solve the governing equations of continuity, free surface equation, and momentum. In order to perform an equivalent comparison between the results of the two and three-dimensional model, both models were run using the same meteorological data, boundary conditions of flow and temperature, and surface heat exchange algorithms. The reservoir water levels and temperature were calibrated over the simulation period. Even though the calculated error statistics of the 3D model were slightly higher compared to the 2D model, the numerical predictions of both models had good agreement with field data. Conditions where a $2 \mathrm{D}$ or a 3D model is better suited to a lake or reservoir were discussed. Furthermore, the inorganic suspended solids (ISS) vertical profiles were explored to show how water quality computations were coupled with hydrodynamics and temperature in the present model.

\subsubsection{The Study Background}

Researchers often must decide whether to use a 2D (longitudinal-vertical) or a 3D model for simulating stratified lakes and reservoirs. Many may decide that a 3D model is always superior to a 2D model because of it being '3D'. But, depending on the research questions that need to be answered both models may work, and both models have advantages and disadvantages. Computational time, management questions to be 
answered, and model complexity are often deciding factors in determining whether to use a $2 \mathrm{D}$ or 3D model. In order to assess differences in these model types, a 3D model was developed based on similar numerical scheme and algorithms as in the 2D CE-QUALW2 model (Cole \& Wells, 2017). The present 3D model was applied to a few reservoirs in the USA for testing the model validity, including Lake Chaplain, WA, USA (AlZubaidi \& Wells, 2017a); Laurance Lake, OR, USA (Al-Zubaidi \& Wells, 2018c); and Cooper Creek Reservoir, OR, USA (Al-Zubaidi \& Wells, 2018d). Comparisons between the model predictions of water level and vertical temperature profiles with field data were performed for the 2D and 3D model using the same model inputs and boundary conditions.

\subsubsection{Study Area Overview}

Laurance Lake is a reservoir located in Hood River County, Oregon State, US (latitude: 45.46, longitude: -121.66) with an approximate elevation of $910 \mathrm{~m}$ above sea level. An aerial view and a location map of Laurance Lake are shown in Figure 4-30 and Figure 4-31, respectively. The major inflow to the reservoir is from Clear Branch Creek at the reservoir's western end. A smaller inflow comes from Pinnacle Creek at the southeast corner of the reservoir. The dam is located at the east end of the reservoir where the outlet is located. The reservoir outlet is a pipe located at the bottom of the dam. Water is released through the pipe to maintain the minimum flow requirements of the Clear Branch Creek below the dam. The required input data, lake bathymetry, and data required for calibration were described in Berger et al.(2005). 


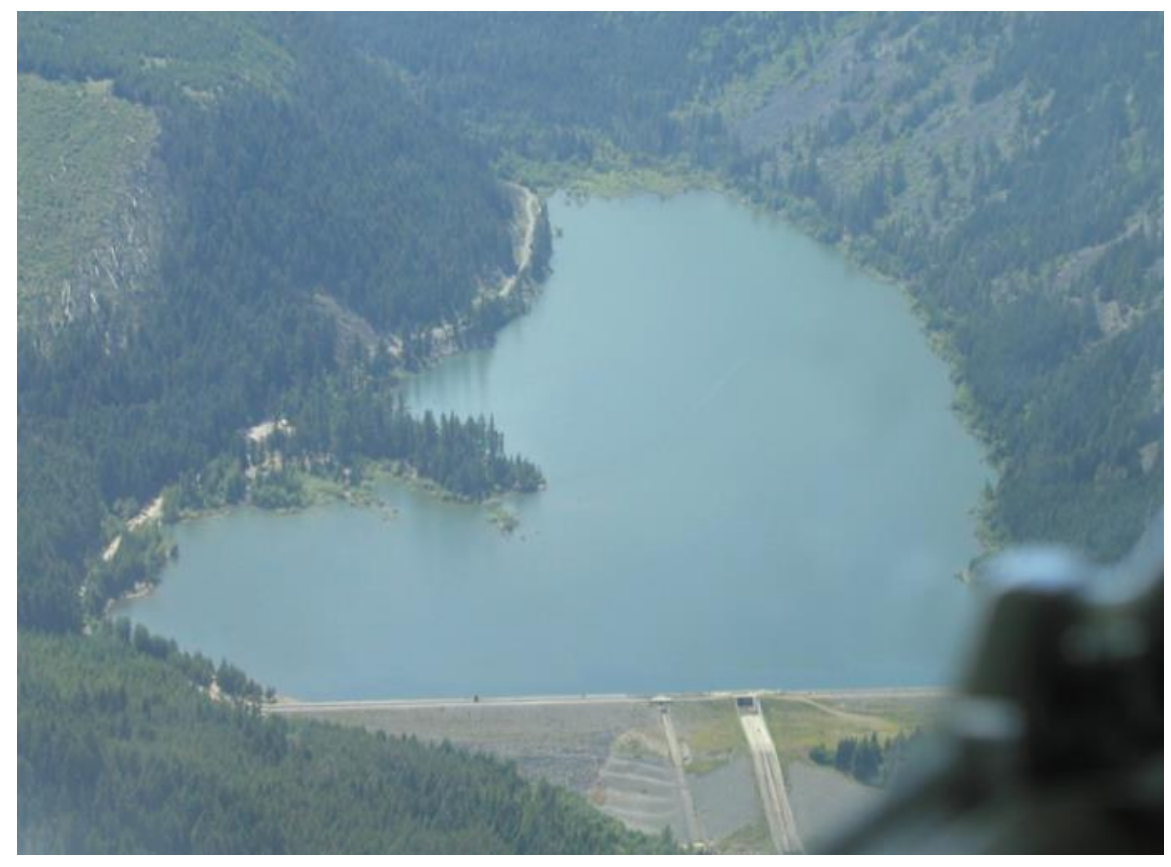

Figure 4-30. The Laurance Lake aerial view (Berger et al., 2005)

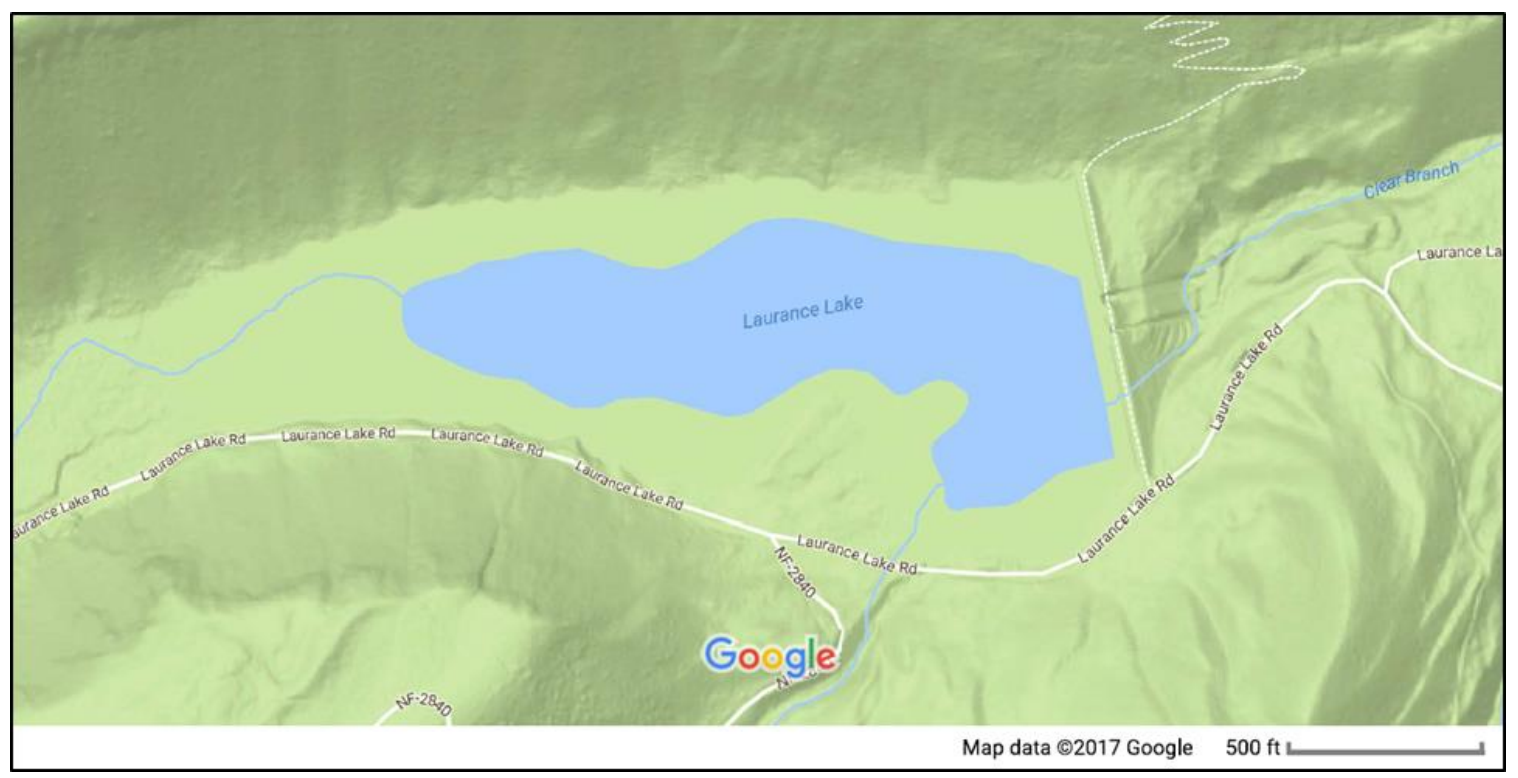

Figure 4-31. The Laurance Lake location (Google, 2017) 


\subsubsection{The 3D Model Input Bathymetry and the Grid Development}

The model input bathymetry of Laurance Lake was created from the surface contour map of the lake. Fortran90 code used with the Intel Fortran Compiler, SURFER software, and Matlab were employed together to develop a tool to extract the input bathymetry file. A horizontal grid resolution of $\Delta x=134 \mathrm{~m}$ and $\Delta y=85 \mathrm{~m}$ was used. The 3D model grid was overlaid on the contour map as shown in Figure 4-32, a top view of the 3D model grid. Vertically, $\Delta z=0.5 \mathrm{~m}$ was used as a depth increment. As a result, $\theta_{2}$ is 4.36 radians. Initially, the water surface level was at an elevation of $906.588 \mathrm{~m}$, and the initial values of water surface elevation, $\eta_{(x, y, t=0)}$, were set to zeros. Also, the surface computational grid cells were set to $k=k t=3$. 


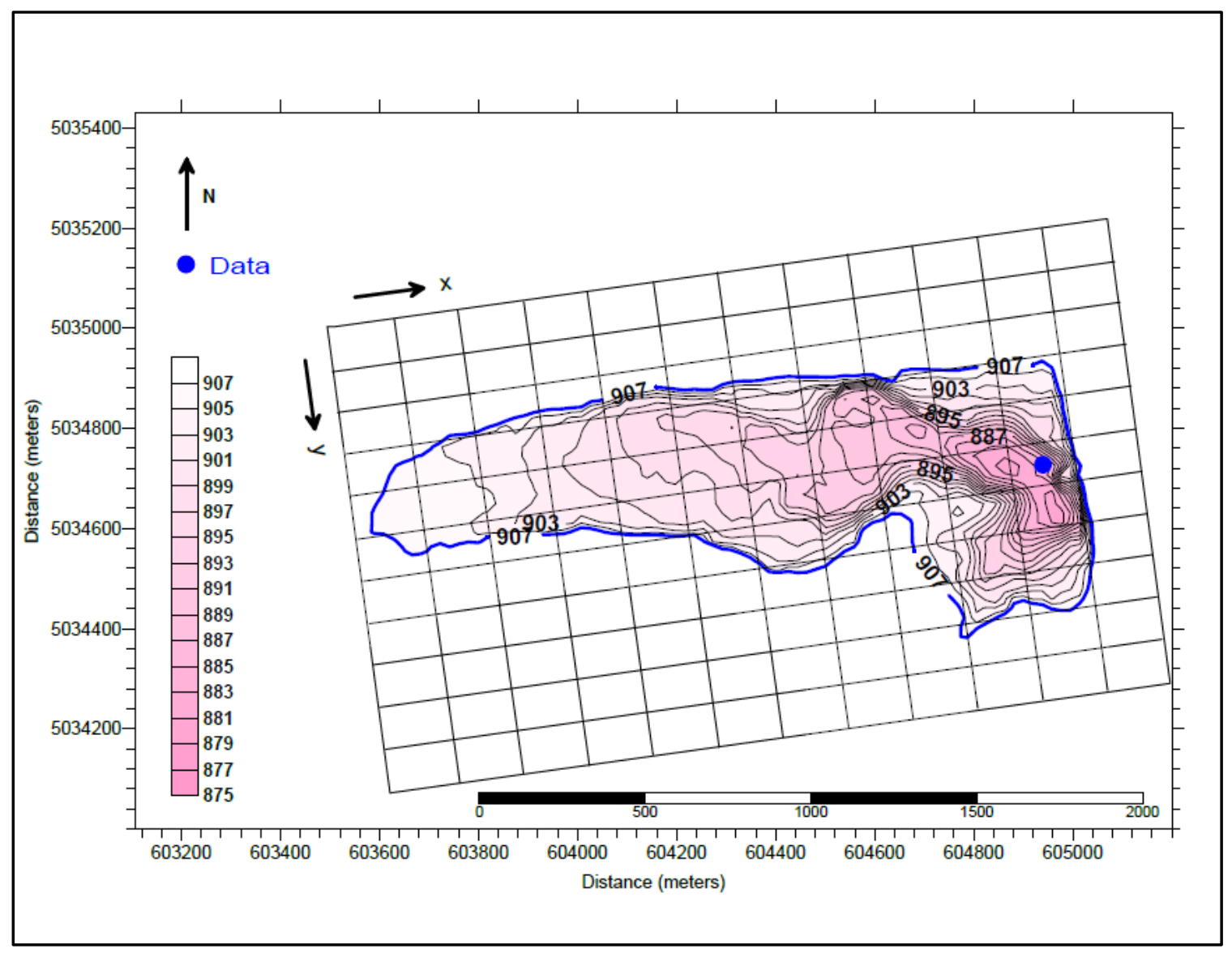

Figure 4-32. Top view of the Laurance Lake model grid

\subsubsection{The 2D CE-QUAL-W2 Model Grid Setup against the 3D Model Setup}

Using the same bathymetry and contour map, a 2D and 3D model grid were developed for each model. The 2D CE-QUAL-W2 model grid is defined by the segment length $\Delta x$ and depth $\Delta z$, while the W3 model grid is defined by the cell length $\Delta x$, width $\Delta y$, and depth $\Delta z$.

Figure 4-33 shows the grid setup of the 2D and 3D model. The 2D setup divided the lake into two branches, with each branch having its own segment length. On the other hand, 
the $3 \mathrm{D}$ setup treats the entire lake as a one system. The field data that were used for the model comparisons were collected at the specified location of the dam in the Figure 4-32.

The 3D Setup:

- $\Delta \mathrm{x}=134 \mathrm{~m}$

- $\Delta y=85 \mathrm{~m}$

- $\Delta z=0.5 \mathrm{~m}$
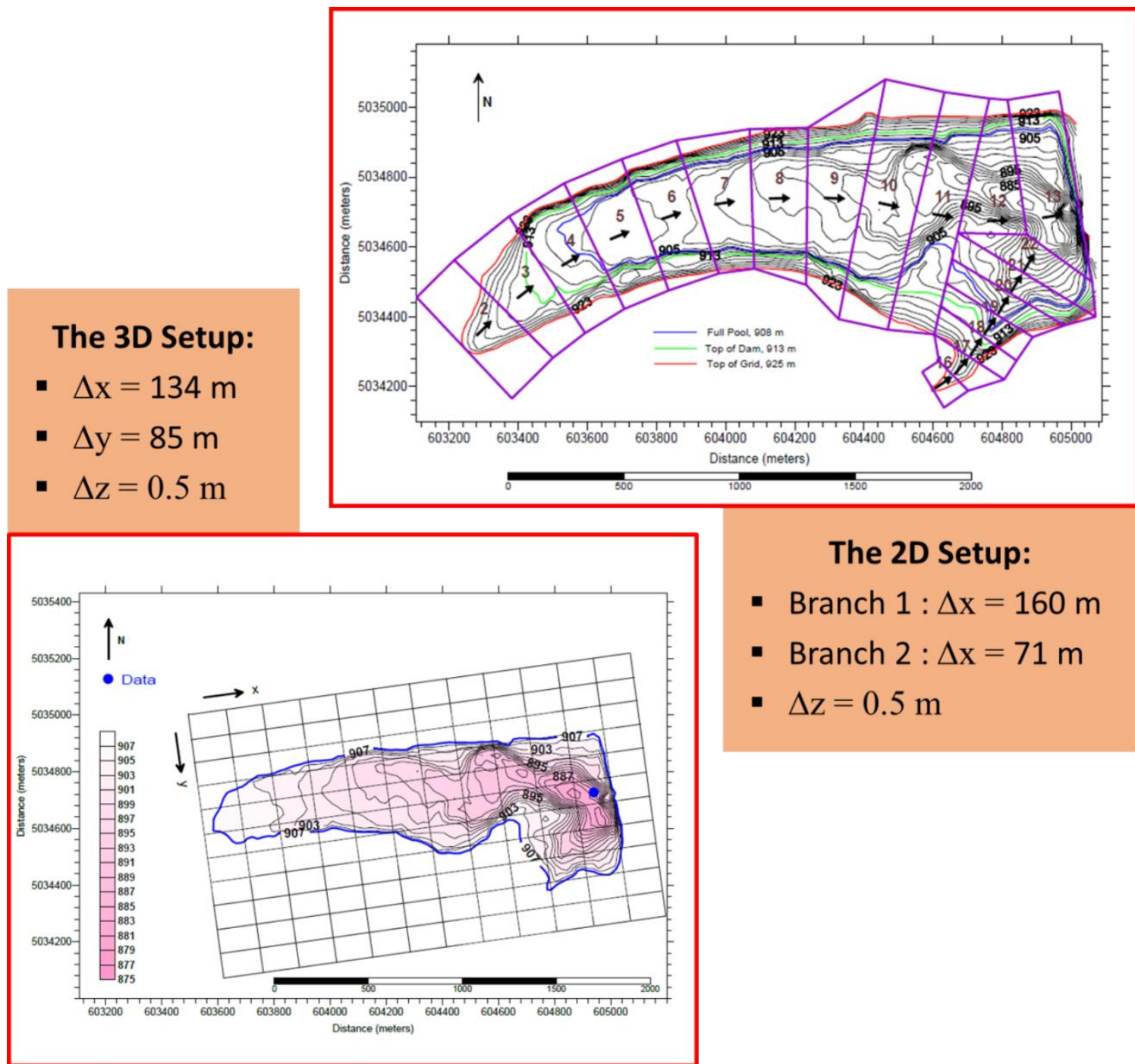

The 2D Setup:

- Branch $1: \Delta x=160 \mathrm{~m}$

- Branch $2: \Delta x=71 \mathrm{~m}$

- $\Delta z=0.5 \mathrm{~m}$

Figure 4-33. Models bathymetry and the 2D and 3D grid setup 


\subsubsection{Inflow/Outflow Boundary Conditions and Meteorological Data}

The boundary conditions required to run the Laurance Lake model were prepared based on gaging station data over the simulation period from May 1, 2003 to April 1, 2004 (Julian day: 486 - 730). Figure 4-34 and Figure 4-35 show the time series flow boundary conditions of the two inflows, Clear Branch Creek and Pinnacle Creek, respectively. The corresponding temperatures of the inflows are shown in Figure 4-36 and Figure 4-37. The outflow rate at the dam location was a withdrawal at the bottom of the dam as shown in Figure 4-38.

Figure 4-39 shows the initial temperature and u-velocity distribution with depth at the dam location. The initial temperature profile was based on the available data and the initial u-velocity profile was calculated by implementing selective withdrawal theory (Imberger \& Fischer 1970). This is an important algorithm to use at dam/reservoir withdrawals since it informs the model as to the vertical layers of the withdrawal without needing to solve the near-field dynamics with the vertical momentum equation.

The necessary meteorological data to run and calibrate the model (wind magnitude and direction, air temperature, dew point, cloud cover, and solar short radiation) were measured at the dam as shown in Figure 4-40 to Figure 4-45. For more details, see Berger et al. (2005). 


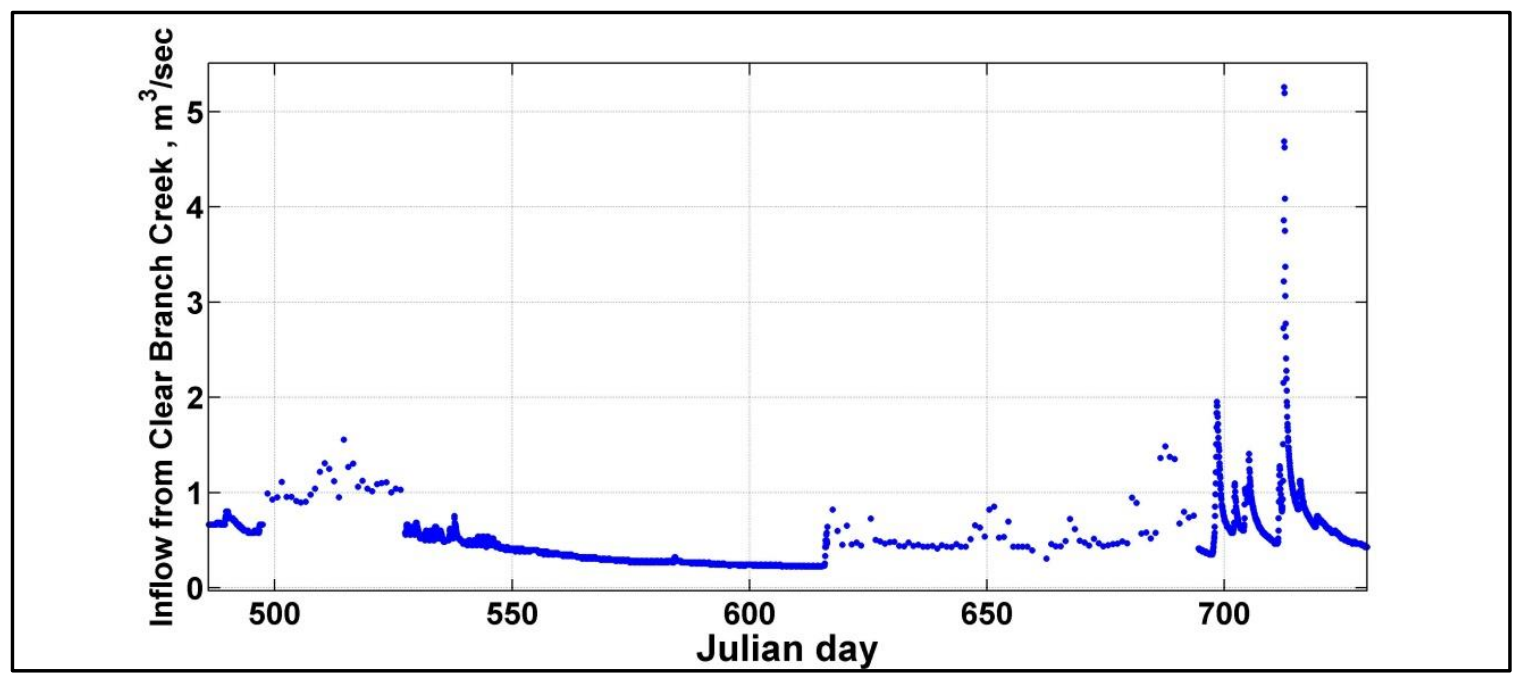

Figure 4-34. Laurance Lake inflow from Clear Branch Creek

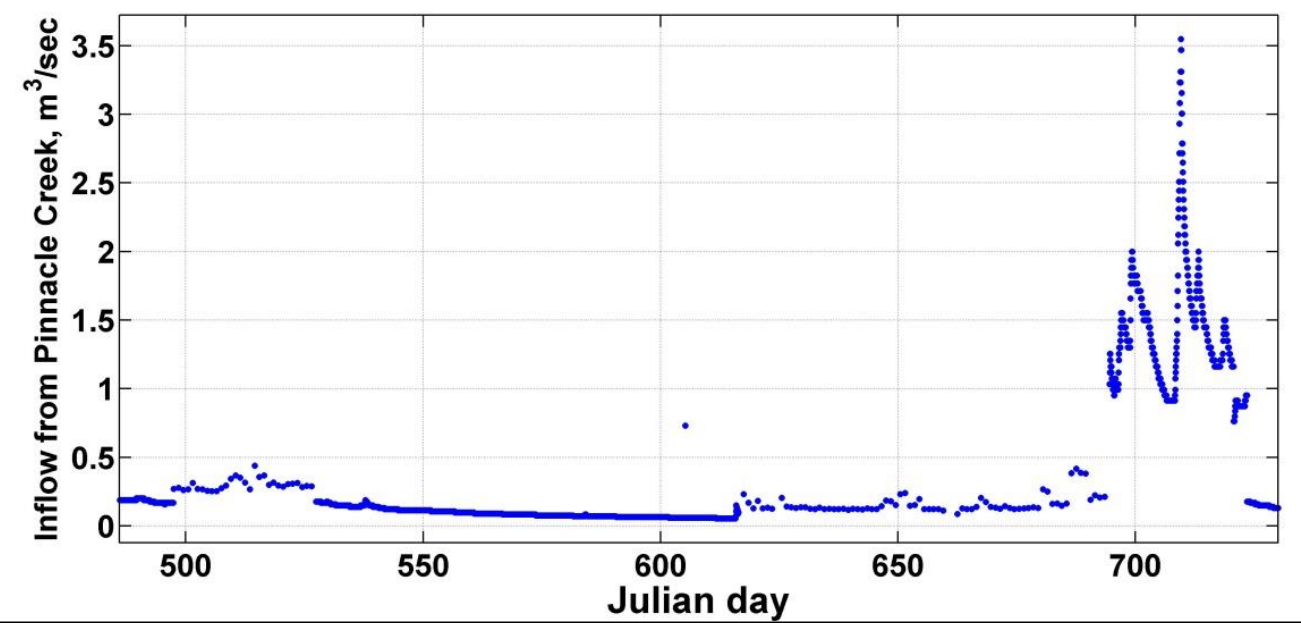

Figure 4-35. Laurance Lake inflow from Pinnacle Creek 


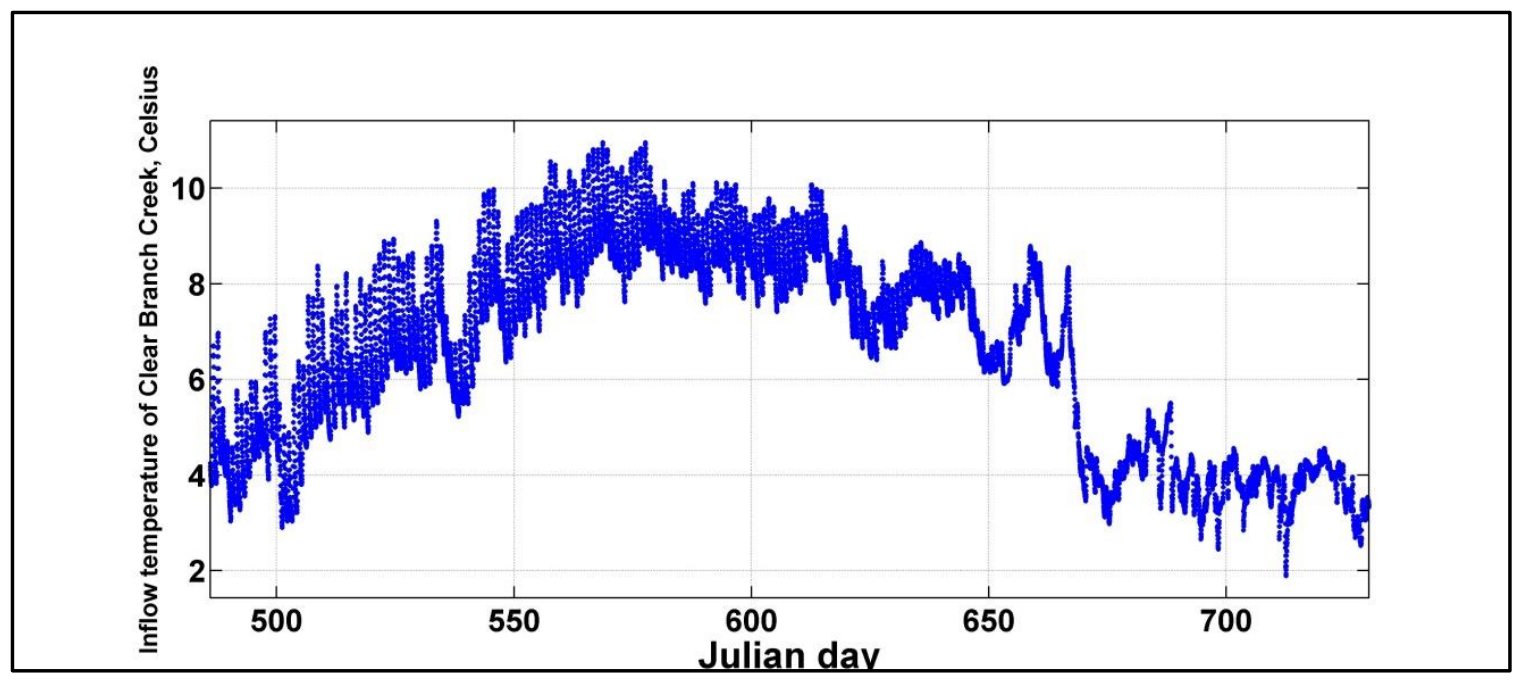

Figure 4-36. Laurance Lake inflow temperature of Clear Branch Creek

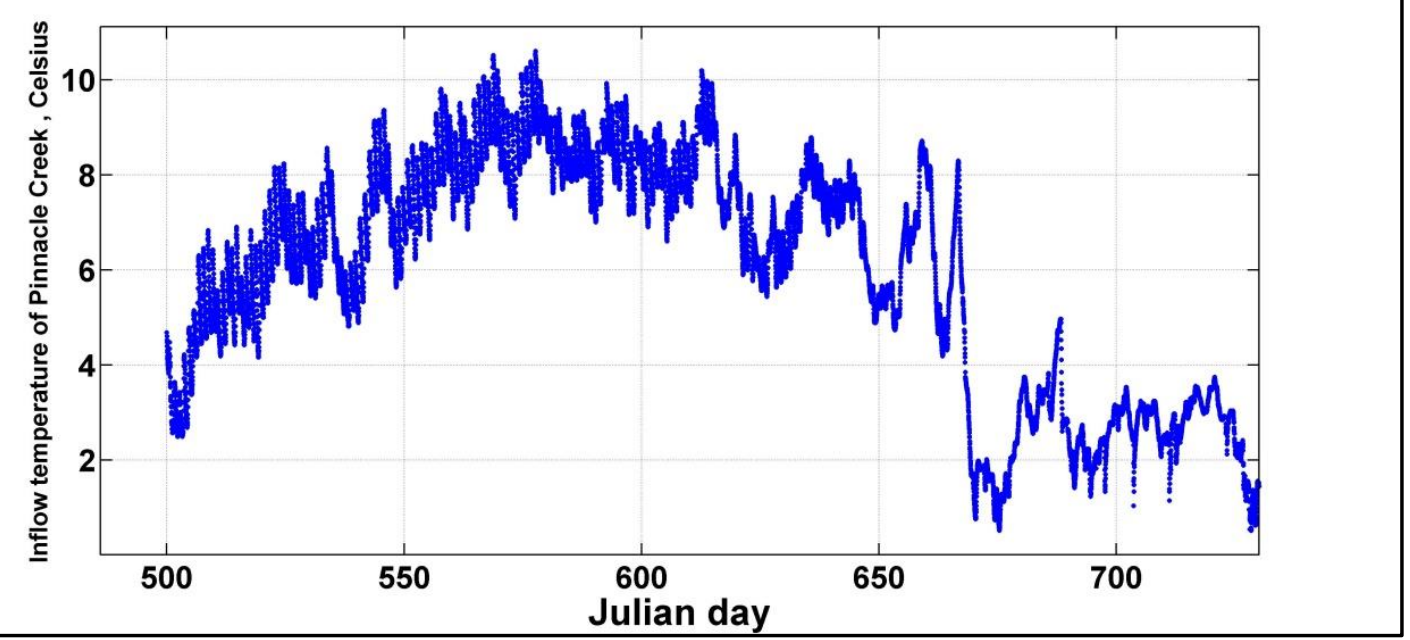

Figure 4-37. Laurance Lake inflow temperature of Pinnacle Creek 


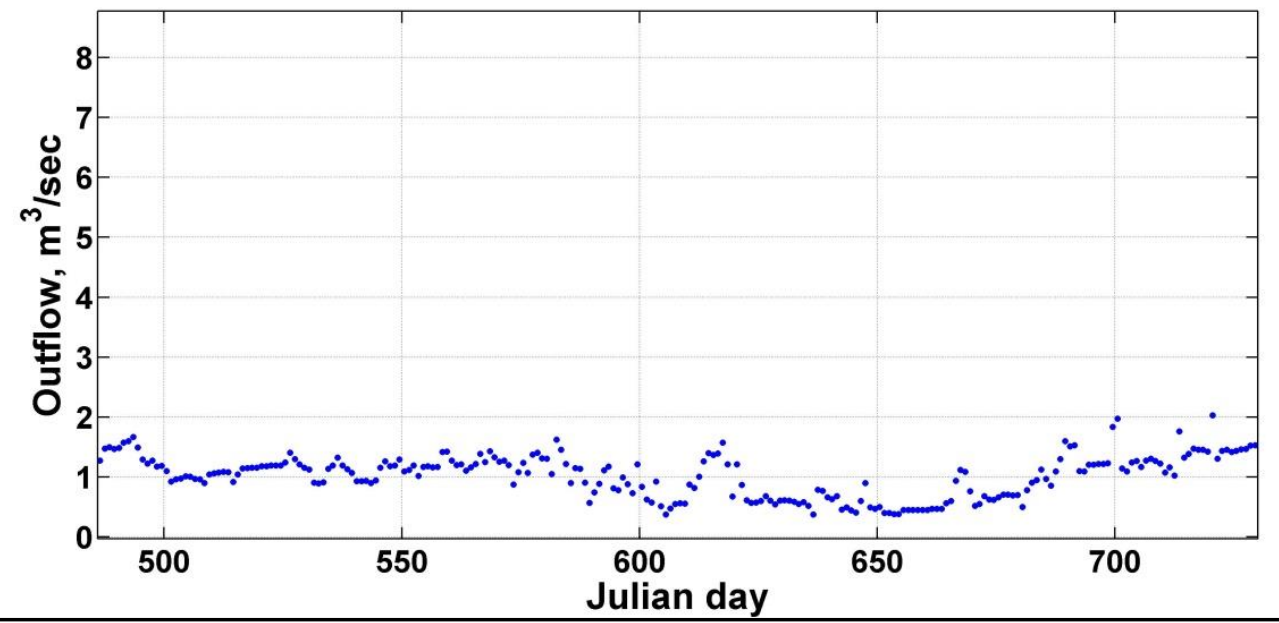

Figure 4-38. Laurance Lake outflow to Clear Branch Creek

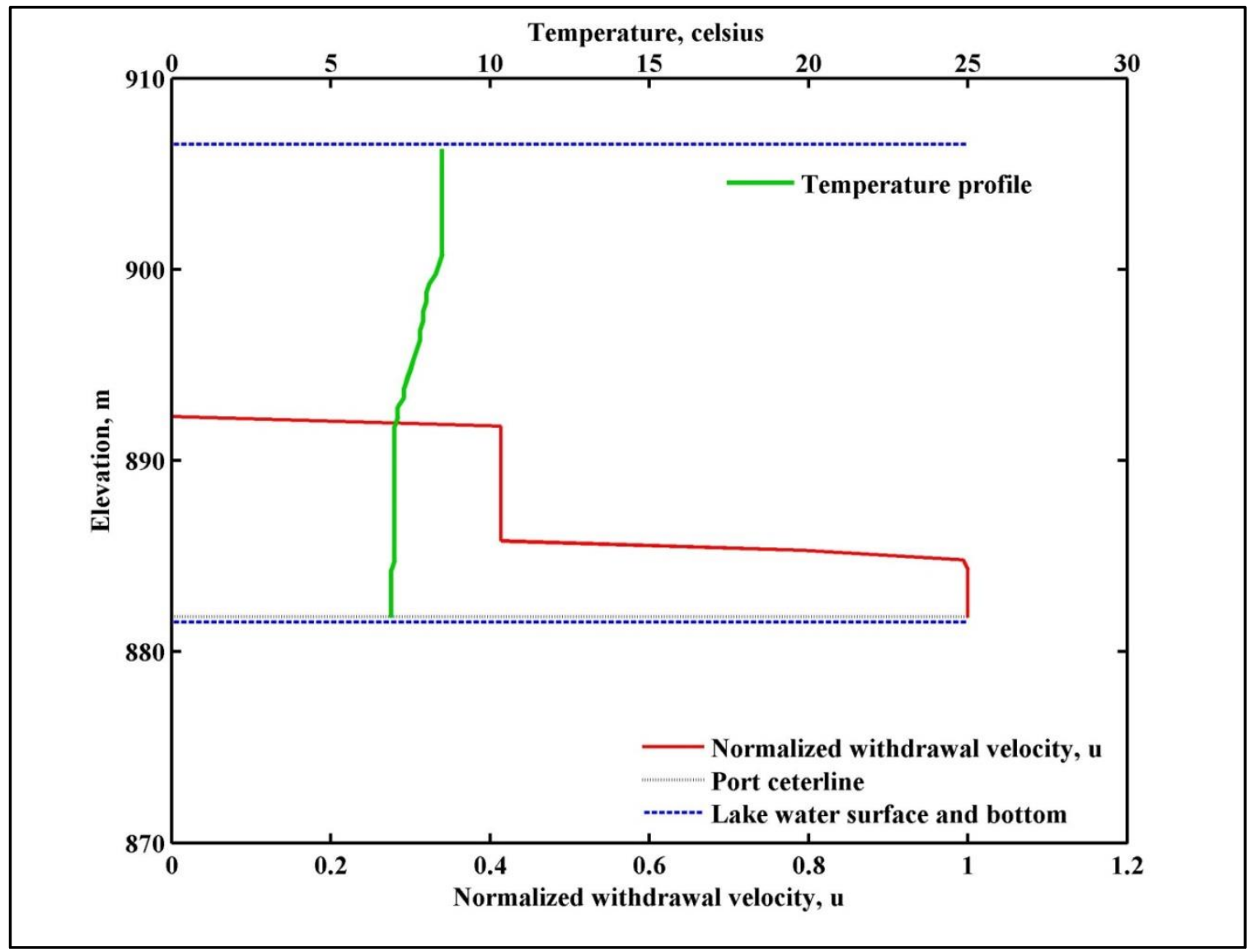

Figure 4-39. Initial temperature profile and normalized u-velocity at the dam 


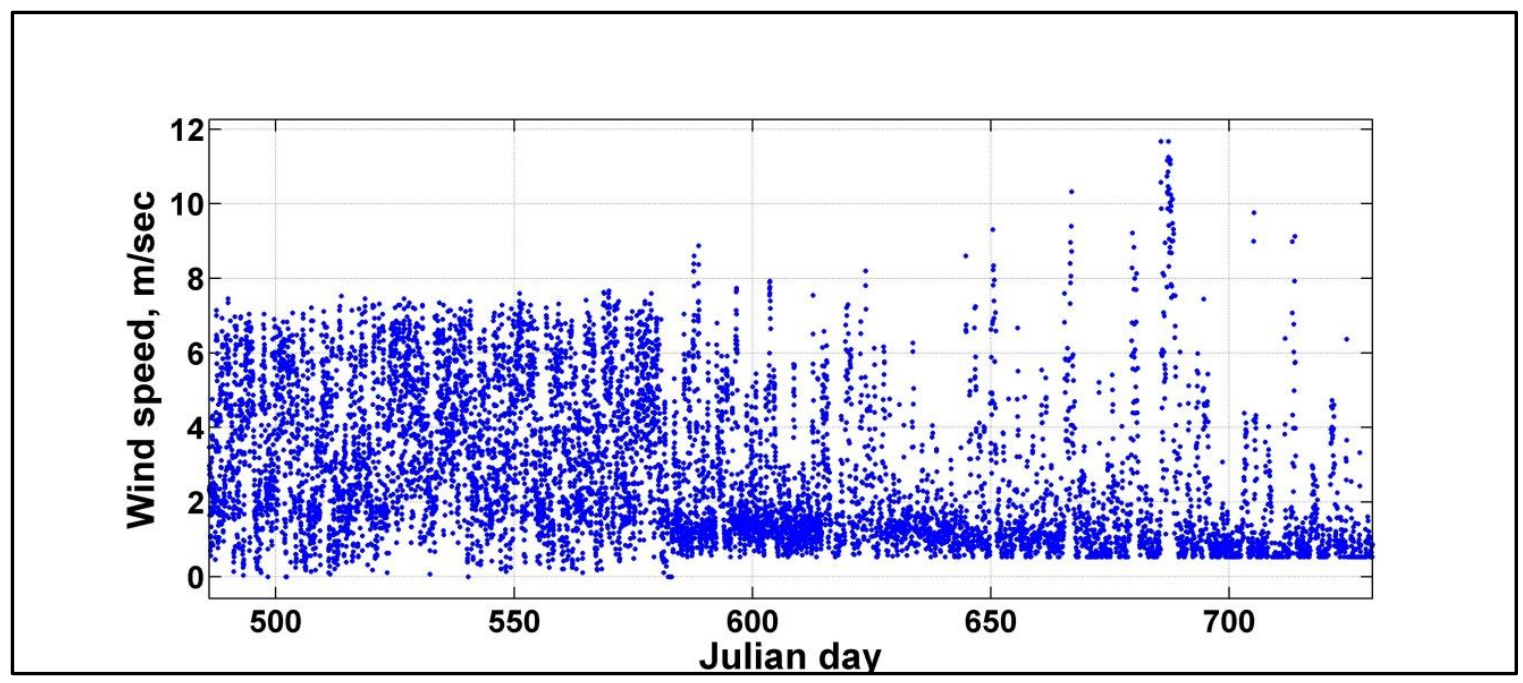

Figure 4-40. Wind speed input data of the Laurance Lake model

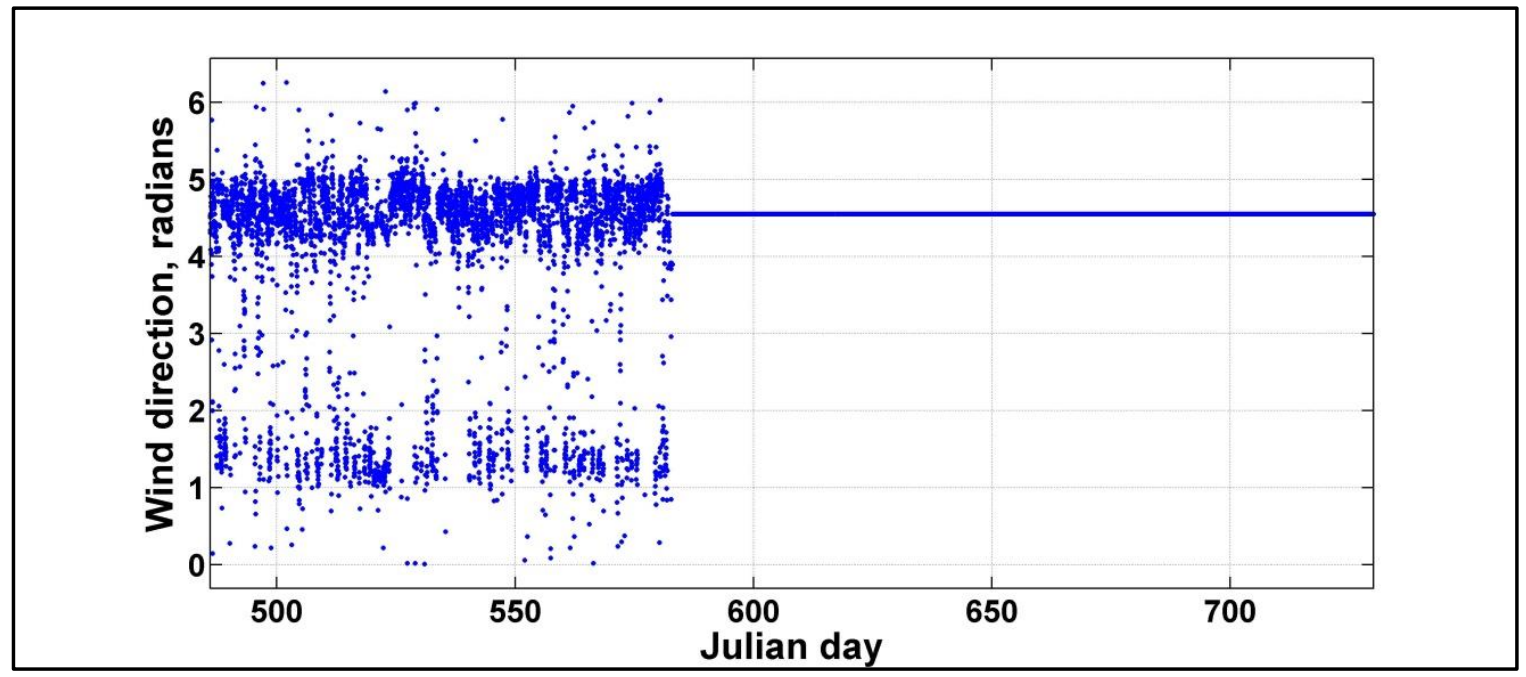

Figure 4-41. Wind direction input data of the Laurance Lake model 


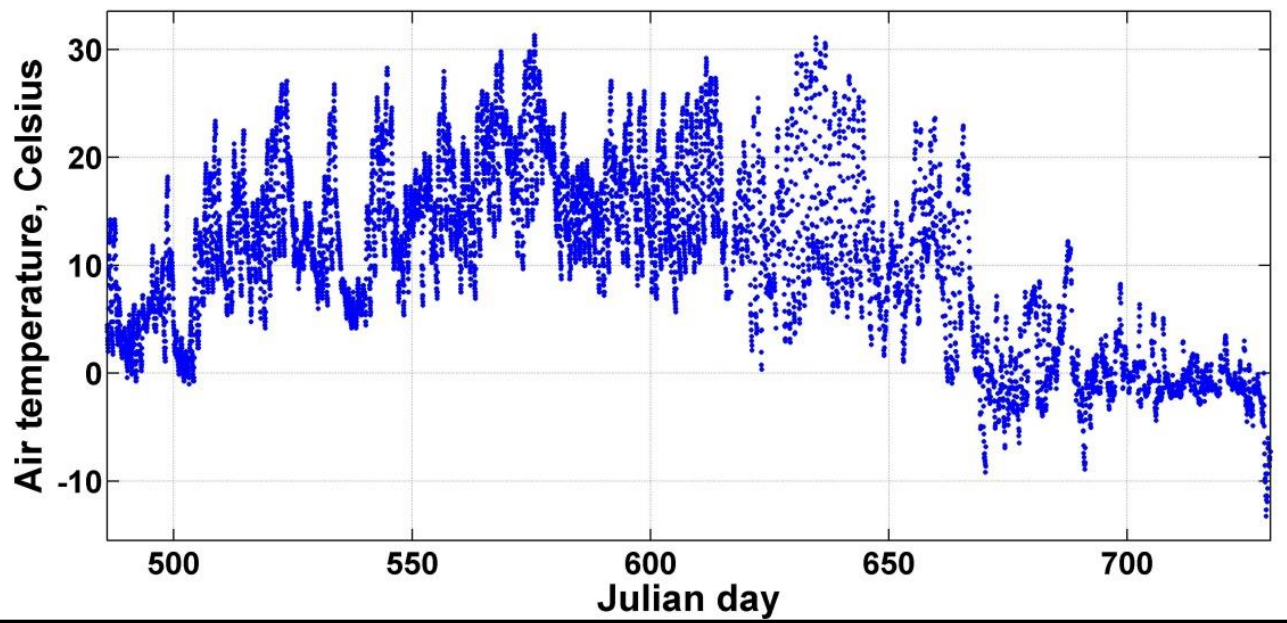

Figure 4-42. Air temperature input data of the Laurance Lake model

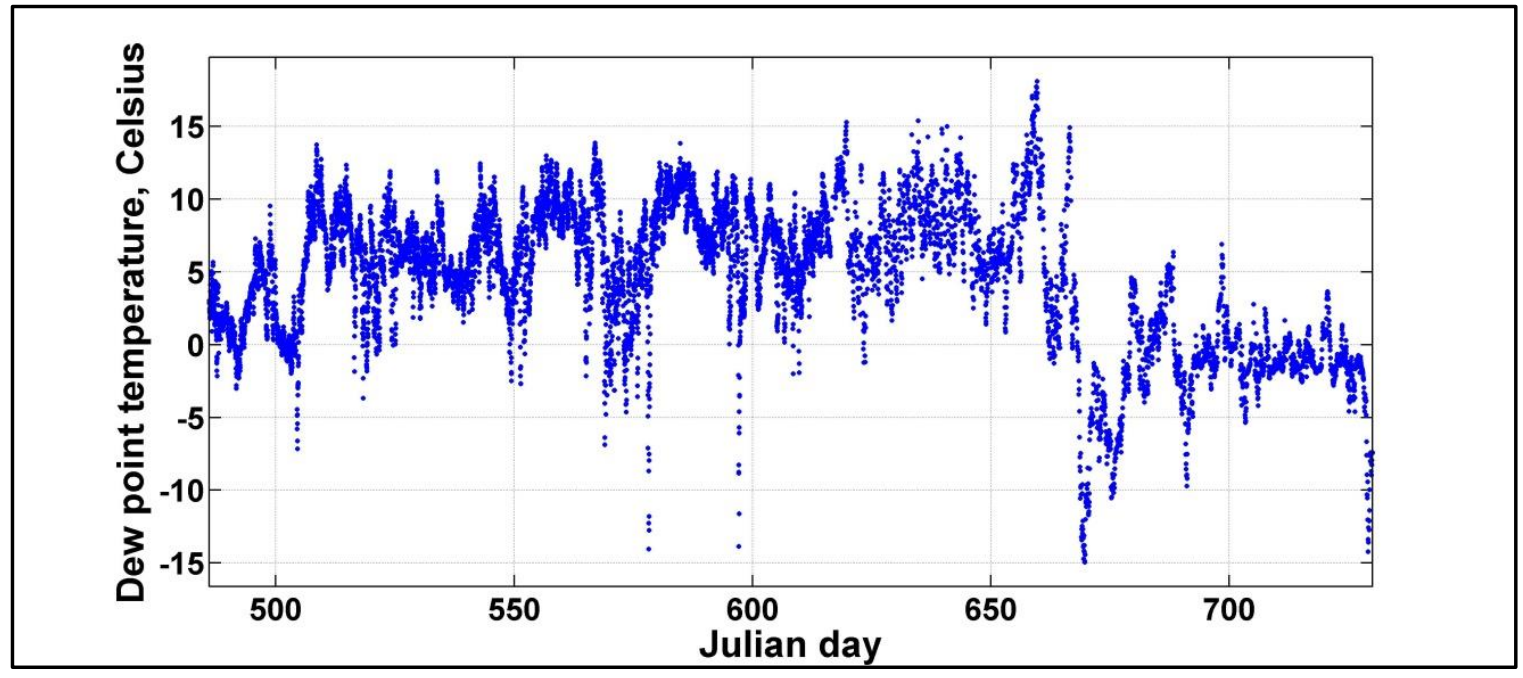

Figure 4-43. Dew point temperature input data of the Laurance Lake model 


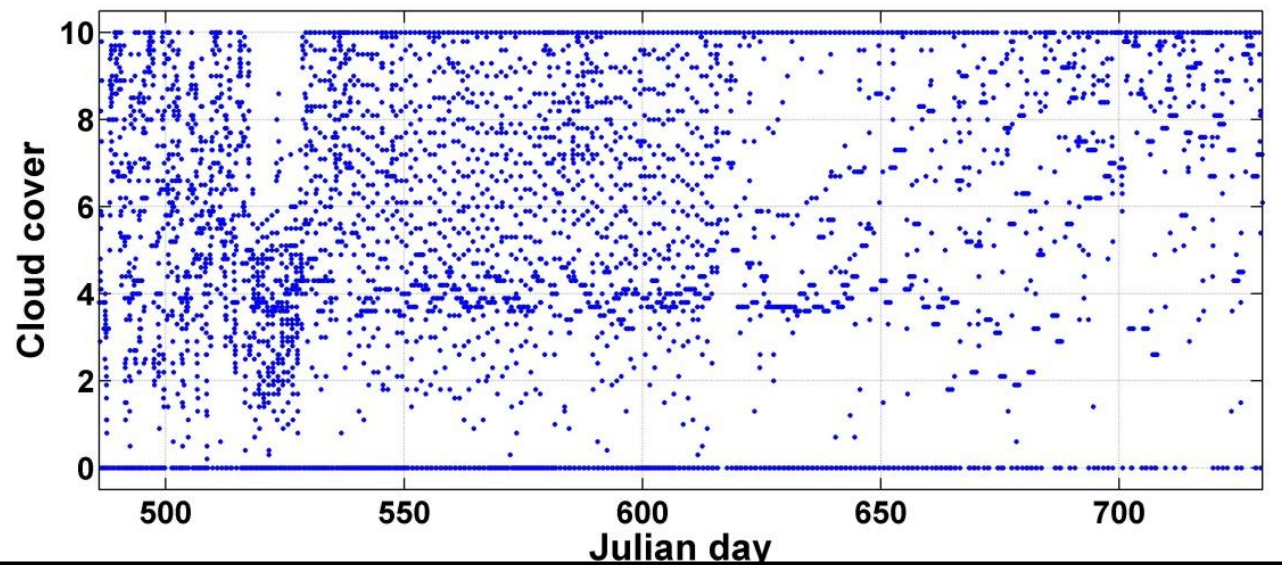

Figure 4-44. Cloud cover input data of the Laurance Lake model

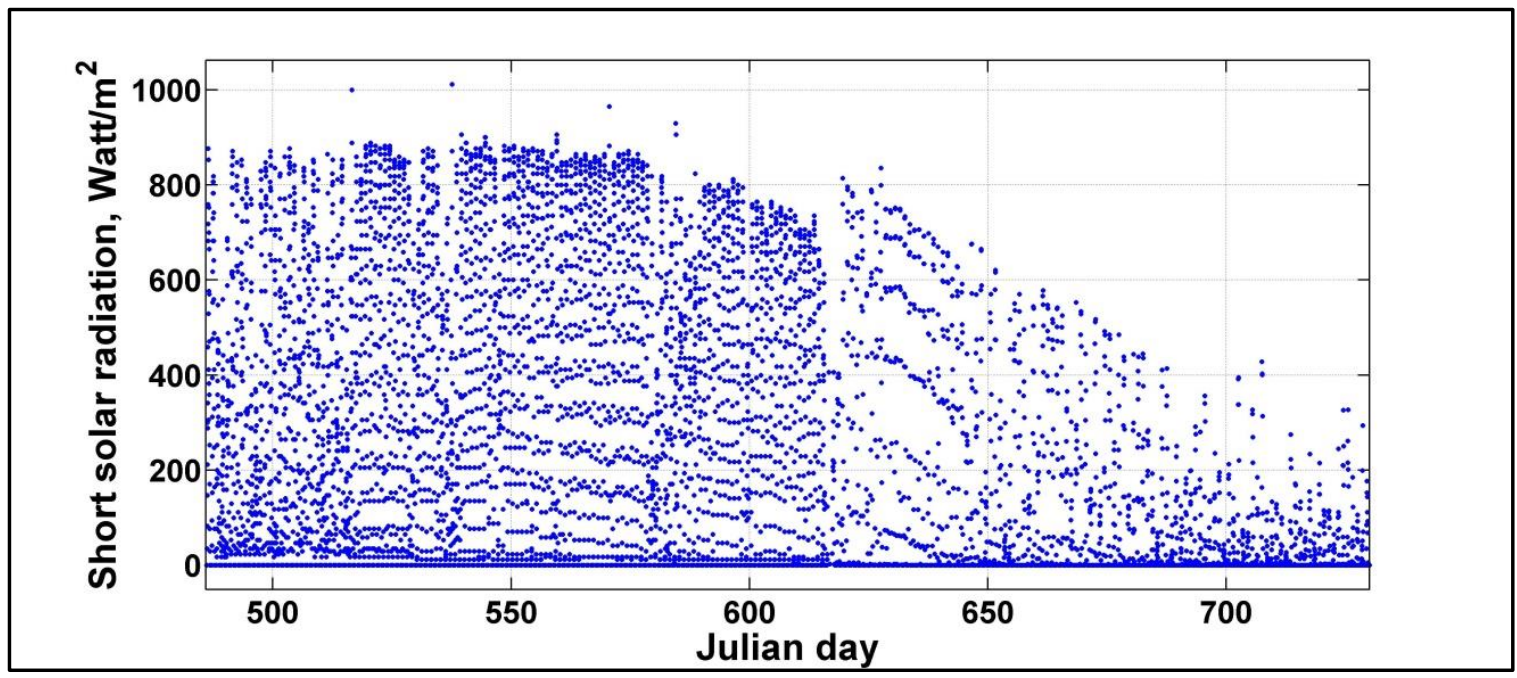

Figure 4-45. Short solar radiation input data of the Laurance Lake model 


\subsubsection{Comparison between the 2D and 3D Model for the Lake System}

The 3D model was calibrated over the simulation period by using a time step of $3 \mathrm{sec}$ or less, whereas the 2D CE-QUAL-W2 model runs used a maximum time step as high as $3600 \mathrm{sec}$ and average time step of $156 \mathrm{sec}$. Both models were run on the same PC (Windows 10, Intel(R) Core(TM) i7-5500U CPU @ 2.40 GHz, installed memory (RAM) 8.00 GB, 64-bit Operating system X64-based processor). The 3D model took $66 \mathrm{~min}$ actual time (CPU time: $59.6 \mathrm{~min}$ ) to execute, while the 2D CE-QUAL-W2 model took 59 sec (CPU time: $0.96 \mathrm{~min})$. This was the main difference between the $2 \mathrm{D}$ and the $3 \mathrm{D}$ model, making the 3D computational time cost much more "expensive" by a factor of about 60 .

Figure 4-46 shows the 2D and 3D model predictions of water level compared with field data at the dam location. Error statistics between model predictions and water level data showed that the models were in good agreement with field data. However, model error was higher for the $3 \mathrm{D}$ model compared to the $2 \mathrm{D}$ model. Because the $2 \mathrm{D}$ predictions are considered uniform across the segment width and the side flow regime is different from the central flow, the 2D model cannot show water level variability laterally. Therefore, the location of the dam outflows could be a factor affecting the local water level. However, it was found that the 3D water level predictions were almost the same laterally. 


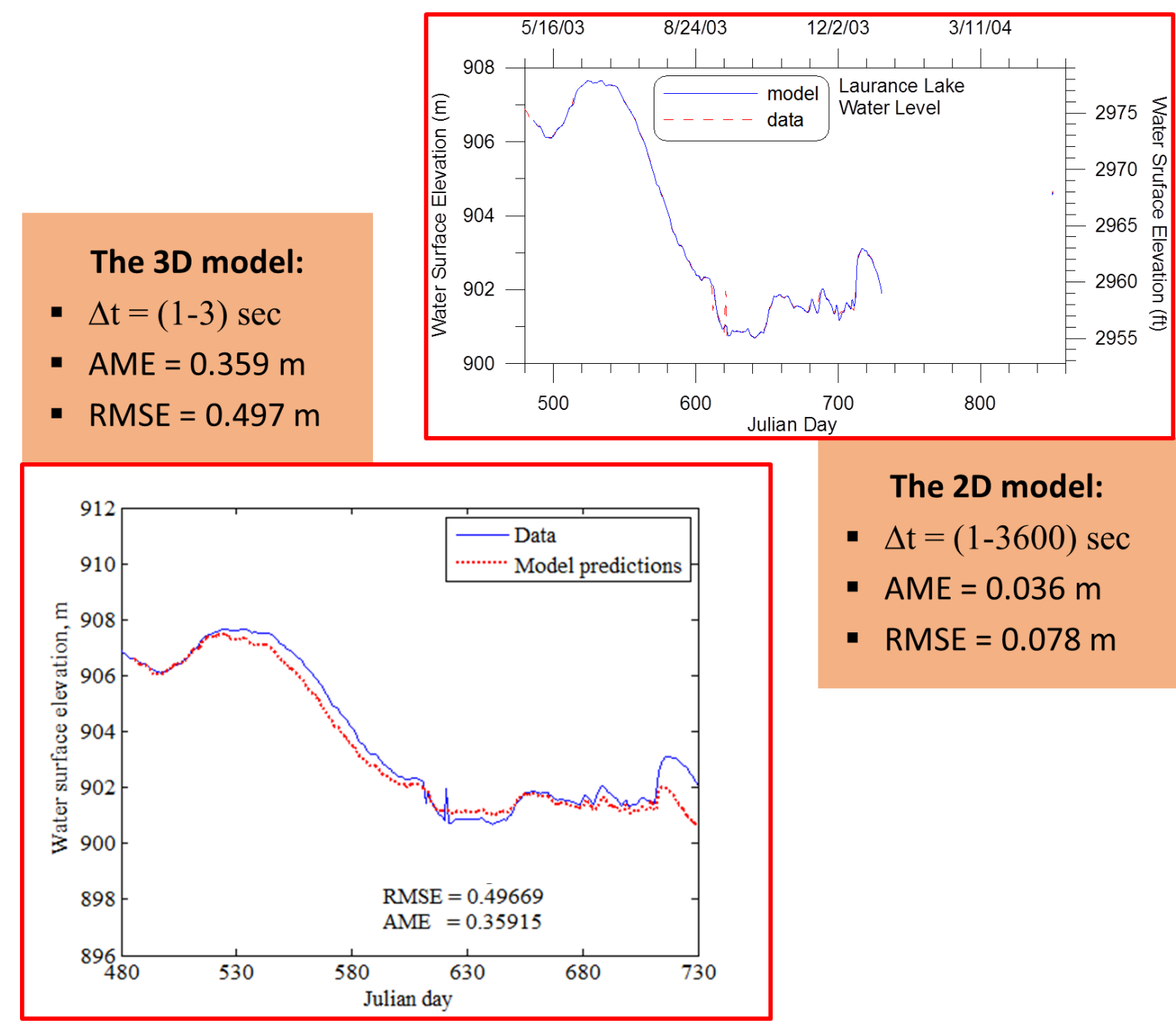

Figure 4-46. The 2D and 3D model predictions of water level compared with field data 
Figure 4-47 shows the variable extinction coefficient values over the calibration period based on field data measurements. The 2D and 3D model predictions of temperature were calibrated over the simulation period based on the model parameters that control heat transfer across the water surface (extinction coefficient, evaporation wind speed function, percent of light absorbed at the water surface, and wind-sheltering coefficient). These parameters were calibrated differently between the $2 \mathrm{D}$ and the $3 \mathrm{D}$ model. It was found that it was not necessary to calibrate a model based on the same values that worked with the other model.

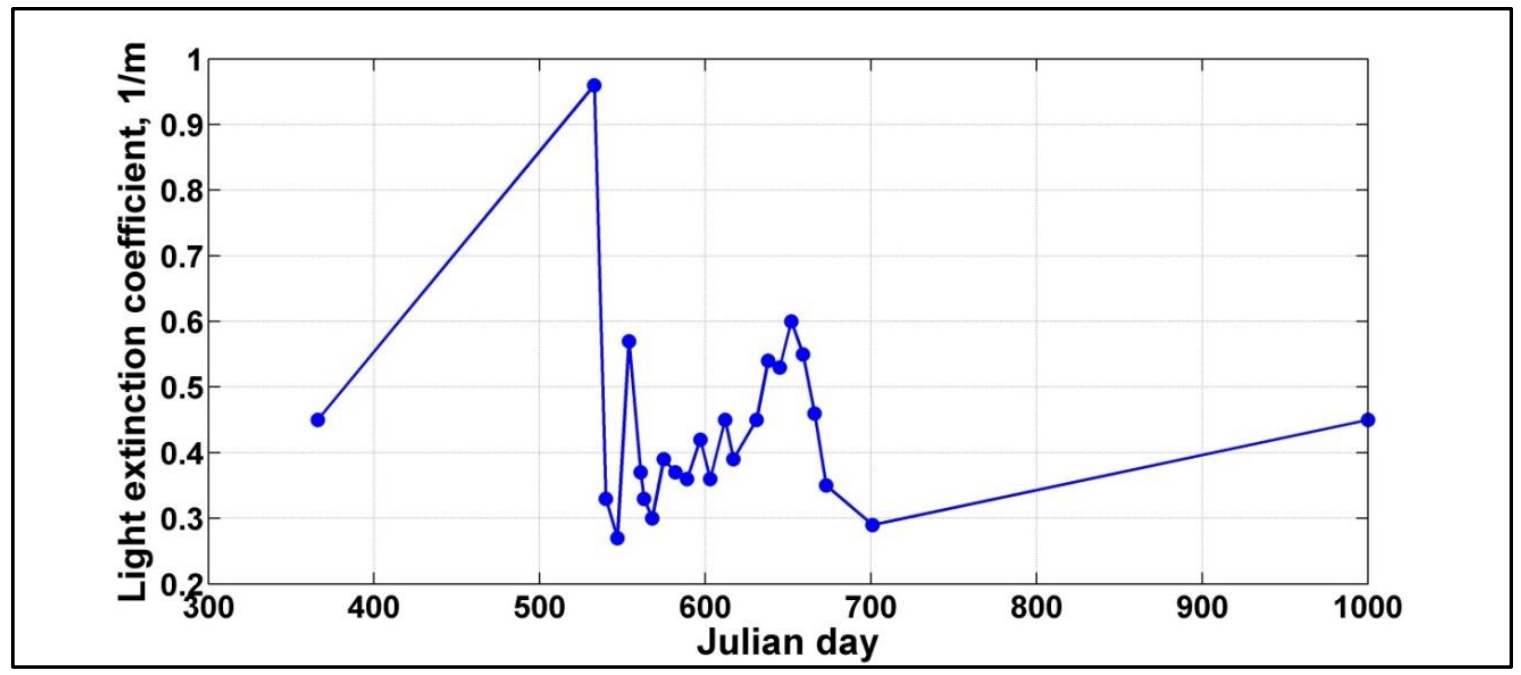

Figure 4-47. Extinction coefficient values based on Secchi disk data

The 2D and 3D model predictions of temperature were compared with field data at various times during the simulation period. Figure 4-48 and Figure 4-49 show the 2D and 3D model predictions of vertical temperature profiles compared with field data. In spite of the good agreement with field data for both models, the 3D W3 model has a higher 
absolute mean model error (by approximately $0.06^{\circ} \mathrm{C}$ ). In addition, the calibration process for the 3D model was much slower than the 2D model as a result of the slower computational speed of the 3D model. Also, Figure 4-50 shows the model predictions of temperature profiles after adding the ULTIMATE QUICKEST scheme to the code.

Another main difference between the 2D and 3D model is the velocity field representation. The 2D model can simulate the flow path in the longitudinal-vertical direction. This $2 \mathrm{D}$ representation is applicable for lakes that are typically narrow; otherwise, the domain must be divided into branches of different flow paths as shown in the 2D setup in Figure 4-33. The 2D setup makes the problem difficult if the lake is wide and has irregular physical boundaries. For such a case, the 3D model works better because the lake physical domain is already represented without dividing the model into branches as shown in Figure 4-51, which is an example of the surface velocity field generated by the 3D W3 model. Therefore, the 2D model simulates the laterally averaged longitudinal velocity. A comparison between the longitudinal velocity along the 3D physical domain center-line and the laterally averaged longitudinal velocity of the 2D model was performed as shown in Figure 4-52. The longitudinal velocity predictions of the 2D model were less than those of the 3D Model. The 2D model hence predicts lower velocity and a longer travel time along the center-line. As expected, the comparison showed that both 2D and 3D longitudinal velocities have the same order of magnitude even though the 3D centerline velocity is higher. 


\section{The 2D model : Overall $\mathrm{AME}=0.429^{\circ} \mathrm{C}$}

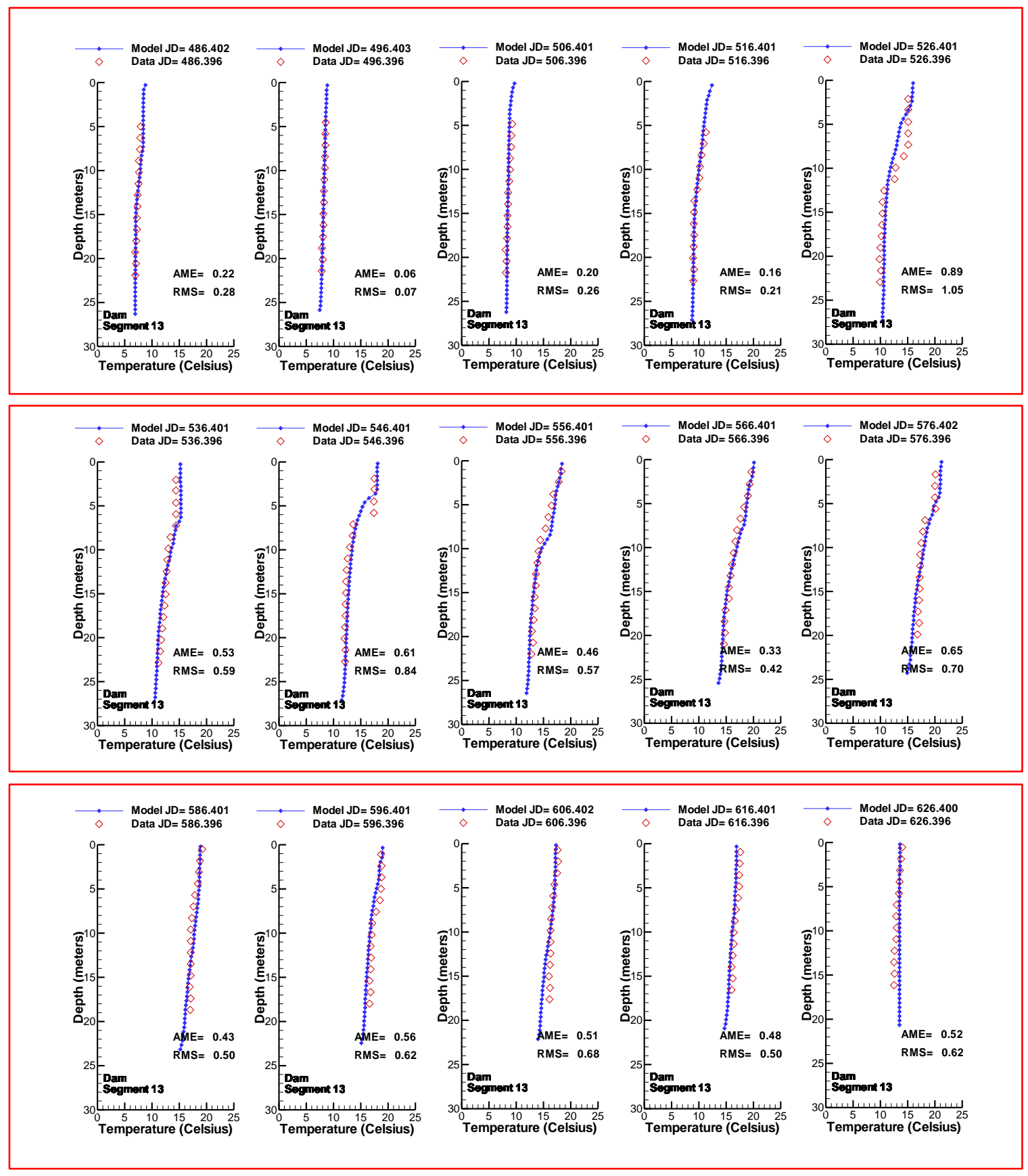




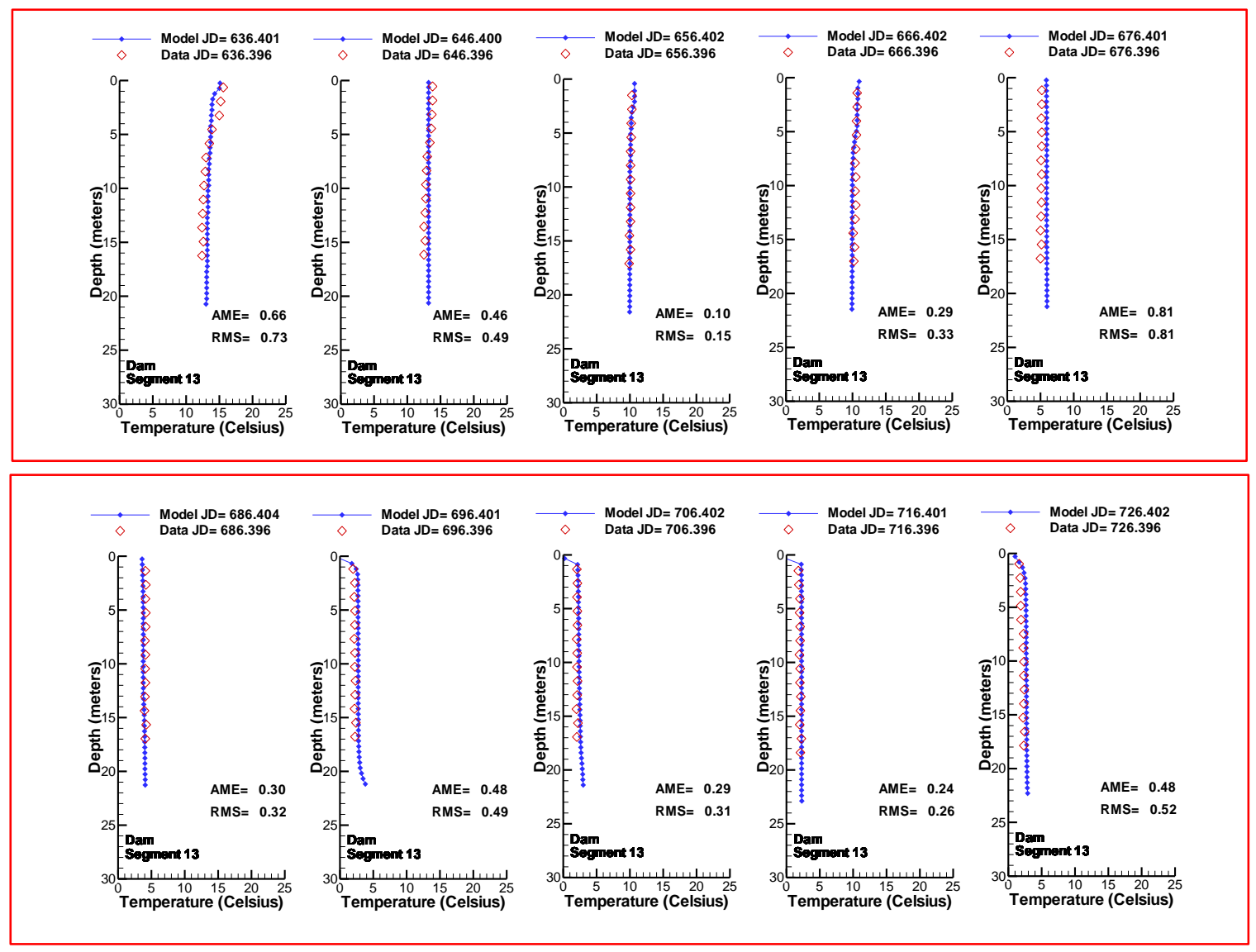

Figure 4-48. The 2D model predictions of vertical temperature profiles compared with field data 


\section{The 3D model : Overall $\mathrm{AME}=0.486{ }^{\circ} \mathrm{C}$}
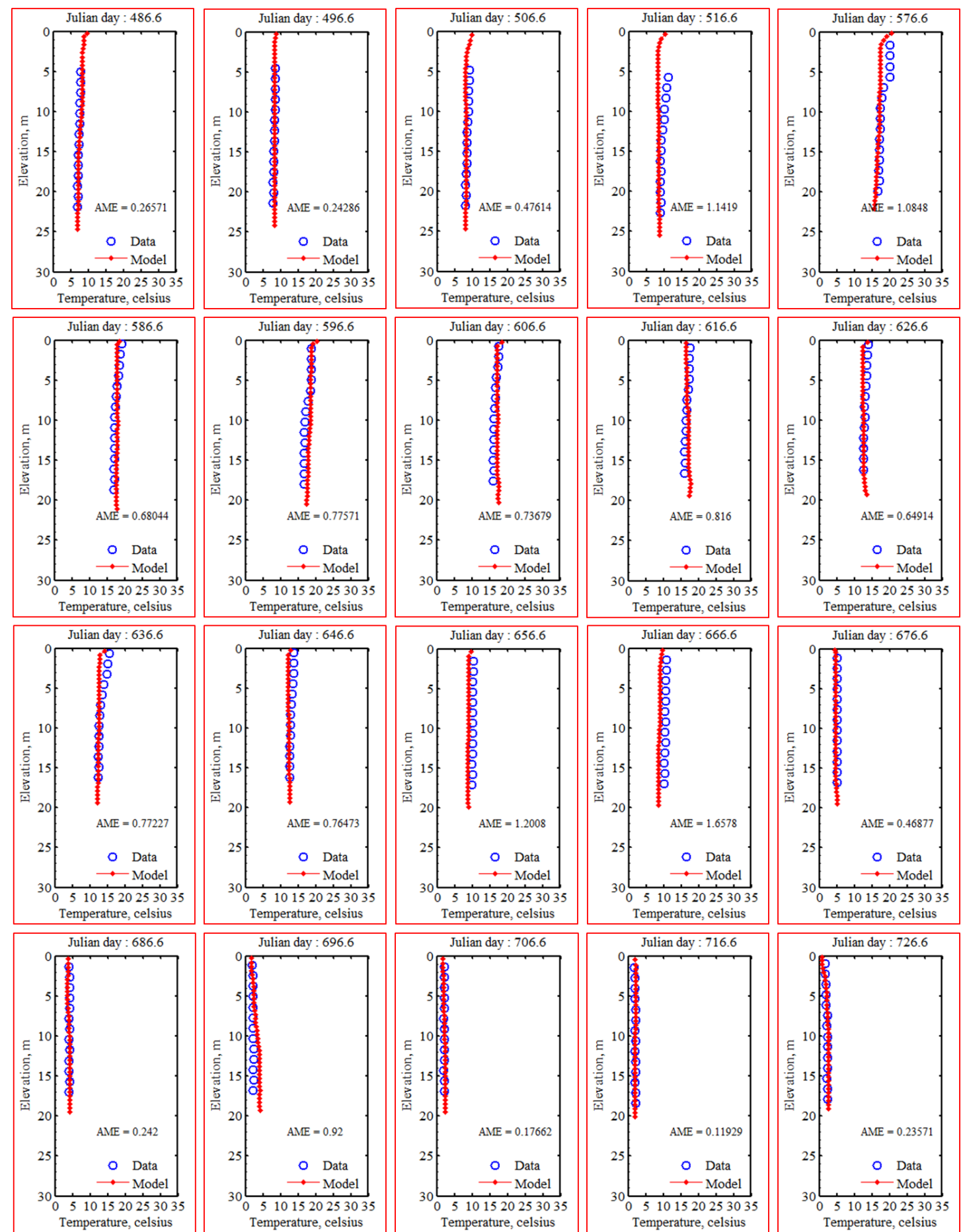

Figure 4-49. The 3D model predictions of vertical temperature profiles compared with field data using the QUICKEST scheme 

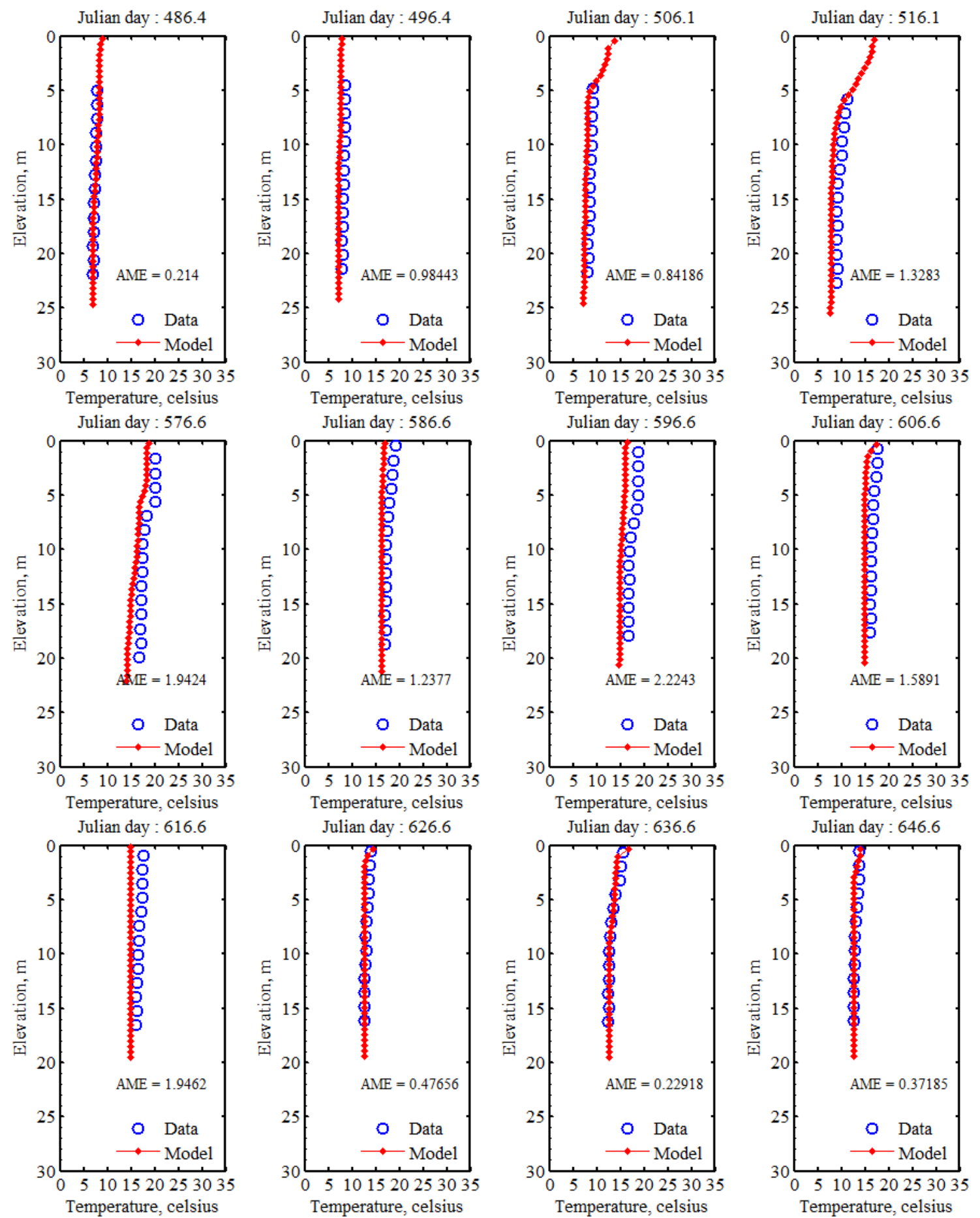

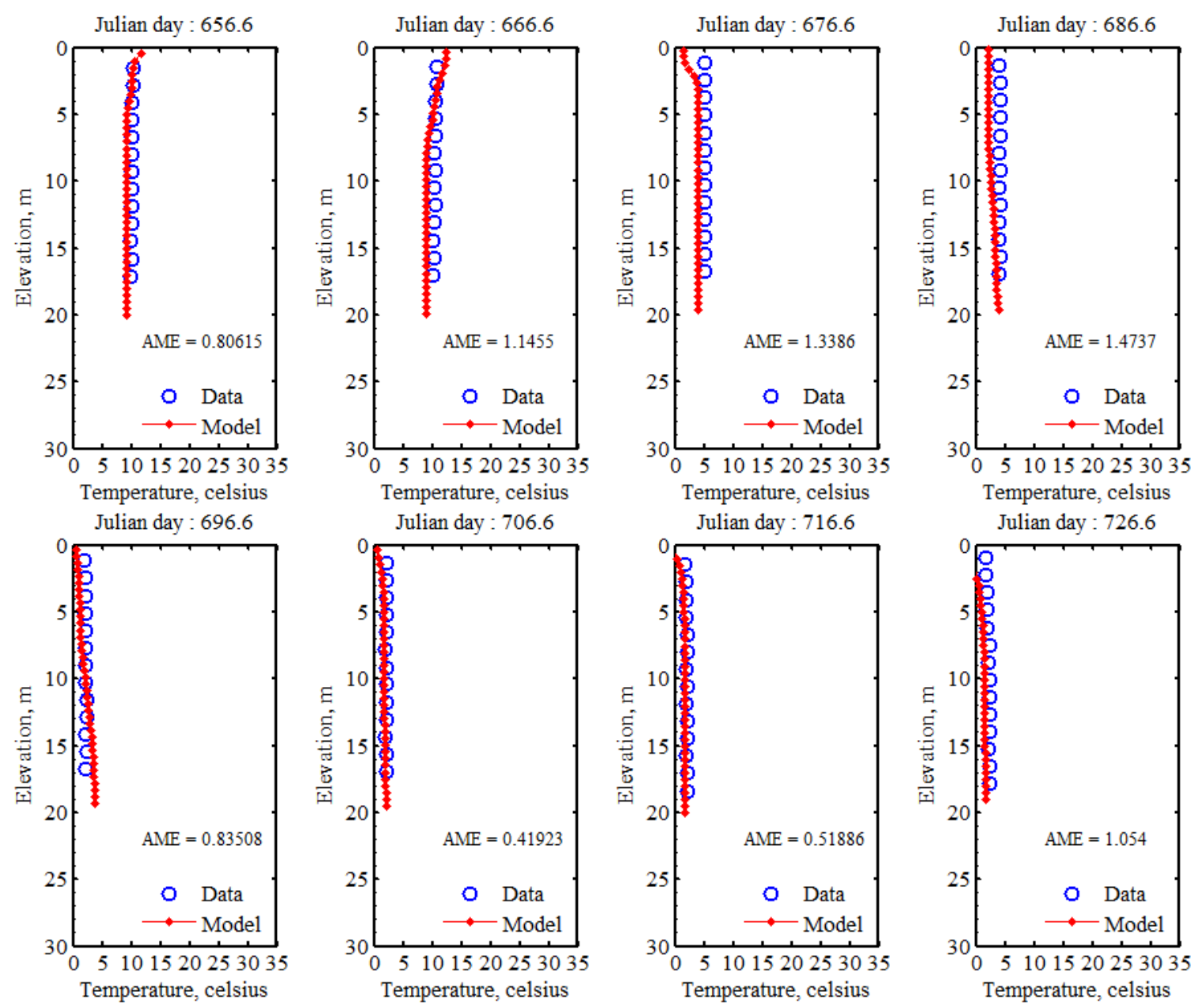

Figure 4-50. The 3D model predictions of vertical temperature profiles compared with field data using the ULTIMATE QUICKEST scheme 


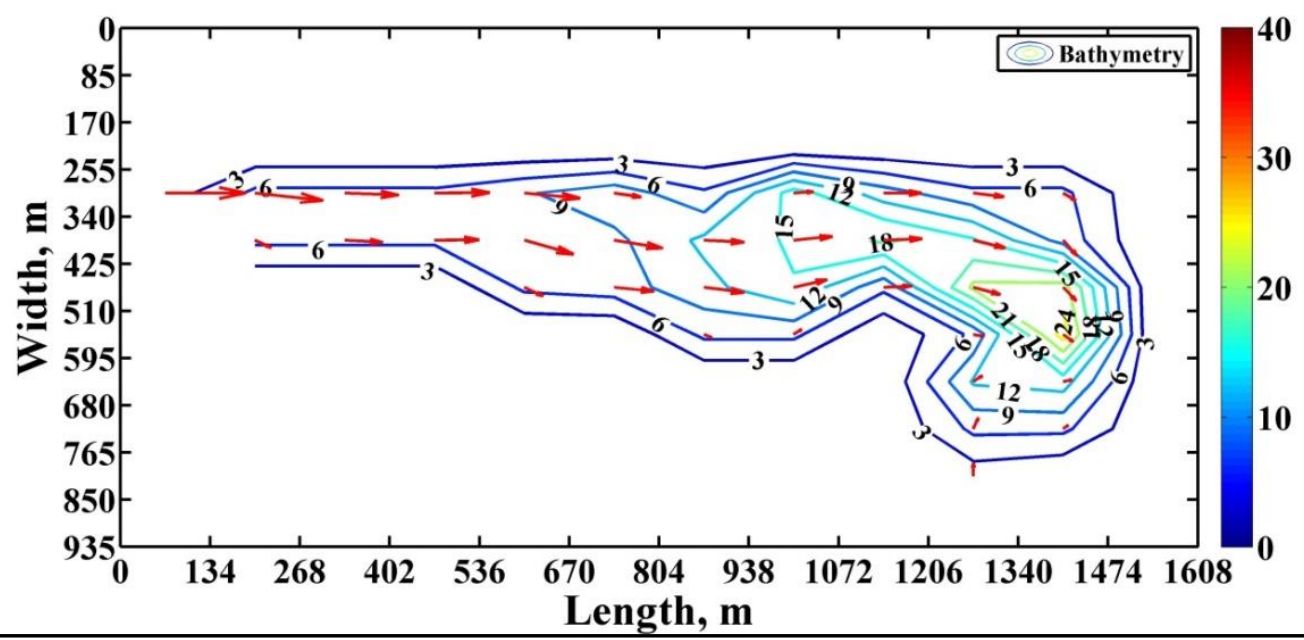

Figure 4-51. Surface velocity field of the 3D W3 model

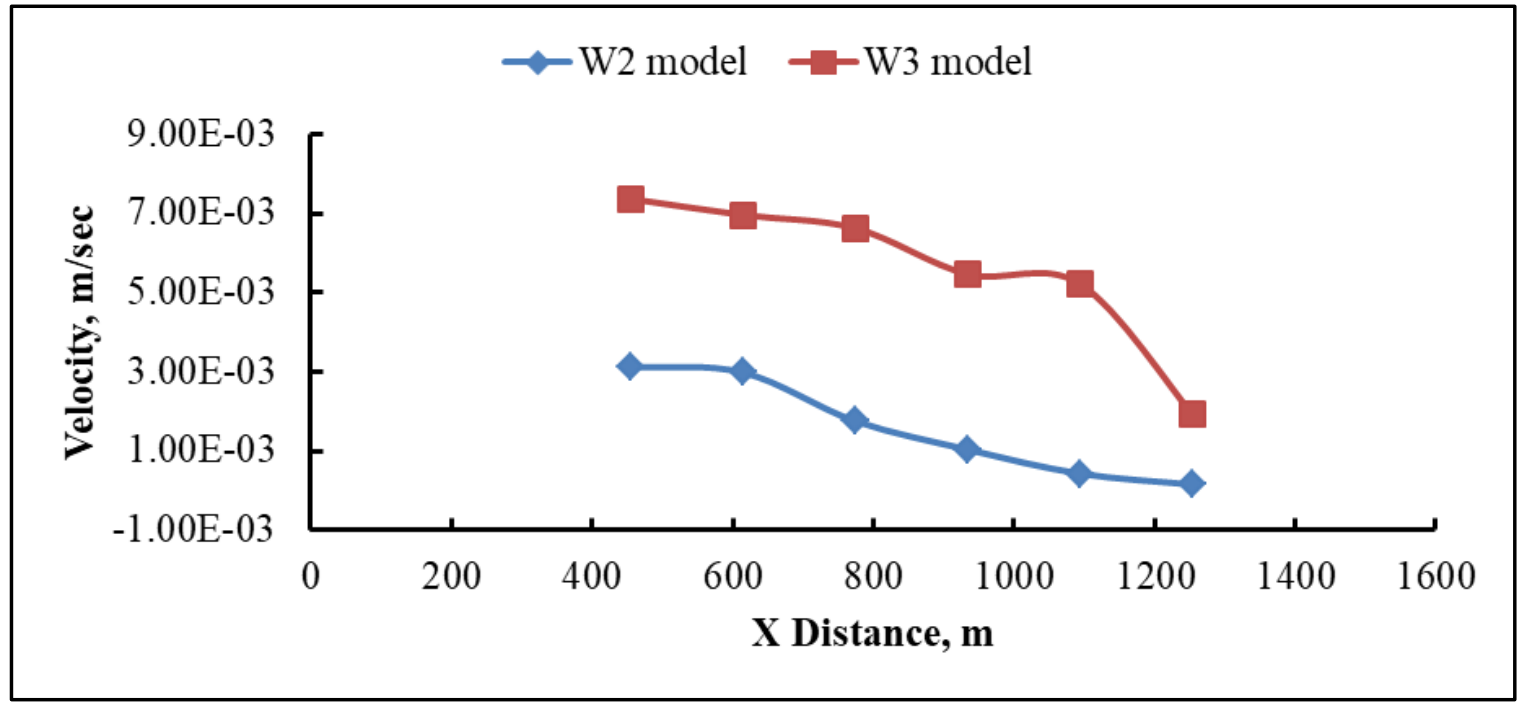

Figure 4-52. The 2D and 3D numerical predictions of longitudinal velocities at the lake longitudinal center-line at the end of the simulation time 
In summary, a 2D and 3D numerical model were applied to simulate hydrodynamics and temperature dynamics in Laurance Lake. The numerical scheme of the 3D W3 model was based a similar scheme in the 2D CE-QUAL-W2 model. The predictions of both models were compared with field data. The comparison error statistics of water level and temperature showed that both models were in good agreement with field data. However, the simulation time for the 3D model was much longer than the 2D CE-QUAL-W2 (approximately 60 times longer).

The 3D simulation time though was very dependent on the grid resolution. In this case, the 3D model had a finer horizontal grid resolution than the 2D model but they had similar vertical resolutions. The results indicated that the $2 \mathrm{D}$ model predictions were somewhat more accurate than the 3D model. Also, the grid development of the 2D model could be considered somewhat more difficult than the 3D model since the 2D model setup required a dividing of the physical domain into branches of different flow paths and increments. From this analysis, the 2D model was adequate to represent the main longitudinal-vertical variations in water level and temperature, and it has the significant computational advantage compared to the 3D model. The 3D model though can more accurately assess lateral velocity field variations if these are important to assess. 


\subsubsection{Link between the Hydrodynamics and Water Quality}

One of the main reasons why the present three-dimensional model is unique is that it solves the water quality at the same time step as the hydrodynamics. Therefore, to show the impact of this link between water quality and hydrodynamics, the inorganic suspended solids (ISS) were chosen to show that the three-dimensional model with and without suspended solids gives different results as a result of differences in density structure. The density structure is affected by the water quality dynamics because suspended solids affect light penetration and hence temperature.

In the model, the inorganic suspended solids are lost in the system by settling with a user defined settling velocity $\left(\omega_{I S S}\right)$. Inorganic suspended solids were connected with hydrodynamic and temperature computations through the equation of state. Therefore, increase or decrease inorganic suspended solids leads to change the water density. Also, inorganic suspended solids affect temperature due to reducing the amount of light penetrated into water. The model links the inorganic suspended solids with the temperature sources/sinks by using a light extinction coefficient associated with the inorganic suspended solids in addition to that of water.

Thus, the source and sink term of inorganic suspended solids $\left(S_{I S S}\right)$ is

$$
S_{I S S}=-\omega_{I S S} \frac{\partial \emptyset_{I S S}}{\partial z}
$$


Where:

$z=$ the grid layer thickness, $\mathrm{m}$

$\omega_{I S S}=$ the settling velocity, $\mathrm{m} \mathrm{sec}^{-1}$

$\emptyset_{I S S}=$ the inorganic suspended solids concentration, $\mathrm{g} \mathrm{m}^{-3}$

In order to show this linkage, a $100 \mathrm{~g} \mathrm{~m}^{-3}$ inorganic suspended solids concentration was added to the major inflow (Clear Branch Creek) of the Laurance Lake reservoir continuously over the simulation period. Using a light extinction due to inorganic suspended solids of $0.1 \mathrm{~m}^{-1} /\left(\mathrm{g} \mathrm{m}^{-3}\right)$ and zero initial inorganic suspended solids concentration, the vertical temperature profiles with and without inorganic suspended solids in addition to vertical inorganic suspended solids profiles were plotted for selected times as shown in Figure 4-53. As the inorganic suspended solids changes over time, the vertical temperature distribution changes too. This impact on temperature depends on the waterbody conditions and becomes noticeable when the system undergoes stratified/nonstratified condition. 

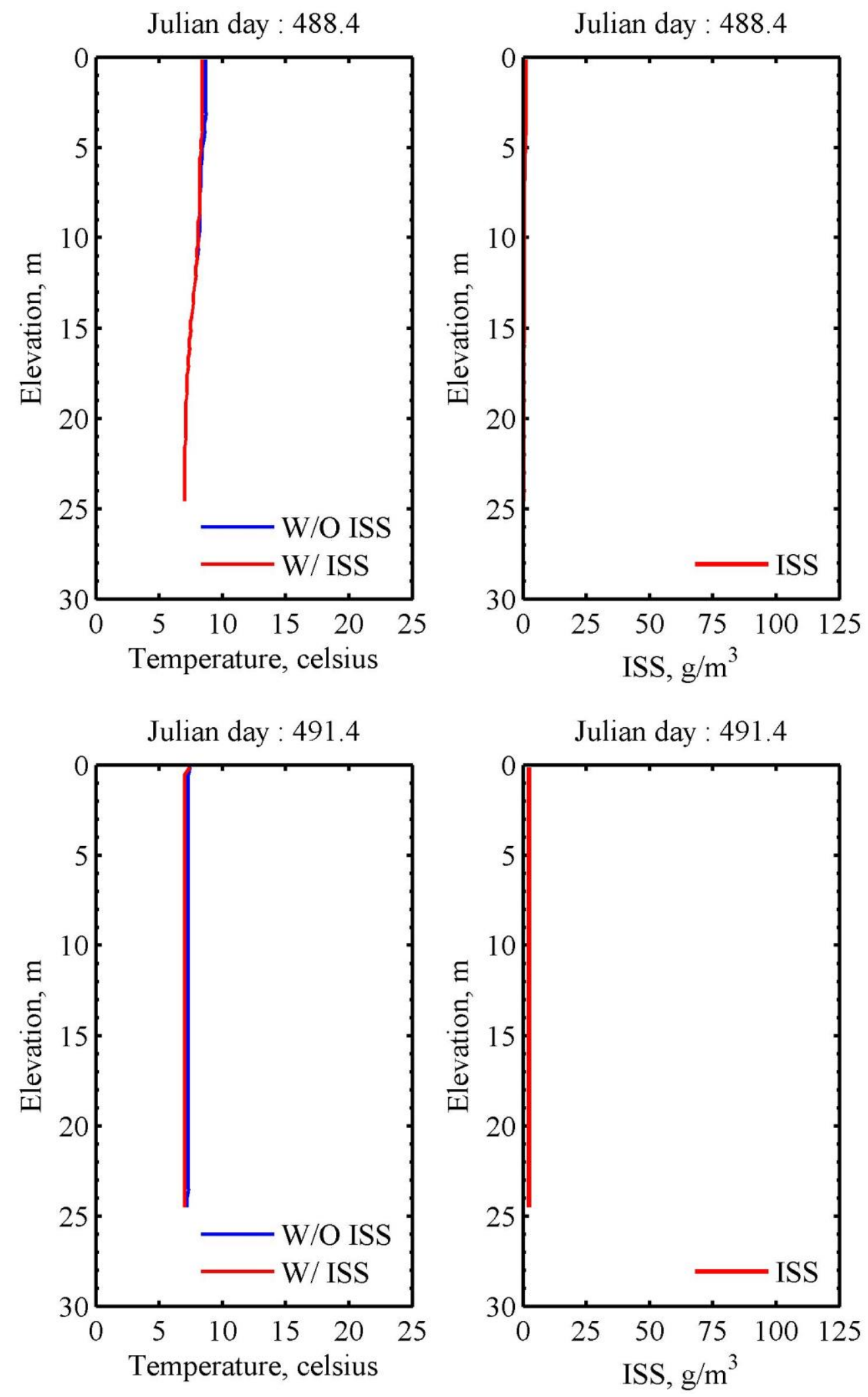

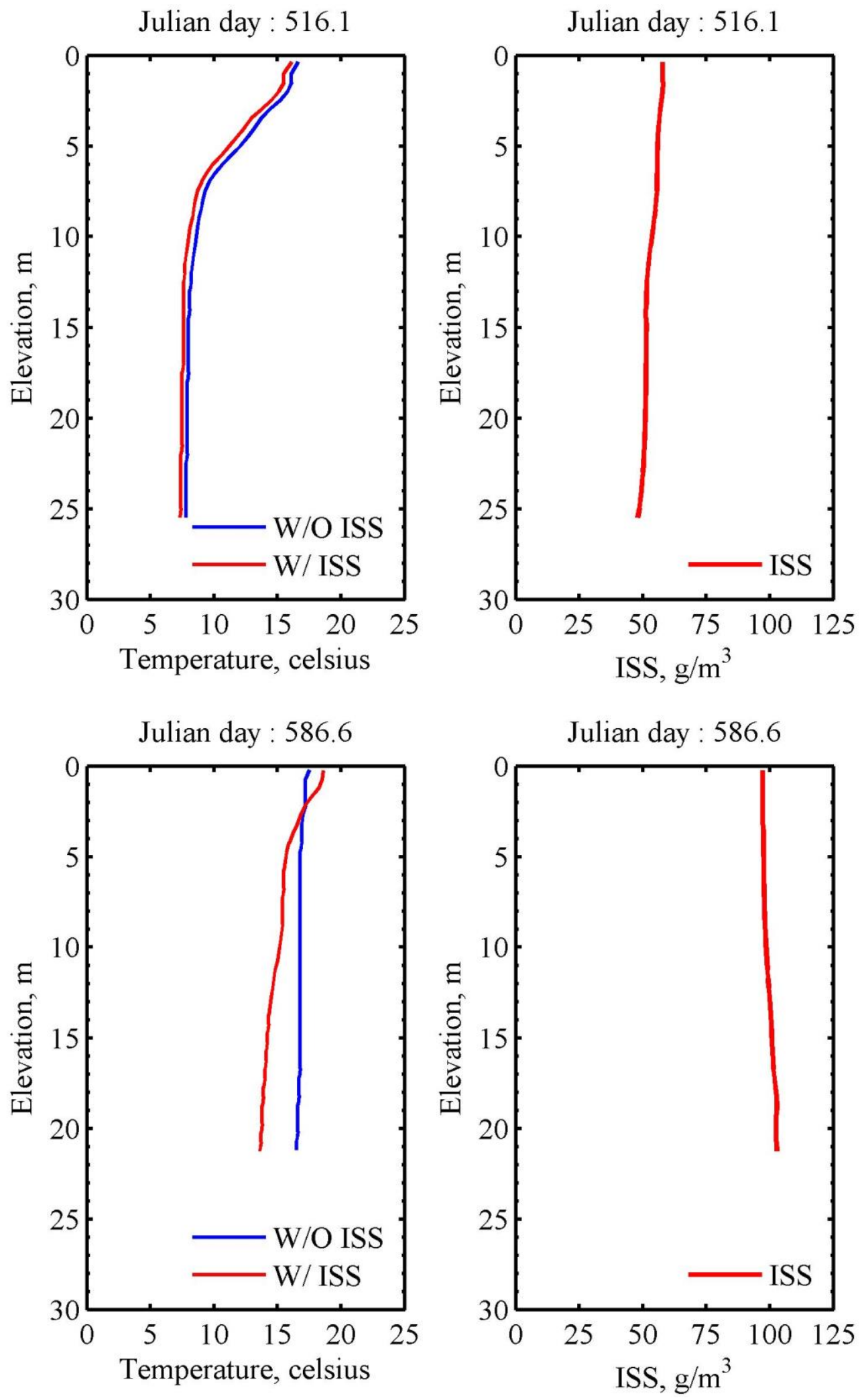

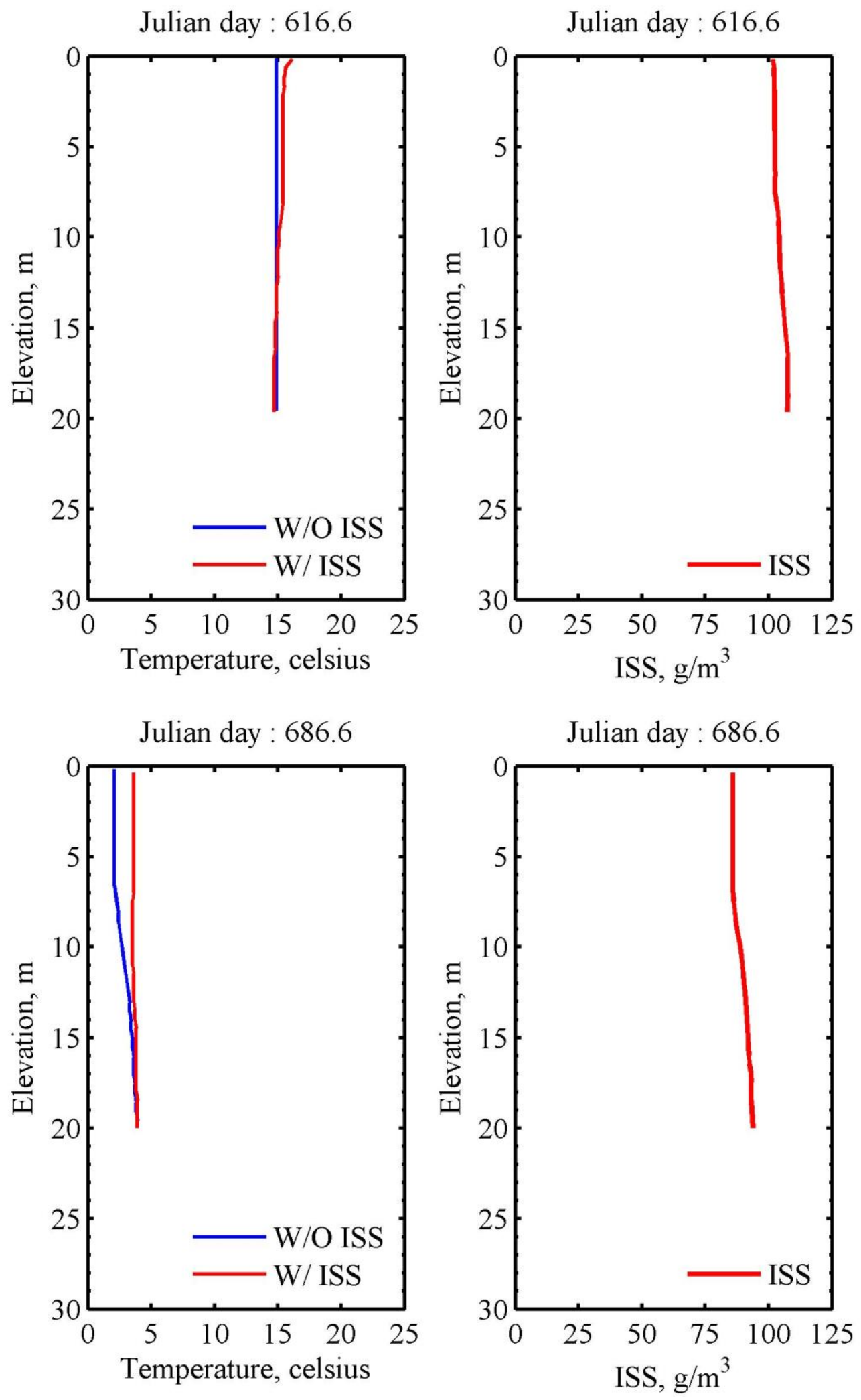

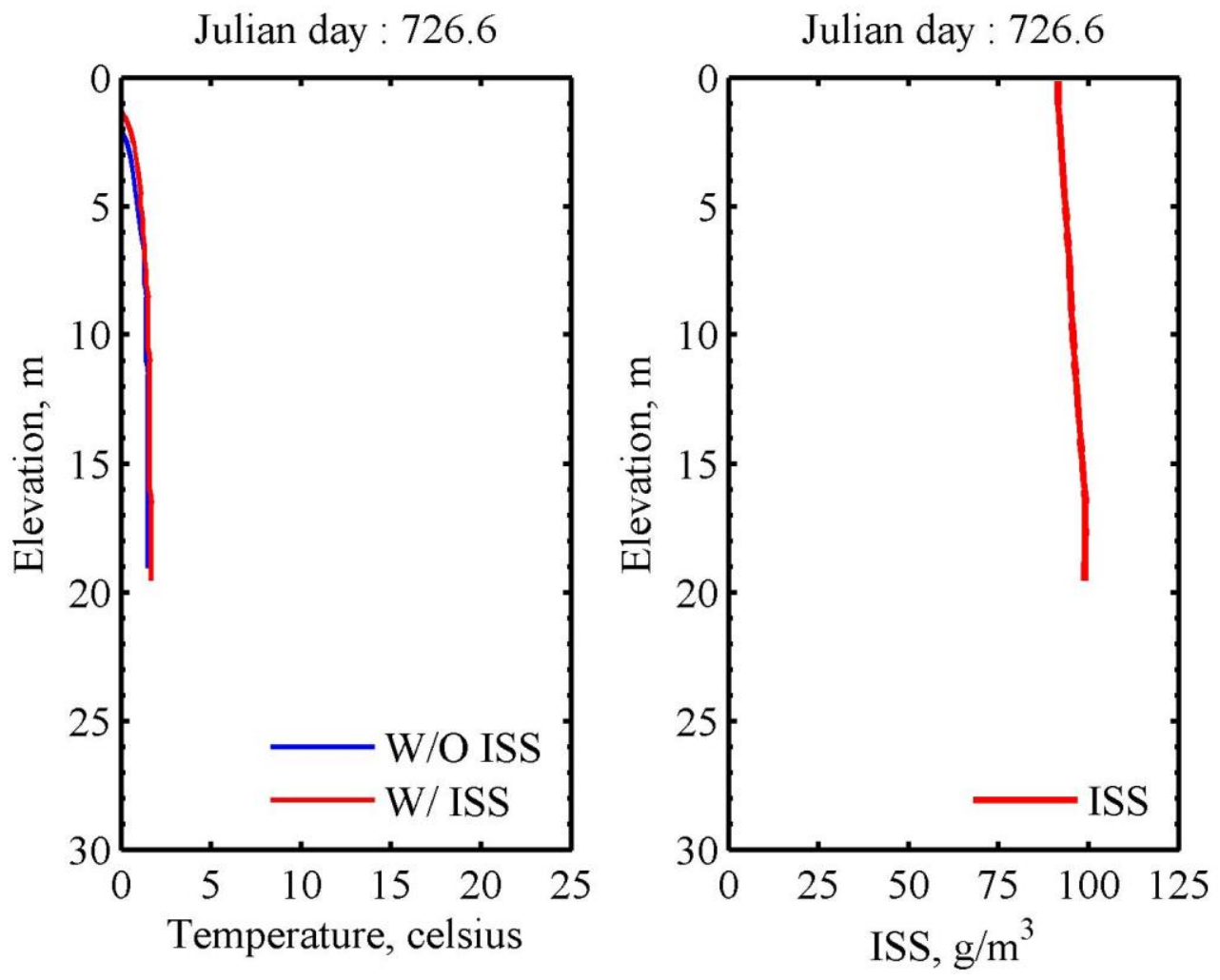

Figure 4-53. Laurance Lake model predictions of vertical ISS profiles in addition to the associated temperature distribution with and without ISS 


\subsection{Case Study 3: Cooper Creek Reservoir}

In order to show the model's ability to transfer heat between the onset of stratification to fall overturn as well as dissolved oxygen levels, a model was developed to simulate water level, temperature and dissolved oxygen in Cooper Creek Reservoir. The transition from stratified water column to well-mixed and returning back to the stratified conditions was simulated by comparing model predictions to vertical temperature profiles taken in the reservoir between 1998 and 1999. In this application, a spillway was added as a hydraulic structure to convey water from the reservoir at the dam location. Thus, the model predictions included the presence of two submerged withdrawals and an upper spillway flow. Additionally, the temperature dependent source/sink term of dissolved oxygen was computed, and then the model predictions of dissolved oxygen concentrations were compared with field data.

\subsubsection{Reservoir Background}

Cooper Creek Reservoir is a reservoir located in Douglas County, Oregon, US with an approximate elevation of $203.6 \mathrm{~m}$ and coordinates of Lat: 43.23 and Long: -123.37 . Figure 4-54 shows the location map of the reservoir and the surrounding terrain. Figure 4-55 shows the location of the reservoir within the drainage basin in addition to the dam location. The main inflow is from Cooper Creek at the south east end of the reservoir. The outflows are at the dam and by two outlet structures (Elevations: $192.02 \mathrm{~m}$ and $186.84 \mathrm{~m}$ ) and a spillway at an elevation of $203.73 \mathrm{~m}$ (see the sketch in Figure 4-56 for the dam outlets). The essential inflow/outflow data, reservoir bathymetry, water 
levels, and other input data required for modeling the reservoir were further described in Wells et al. (2000).

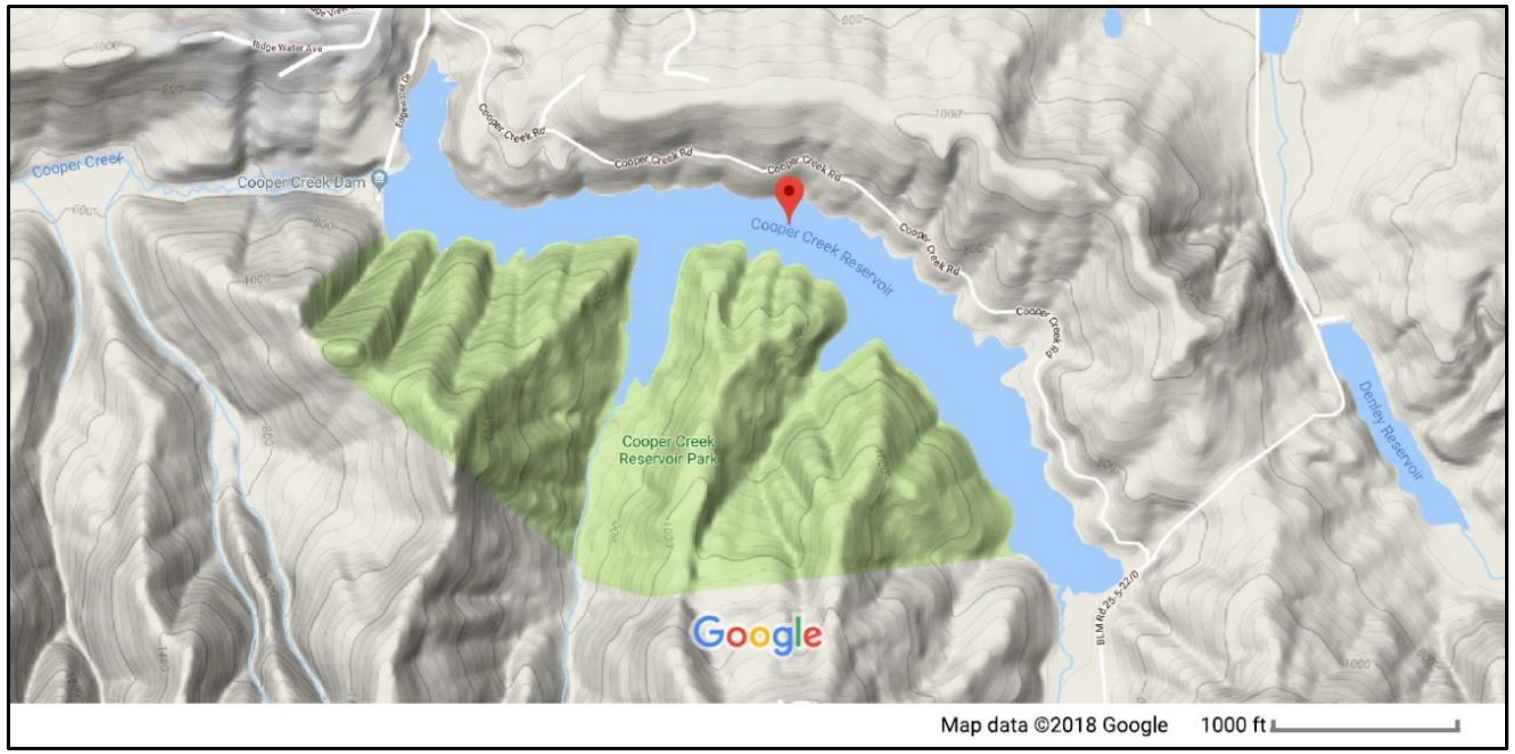

Figure 4-54. Cooper Creek Reservoir location (Google, 2018) 


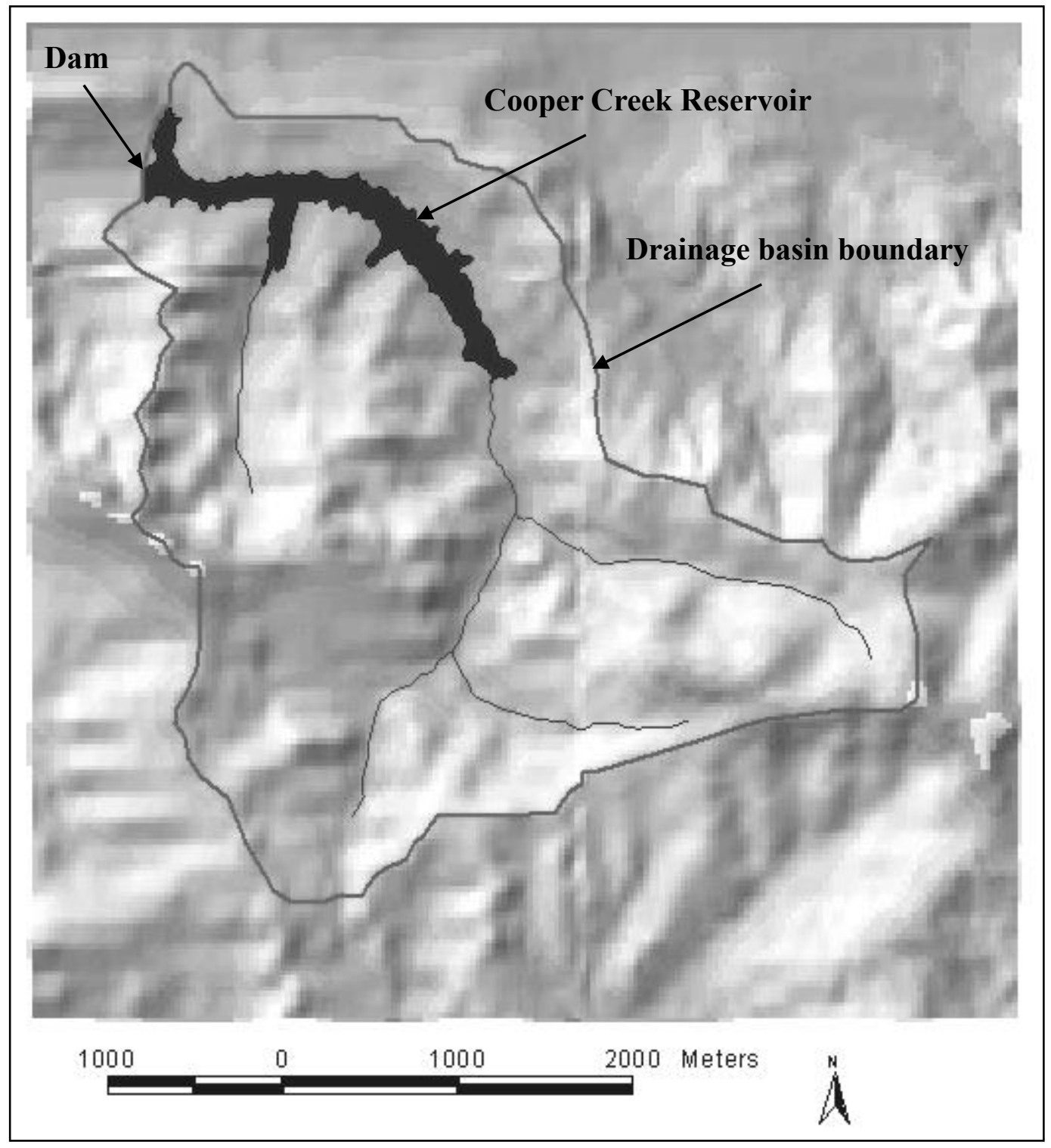

Figure 4-55. Cooper Creek Reservoir drainage basin 


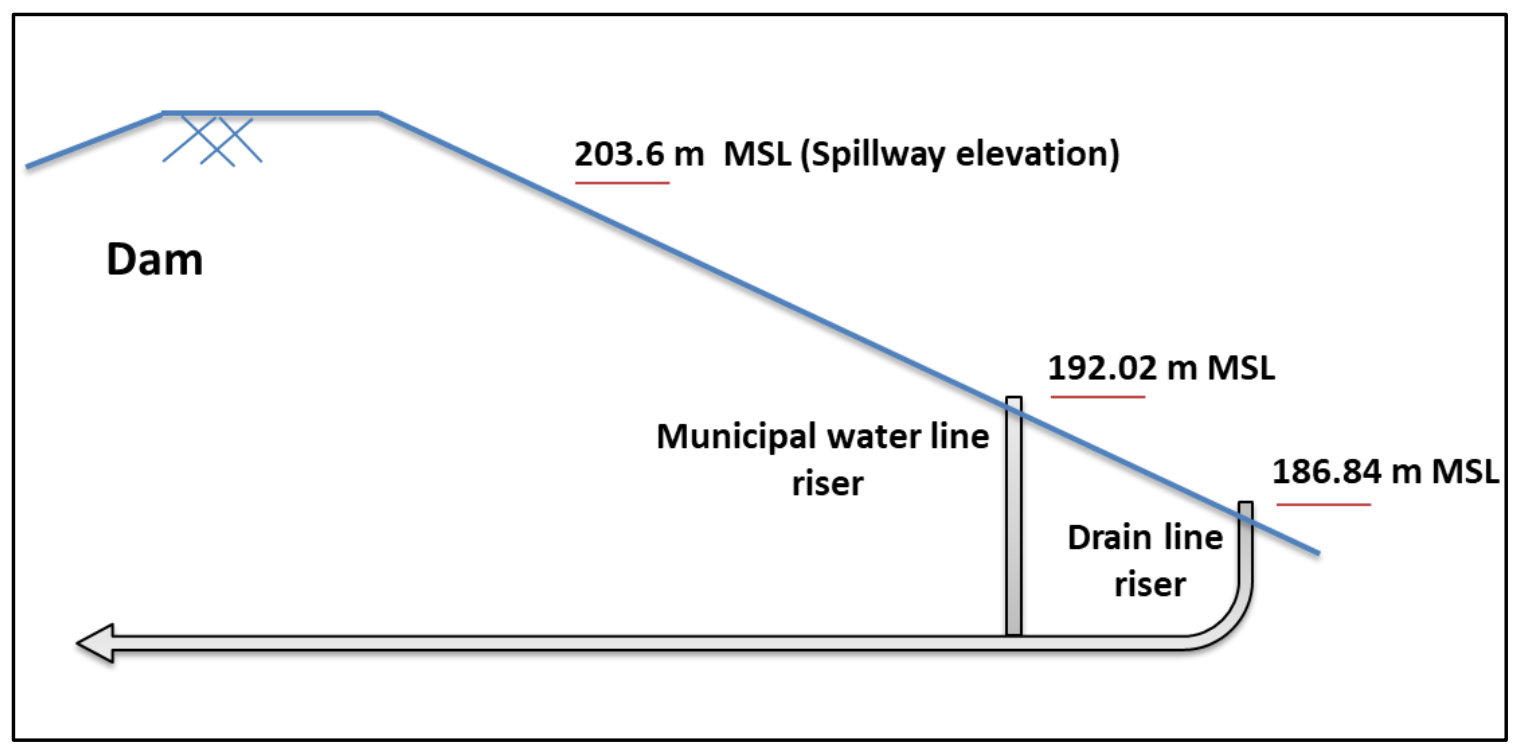

Figure 4-56. Cooper Creek Reservoir dam outlets

\subsubsection{Model Grid Development}

By using the model tools of development of the grid, the computational grid centers were overlaid above the contour plot as shown in Figure 4-57. Longitudinal increments of $\Delta x=$ $100 \mathrm{~m}$ and lateral increments of $\Delta y=50 \mathrm{~m}$ were used for model horizontal grid resolution, and a vertical increments $\Delta z=0.5 \mathrm{~m}$ were used for the model vertical grid resolution. An initial water surface elevation of $203.73 \mathrm{~m}$ was set at the layer of $k=k t=3$ based on the available data. 


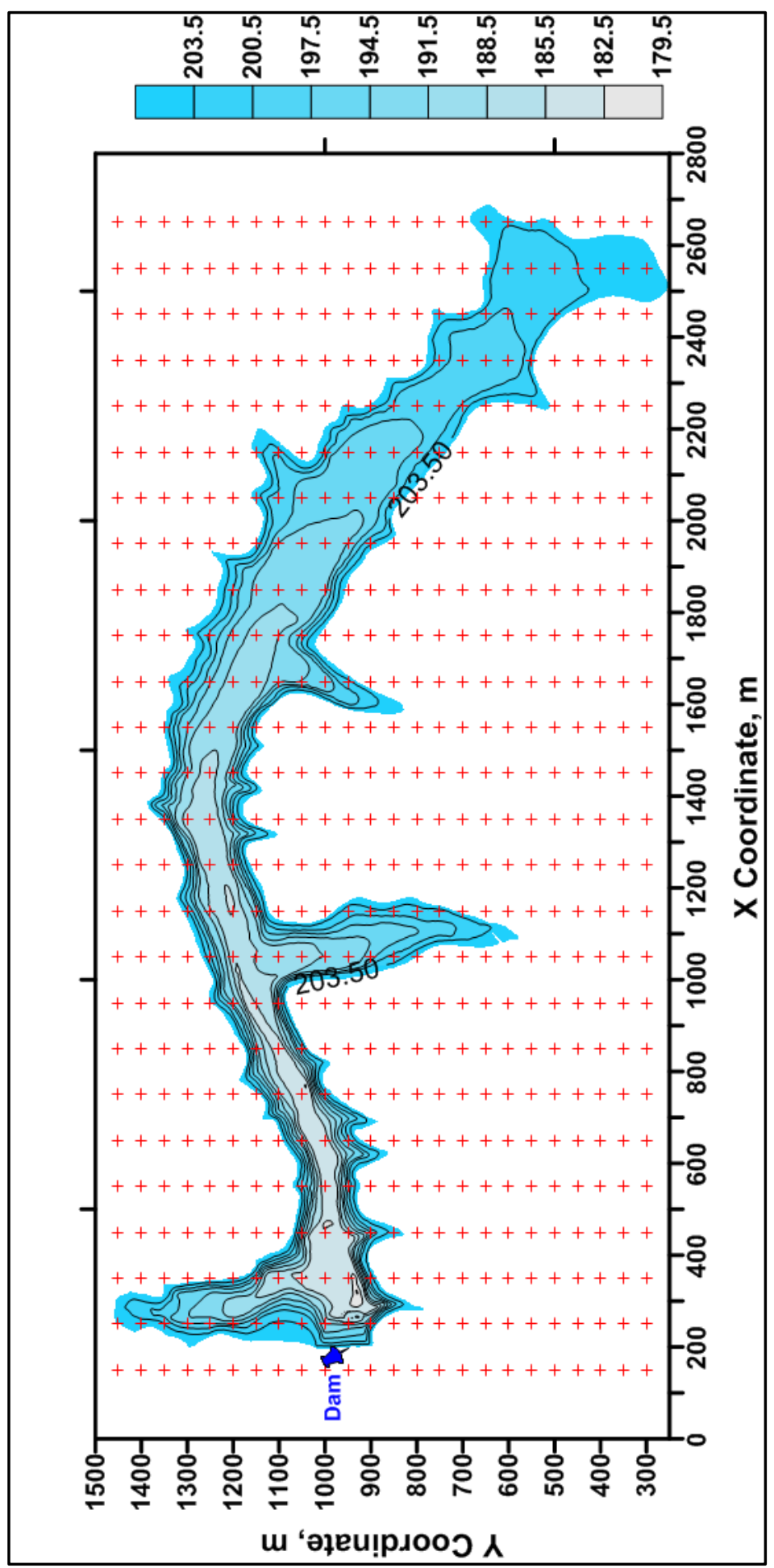

Figure 4-57. Cooper Creek Reservoir model computational grid 


\subsubsection{Flow Boundary Conditions and Meteorological Data}

Figure 4-58 and Figure 4-59 show the time series of the reservoir inflow from Cooper Creek and the corresponding temperature over the simulation period from May 7, 1998 to October 13, 1999 (Julian day: 127 - 651), respectively. In order to simulate the dissolved oxygen during this period of time, it was assumed that the inflow dissolved oxygen concentration of Cooper Creek was $8 \mathrm{mg} / \mathrm{L}$ close to the saturation state.

At the dam, there were two withdrawals in addition to a spillway, see Figure 4-56. Table 3 shows the outflows through the dam withdrawals based on the reservoir management requirements (an intermittent outflow for municipal water supply and a drain outflow for a week in fall to drop the water level).

The spillway flow was calculated in the model internally by using the following equation:

$$
Q_{\text {spillway }}=\alpha \Delta h^{\beta}
$$

Where:

$Q_{\text {spillway }}=$ the spillway flow, $\mathrm{m}^{3} / \mathrm{sec}$

$\Delta h=$ the water height above the weir crest, $\mathrm{m}$.

$\alpha$ and $\beta=$ fitted coefficients.

For Cooper Creek Reservoir case study, $\alpha$ and $\beta$ were $3.237 \mathrm{~m}^{3} / \mathrm{sec}$ and 0.373 , respectively, based on the designed flow rate curves, and the weir crest was set at an elevation of $203.73 \mathrm{~m}$ (the model initial water surface elevation). 
The essential meteorological data to run and calibrate the model (air temperature, dew point, wind magnitude and direction, and cloud cover) were available hourly from a NOAA station close to the reservoir. Figure 4-60 to Figure 4-64 show the meteorological data that were used in the model. In addition, the model calculated the required short solar radiation internally.

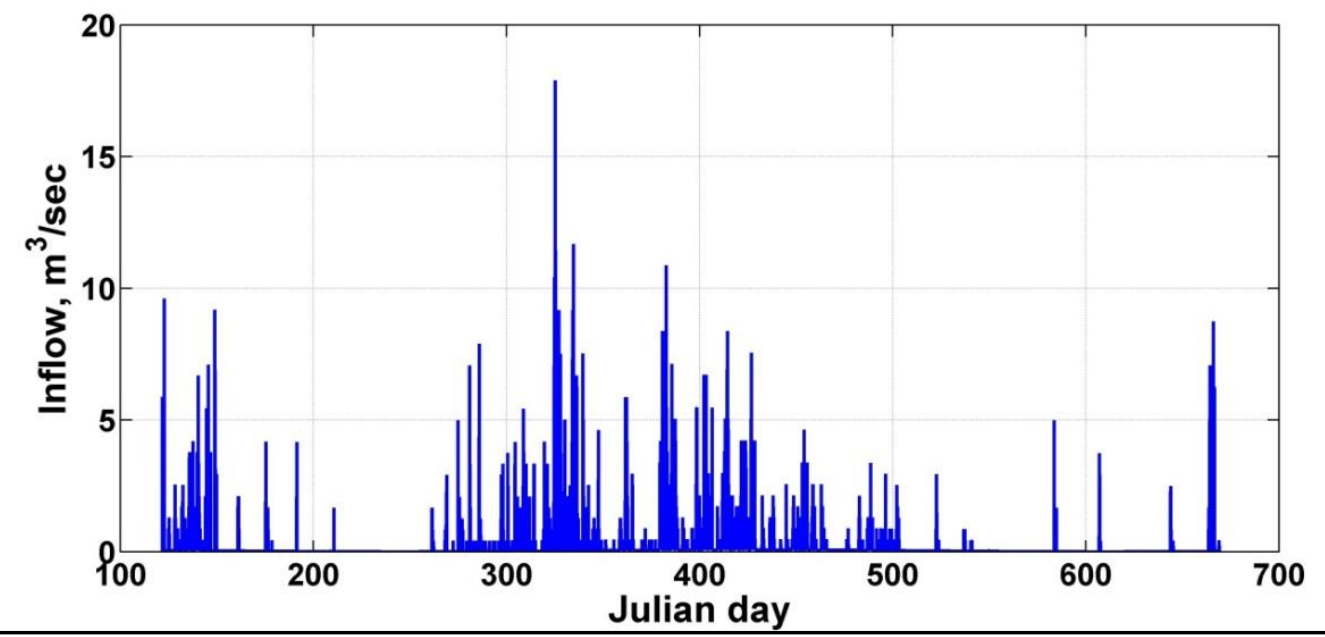

Figure 4-58. Creek Reservoir inflow from Cooper Creek

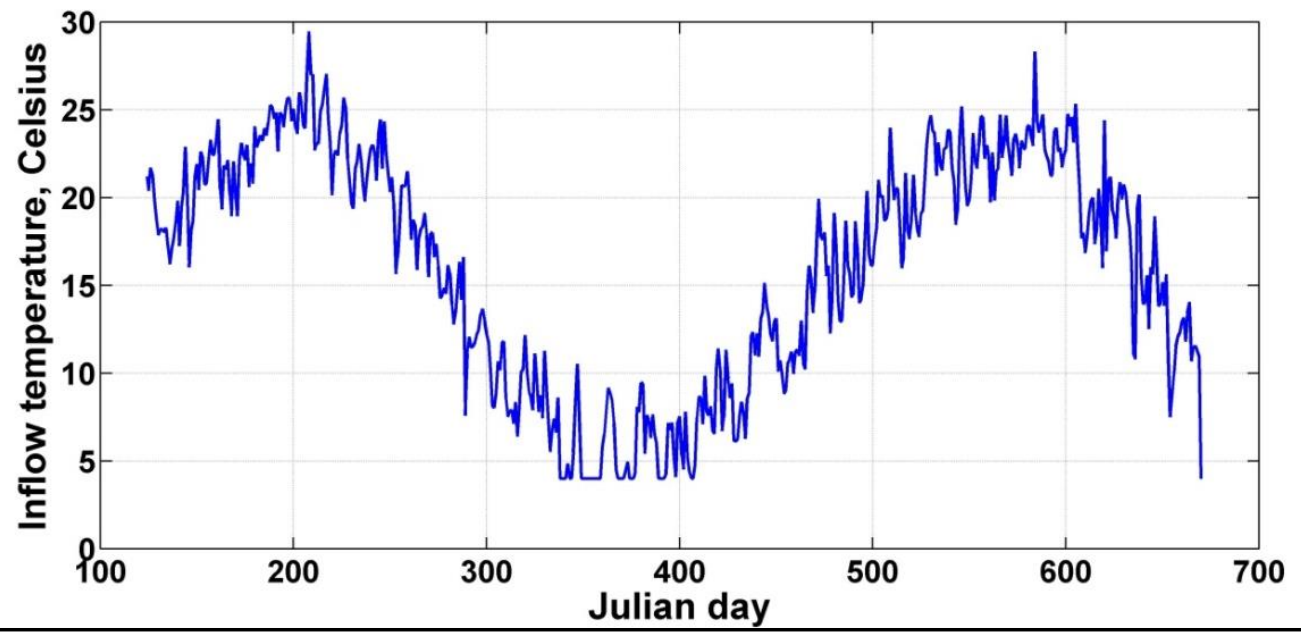

Figure 4-59. Cooper Creek Reservoir inflow temperature 
Table 3. Cooper Creek Reservoir dam withdrawals

\begin{tabular}{ccc}
\hline $\begin{array}{c}\text { Julian } \\
\text { day }\end{array}$ & $\begin{array}{c}\text { Municipal water } \\
\text { supply line } \\
\text { outflow }\left(\mathbf{m}^{\mathbf{3}} / \mathbf{s e c}\right)\end{array}$ & $\begin{array}{c}\text { Drain line outflow } \\
\left(\mathbf{m}^{\mathbf{3}} / \mathbf{s e c}\right)\end{array}$ \\
\hline 1 & 0 & 0 \\
196 & 0.0631 & 0 \\
247 & 0 & 0 \\
315 & 0 & 1.92 \\
322 & 0 & 0 \\
326 & 0.0631 & 0 \\
327 & 0 & 0 \\
359 & 0.0631 & 0 \\
360 & 0 & 0 \\
\hline 532 & 0.0631 & 0 \\
607 & 0 & 0 \\
624 & 0.0631 & 0 \\
625 & 0 & 0 \\
700 & 0 & 0 \\
\hline
\end{tabular}

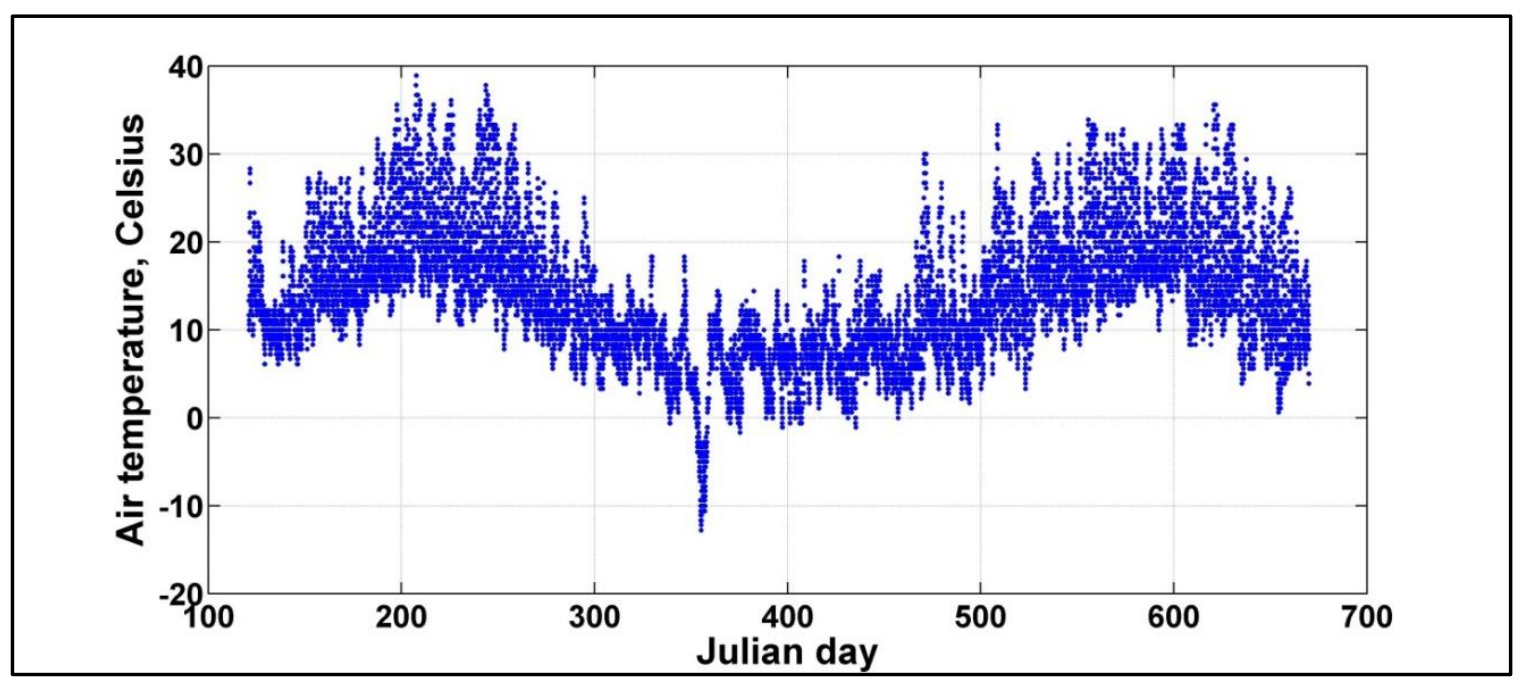

Figure 4-60. Air temperature input data of the Cooper Creek Reservoir model 


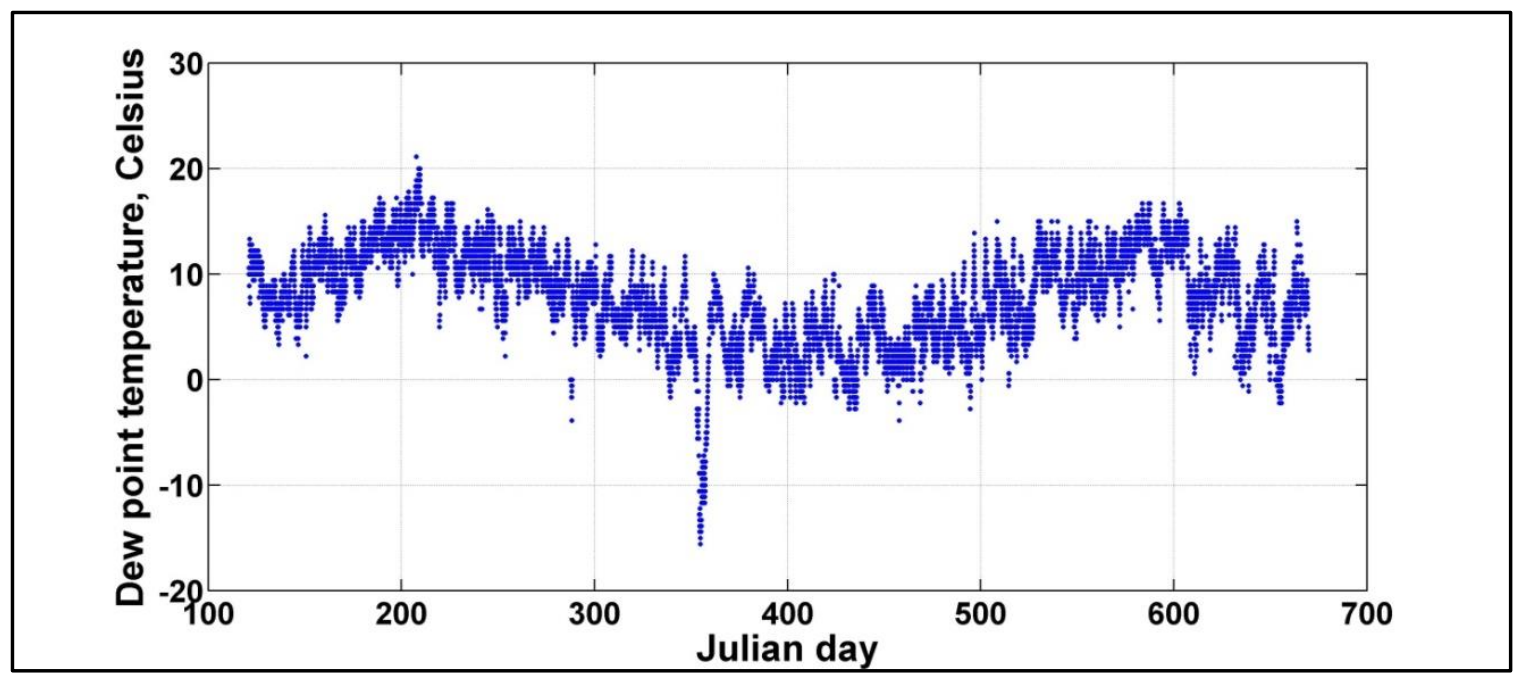

Figure 4-61. Dew point temperature input data of the Cooper Creek Reservoir model

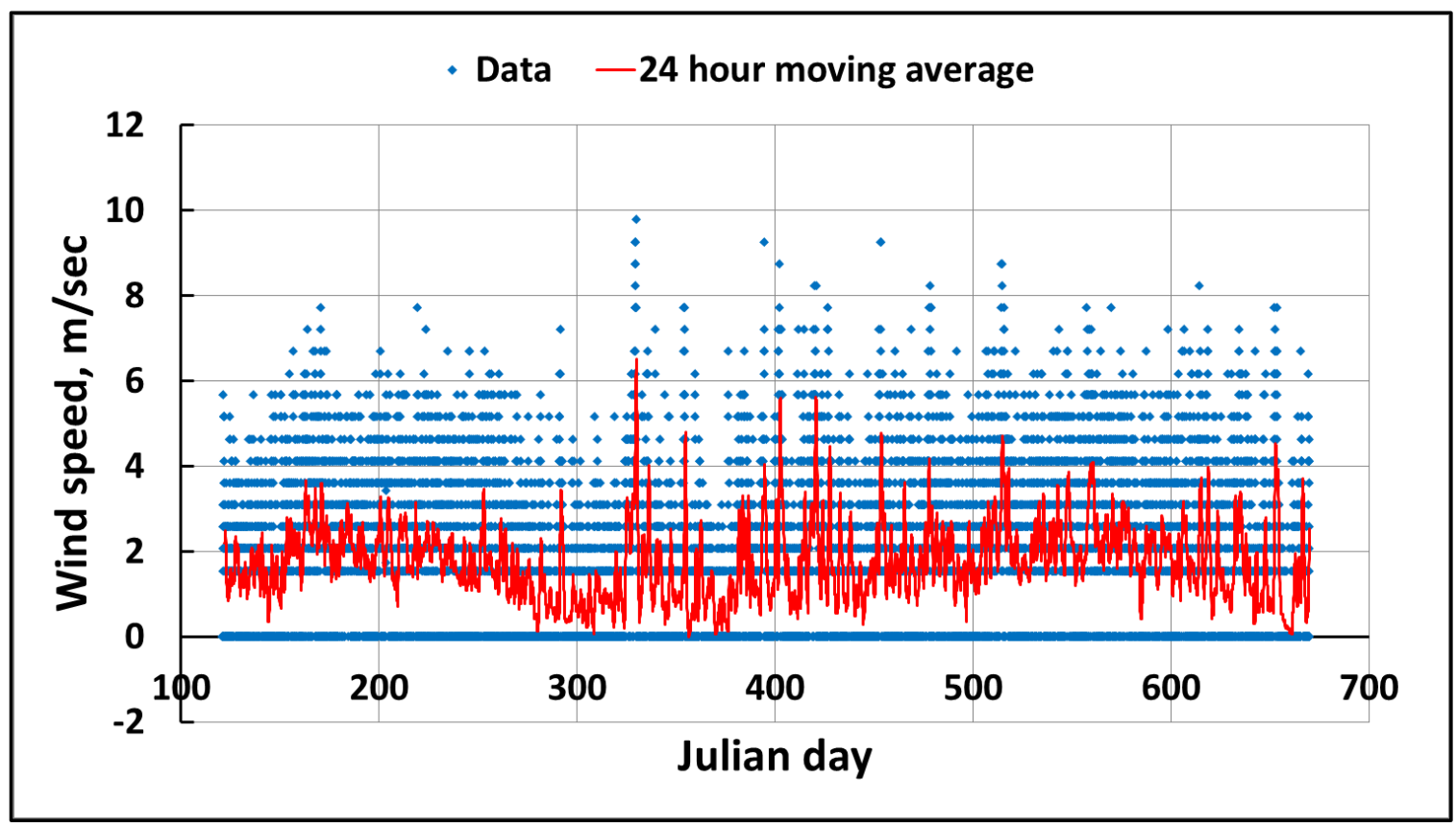

Figure 4-62. Wind speed input data of the Cooper Creek Reservoir model 


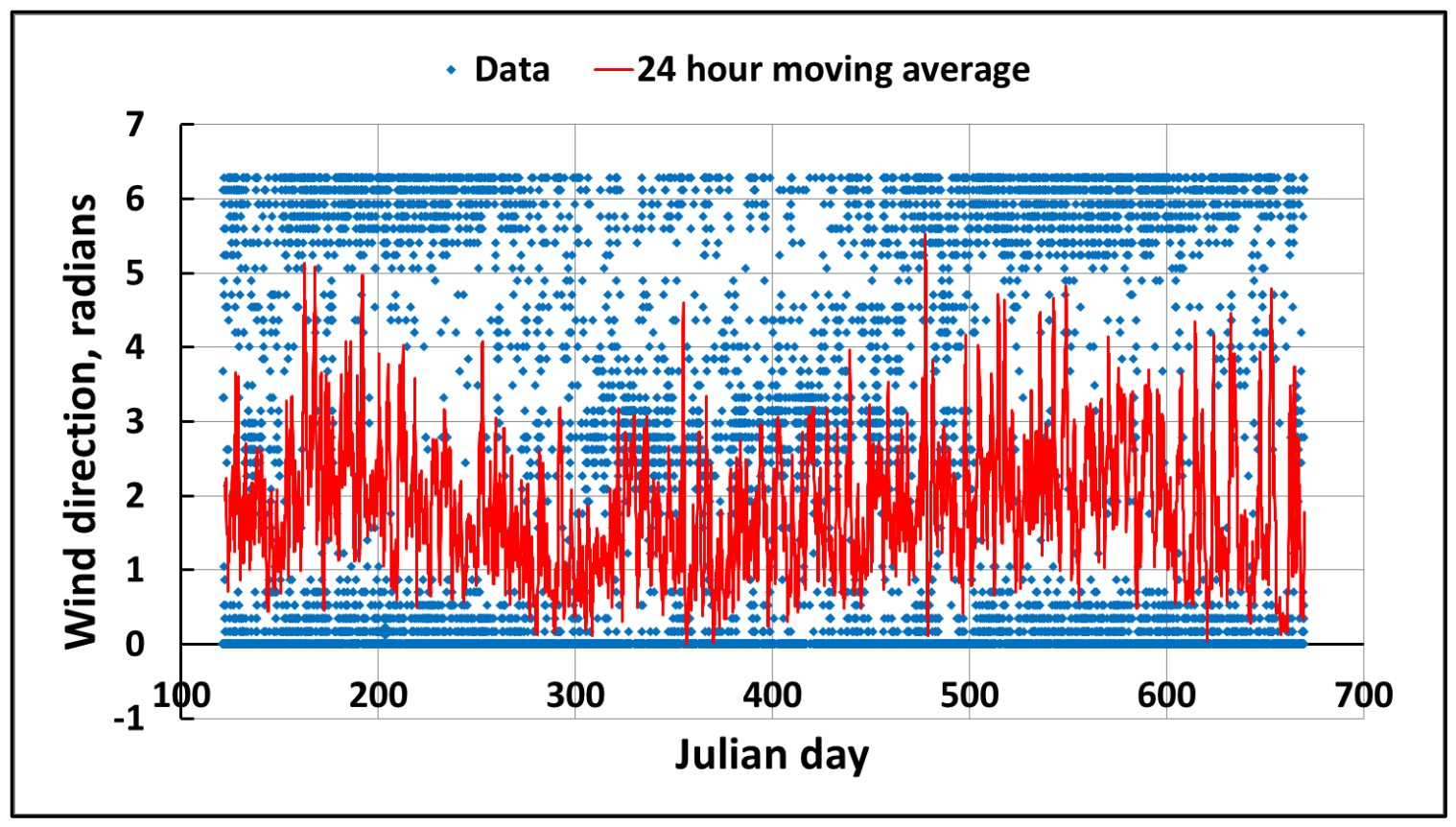

Figure 4-63. Wind direction input data of the Cooper Creek Reservoir model

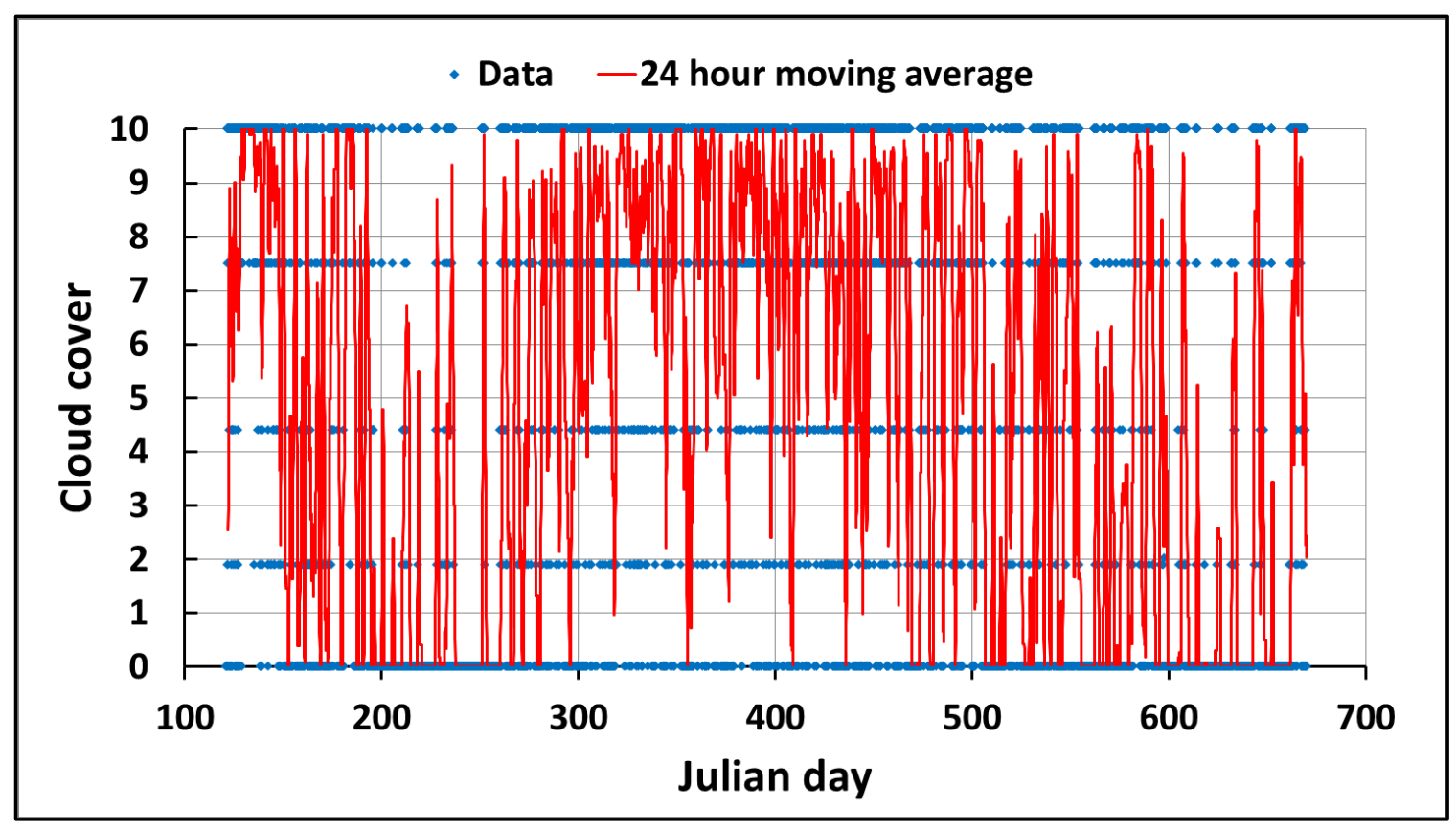

Figure 4-64. Cloud cover input data of the Cooper Creek Reservoir model 


\subsubsection{Dissolved Oxygen Source/Sink Computations}

Based on the computational interactions among hydrodynamics, temperature, and water quality constituents, the water quality computations do not affect the hydrodynamic and temperature computations since we are not simulating algae or other water quality state variables that could affect light transparency. Hydrodynamic computations and constituents' kinetics rate are affected by the temperature variations with time.

For the present modeling of dissolved oxygen in Cooper Creek Reservoir, reaeration coefficient $(K a)$ and sediment oxygen demand $(S O D)$ were used to calibrate the reservoir dissolved oxygen concentrations $(D O)$. Therefore, the source and sink term $\left(S_{D O}\right)$ that are required to be used in the numerical solution of the governing transport equation can be written as follows:

$$
S_{D O}=\left.k_{a}\left(D O_{s a t}-D O\right)\right|_{\text {reaeration term }}-\left.\frac{S O D}{H_{\text {layer }}}\right|_{\text {zero-order sediments term }}
$$

Where:

$S_{D O}=$ the dissolved oxygen source and sink term $\left(\mathrm{g} / \mathrm{m}^{3} / \mathrm{sec}\right)$,

$k_{a}=$ the reaeration coefficient $\left(\sec ^{-1}\right)$,

$D O_{\text {sat }}=$ the dissolved oxygen saturation concentration $\left(\mathrm{g} / \mathrm{m}^{3}\right)$,

$D O=$ the dissolved oxygen concentration $\left(\mathrm{g} / \mathrm{m}^{3}\right)$,

$S O D=$ the zero-order sediments oxygen demand $\left(\mathrm{gO}_{2} / \mathrm{m}^{2} / \mathrm{sec}\right)$, and 
$H_{\text {layer }}=$ the water layer thickness at which dissolved oxygen concentration is computed $(\mathrm{m})$.

Since there are many equations that could be used to determine the reaeration coefficient (Ka), the following equation (D. J. Smith, 1978) was an appropriate for the present Cooper Creek Reservoir model.

$$
K a=\frac{0.64+0.128 W^{2}}{H}
$$

Where $K a$ is the reaeration coefficient $\left(\mathrm{day}^{-1}\right), W$ is the wind speed $(\mathrm{m} / \mathrm{sec})$, and $H$ is the surface layer depth in (m).

Additionally, the saturation value of the dissolved oxygen was calculated as follows (Mortimer, 1981).

$$
D O_{\text {sat }}=P_{\text {alt }} e^{\{7.7117-1.31403(\ln [T+45.93])\}}
$$

Where:

$T$ is the water temperature in ${ }^{\circ} \mathrm{C}$, and $P_{a l t}$ is the elevation correction factor which was computed based on the waterbody elevation (Elv) in Kilometers above sea level (Mortimer, 1981).

$$
P_{\text {alt }}=\left[1-\frac{E l v}{44.3}\right]
$$




\subsubsection{Temperature Rate Multipliers}

All decay rates that govern biological processes over time vary with temperature. The effect of temperature variation on the decay rate was represented in the model by a temperature rate multiplier, $\lambda_{T}$. For each decay process, there is a temperature rate multiplier. This temperature rate multiplier varies non-linearly with temperature.

Thornton and Lessem (1978) developed an algorithm to express the rate multiplier as a function of temperature:

$$
\begin{array}{ll}
\lambda_{T}=0 & T \leq T_{1} \\
\lambda_{T}=\frac{K_{1} e^{\gamma_{1}\left(T-T_{1}\right)}}{1+K_{1}\left(e^{\gamma_{1}\left(T-T_{1}\right)}-1\right)} & T>T_{1} \\
\lambda_{T}=\frac{K_{4} e^{\gamma_{2}\left(T_{4}-T\right)}}{1+K_{4}\left(e^{\gamma_{2}\left(T_{4}-T\right)}-1\right)} & T<T_{4} \\
\lambda_{T}=0 & T \geq T_{4}
\end{array}
$$

Where:

$\gamma_{1}=\frac{1}{T_{2}-T_{1}} \ln \frac{K_{2}\left(1-K_{1}\right)}{K_{1}\left(1-K_{2}\right)}$

$\gamma_{2}=\frac{1}{T_{4}-T_{3}} \ln \frac{K_{3}\left(1-K_{4}\right)}{K_{4}\left(1-K_{3}\right)}$

$K_{1}, K_{2}, K_{3}$, and $K_{4}$ are the user-specified reaction rate multiplier corresponding to userspecified temperatures $T_{1}, T_{2}, T_{3}$, and $T_{4}$, respectively, in which $T_{2}$ and $T_{3}$ are the 
optimum temperatures range over which the biological process is at the maximum reaction rate.

Figure 4-65 shows an example to the curve generated by the algorithm of Thornton and Lessem (1978). The 2D CE-QUAL-W2 model proposes $K_{1}=0.1$ day $^{-1}$ at $T_{1}=5{ }^{\circ} \mathrm{C}$, $K_{2}=0.99$ day $^{-1}$ at $T_{2}=25{ }^{\circ} \mathrm{C}, K_{3}=0.99$ day $^{-1}$ at $T_{3}=35^{\circ} \mathrm{C}$, and $K_{4}=0.1$ day $^{-1}$ at $T_{4}=40{ }^{\circ} \mathrm{C}$. These proposed values are user defined parameters and can be adjusted in the model and are used for algae dynamics. But, for SOD decay and other processes that are non-algae processes like nitrification and BOD decay, only $K_{1}, K_{2}, T_{1}$, and $T_{2}$ were used as rate multipliers.

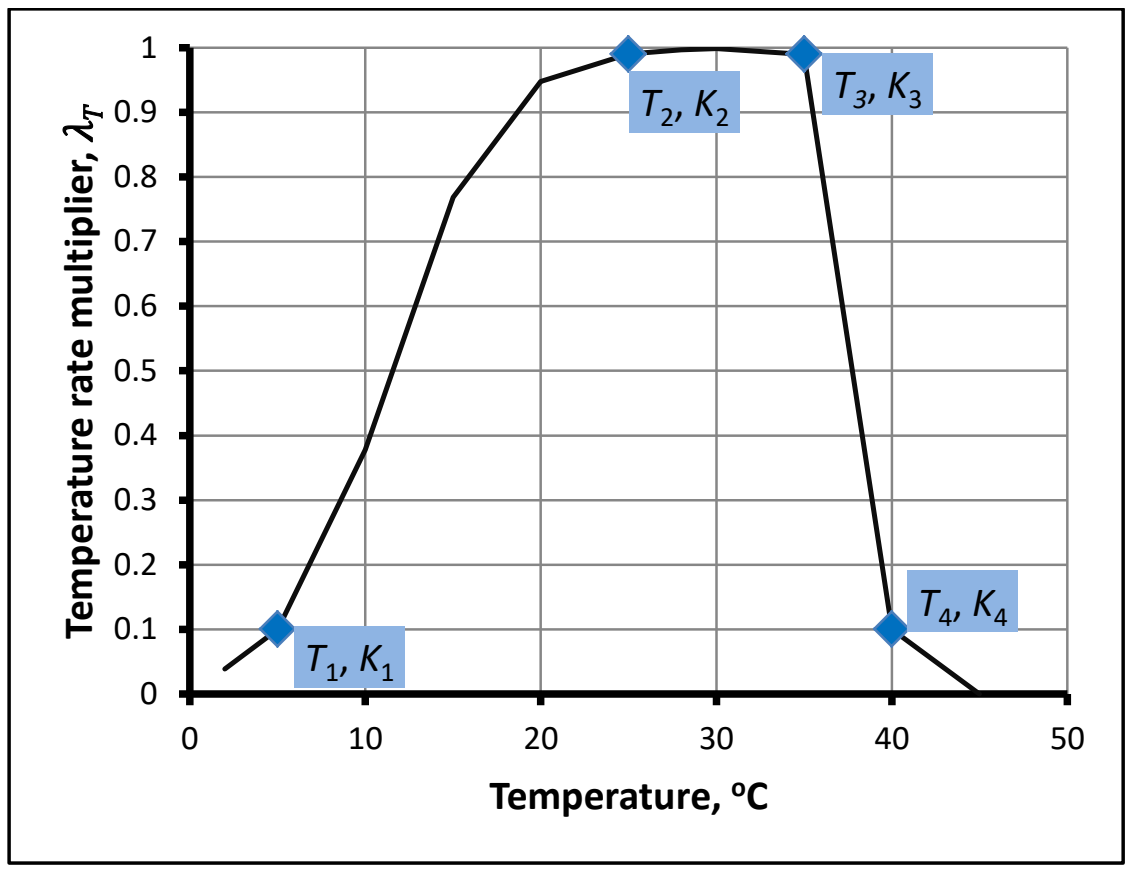

Figure 4-65. Temperature rate multipliers curve 


\subsubsection{Oxygen Limit Computation}

Dissolved oxygen is very important in aquatic ecosystems due to its direct effects on aerobic and anaerobic processes such as nitrification-denitrification process, organic matter decay, nutrients release by sediments, algal growth, and productivity. Therefore, the model controls processes that rely on dissolved oxygen by stopping decay processes as dissolved oxygen approaches zero and provides a smooth transition between aerobic and anaerobic processes as dissolved oxygen concentrations change.

Monod growth formulation was adopted for the gradual transition between oxic and anoxic conditions by introducing a reduction factor that varied between 0 and 1 (Cole \& Wells, 2017). Therefore, a reduction factor was calculated and multiplied by the considered temperature rate multiplier.

$$
\text { Reduction factor }=\frac{\phi_{D O}}{K_{D O}+\phi_{D O}}
$$

Where $K_{D O}$ is the dissolved oxygen half-saturation constant $\left(\mathrm{g} / \mathrm{m}^{3}\right)$.

$K_{D O}$ represents the dissolved oxygen limit when oxygen conditions are at $50 \%$ of their maximum. The higher the dissolved oxygen half-saturation constant is, the more gradual transition between oxic and anoxic conditions is. The model considers $K_{D O}$ as a userspecified constant. A value of $0.7 \mathrm{~g} / \mathrm{m}^{3}$ was used for $K_{D O}$ (Thomann \& Mueller, 1987) and set as the default in the model. 


\subsubsection{Cooper Creek Reservoir Model Calibration}

The simulation time between May 7, 1998 and October 13, 1999 includes two stratification periods in the summer 1998 and 1999, as well as the fall overturn of 1998. The model was run using a time step of $2 \mathrm{sec}$ and was first calibrated using the water level data. Figure 4-66 shows the model predictions of water levels compared to field data. The root mean square error and absolute mean error of the water level prediction was $0.175 \mathrm{~m}$ and $0.129 \mathrm{~m}$, respectively.

Figure 4-67 shows the spillway outflows. These outflows were determined internally by the model based on the water surface levels and the spillway crest level. When the water level goes above the spillway crest, the spillway was turned on. 


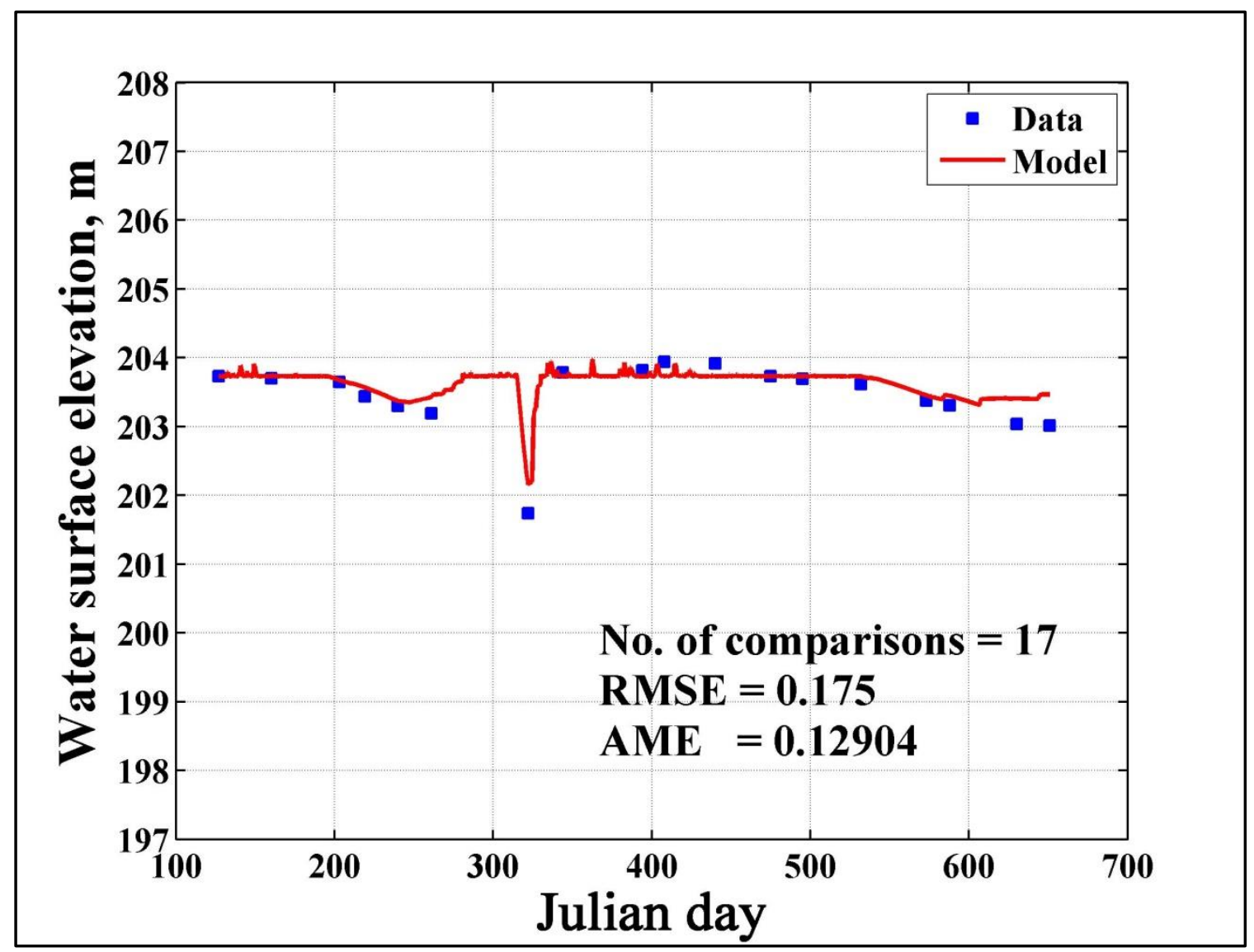

Figure 4-66. Cooper Creek Reservoir model predictions of water surface elevation compared with data using QUICKEST scheme 


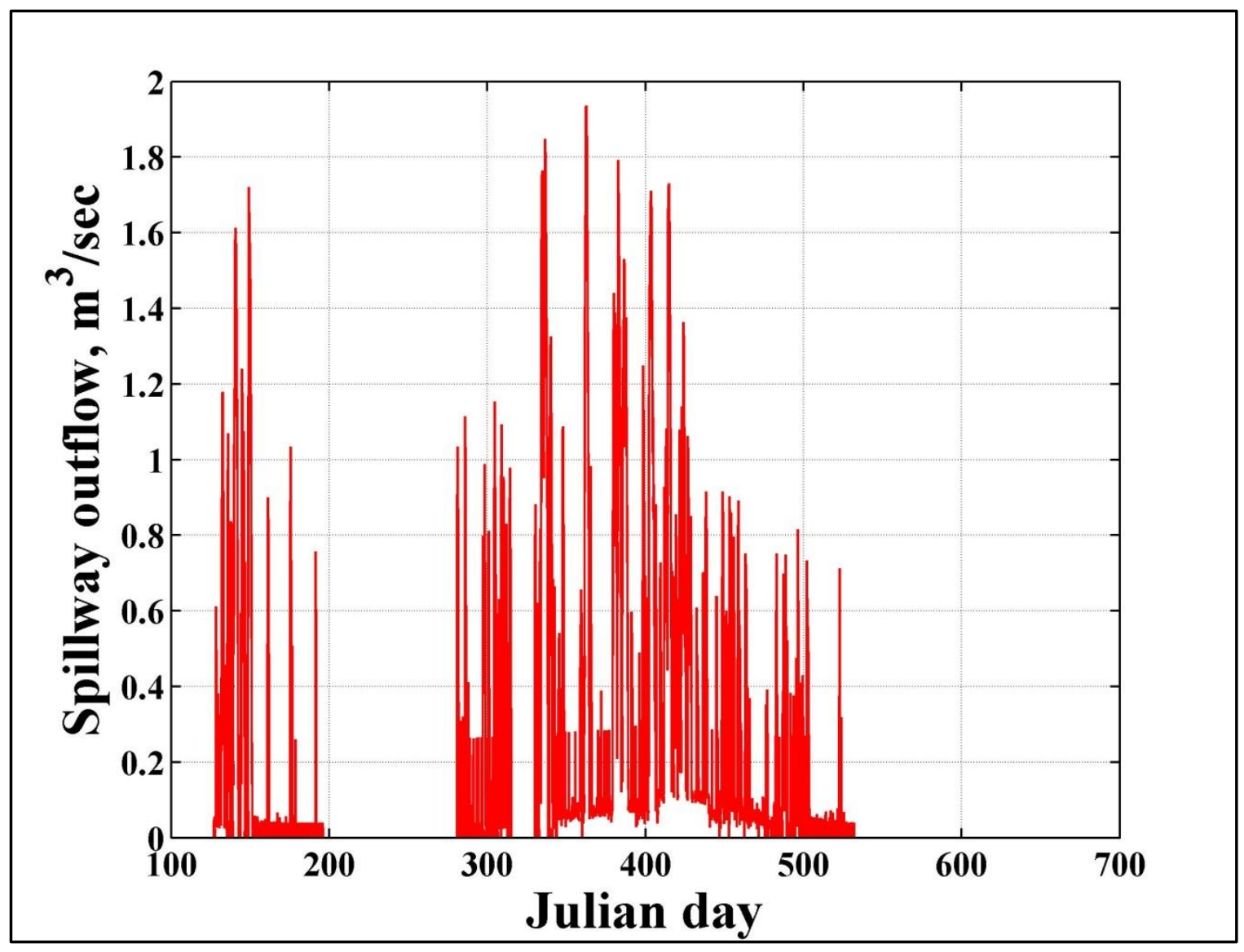

Figure 4-67. Cooper Creek Reservoir spillway outflow at the dam 
The model calibration involved comparing vertical profiles of temperature and dissolved oxygen to profile field data. The available data have five vertical profiles for temperature, three profiles in 1998 and two profiles in 1999. Figure 4-68 shows the model vertical temperature profile predictions compared to the field data. The overall absolute mean error (AME) of the temperature profiles was approximately $1.00{ }^{\circ} \mathrm{C}$.

Simultaneously, the dissolved oxygen concentrations were computed for the reservoir by applying the $K a$ equation and a maximum SOD of $1.3 \mathrm{gO}_{2} / \mathrm{m}^{2} /$ day. This value of SOD was varied with temperature with the maximum decay rate occurring at $30^{\circ} \mathrm{C}$ and $10 \%$ of the maximum decay rate occurring at $4{ }^{\circ} \mathrm{C}$. Starting the model run with an initial dissolved oxygen concentration of $4 \mathrm{~g} / \mathrm{m}^{3}$, the model predictions of dissolved oxygen concentration are shown in Figure 4-69. The error statistics for model predicted dissolved oxygen concentrations compared with field data were $1.32 \mathrm{~g} / \mathrm{m}^{3}$ overall AME.

Furthermore, the error statistics of the 2D CE-QWAL-W2 model for water level, temperature, and dissolved oxygen are shown in Table 4. Figure 4-70 shows the 2D model prediction of water level compared to data, and Figure 4-71 shows examples of the 2D model predictions for temperature and dissolved oxygen. 

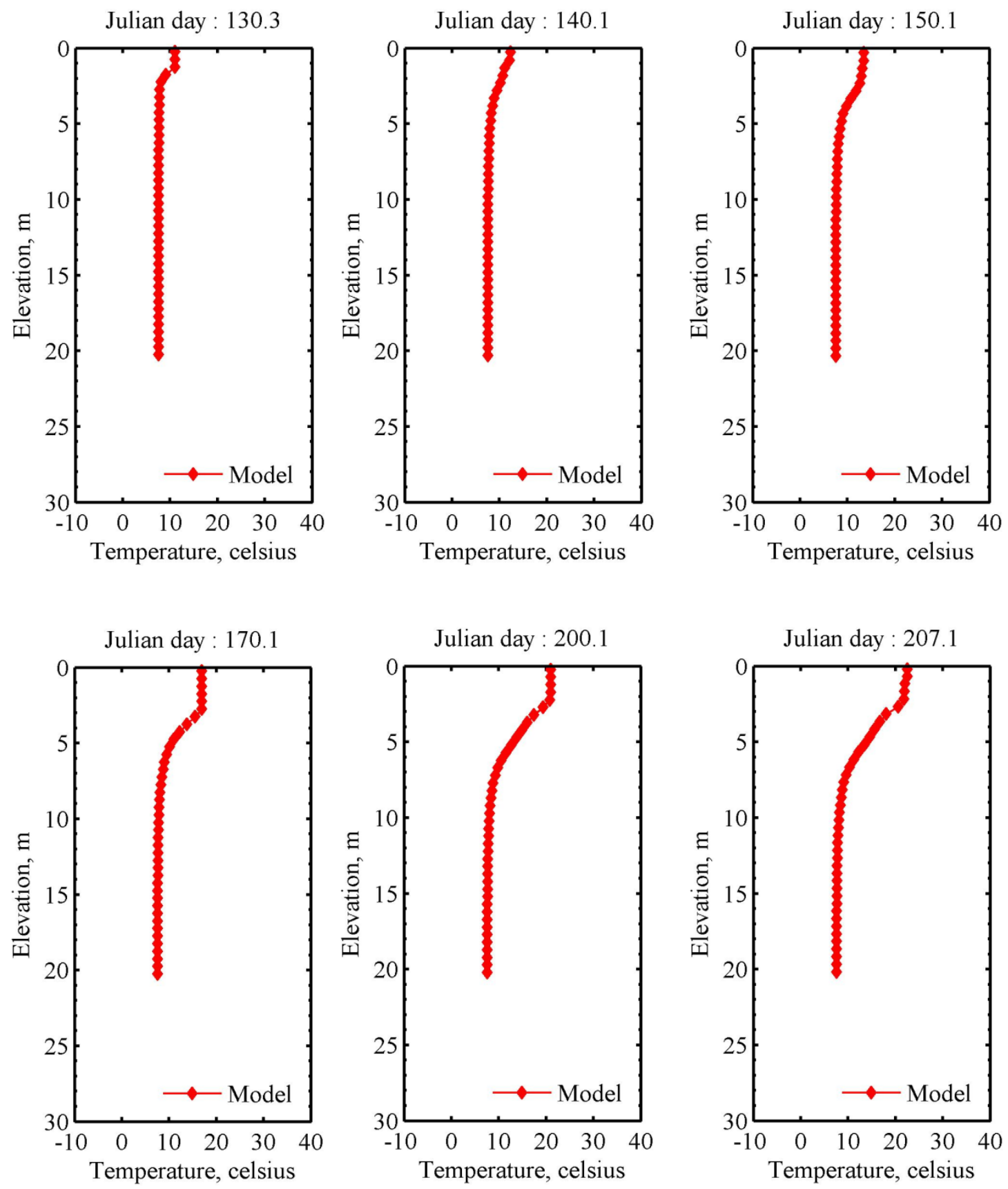

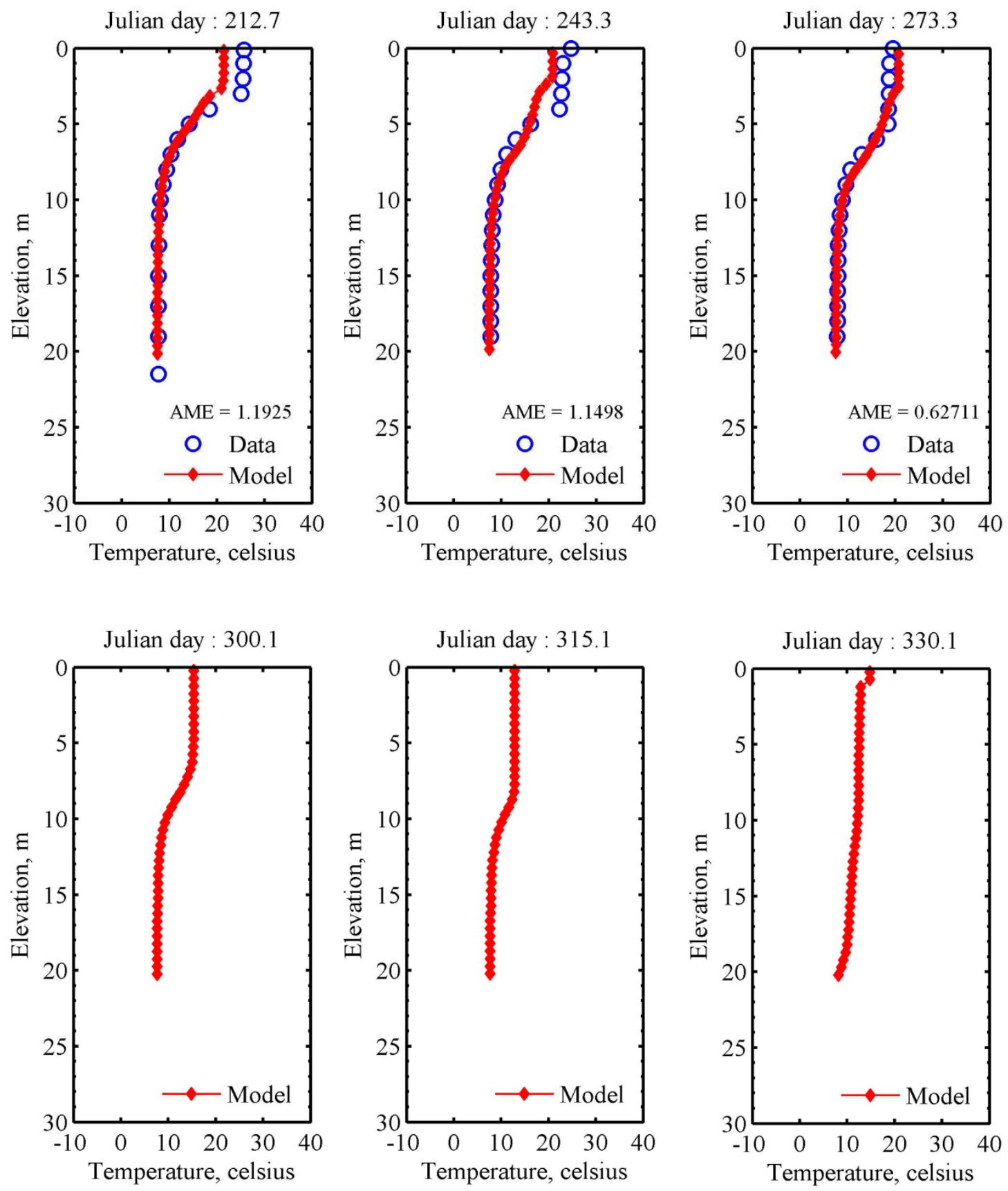

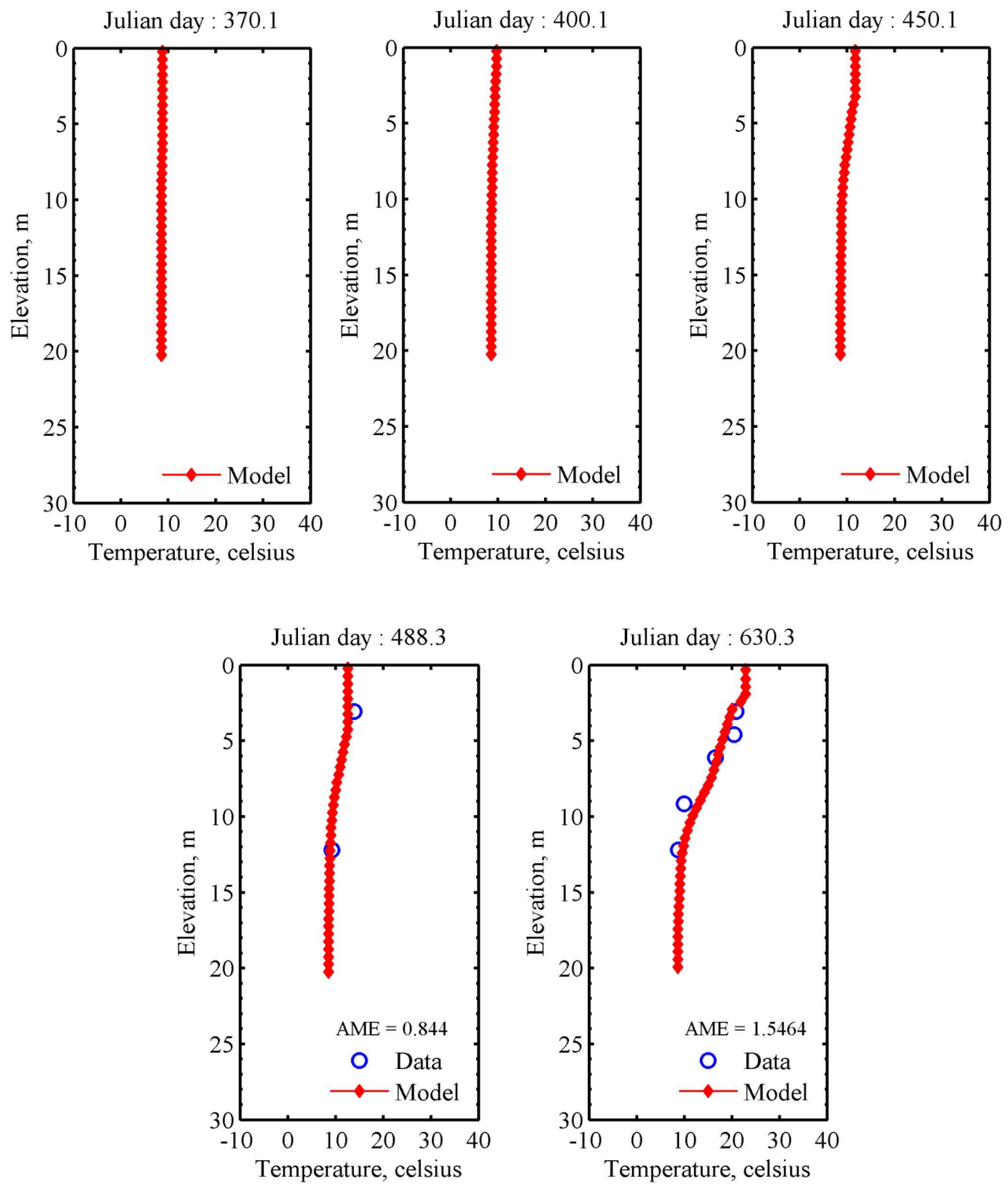

Figure 4-68. Cooper Creek Reservoir model predictions of vertical temperature profiles compared with field data 

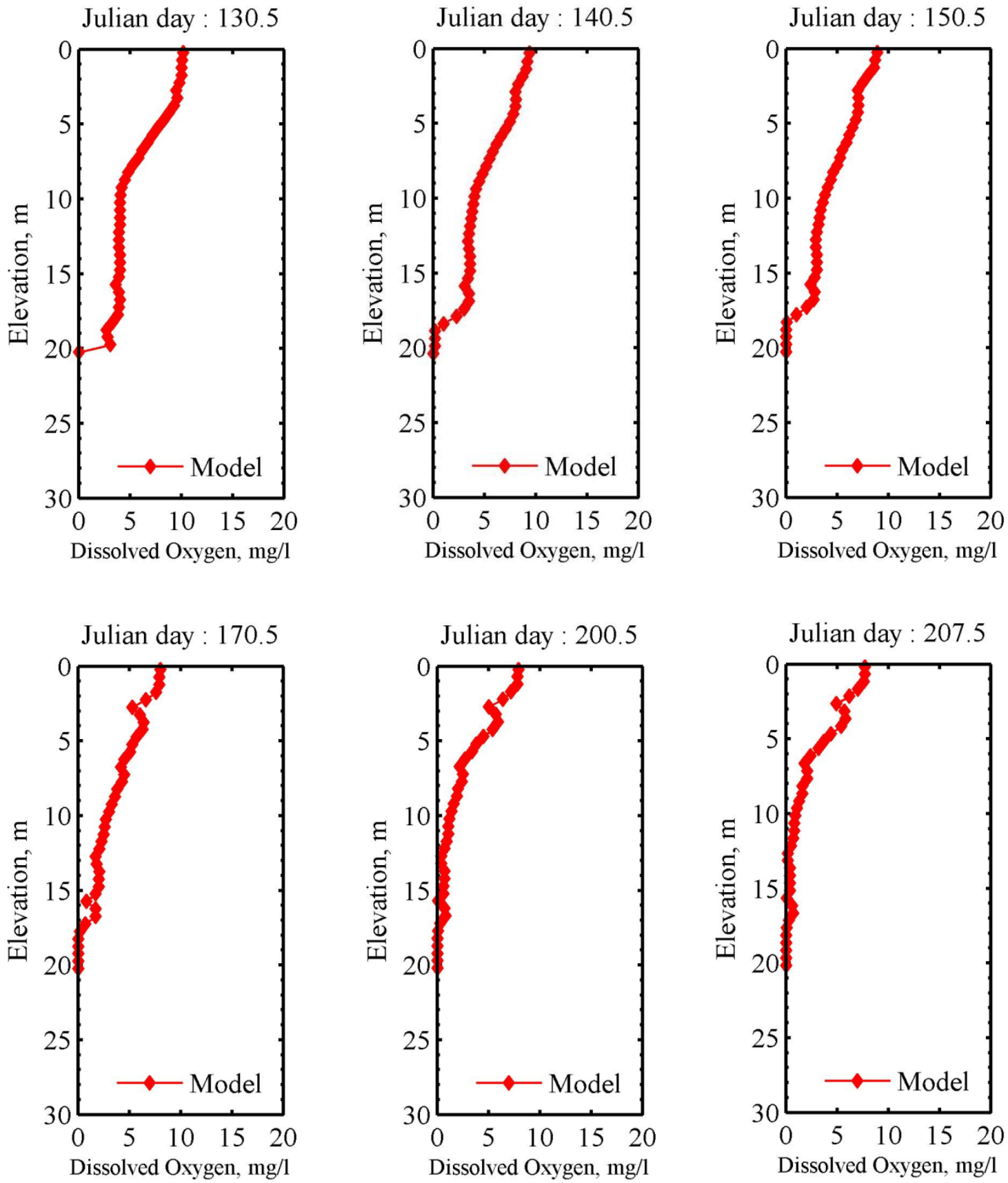

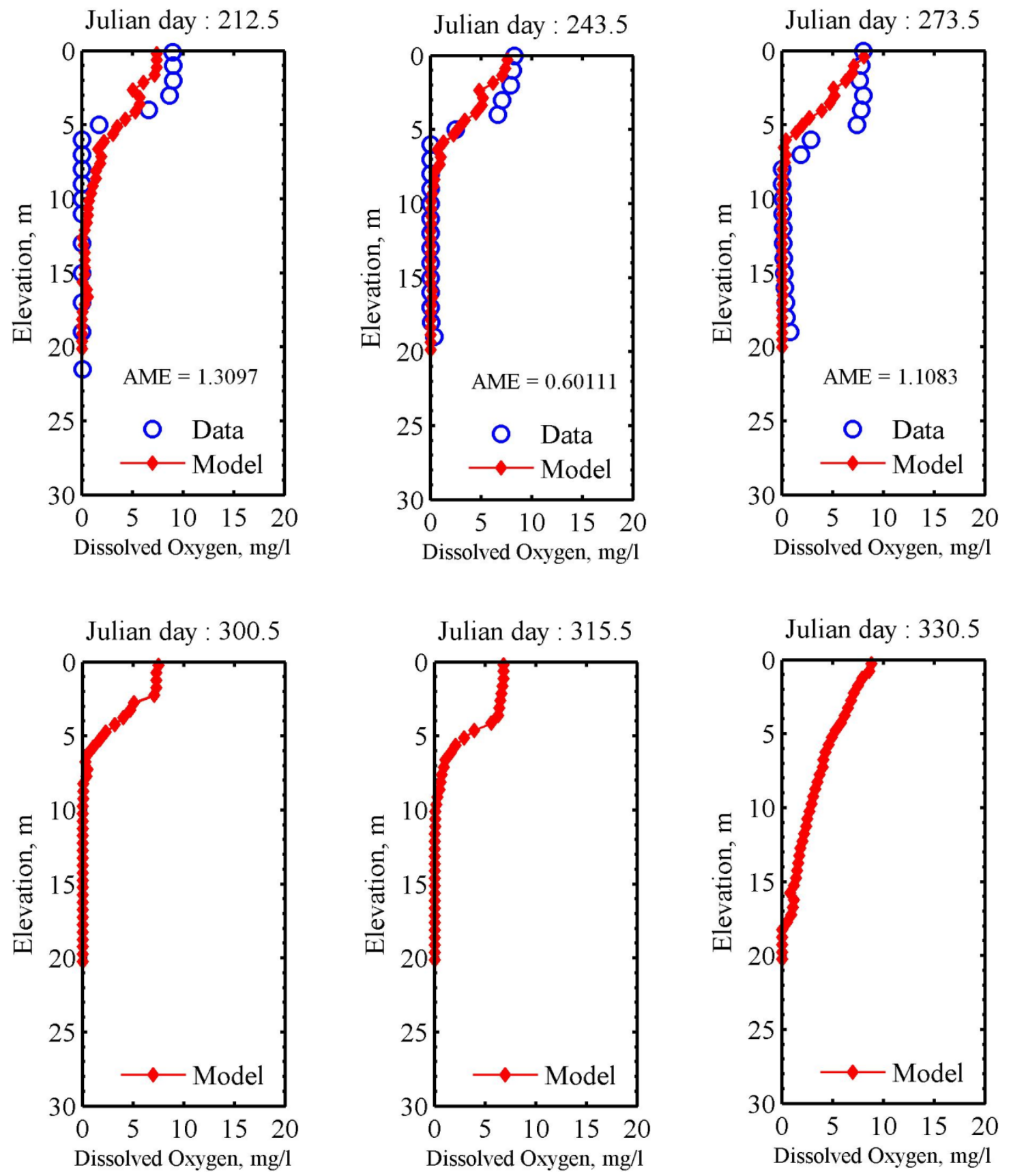

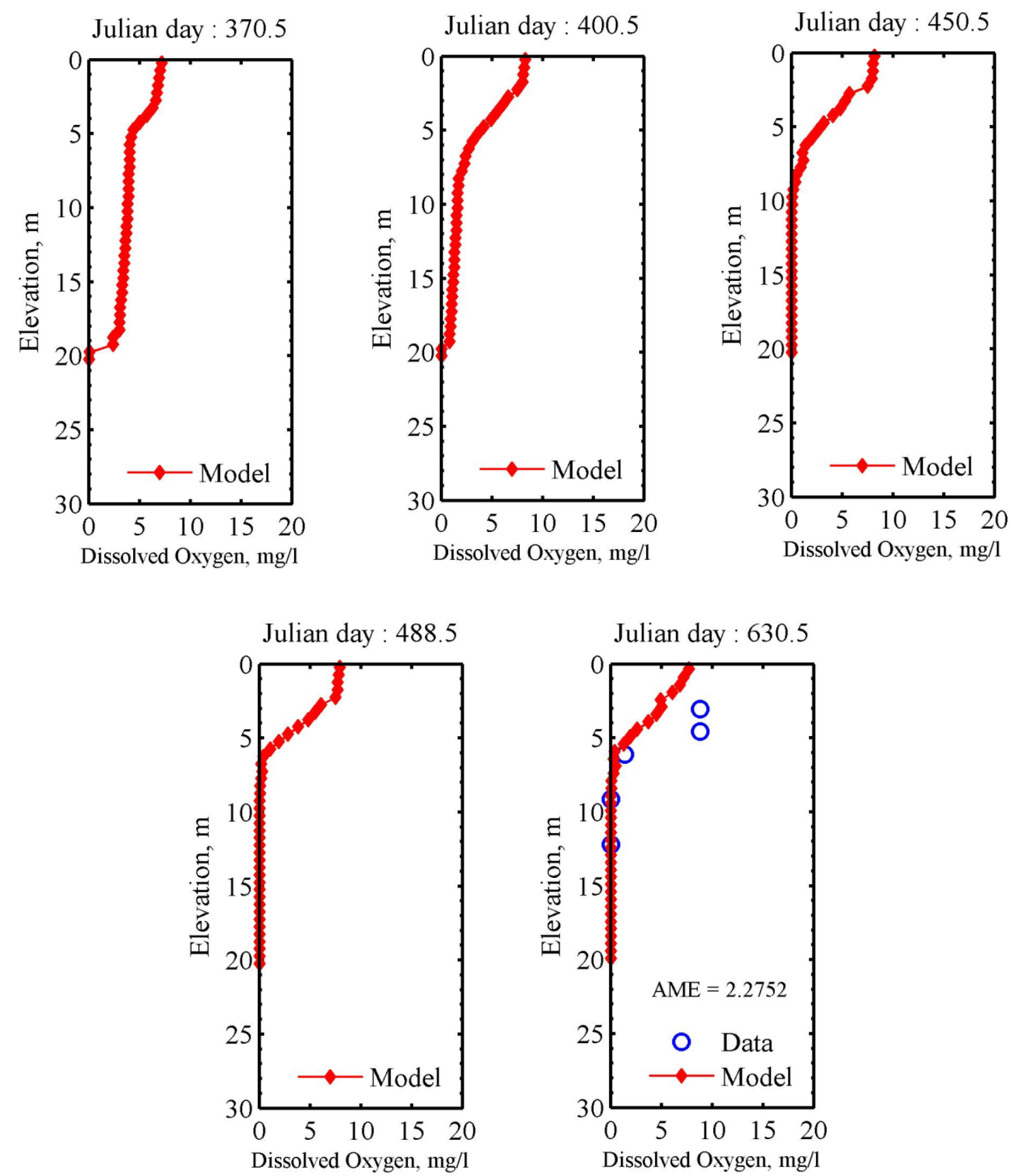

Figure 4-69. Cooper Creek Reservoir model predictions of vertical dissolved oxygen profiles compared with field data 
Table 4. Overall AME of CE-QUAL-W2 numerical predictions of Cooper Creek Reservoir model

\begin{tabular}{cccc}
\hline & Water Level, $\mathrm{m}$ & Temperature, ${ }^{\circ} \mathrm{C}$ & Dissolved Oxygen, $\mathrm{g} \mathrm{m}^{-3}$ \\
\hline \multirow{2}{*}{ AME } & 0.079 & 0.929 & 0.798 \\
& & & \\
\hline
\end{tabular}

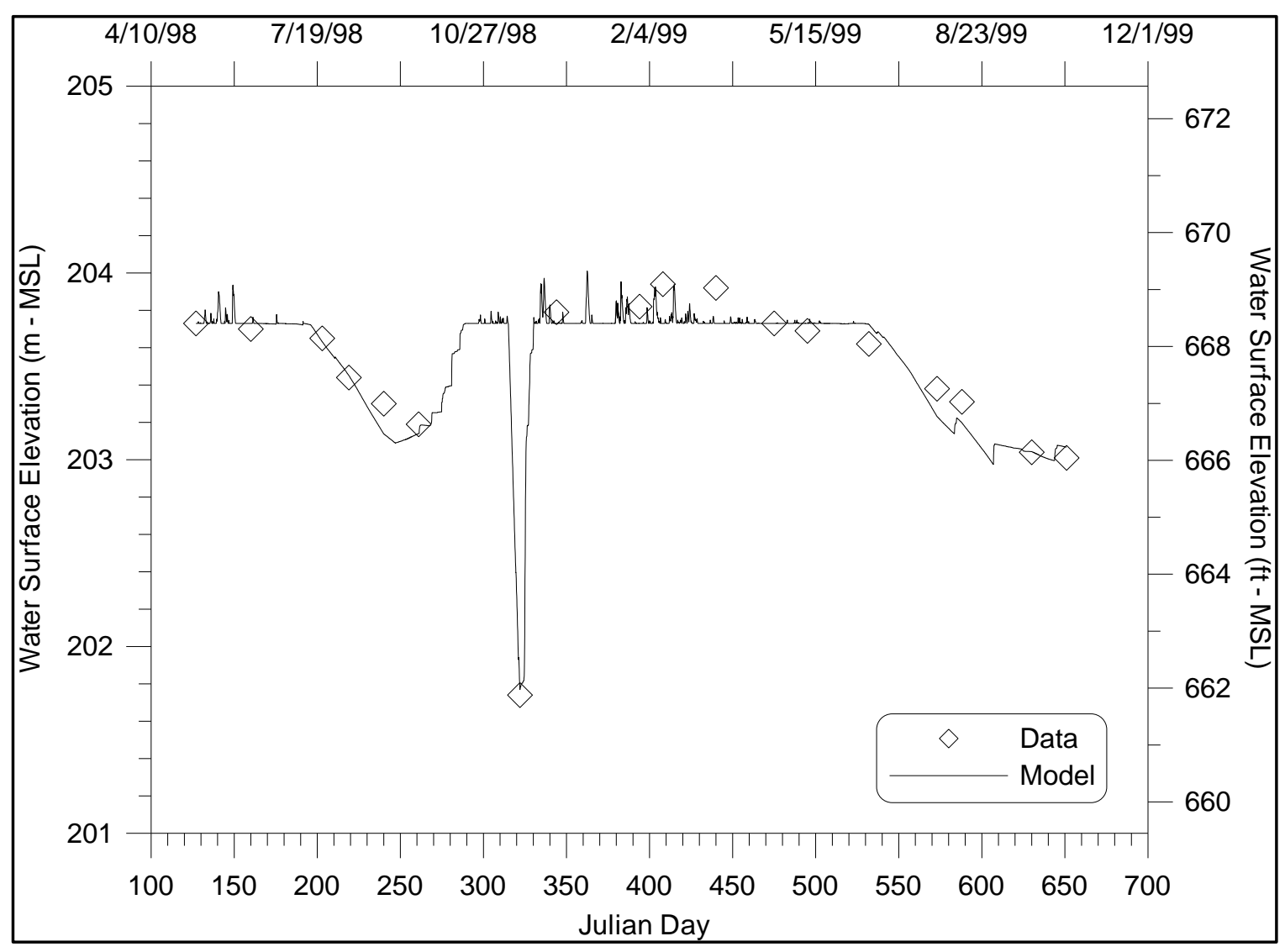

Figure 4-70. CE-QUAL-W2 water level numerical predictions of Cooper Creek Reservoir model compared to data 

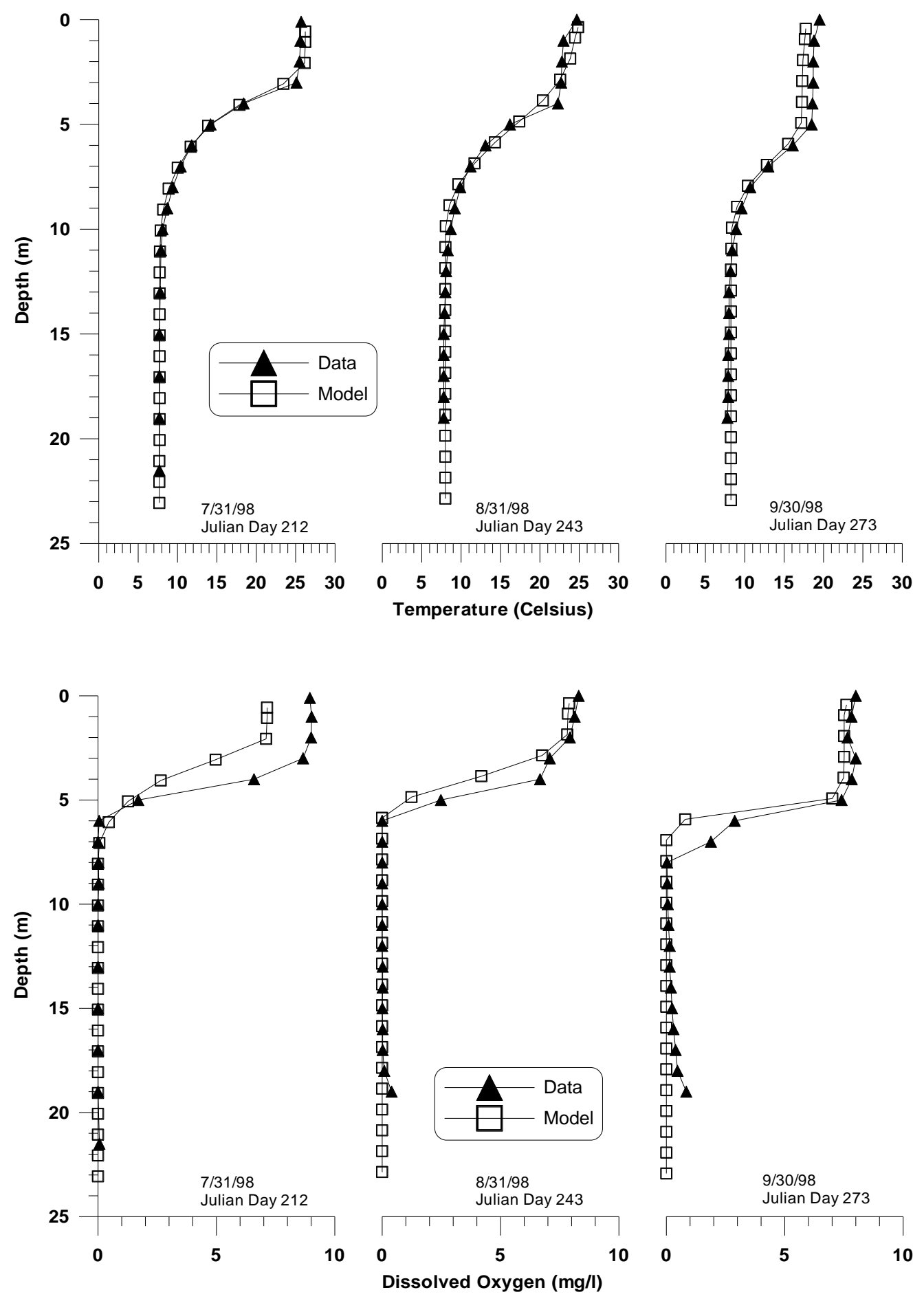

Figure 4-71. CE-QUAL-W2 vertical temperature and dissolved oxygen profiles of Cooper Creek Reservoir model compared to data 


\section{CHAPTER 5. CONCLUSIONS AND FUTURE STUDIES}

In this dissertation, a three-dimensional hydrodynamic, temperature, and water quality numerical model was developed, verified, and validated for surface waterbodies. The model was built based on many of the algorithms used in the 2D CE-QUAL-W2 model, a two-dimensional longitudinal/vertical hydrodynamic and water quality numerical model. CE-QUAL-W2 has been applied and used successfully to manage many waterbodies (rivers, lakes, reservoirs, and estuaries) around the world. In order to account for its advantages, the hydrodynamic numerical solution scheme was expanded and modified to a new three-dimensional scheme.

The model governing equations are the continuity equation, free surface equation, momentum equations, and conservation equations of temperature and water quality (Chapter 2). The model employs the $z$-coordinate system to remove the extra approximations and complexity associated with other coordinate transformation approaches such as the $\sigma$-coordinate. The model employs the time splitting technique to solve the momentum and transport equation numerically (Section 2.5.4 and 2.5.8). In this technique, the governing equation is split into two equations based on the horizontal and vertical transport of momentum and mass/heat, resulting in a numerical solution based on solving a tri-diagonal matrix form by the Thomas algorithm. Additionally, a new approach has been implemented in this model to deal with the penta-diagonal matrix resulting from the numerical solution of the free surface equation for a rectangular domain and a sparse matrix for a general domain. A method called line-by-line was applied (Section 2.5.9). The line-by-line method is a combination of direct and iterative 
numerical solution in which the sparse or penta-diagonal matrix form can be solved as a tri-diagonal matrix. Therefore, the final linear algebraic equations system generated from the free surface equation was solved by the Thomas algorithm.

The CE-QUAL-W2 hydrodynamic numerical scheme is fully implicit. It calculates the free surface elevation implicitly from the free surface equation. However, the numerical solution of the momentum equations solves the free surface elevation explicitly. In the present three-dimensional model, both numerical solutions (momentums and free surface) were linked in which the free surface elevation is treated either explicitly or implicitly at the same time step. This has been done by adding the degree of implicitness to the threedimensional numerical solution of free surface and momentum equations (Section 2.5.5). This employment improved the fully implicit scheme by reducing the free surface wave damping of the numerical solution (Section 3.1).

A novel approach compared to other three-dimensional models is that the threedimensional hydrodynamic numerical solution was coupled with the numerical solution of heat and water quality so that hydrodynamics, temperature, and water quality were solved at the same time step (Section 2.5.11 and 4.2.7). Most three-dimensional models involved with water quality modeling do not link water quality and hydrodynamics, hence there is no feedback between hydrodynamics and water quality processes affecting density such as algae growth and suspended solids through effects on light penetration (Section 4.2.7). 
Another new approach in this three dimensional model is that the model employs the 2D based selective withdrawal theory in the 3D case at the outlet structures of the dam and at any other location that requires removing water from a waterbody (Section 4.3.1). The selective withdrawal algorithm was already built in CE-QUAL-W2, providing a way to remove water not only from the water layer in front of the outlet but from multiple vertical layers based on the water density structure. The importance of using this algorithm at dam/reservoir withdrawals arises since it informs the model where to withdraw water from vertical layers without needing to solve the near-field dynamics with the vertical momentum equation.

The first step after building the numerical model was to verify that the model reproduced known analytical solutions. The verification of the model hydrodynamics was performed by comparing the model results of velocities and water levels with known exact solution test cases to show the numerical scheme behavior compared with the analytical solution. In addition, whether the code preserves fluid mass or not was evaluated by calculating the volume balance percent error over time during a model simulation. The following verification tests were performed (Chapter 3):

1. Test 1: Free surface seiching in a closed rectangular basin.

2. Test 2: Free water surface response to wind-induced flow in a closed rectangular basin.

3. Test 3: Velocity profile response to the wind induced flow in a closed rectangular basin.

4. Test 4: Volume balance: 
- Irregular physical domain.

- Rectangular physical domain.

5. Test 5: Model sensitivity to bottom friction.

6. Test 6: Model sensitivity to wind.

7. Test 7: Wetting/Drying boundary conditions.

In Tests 1 and 2, the model showed good agreement with the analytical solution for free surface seiching in a closed rectangular basin and in wind-induced seiching. Even though the model exhibited a stable solution, a lower time step showed a better match with the analytical solution. Thus, model stability does not guarantee model accuracy. In addition, it was found that implementing the semi-implicit scheme reduces the amount of free surface wave damping associated with the numerical scheme behaviour. Test 3 also showed good agreement between the velocity profile induced by wind in both the analytical and numerical 3D model. The model volume balance analysis in Test 4 was a test of the water volume in the model domain compared with the water volume entering and leaving the same domain during the same period of time. The volume balance error over time was computed. The model results indicated that the volume balance was better for regular grids and simple flows. Even though all the models had a reasonable volume balance error (less than $0.1 \%$ ), the semi-implicit numerical scheme had slightly better volume balance error than the fully-implicit scheme. In addition, the model sensitivity Tests 5 and 6 for bottom friction and wind shear stresses indicated that the model response to these was as expected by theory and that these are important factors in model calibration. Lastly, the wetting/drying boundary conditions were verified in Test 7 . 
Furthermore, applications were performed by modelling three reservoirs in the US as field case studies (Chapter 4): Lake Chaplain in WA, Laurance Lake in OR, and Cooper Creek Reservoir in OR. The case studies exercised the model capability to match field data of water surface elevations, vertical temperature, velocity, and dissolved oxygen. The comparisons between the model predictions and field data showed the model's ability to reproduce field data successfully. The results of the model applications were as follows:

1. In the Lake Chaplain model application (Section 4.1), the study was focused on the importance of the higher-order schemes compared to the first-order UPWIND scheme. The model predictions of temperature were determined by using the UPWIND, QUICK, and QUICKEST schemes and compared to field data. The model results indicated that higher-order schemes are much important for modeling temperature and water quality compared to the UPWIND scheme.

2. In the Laurance Lake model application (Section 4.2), an equivalent comparison was performed between the present three-dimensional model and the twodimensional CE-QUAL-W2 model. The QUICKEST and ULTIMATE QUICKEST schemes were applied for this system. The comparison results showed that the simulation time for the three-dimensional model was much longer than the two-dimensional CE-QUAL-W2 model (approximately 60 times longer). This computational time though for the 3D model was very dependent on the grid resolution. The grid development of the 2D model could be considered somewhat more difficult than for the 3D model since the 2D model setup required a dividing 
of the physical domain into branches of different flow paths and increments. The three-dimensional model though can more accurately assess lateral velocity variations if those are important to assess. The results also indicated that the twodimensional model predictions were somewhat more accurate than the $3 \mathrm{D}$ model. In addition, the total suspended solids were simulated to show the link between the hydrodynamics and water quality.

3. In the Cooper Creek Reservoir model application (Section 4.3), the study was done to show the model predictions of temperature and dissolved oxygen. Vertical temperature profiles covered the entire simulation period from pre-stratification to stratification to fall overturn. The model predictions were tested in the presence of two submerged withdrawals and an upper spillway flow at the dam location. The model predictions of the 3D model were in agreement with field data for water surface, dissolved oxygen and temperature.

Because this research focused on developing a three-dimensional model based on the numerical scheme of the 2D laterally averaged CE-QUAL-W2 model, we can summarize the main advantages and disadvantages of $2 \mathrm{D}$ compared to 3D models in Table based on our case studies (Chapter 4).

Therefore, choosing a 2D or 3D model depends mainly on the shape of the waterbody and the necessity to consider 3D effects, like a side discharge when mixing in the lateral dimension is an important aspect of management. The more round the waterbody, the more the velocity needs to be resolved in $3 \mathrm{D}$. The aspect ratio (length>width ratio) and the shape factor (shoreline length/circumference of an equivalent circular area) of the 
three waterbodies that we have simulated in this research were arranged as shown in Table 6.

Table 5. Advantages and disadvantages of using the 2D model compared to the 3D model

\begin{tabular}{|c|c|c|}
\hline Property & $2 \mathrm{D}$ & $3 \mathrm{D}$ \\
\hline Grid setup & - Harder & - Easier \\
\hline Computational time & - Lower & - Higher \\
\hline $\begin{array}{l}\text { Waterbody } \\
\text { (length/width) ratio }\end{array}$ & $\begin{array}{l}\text { length }>\text { width } \\
\text { (depending on the } \\
\text { domain regularity) } \\
\text { - length }>>\text { width }\end{array}$ & $\begin{array}{l}\text { - length }>\text { width } \\
\text { (depending on the } \\
\text { domain regularity) } \\
\text { - length =width } \\
\text { - } \quad \text { Shape factor }>=1.0\end{array}$ \\
\hline Lateral inflows & $\begin{array}{l}\text { Not as good as the } \\
\text { 3D model since } \\
\text { laterally averaged } \\
\text { and velocity is } \\
\text { averaged bank to } \\
\text { bank }\end{array}$ & $\begin{array}{l}\text { Better resolution of } \\
\text { travel time along } \\
\text { centerline and edges of } \\
\text { a lake or reservoir }\end{array}$ \\
\hline $\begin{array}{l}\text { Studies interested in } \\
\text { Pelagic or Lacustrine } \\
\text { water zone }\end{array}$ & $\begin{array}{l}\text { - Lacustrine and } \\
\text { Pelagic areas only in } \\
\text { shallower areas of a } \\
\text { lake or reservoir but } \\
\text { not along a cross- } \\
\text { section that includes } \\
\text { pelagic and } \\
\text { lacustrine waters }\end{array}$ & - Pelagic and lacustrine \\
\hline
\end{tabular}


Table 6. The shape factor corresponding to each case study

\begin{tabular}{lcccc}
\hline Waterbody & Length, $\mathrm{m}$ & Width, $\mathrm{m}$ & Aspect ratio & Shape factor \\
\hline $\begin{array}{l}\text { Cooper Creek } \\
\text { Reservoir }\end{array}$ & 2500 & 300 & 8.3 & 2.8 \\
Lake Chaplain & 3300 & 880 & 3.75 & 1.82 \\
& 1474 & 510 & 2.89 & 1.53 \\
\hline
\end{tabular}

As the aspect ratio of a lake or reservoir decreases, the shape factor decreases, and therefore as the waterbody shape approaches to the circular shape (shape factor of 1) or squared shape (aspect ratio of 1), the waterbody becomes more 3D. For instance, Cooper Creek Reservoir has a long shape compared to its very small width in addition to its high shape factor. To manage this by using the 3D model, the grid resolution needs to be high enough to cover the waterbody surface area. This leads to more computational time since the time step should be low for the model stability. Therefore, the Cooper Creek Reservoir case tends to be more 2D than Laurance Lake.

This research was also summarized in the following publications: Al-Zubaidi \& Wells (2018a), Al-Zubaidi \& Wells (2018b), Al-Zubaidi \& Wells (2018c), Al-Zubaidi \& Wells (2017d), Al-Zubaidi \& Wells (2017e). 
Whereas this three-dimensional model was developed, verified, and validated successfully by showing good agreement with the analytical solution and field data, the model could be further developed to allow more model capabilities. The following suggestions regarding the model development can improve the model capability:

1. Applying the model to other test cases to test the model resilience.

2. Applying the model to other kinds of surface waterbodies such as rivers, estuaries, and coastal areas. For estuaries, the model code needs more features added to represent tidal boundary conditions.

3. Adding other turbulent closure schemes to the present model to calculate the turbulent viscosity and diffusivity such as $\kappa-\varepsilon$ turbulent model as used now in CEQUAL-W2. Although other turbulent models could be more accurate in representing the turbulent transport of momentum, these models required extra complex programming since some of them need to solve additional threedimensional partial differential equations numerically.

4. Linking the present three-dimensional model with the two-dimensional CEQUAL-W2 model. This link could be during the simulation time by sharing the outputs, or by running one of the models and making the outputs as inputs to the other model.

5. Building a water balance tool for the present three-dimensional model to calculate the difference between the waterbody inflows and outflows. This tool already exists in CE-QUAL-W2 model. 
6. Adding other water quality constituents in addition to the sediment first-order algorithm to the present model in order to account for the interactions among the water quality constituents.

7. Re-developing the present model based on a non-equally spacing grid. Unequal grid spacing will affect the model stability since the model stability condition is a function of the grid resolution.

8. Developing a model-user interface for easing the development of model input files and boundary condition files 


\section{REFERENCES}

Ahsan, A. K. M., \& Blumberg, A. F. (1999). Three-dimensional hydrothermal model of Onondaga Lake, New York. Journal of Hydraulic Engineering, 125(9), 912-923. http://doi.org/10.1061/(ASCE)0733-9429(1999)125:9(912)

Al-Zubaidi, H. A. M., \& Wells, S. A. (2017a). 3D Numerical Temperature Model Development and Calibration for Lakes and Reservoirs: A Case Study. Proceedings, World Environmental and Water Resources Congress 2017, Sacramento, CA, USA. http://doi.org/10.1061/9780784480601.051

Al-Zubaidi, H. A. M., \& Wells, S. A. (2017b). 2D and 3D Numerical Modeling of Water Level and Temperature in Lakes and Reservoirs Based on the Numerical Scheme in CE- QUAL-W2: A Case Study. Poster session presented at the meeting of the 37th International Symposium of the North American Lakes Management Society, Westminster, Colorado, USA.

Al-Zubaidi, H. A. M., \& Wells, S. A. (2018c). Comparison of a 2D and 3D Hydrodynamic and Water Quality Model for Lake Systems. Proceedings, World Environmental and Water Resources Congress 2018, Minneapolis, Minnesota, USA. https://doi.org/10.1061/9780784481400.007

Al-Zubaidi, H. A. M., \& Wells, S. A. (2018d). Water Level, Temperature, and Water Quality Numerical Predictions of a 3D Semi-Implicit Scheme for Lakes and Reservoirs: An Analytical and Field Case Study. Proceedings of the 9th International Congress on Environmental Modelling and Software (iEMSs 2018), Ft. Collins, Colorado, USA. 
Al-Zubaidi, H. A. M., \& Wells, S. A. (2018e). Analytical and Field Verification of a 3D Hydrodynamic and Water Quality Numerical Scheme Based on the 2D Formulation in CE-QUAL-W2. Journal of Hydraulic Research, Taylor \& Francis. doi: 10.1080/00221686.2018.1499051

Annear, R. L., Berger, C. J., \& Wells, S. A. (2008). Lake Chaplain Model : Model Development, Calibration, and Management Scenarios. Technical Report EWR-0108 water quality research group, Department of civil and environmental engineering, Portland State University.

Annear, R. L., \& Wells, S. A. (2007). A Comparison of Five Models for Estimating Clear-Sky Solar Radiation. Water Resour., Res., 43(W10415). http://doi.org/:10.1029/2006WR005055.

Ater, K. B., \& Macdonald, P. (2013). Estuarine and Wetland Processes: With Emphasis on Modeling. Springer US.

Barron, C. N., Kara, A. B., Martin, P. J., Rhodes, R. C., \& Smedstad, L. F. (2006). Formulation, implementation and examination of vertical coordinate choices in the global Navy Coastal Ocean Model (NCOM). Ocean Modelling, 11, 347-375.

Batchelor, G. K. (1967). An Introduction to Fluid Dynamics. Cambridge University Press, NY.

Berger, C. J., Wells, S. A., \& Annear, R. L. (2005). Laurance Lake Temperature Model. Technical Report EWR-01-04 water quality research group, Department of civil and environmental engineering, Portland State University.

Blumberg, A. F., \& Mellor, G. L. (1980). A coastal ocean numerical model. Proc., Int. Symp. on Math. Modelling of Estuarine Phys., J. Sundermann and K. P. Holz, Eds., 242 
Springer, Berlin, 202-219.

Blumberg, A. F., \& Mellor, G. L. (1987). A description of a three-dimensional coastal ocean circulation model. Three-Dimensional Coastal Ocean Models. N. S. Heaps, Ed., American Geophysical Union, Washington, D.C., 1-16. http://doi.org/10.1029/CO004p0001

Bryan, K. (1969). A numerical method for the study of the circulation of the world ocean. Journal of Computational Physics, 4(3), 347-376. http://doi.org/10.1016/00219991(69)90004-7

Casulli, V., \& Cattani, E. (1994). Stability, accuracy and efficiency of a semi-implicit method for three-dimensional shallow water flow. Computers \& Mathematics with Applications, 27(4), 99-112. http://doi.org/10.1016/0898-1221(94)90059-0

Casulli, V., \& Cheng, R. T. (1992). Semi-implicit finite difference methods for threedimensional shallow water flow. International Journal for Numerical Methods in Fluids, 15(6), 629-648.

Casulli, V., \& Stelling, G. S. (1995). Simulation of three-dimensional, non-hydrostatic free-surface flows for estuaries and coastal seas. The 4th International Conference on Estuarine and Coastal Modeling, San Diego, USA, 1-12.

Casulli, V., \& Walters, R. (2000). An unstructured grid, three-dimensional model based on the shallow water equations. International Journal for Numerical Methods in Fluids, 32, 331-348. http://doi.org/10.1002/(SICI)10970363(20000215)32:3<331::AID-FLD941>3.0.CO;2-C

Chapra, S. C. (1997). Surface Water-Quality Modeling. Waveland Press, Inc. Chen, C., Beardsley, R. C., Cowles, G., Qi, J., Lai, Z., Gao, G., .. Lin, H. (2011). An 243 
Unstructured-Grid, Finite-Volume Community Ocean Model FVCOM User Manual (3rd Edition). Sea Grant College Program Massachusetts Institute of Technology Cambridge, Massachusetts 02139.

Chen, C., Liu, H., \& Beardsley, R. C. (2003). An unstructured grid, finite-volume, threedimensional, primitive equations ocean model: Application to coastal ocean and estuaries. Journal of Atmpspheric and Oceanic Technology, 20, 159-186.

Cole, T., \& Buchak, E. (1995). CE-QUAL-W2: A Two-Dimensional, Laterally Averaged, Hydrodynamic and Water Quality Model, Version 2.0 User Manual. Instruction Rep. EL-95-1, U.S. Army Corps of Engineers, Washington, D.C.

Cole, T., \& Wells, S. A. (2015). CE-QUAL-W2: A Two-Dimensional, Laterally Averaged, Hydrodynamic and Water Quality Model, Version 3.72. User Manual. Department of Civil and Environmental Engineering Portland State University Portland, OR.

Csanady, G. T. (2013). Circulation in the Coastal Ocean. Springer Netherlands.

Cushman-Roisin, B., \& Beckers, J. M. (2007). Introduction to Geophysical Fluid Dynamics: Physical and Numerical Aspects. Academic Press.

Dronkers, J. J. (1964). Tidal Computations in Rivers and Coastal Seas. North Holland Publishing Co., Amsterdam, The Netherlands.

Edinger, J. E. (2001). Waterbody Hydrodynamic and Water Quality Modeling : An Introductory Workbook and CD-ROM on Three-Dimensional Waterbody Modeling. American Society of Civil Engineers, Virginia, United state of America.

Edinger, J. E., Brady, D. K., \& Geyer, J. C. (1974). Heat exchange and transport in the environment. Report No. 14, Research Project RP-49. Electric Power Research 244 
Institution, Palo Alto, CA.

Eliason, D. E., \& Bourgeois, A. J. (1997). Validation of numerical shallow water models for stratified seiches. International Journal for Numerical Methods in Fluids, 24(8), 771-786. Retrieved from http://www.scopus.com/inward/record.url?eid=2-s2.00031126669\&partnerID=40\&md5=3a7aee5cb35d5d7576b253b699e7bc8d

EPA. (1971). Effect of Geographical Location on Cooling Pond Requirements and Performance. In Water Pollution Control Research Series, Report No. 16130 FDQ, 160 Pp. Retrieved from Water Quality Office, U.S. Environmental Protection Agency, Washington, D. C.

Fofonoff, N. P. (1962). Physical properties of seawater. In The Sea, Ideas and Observations on Progress in the Study of the Seas: Physical oceanography (pp. 330). Vol. 1, M. N. Hill, ed., Wiley Interscience, New York. Retrieved from https://books.google.com/books/about/The_Sea_Ideas_and_Observations_on_Progr e.html?id=P7xRAAAAMAAJ\&pgis $=1$

Ford, D. E., \& Johnson, M. C. (1983). An assessment of reservoir density currents and inflow processes. Technical Rpt. E-83-7, US Army Engineer Waterways Experiment Station, Vicksburg, MS.

Gill, A. E. (1982). Atmosphere-Ocean Dynamics. Academic Press, New York, NY.

Hamrick, J. M. (1992). A Three-Dimensional Environmental Fluid Dynamics Computer Code: Theoretical and Computational Aspects. The College of William and Mary, Virginia Institute of Marine Science. Special Report 317, 63 Pp.

Hansen, N. E. (1975). Entrainment in Two-Layered Flows. Institute of Hydrodynamics and Hydraulic Engineering,Technical Institute of Denmark, Series Paper No. 7. 
Helfrich, K. R., Adams, E. E., Godbey, A. L., Harleman, D. R. F., Helfrich, K. R., Adams, E. E., ... Harleman, D. R. F. (1982). Evaluation of Models for Predicting Evaporative Water Loss in Cooling Impoundments. Report CS-2325, Research Project 1260-17. Electric Power Research Institute, Palo Alto, CA, (March).

Hodges, B., \& Dallimore, C. (2006). Estuary, Lake and Coastal Ocean Model : ELCOM v2.2 Science Manual. Center for Water Research, University of Western Australia. Idso, S. B., \& Jackson, R. D. (1969). Thermal Radiation from the Atmosphere. Jornal of Geophysical Research, 74(23).

Kowalik, Z., \& Murty, T. S. (1993). Numerical Modeling of Ocean Dynamics. World Scientific.

Leendertse, J. J., \& Liu, S. K. (1975). A Three-Dimensional Model for Estuaries and Coastal seas: Vol. II, Aspects of Computation. The RAND Corporation, R-1764OWRT.

Leonard, B. P. (1979). A stable and accurate convective modelling procedure based on quadratic upstream interpolation. Computer Methods in Applied Mechanics and Engineering, 19, 59-98.

Leonard, B. P. (1991). The ULTIMATE conservative difference scheme applied to unsteady one-dimensional advection. Computer Methods in Applied Mechanics and Engineering, 88, 17-74.

Martin, P. J., Barron, C. N., Smedstad, L. F., Campbell, T. J., Wallcraft, A. J., Rhodes, R. C., ... Carroll, S. N. (2009). User's Manual for the Navy Coastal Ocean Model ( NCOM ). Ocean dynamic and prediction branch, Oceanography devision.

Martin, J.L., McCutcheon, S. C. (1999). Hydrodynamics and Transport for Water Quality 246 
Modeling. CRC Press, Boca Raton, FL.

Mellor, G. L. (2002). Users guide for a three-dimensional, primitive equation, numerical ocean model (June 2003 version). Prog. in Atmos. and Ocean. Sci, Princeton University.

Mellor, G. L., \& Yamada, T. (1982). Development of a turbulence closure model for geophysical fluid problems. Reviews of Geophysics, 20(4), 851. http://doi.org/10.1029/RG020i004p00851

Millero, F. J., \& Poisson, A. (1981). International one-atmosphere equation of state of seawater. Deep Sea Research, 28A, 625-629.

Mortimer, C. H. (1981). The Oxygen Content of Air Saturated Fresh Waters over Ranges of Temperature and Atmospheric Pressure of Limnological Interest. International Vereinigung Theo-Retische and Angewandte Limnologie, 22, 2-23.

Neumann, L. E., Simunek, J., \& Cook, F. J. (2011). Implementation of quadratic upstream interpolation schemes for solute transport into HYDRUS-1D. Environmental Modelling \& Software, 26, 1298-1308.

Okubo, A. (1971). Oceanic diffusion diagrams. Deep-Sea Research, 18(8), 789-802.

Patankar, S. V. (1980). Numerical Heat Transfer and Fluid Flow. Hemisphere Publishing Corporation, McGraw Hill Book Co., New York.

Press, W. H., Teukolsky, S. A., Vetterling, W. T., and Flannery, B. P. (1992). Numerical Recipes in Fortran: The Art of Scientiffic Computing. 2nd ed. Cambridge University Press, Cambridge

Rodi, W. (1980). Turbulence Models and Their Application in Hydraulics - a State of the Art Review. Association Internationale Des Recherches Hydrauliques. 
Ryan, P. J., \& Harleman, D. R. F. (1973). An Analytical and Experimental Study of Transient Cooling Pond Behavior. Report No. 161. Ralph M. Parsons Laboratory,. Department of Civil Engineering, Massachusetts Institute of Technology, Cambridge, MA.

Shanahan, P. (1985). WATER TEMPERATURE MODELING: A PRACTICAL GUIDE. Environmental Research and Technology, Inc. Concord, Massachusetts, (January). Sheng, Y., \& Butler, H. (1982). Modeling Coastal Currents and Sediment Transport. Coastal Engineering, 1127-1148. http://doi.org/10.1061/9780872623736.070

Smagorinsky, J. (1963). General circulation experiments wiht the primitive equations I. The basic experiment. Monthly Weather Review, 91(3), 99-164. http://doi.org/10.1126/science.27.693.594

Smith, D. J. (1978). WQRRS, Generalized computer program for River-Reservoir systems. USACE Hydrologic Engineering Center HEC, Davis, California. Smith, P. E. (2006). A Semi-Implicit, Three-Dimensional Model for Estuarine Circulation. U.S. Geological Survey Open-File Report 2006-1004, 176 p.

Swinbank, W. C. (1963). Long-Wave Radiation from Clear Skies. Quarterly Journal of the Royal Meteorological Socity of London, 89, 339-348.

Tetra Tech, I. (2002). Draft user's manual for environmental fluid dynamic code Hydro Version (EFDC-Hydro) Release 1.00. Tetra Tech, Inc., Fairfax, Virginia. Tetra Tech, Inc., Fairfax, Virginia.

Thomann, R. V., \& Mueller, J. A. (1987). Principles of surface Water Quality Modeling and Control. Harper \& Row.

Thornton, K. W., \& Lessem, A. S. (1978). A temperature algorithm for modifying 248 
biological rates. Transactions of the American Fishery Society, 107, 284-287.

UNESCO. (1981). Tenth report of the Joint Panel on Oceanographic Tables and

Standards. Technical Papers in Marine Science, No. 36, Sidney, B.C., Canada.

Vreugdenhil, C. B. (1989). Computational Hydraulics An Introduction. Springer-Verlag

Berlin Heidelberg. Springer-Verlag Berlin Heidelberg. http://doi.org/10.1007/978-3$642-95578-5$

Wang, H., \& Falconer, R. A. (1998). Numerical modeling of flow in chlorine disinfection tanks. Journal of Hydraulic Engineering, 124(9), 918-931.

Wang, S. Y., Roache, P. J., Schmalz, R. A., Jia, Y., \& Smith, P. E. (2009). Verification and Validation of 3D Free-Surface Flow Models. American Society of Civil Engineers, United State of America.

Wells, S. A. (2002a). Basis for the CE-QUAL-W2 version 3 river basin hydrodynamic and water quality model. ASCE International Water Resources Engineering Conference, $1-11$.

Wells, S. A. (2002b). Validation of the CE-QUAL-W2 Version 3 River Basin Hydrodynamic and Water Quality Model. Proceedings, HydroInformatics 2002, IAHR, Cardiff, England.

Wells, S. A., Annear, R. L., Berger, C. J., \& Sytsma, M. (2000). Modeling and Analysis of Cooper Creek Reservoir Water Quality. Technical Report, Water quality research group, Department of civil and environmental engineering, Portland State University, prepared for the City of Sutherlin, Oregon.

Wells, S. A., Curtis, D. C., Adams, E. E., Rodriguez, J. A., Eagleson, P. S., Bras, R. L., ... Harleman, D. R. F. (1982). Calibration and Verification of the Cooling Lake 249 
Model for North Anna Power Station. Technical Report 272, R.M. Parsons Laboratory, Department of Civil Engineering, School of Engineering, Massachusetts Institute of Technology.

Wu, J. (1969). Wind stress and surface roughness at air-sea interface. Journal of Geophysical Research, 74(2), 444-455. http://doi.org/10.1029/JB074i002p00444 Wunderlich, W. O. (1972). Heat and Mass Transfer Between a Water Surface and the Atmosphere. Water Resources Research Laboratory Report No. 14. (TVA)Tennessee Valley Authority, Division of Water Control Planning, Engineering Laboratory, Norris, TN. 